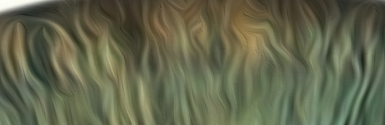
(4) 150 (a)

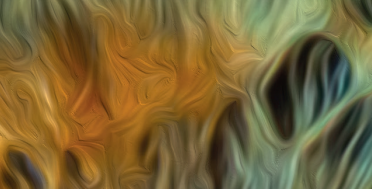

ary ande
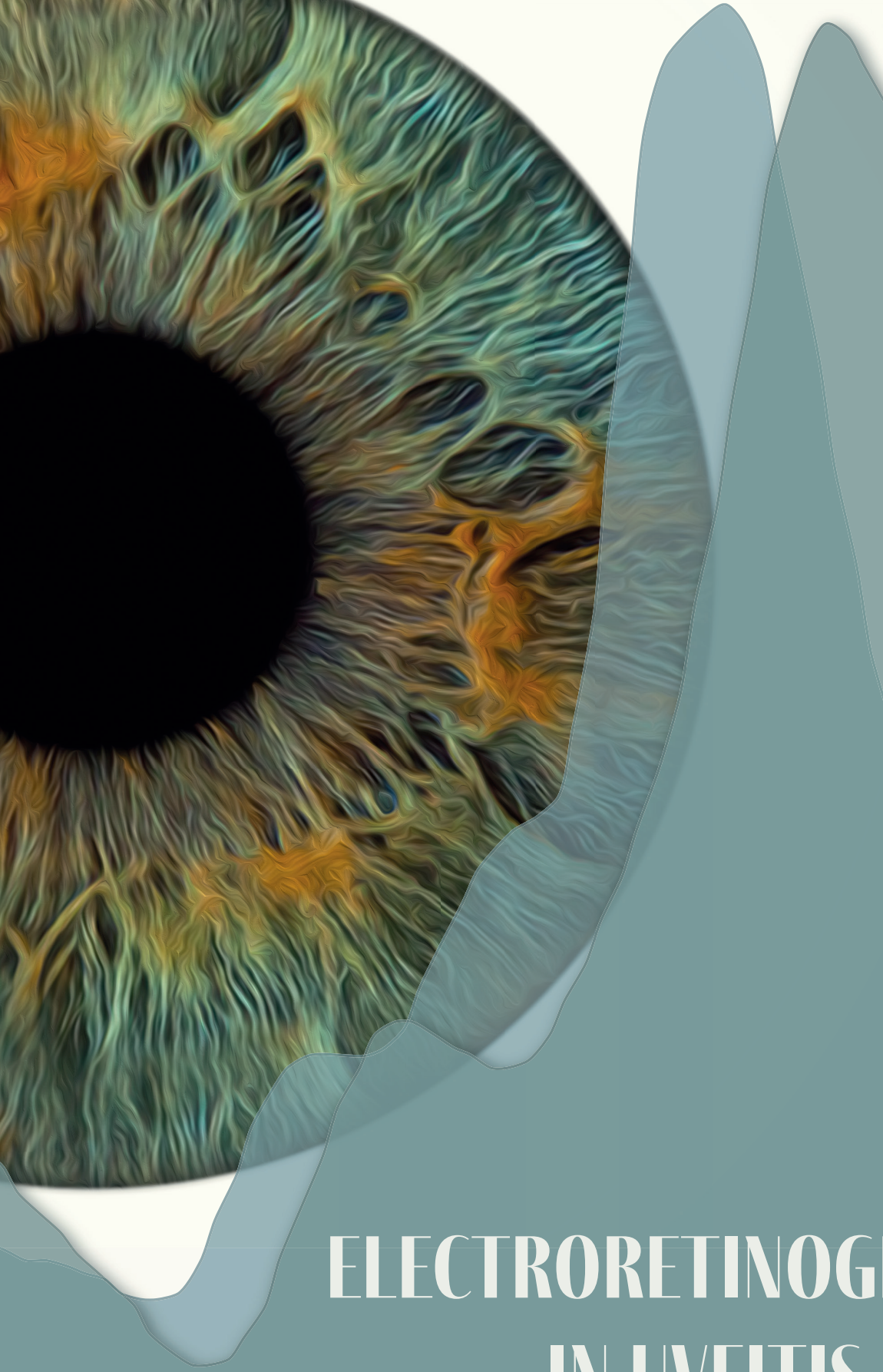

FIECTROREIINOGRAPHIY

IN UVEITIS 



\section{ELECTRORETINOGRAPHY IN UVEITIS}

Anna Brouwer 
Cover and lay-out: $\quad$ A.H. Brouwer and P.C. Kooijman

Printed by:

Ridderprint

ISBN:

978-90-393-7346-0

(c) 2020 Anna Brouwer

All rights reserved. No part of this thesis may be reproduced, distributed, or transmitted in any form or by any means, without the prior permission of the author. The copyright of the articles that have been published have been transferred to the respective journals.

The printing of this thesis was kindly supported by: Rotterdamse Stichting Blindenbelangen, Stichting Blindenhulp, Landelijke Stichting voor Blinden en Slechtzienden, Théa Farma, Bayer, Horus Pharma, Tramedico, Chipsoft, and Infection \& Immunity Utrecht.

The research in this thesis was financially supported by the Dr. F.P. Fischer Stichting and Bartiméus Fonds. 


\section{ELECTRORETINOGRAPHY IN UVEITIS}

\section{Electroretinografie bij uveitis}

(met een samenvatting in het Nederlands)

\section{PROEFSCHRIFT}

ter verkrijging van de graad van doctor aan de

Universiteit Utrecht

op gezag van de

rector magnificus, prof.dr. H.R.B.M. Kummeling,

ingevolge het besluit van het college voor promoties

in het openbaar te verdedigen op

dinsdag 10 november 2020 des middags te 12.45 uur

door

Anna Henriette Brouwer

geboren op 9 februari 1991

te Amsterdam 
Promotoren

Copromotor prof. dr. J.H. de Boer

prof. dr. M.M. van Genderen

dr. ir. G.C. de Wit 
Voor

Wiebe en Robijn 
Beoordelingscommissie Prof. dr. B. Oldenburg

Prof. dr. A. Rothova

Prof. dr. R.L.A.W. Bleys

Prof. dr. N.M. Jansonius

dr. M.J. van Schooneveld

Paranimfen

Pieter Kooijman

Sara Risseeuw 


\section{TABLE OF CONTENTS}

CHAPIER 1 General introduction, aims and scope of this thesis

CHAPIER 2 Prolonged cone b-wave on electroretinography is associated with severity of inflammation in noninfectious uveitis

Am J Ophthalmol. 2019, doi:10.1016/j.ajo.2019.05.028

CHAPIER 3 Electroretinogram abnormalities in non-infectious uveitis often persist

Acta Ophthalmol. 2020, doi: 10.1111/aos.14401

CHAPIER 4 Prolonged cone b-wave implicit time on electroretinography in relation to retinal layer analysis Manuscript submitted

CHAPIER 5 Electroretinogram abnormalities in non-anterior childhood uveitis

Acta Ophthalmol. 2019, doi: 10.1111/aos.13923

CHAPIER 6 Juvenile idiopathic arthritis uveitis: a typical anterior uveitis, but the retinal function can be affected

Manuscript submitted

CHAPIER 7 Effects of DTL electrode position on the amplitude and implicit time of the electroretinogram

Doc Ophthalmol. 2020, doi:10.1007/s10633-019-09733-3

CHAPIER 8 Summary, discussion \& future perspectives

CHAPIER 9 Nederlandse Samenvatting 


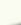

ambires) (b) ) 58 M(c) $y=10$ a) 9 mispere Qun (i)
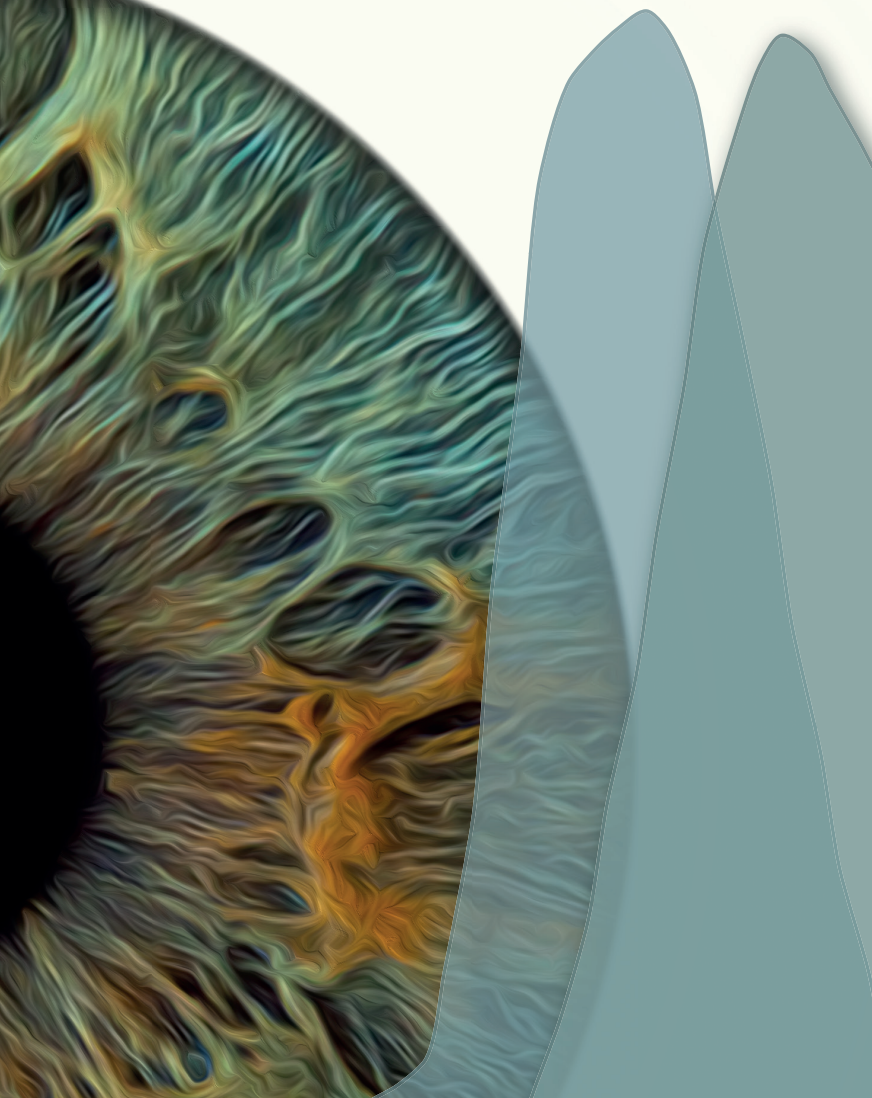
(9) (3)

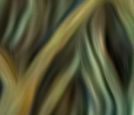

a mamme

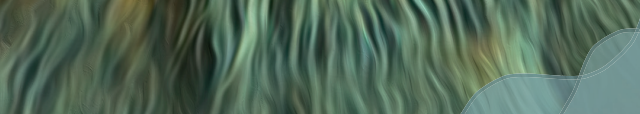

(19)

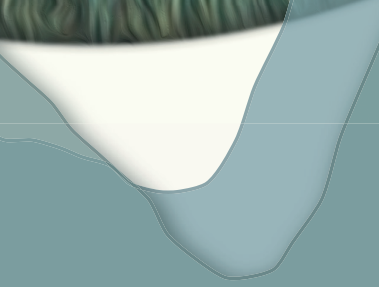




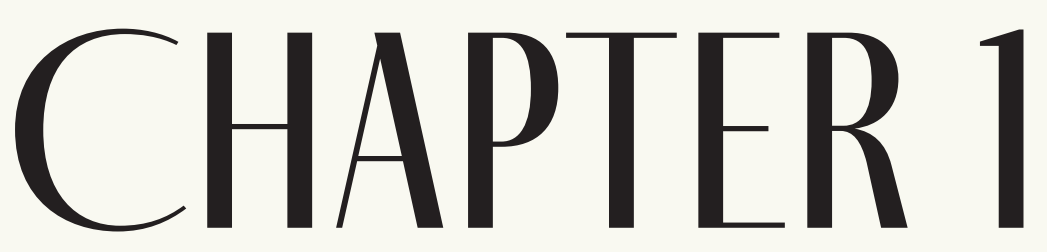

GENERAL INTRODUCTION, AIMS AND SCOPE OF THIS THESIS 


\section{INTRODUCTION}

Both anatomy, which describes the structure of organisms and their parts, and histology, which describes the structure of tissues, have helped scientists and doctors tremendously in their understanding of diseases. This understanding has led to new treatments and better prognoses for many patients. However, anatomy and histology do not always correspond with function.

The development and improvement of imaging techniques such as $\mathrm{X}$-rays, magnetic resonance imaging (MRI), and optical coherence tomography (OCT) have improved the diagnostic capacities of doctors even more, because they help to visualize structures in a non-invasive, and 3D manner.

But, even though structures can tell us a lot about a tissue, function is often far more important for patients in their daily functioning. In ophthalmology, patients can surprise their physicians with a great visual function, despite a very abnormal scan. Unfortunately, the opposite may also be true. This thesis focusses on one aspect of function: the electrophysiological function of the retina in a disease called uveitis.

\section{$\underline{\text { UVEITIS }}$}

\section{Anatomy and anatomical classification of uveitis}

When physiologists started to examine the eye, the ocular vascular bed appeared to them to have a some-what grape-like shape, after the sclera had been peeled off. They called it uvea: the Latin word for grape. The anatomical structure that we call the uvea nowadays, comprises the choroid, the ciliary body, the pars plana, and the iris. An inflammation of the uvea is called a uveitis. Interestingly, when ophthalmologists use the term uveitis, not only an inflammation of the uvea is implied; the adjacent structures, such as the retina, vitreous and anterior chamber may also show signs of inflammation.?

When ophthalmologists describe and classify uveitis, they try to determine the predominant site of the inflammation. This may be localized in the front, the middle, or in the back of the eye. Based on this anatomical localization, uveitis is called an anterior, intermediate, or a posterior uveitis. Sometimes no predominant site of inflammation can be defined: the entire eye appears to be inflamed. In such cases ophthalmologists call it a panuveitis.' Figure 1 illustrates this anatomical classification of uveitis. 


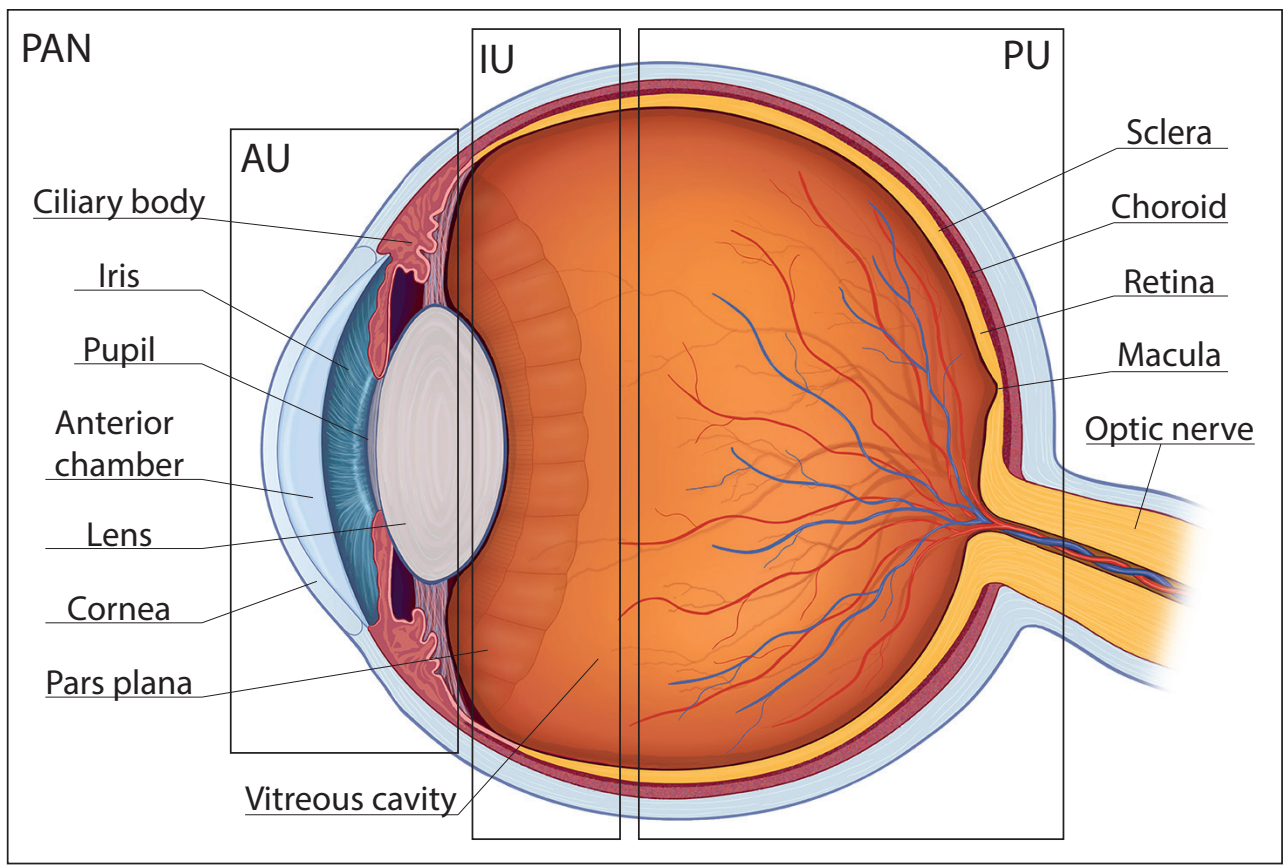

Figure 1: Illustration of the anatomy of the eye and it's relation to the different anatomical localizations of uveitis. Illustration recreated from: https://vsp.postclickmarketing.com/EyeAnatomyQuiz/eye-anatomyreview-dynamic-200WM-3388L1.html Abbreviations: $A U=$ anterior uveitis, $U I=$ intermediate uveitis, $P U=$ posterior uveitis, PAN = panuveitis.

\section{Causes of uveitis and associations with systemic diseases}

The term 'uveitis' comprises about 30 disease entities. Each of these individual diagnoses have certain typical characteristics, but there are also overlapping aspects. This makes it sometimes quite difficult to distinguish the different uveitis diagnoses from one-another.

Uveitis may be caused by infectious agents, which may be identified by serology, or analyzing ocular fluids, or both. ${ }^{2-4}$ However, many forms of uveitis are non-infectious. These non-infectious cases are often assumed to be auto-immune. However, in only about $5 \%$ of non-infectious uveitis cases, specific auto-antibodies can be found. ${ }^{5}$ Many forms of uveitis have an association with systemic diseases including sarcoidosis, Bechterew, psoriasis, Behçet's disease, multiple sclerosis (MS) and juvenile idiopathic arthritis (JIA). ${ }^{6-11}$ Sometimes the systemic disease is present prior to the uveitis, but in other cases the uveitis may be the first sign of the underlying systemic disease.

Uveitis also has several associations with certain human leukocyte antigen (HLA) types, including HLA-A29 (birdshot uveitis), 12,13 HLA-B27 (HLA-B27 associated 
uveitis), 10,14 and HLA-B51 (Behçet's disease). ${ }^{15}$ Lastly, there are several diseases which can mimic symptoms of uveitis, known as masquerade syndromes. ${ }^{16-18}$

One of the diseases that can be mistaken for uveitis are retinal dystrophies $(R D)$, a group of genetic diseases that are characterized by progressive rod and cone dysfunction and degeneration. Unfortunately there is currently no treatment for RD. Therefore it is important to distinguish RD from uveitis, because uveitis can be be treated with immunomodulating medication to prevent retinal damage. This treatment is not useful in RD, so patients should not be exposed to the possible harmfull sideeffects. ${ }^{18}$

Despite all the known causes and associations for uveitis, often no underlying cause for the inflammation can be found. 19,20 This thesis focusses on the electrophysiology of the retina in non-infectious uveitis, and includes all anatomical localizations of uveitis as well as all the underlying causes.

\section{Epidemiology and treatment of uveitis}

The prevalence of each of the different uveitis diagnoses is quite rare. However, as a group, uveitis is a much more common disease. The prevalence of uveitis varies around 85-204 cases per 100.000 cases, and the incidence is around 17-52 cases per 100.000 person-years. ${ }^{21-23}$ The peak incidence of uveitis is around 25-44 years of age, but some forms of uveitis present typically in childhood, whereas others manifest later in life. Because uveitis affects many people in the working population, the visual impact is quite high: uveitis causes about $10-15 \%$ of the total blindness in the United States. ${ }^{24-26}$

Treatment of uveitis depends on the severity and underlying cause. In case of infectious uveitis, the underlying pathogen needs to be tackled. In case of noninfectious uveitis, the treatment is often focused on reducing the inflammation to prevent permanent damage to ocular tissue. Depending on the severity, localization and laterality of the inflammation, treatment can start with steroid eye droplets, streroid injections, or systemic medication. . $^{20,27}$

Systemic steroids are usually not administered for a long time, because the longterm treatment has negative side-effects. Therefore, if an ophthalmologist expects that long-term treatment is necessary, a disease-modifying antirheumatic drug (DMARD) (e.g. methotrexate, azathioprine, mycophenolate mofetil, mycophenolate sodium, cyclosporine, cyclophosphamide) or a biologic (ladalimumab or infliximab) is usually commenced as well. 20,28 


\section{Complications of uveitis}

Many complications can occur due to uveitis. Depending on the specific cause of uveitis, the incidence of these complications varies. Complications that can occur on the anterior segment of the eye include: cataracts, band keratopathy, corneal decompensation, ciliary body dysfunction, posterior synechiae and a pupillary occlusion. . $^{2930}$

Complications to the posterior segment include cystoid macula edema (CME), glaucoma, vasculitis, retinal detachments, subretinal fibrosis, retinal neovascularization, retinal scarring, retinal atrophy and sometimes even phthisis bulbi. ${ }^{29-32}$

Several retinal changes can occur as well, which may result in retinal dystrophy like changes such as thinning of the retina, macula edema, and thinned vessels. ${ }^{33-35}$ These changes can make it quite difficult to distinguish uveitis from retinal dystrophies. ${ }^{18}$ But because uveitis can also lead to retinal atrophy, it would be useful to find early signs of retinal damage at a stage when it is still reversible..$^{32,36}$

\section{Monitoring uveitis activity and severity}

Uveitis is monitored using multiple tests and examinations. What exact work-up is done, depends on the type and severity of uveitis. Generally it includes a best corrected visual acuity (BCVA), slit-lamp examination and funduscopy. ${ }^{20}$

The amount of inflammatory cells in both the anterior chamber and vitreous are graded according to the Standardisation of Uveitis Nomenclature (SUN) criteria. The amount of leakage of proteins due to the inflammation is graded in the anterior chamber as flare, and in the vitreous as vitreous haze.'

Ancillary tests that use imaging include optical coherence tomography (OCT), fluorescein angiography (FA) and indocyanine green angiography (ICG). The OCT is used to assess the structure of the retinal layers. It uses differences in interference between tissues to create images with a much higher resolution than ultrasound: OCT scans show the structure of the retinal layers in a manner which resembles histology. Figure 2 gives an overview of the different retinal layers which can be identified on OCT and their relation to histology.

Invasive imaging techniques that are frequently used include FA and ICG to identify vascular leakage or vascular occlusions. FA is used to evaluate the retinal vasculature, whereas ICG is used to visualize pathology in the choroid, the vascular layer beneath the retina. ${ }^{37}$

A frequently used physiological test is visual field analysis (VF). It is used to monitor and diagnose glaucoma, a frequent complication of uveitis, neuro-ophthalmologic disorders, but it can also be used to monitor progressive visual field loss in birdshot uveitis. Lastly, functional tests that are sometimes used are the electroretinogram (ERG) and visually evoked potentials (VEP). 


\section{ELECTRORETINOGRAM}

The electroretinogram (ERG) is an objective test which assess the function of the retina. It measures the electrical signal of the cells of the retina in a response to light. In ophthalmology the ERG was originally used to help diagnose rare retinal disorders that affect retinal function. However, it is also sometimes used to monitor the progression of disease and to assess the effect of treatment.

\section{Types of electroretinograms}

Over the years several types of ERGs have been developed: the full-field ERG (ffERG) also known as Ganzfield ERG, the pattern ERG (PERG), and the multifocal ERG (mfERG). ${ }^{38-40}$ The full-field ERG measures the response of nearly the entire retina in reaction to a flash of light. This flash of light is emitted from a so-called Ganzfield dome, which ensures an even illumination of the retina.

Another type of ERG, the PERG, is said to reflect the macular and ganglion cell function. ${ }^{40}$ The mfERG is used to measure the function of different regions of the

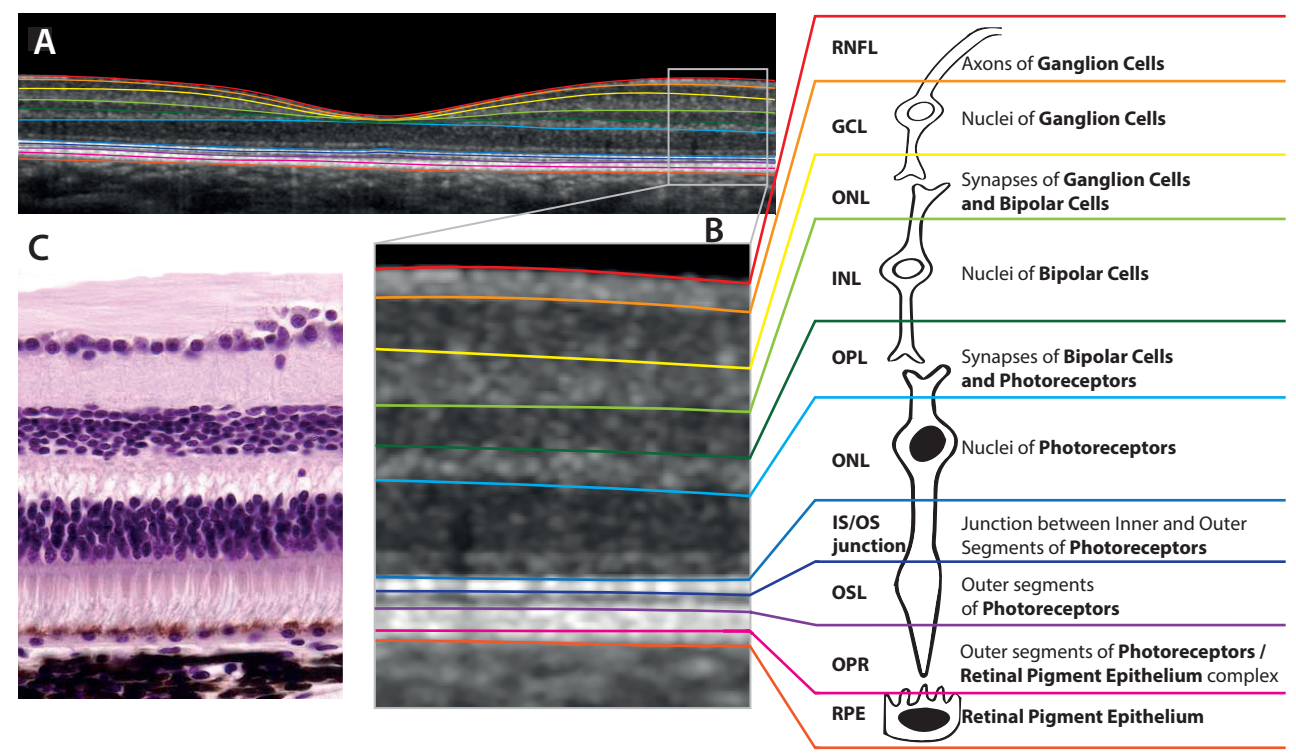

Figure 2: Optical coherence tomography scan of the retina in relation to retinal anatomy. A) OCT scan of the macula with segmentation lines indicating the boarders of several retinal layers. B) An enhancement of a part of OCT scan A. On the right of this enhancement is a scematic explanation of the different retinal cells of the segmented retinal layers. C) Histology biopt of a (different!) retina. Abbreviations: RNFL = Retinal Nerve Fibre Layer, $G C L$ = ganglion cell layer, IPL = inner plexiform layer, INL = inner nuclear layer, $O P L=$ outer plexiform layer, $O N L=$ outer plexiform layer, IS/OS junction = inner segment outer segment junction, OLS = outer segment layer, $O P R=$ outer segment photoreceptors/ retinal pigment epithelium complex, RPE = retinal pigment epithelium. 
central retina, including the macula. ${ }^{39}$ This thesis focusses on the full-field ERG, which is the most commonly used technique. For the rest of this thesis, when ERG is written, the full-field ERG is implied.

\section{ERG waves and types of adaptations}

There are several waves on an ERG curve that can be identified (Figure 2). The first is the negative a-wave. The a-wave represents the depolarization of the photoreceptors after a flash of light has been emitted.

The second wave is the (positive) b-wave. This wave is generated by the bipolar and Müller cells. On the rim of the ascending limb of the b-wave a 'saw-tooth' pattern can be observed. These are known as the oscillary potentials (OPs). The origin of these OPs remains unclear, some reports state that they reflect the amacrine cells, whereas others state that they are the result of bipolar cell activity. ${ }^{41}$

Besides the a-wave and b-wave, the c-wave and $d$-wave can be recorded as well. These represent the retinal pigment, and the OFF-center bipolar cells respectively. However, to record these waves, a much longer recording time is required. ${ }^{42,43}$ Therefore, they are not routinely recorded and are not a subject of this thesis.

Lastly, a more recent aspect of the ERG was identified: the photonegative response (PhnR). ${ }^{44}$ The PhnR can be observed after the b-wave. It is the negative reflection of the ERG curve which occurs after the positive b-wave. The PhnR reflects ganglion cell function and may be useful for detecting progression of glaucoma. ${ }^{45}$ The PhnR is not a subject of this thesis, because its identification is not a part of our local ERG protocol.

When the eyes are adapted to light, the ERG response is primariliy cone driven (photopic response). When the eyes are adapted to the dark, the response to dim (weak) flashes is rod driven (scotopic response). As the flashs strengths become stronger (i.e the light flashes become brighter), a combined rod/cone response is recorded (mesopic response).

\section{ISCEV standard and extended ISCEV protocols}

To ensure that the results of electrophysiological research of vision would be better comparable, the International Society for Clinical Electrophysiology of Vision (ISCEV), has created multiple guidelines to record ERGs, as well as other electrophysiological tests. ${ }^{38-40,46-48}$ Nowadays, virtually all scientific papers that report ERGs adhere to these standards.

Regarding the ERG, the ISCEV standard states the requirements for the equipment, flash strengths, background illumination, and the adaptation times for both 


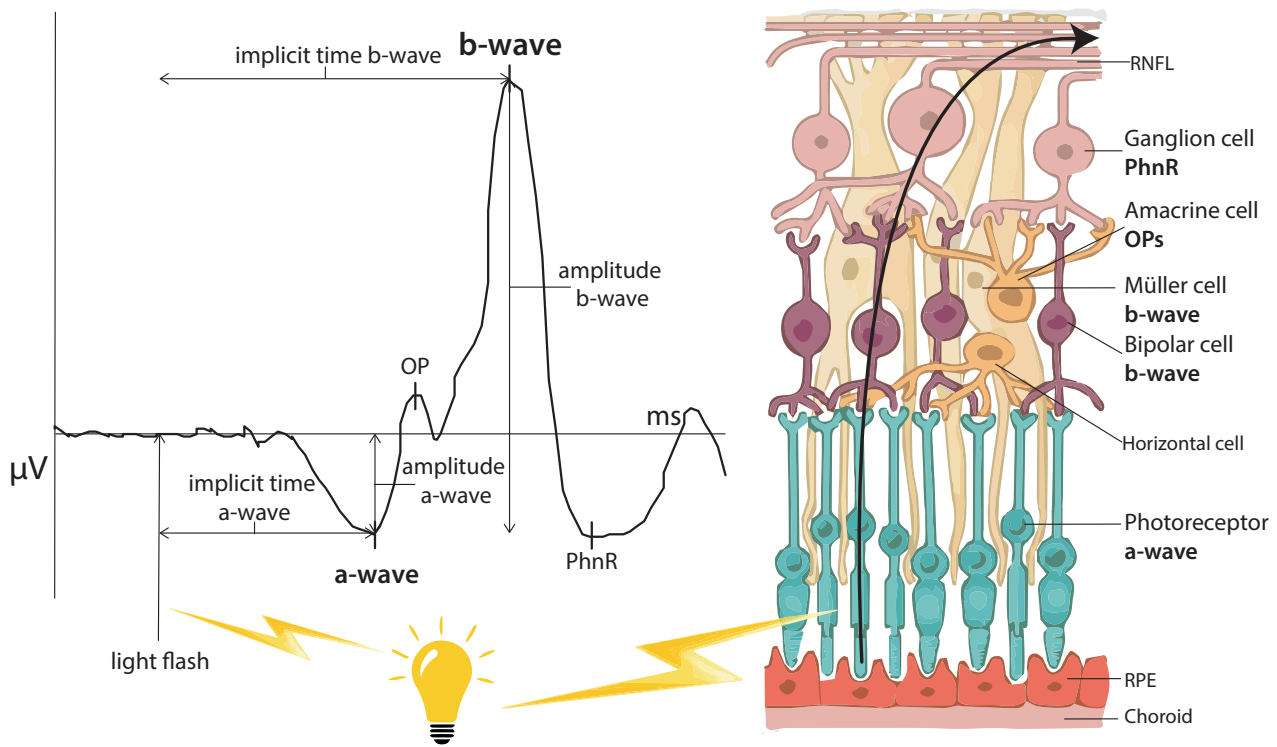

Figure 3: Explanation of ERG curves in relation to retinal cells. Abbreviations: $E R G=$ electroretinogram, $\mathrm{ms}=$ miliseconds, $\mu V=$ microvolt,$O P=$ ocillary potential, $P h n R=$ photonegative response, $R N F L=$ retinal nerve fibre layer, RPE = retinal pigment epithelium.

dark adaptation (DA) and light adaptation (LA). The guidelines are updated regularly and with each update the writers try to ensure that this is done in such a manner that research can still be compared, even when a previous ISCEV version was adhered to. This implies that flash strengths are added, but not removed. ${ }^{49}$

Besides the 'basic' ISCEV standards, which state the minimum requirements for an ERG (DA 0.01 (rod response), DA 3.0 and $10.0 \mathrm{cds} / \mathrm{m}^{2}$ (combined rod/cone response), LA $3.0 \mathrm{cds} / \mathrm{m}^{2}$ (cone response) and $30 \mathrm{~Hz}$ (cone response)), ISCEV has also created multiple extended protocols. These extended protocols are mostly used in a scientific setting. However, in several Dutch hospitals, including the University Medical Center Utrecht (UMCU) and Bartiméus diagnostic Centre for complex visual disorders in Zeist, an extended protocol is recorded as standard practice. Recently new extended protocols were published. Many of the flash strengths of the extended protocol that was used in this thesis overlap with these new extended protocols, but not all. ${ }^{50,51}$ Figure 4 shows an example of ERG curves of a healthy control of the extended protocol that was used in this thesis.

The ISCEV standards also describe how reference values should be obtained. It is important to have local reference values, because the type of equipment, type of electrodes, and local protocols can all influence the recorded ERG responses. Regarding amplitudes, the ISCEV standard states that amplitudes below the $5^{\text {th }}$ percentile should be considered abnormal. Implicit times are considered abnormal 
if they are above the $95^{\text {th }}$ percentile. These one-sides values are probably chosen because most diseases that affect the ERG result in either reduced amplitudes or increased implicit times, or both. Increased amplitudes or reduced implicit times are rarely seen in case of pathology. Interestingly, the ISCEV standard does not describe how much ERG parameters need to be changed to be defined as significantly changed, which is necessary for monitoring purposes. ${ }^{38}$

\section{Different types of electrodes}

To record an ERG several electrodes are necessary: a common, or ground electrode, a reference electrode, and an active electrode which may contact the cornea, bulbar
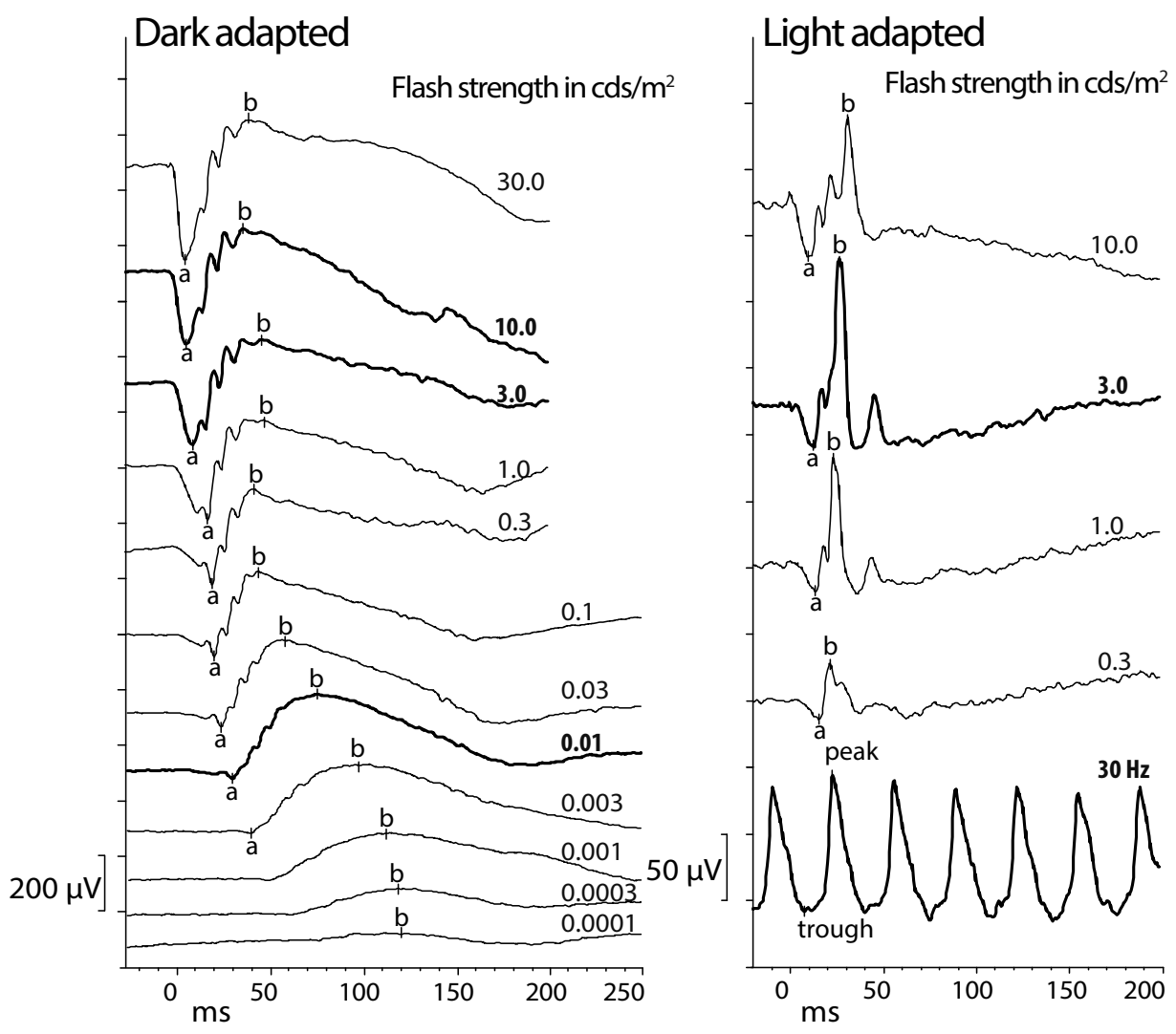

Figure 4: Example of ERG curves of a healthy control of the dark adapted and light adapted ERG of the different flash strengths. Recording time of the first seven flash strengths of the dark adapted ERG are $250 \mathrm{~ms}$. Those of the other flash strengths are 200ms. The standard ISCEV flash strengths (Dark adaptation: 0.01 (rod response), 3.0 and $10.0 \mathrm{cds} / \mathrm{m}^{2}$ (combined rod/cone response) Light adaptation: $3.0 \mathrm{cds} / \mathrm{m}^{2}$ (cone response) and $30 \mathrm{~Hz}$ (cone response)/ are indicated in bold. Abbreviations: $E R G=$ electroretinogram, $\mathrm{cds} / \mathrm{m}^{2}=$ candela - seconds/squared meters, ms $=$ milliseconds, $\mu V=$ microvolts, ISCEV $=$ International Society for Clinical Electrophysiology of Vision. 
conjunctiva, or skin of the lower eyelid. Over the years multiple types of active electrodes have been developed. Each has their own advantages and disadvantages and none is determined as the gold standard by ISCEV.

Initially, the main goal of electro-physicists was to record as high amplitudes as possible. These are best recorded at the top of cornea. ${ }^{52}$ Therefore, for a long time Burrey-Allen contactlens electrodes were the most often used electrodes. While they yield high amplitudes, the disadvantage of these electrodes is that they are quite uncomfortable for patients to wear. Also, there is a chance of damage to the cornea due to scratching.

To increase patient comfort, several other electrodes were developed. One of these is the Dawson-Trick-Litzkov (DTL) electrode. In this thesis all ERG recordings were performed with DTL electrodes. When the DTL was first introduced, its developers stated that it was possible to use it without a local anesthetic..$^{53}$ However, it is our experience that it is advisable to use some local anesthetics to ensure a better patient comfort during the ERG recording. Besides a better patient comfort, the DTL electrode is usually disposable, which decreases the possibility of an infection. Lastly, with DTL the chance of significant damage to the cornea is small.

A disadvantage of the DTL electrode is that it can shift its position during an ERG recording. The position of the DTL can affect the recorded ERG response. In chapter 6 we will look further into the effects of DTL electrode position and the ERG.

\section{Factors other than disease that can influence the ERG}

Besides retinal diseases, many other factors can influence the recorded ERG as well. Some of these factors can be (easily) addressed by adhering to the ISCEV standards. These include the duration of dark- and light adaptation, flash strength and pupil size. ${ }^{38}$

Light and dark adaptation appear to have an exponential effect on the ERG responses. In the first minutes most of the effect is reached, thereafter additional adaptation time yields only slightly larger responses. ${ }^{54}$ The standard adaptation time for dark adaptation is 20 minutes, for light adaptation 10 minutes.

Several patient specific factors can also significantly affect the recorded ERG response, but these are less important when the ERG is used for monitoring purposes, because they are expected to remain stable over the sessions. Examples of these factors are axial length, ocular pigmentation and gender. ${ }^{55-58}$

Pupil size affects how much light can reach the retina. If the amount of light that can reach the retina is reduced, this should be taken into account when an ERG is reviewed. Therefore, a recorded ERG with a small pupil size may correspond to a weaker flash strength than the one which was used. Pupil sizes were therefore recorded in the prospective studies of this thesis. 
Other factors can influence the recorded response, but are more difficult to address, include: DTL position, media opacities, age and even the time of day. ${ }^{59-64}$ Uveitis can cause several types of media opacities, including corneal keratopathy, cataracts, flare, cells in the anterior chamber, vitreous cells, and vitreous haze.1,29 These can all reduce the amount of light which reaches the retina. Posterior synechea, which can prevent the pupil form reaching full mydriasis can have a similar effect. ${ }^{43}$ However, these media opacities will often only slighty reduce the amount of light that reaches the retina, but they will all have a larger effect on light scattering. Therefore, they will have a much larger influence on the mfERG than the ffERG. However, they can all have an effect on the ERG. Therefore, media opacities are always taken into consideration in the different chapters of this thesis.

\section{LITERATURE ABOUT ELECTRORETINOGRAPHY OF UVEITIS}

The role of the ERG is unclear for most forms of uveitis but the ERG may be used for monitoring birdshot uveitis, because some studies indicate that the $30 \mathrm{~Hz}$ flicker response of the ERG is a very sensitive marker to determine if systemic medication may be tapered. ${ }^{65,66}$ Also, there are some reports that state that the ERG is useful to determine the activity of the inflammation in birdshot. ${ }^{66}$ The ERG abnormalities in birdshot uveitis may improve after the inflammation has become inactive, but there are also reports that the ERG changes may be permanent. $65,67-72$ There are indications that the ERG is helpful in predicting visual outcome in birdshot, but not all studies found a significant association. . $1,72^{2}$

However, all studies that investigate the ERG in uveitis were conducted with relative small sample sizes and often a comparison with other diagnostic tests was not made. This makes it difficult to assess the true value of the ERG in uveitis. Therefore, the ERG is not a part in the standard diagnostic work-up in the University Medical Center Utrecht. Also, the ERG is not always correlated to other tests that monitor disease activity, and its measurement takes up a long period of time. Lastly, the true value of the ERG for monitoring is not elucidated. .99,73 $^{2}$

However, ERG changes in uveitis are not restricted to birdshot uveitis. There are many reports of ERG changes in different forms of uveitis. ${ }^{74}$ Table 1 gives an overview of the different types of ERG and mfERG abnormalities that are described in uveitis. Many of these studies are case series, or studies conducted with small sample sizes. Depite the many reports of ERG abnormalities in uveitis, and the few reports that the ERG may be usefull for monitoring or prognosis, to our knowledge the ERG is not routinely used to monitor these uveitis entities. 
Table 1: overview of ERG abnormalities that are described in literature (continued on next page)

\begin{tabular}{|c|c|c|c|c|c|}
\hline & $\begin{array}{l}\text { Electoretinogram } \\
\text { Type of ERG abnormalities } \\
\text { Amplitudes } \\
\text { Reduced? }\end{array}$ & $\begin{array}{l}\text { Implicit times } \\
\text { Prolonged? }\end{array}$ & Other & $\begin{array}{l}\text { Can the } \\
\text { ERG } \\
\text { improve? }\end{array}$ & mfERG \\
\hline AMPPE & $\begin{array}{l}\text { Yes: } \\
\text { - Photopic a-wave and } \\
\text { b-wave } \\
\text { - Scotopic b-wave }\end{array}$ & & & $\mathrm{No}^{77}$ & - \\
\hline AZOOR & $\begin{array}{l}\text { Yes: } \\
\text { - 30Hz flicker }{ }^{78} \\
\text { - Photopic }{ }^{78,79,96-102} \\
\text { - Scotopic } 78,79,96-102 \\
\text { - Mesopic } \\
\text { - Undetectable } \\
\text { - Electroneg,101 }\end{array}$ & $\begin{array}{l}\text { Yes: } \\
-30 \text { Hz flicker } \\
79,98,101,102 \\
\text { - Photopic } \\
\text { - Scotopic }\end{array}$ & $\begin{array}{l}\text { - Can correlate to VF } \\
\text { loss }^{97} \\
\text { - OPs affected } \\
\text { - b/a wave ratio } \\
\text { normal79 }\end{array}$ & Yes ${ }^{79,98}$ & $\begin{array}{l}\text { - Abnormal } \\
\text { - Corresponds } \\
\text { to VF } \\
\text { defects }^{78,101}\end{array}$ \\
\hline Behcet & $\begin{array}{l}\text { Yes: }{ }^{76,103,104} \\
\text { - b-wave and scotopic first } \\
\text { affected } 103,104\end{array}$ & Yes: ${ }^{76}$ & $\begin{array}{l}\text { - 'ERG abnormal'105,106 } \\
\text { - Can predict } \\
\text { postoperative BCVA } \\
\text { after cataract surgery } \\
\text { - OPS abnormal105,106 }\end{array}$ & $-\mathrm{No}^{75}$ & $\begin{array}{l}\text { - Abnormal; } \\
\text { amp. more } \\
\text { affected than } \\
\text { IT } \\
\text { - Can } \\
\text { improve }\end{array}$ \\
\hline Birdshot & $\begin{array}{l}\text { Yes: } \\
\text { - } 30 \mathrm{~Hz} \text { flicker } 65,69,7,72,108-112 \\
\text { - Photopic } 65,68,69,7,108,11,11,114 \\
\text { - Scotopic } 65,67-69,108,109,111,113-115 \\
\text { - Mescopic } 65,67,69,70,108,109,11,115- \\
117 \\
\text { - Electronegative65,116 } \\
\text { - Almost non-recordable } \\
68,109,111\end{array}$ & 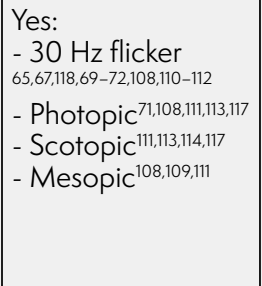 & $\begin{array}{l}\text { - OPs abnormal68,114,115 } \\
\text { - } 30 \text { Hz useful for visual } \\
\text { prognosis } 22,108,111,118 \\
\text { - Can deteriorate } \\
65,68-70,111,112,119 \\
\text { - Doesn't always } \\
\text { improve after } \\
\text { treatment } 108,112,120\end{array}$ & $\begin{array}{l}- \text { Yes 65,67- } \\
70,108,111,112\end{array}$ & $\begin{array}{l}\text { - Abnormal121 } \\
\text { - Correlated } \\
\text { to BCVA and } \\
\text { color vision } \\
\text { - Can } \\
\text { improve or } \\
\text { deteriorate }^{121}\end{array}$ \\
\hline Fuchs & $\begin{array}{l}\text { Yes: } \\
\text { - } 30 \mathrm{~Hz}_{\text {flicker }}^{95} \\
\text { - Scotopic b-wave and } \\
\text { a-wave }\end{array}$ & $\begin{array}{l}\text { Yes: } \\
\text { - } 30 \mathrm{~Hz} \text { flicker }{ }^{95} \\
\text { - Scotopic b-wave } \\
\text { and a-wave }\end{array}$ & $\begin{array}{l}\text { - OPs reduced } 95 \\
\text { - Normal123 }\end{array}$ & - & - \\
\hline IU & $\begin{array}{l}\text { Yes: } \\
\text { - } 30 \mathrm{~Hz} \text { flicker response }{ }^{89,124} \\
\text { - Photopic b-wave } \text { - }^{124} \\
\text { - Scotopic b-wave }{ }^{124} \\
\text { - Mesopic b-wave and } \\
\text { a-wave }{ }^{124} \\
\text { - Nonrecordable }\end{array}$ & $\begin{array}{l}\text { Yes: } \\
\text { - } 30 \mathrm{~Hz} \text { flicker } \\
\text { response } \text { e }^{89,124} \\
\text { - Photopic } \\
\text { b-wave } 24 \\
\text { - Mesopic b-wave } \\
\text { and a-wave }\end{array}$ & $\begin{array}{l}\text { - OPs reduced } 89,124 \\
\text { - Amplitude } 30 \mathrm{~Hz} \\
\text { flicker and DA b-wave } \\
\text { supernormal89,124 }\end{array}$ & - & - \\
\hline MEWDS & $\begin{array}{l}\text { Yes }^{80,98,125-128} \\
\text {-30 Hz flicker83 } \\
\text { - Photopic } \\
\text { - Scotopi, } \\
\text { - Mesopi, }{ }^{81} \\
\text { - Almost nonrecordable } \\
\text { - A3 }\end{array}$ & Normal'125 & $\begin{array}{l}\text {-'ERG abnormal' 82,128,129 } \\
\text { - OPs reduced } 130\end{array}$ & Yes $^{83}$ & $\begin{array}{l}\text { - Abnormal } \\
\text { - Corresponds } \\
\text { to VF80,127,131,132 } \\
\text { - Can } \\
\text { improve }\end{array}$ \\
\hline
\end{tabular}


Table 1 (continued)

\begin{tabular}{|c|c|c|c|c|c|}
\hline & $\begin{array}{l}\text { Electoretinogram } \\
\text { Type of ERG abnorma } \\
\text { Amplitudes } \\
\text { Reduced? }\end{array}$ & $\begin{array}{l}\text { ties } \\
\text { Implicit times } \\
\text { Prolonged? } \\
\end{array}$ & Other & $\begin{array}{l}\text { Can the ERG } \\
\text { improve? }\end{array}$ & mfERG \\
\hline MS & & & $\begin{array}{l}\text { MS without uveitis: } \\
\text { IT prolonged and } \\
\text { amp. reduced }{ }^{133}\end{array}$ & - & \\
\hline MFC & & & $\begin{array}{l}\text { - 'Abnormal } \\
\text { ERG'134 }\end{array}$ & - & $\begin{array}{l}\text { - Amp. abnorma|127 } \\
\text { - Can improve }{ }^{127}\end{array}$ \\
\hline POHS & $\begin{array}{l}\text { Yes: } \\
\text {-Photopic } \\
\text { - Scotopic }\end{array}$ & - & & & \\
\hline Sarcoid & $\begin{array}{l}\text { - Borderline scotopic } \\
\text { b-wave }\end{array}$ & $\begin{array}{l}\text { Yes: } \\
\text { - Photopic } \\
\text { b-wave } \\
\text { - Scotopic } \\
\text { b-wave }\end{array}$ & $\begin{array}{l}\text { - 'Abnormal } \\
\text { ERG'135 }\end{array}$ & - & Norma|136 \\
\hline Syphilis & $\begin{array}{l}\text { Yes }^{137} \text { : } \\
\text { - } 30 \text { Hz flicker } \\
\text { - Photopic } \\
\text { unrecordable } \\
\text { - Scotopic } \\
\text { - }\end{array}$ & Norma|138 & & Yes $^{94}$ & $\begin{array}{l}\text {-P1 amp. reduced }{ }^{137} \\
\text { - IT prolonged.93 } \\
\text { - nonrecordable } \\
\text { - Can improve,137 } \\
\text { recovery slow }{ }^{93,137}\end{array}$ \\
\hline $\mathrm{TB}$ & & & $\begin{array}{l}\text { - 'Abnormal } \\
\text { ERG'139 }\end{array}$ & & \\
\hline Toxoplasma & $\begin{array}{l}\text { - Photopic and } \\
\text { scotopic reduced } \\
\text { but not outside ref } \\
\text { values. }{ }^{92}\end{array}$ & Normal92 & & & \\
\hline VKH & $\begin{array}{l}Y_{e s}^{85,143} \\
\text { - 'little ERG } \\
\text { response'140 }\end{array}$ & Yes $^{143}$ & $\begin{array}{l}\text { - 'Abnormal } \\
\text { ERG'142 } \\
\text { - OPs absent141 } \\
\text { - LA useful } \\
\text { for predicting } \\
\text { postoperative } \\
\text { vision after } \\
\text { cataract surgery }{ }^{87}\end{array}$ & Yes $^{86,87,140,141,143}$ & $\begin{array}{l}\text { - Amp. and IT } \\
\text { affected }^{84,144} \\
\text { - Can improve }\end{array}$ \\
\hline
\end{tabular}

Overview of the different ERG and mfERG abnormalities in uveitis that are described in literature. If papers specify which amplitudes or implicit times are abnormal (a-wave, b-wave, DA, LA etc.), this is specified below 'yes'. If a paper specificly states that the implicit times were normal, this is noted as well, because often only amplitudes are mentioned. If papers state that the ERG was abnormal and do not specify which aspect of the ERG was abnormal (amplitudes, implicit times etc.) 'abnormal ERG' in noted in the column 'other'. Abbreviations: amp = amplitude, APMPPE = acute posterior multifocal placoid pigment epitheliopathy, AZOOR = acute zonal occult outer retinopathy, BCVA = best corrected visual acuity, DA = dark adaptation, $E R G=$ electroretinogram, IT = implicit time, $I U=$ intermediate uveitis, MS = multiple sclerosis, MFC = multifocal chorioiditis, $\mathrm{mFERG}=$ multifocal electroretinogram, MEWDS = multiple evanescent white dot syndrome, OPs $=$ occilary potentials, $\mathrm{POHS}=$ presumed ocular histoplasmosis syndrome, $T B=$ tuberculosis, VF= visual fields, $\mathrm{VKH}=$ Vogt-Koyanaga-Harada. 
It remains unclear at what stage in the disease process ERG abnormalities develop and if they are reversible or permanent. In addition, the type of ERG abnormalities vary amongst the uveitis entities. Whether there is one type of ERG abnormality which frequently occurs in all uveitis entities, remains unclear.

In Behçet's disease, it is reported that the ERG does not improve, even when the inflammation becomes quiet. ${ }^{75}$ But a low a-wave or b-wave amplitude may be useful to predict a poor outcome of cataract surgery. ${ }^{76}$

In other uveitis entities, such as in acute posterior multifocal placoid pigment epitheliopathy (APMPPE), multiple evanescent white dot syndrome (MEWDS) and acute zonal occult outer retinopathy (AZOOR), the ERG may either improve or deteriorate over time. ${ }^{77-83}$ In Vogt Koyanagi Harada (VKH) syndrome the mfERG, especially the implicit time of the $\mathrm{P1}$, may be useful to detect early retinal damage prior to peripapillary atrophy. ${ }^{84}$ Interestingly, in late stage $\mathrm{VKH}$ the implicit times of the ERG appear to be preserved, whereas the amplitudes are decreased. ${ }^{85}$ Amplitudes can improve after treatment, but unfortunately they often worsen after two years of onset. ${ }^{86}$ Similar to Behçet's disease, the ERG in VKH can also be used to predict outcome after cataract surgery. ${ }^{87}$

Abnormal implicittimes have also been described in sarcoidosis and intermediate uveitis. ${ }^{88,89}$ In childhood uveitis, ERG abnormalities are described with both amplitudes and implicit times being abnormal, even in the case of anterior uveitis. ${ }^{90}$ In older studies a 'supernormal ERG' has also been decribed; this usually indicates an abnormally large amplitude of the b-wave, but cut off points of this supernormal b-wave are not provided. In most articles the supernormal b-wave is seen in the first active stage of the disease, after which the amplitude decreases either to within reference values, or below normal.90,91 Lastly, ERG abnormalities have been described in infectious uveitis including toxoplasma chorioretinitis, ocular syphilis, and Fuchs heterochromic uveitis. ${ }^{92-95}$

Because the ERG is much more affected in retinal dystrophies than in uveitis, it may be used to distinguish uveitis from a retinal dystrophy. Using the ERG, several cases of retinal dystrophies were identified who were initially misdiagnosed as having an intermediate uveitis. ${ }^{18}$ 


\section{AIMS AND SCOPE OF THIS THESIS}

The main objective of this thesis was to investigate which ERG abnormalities occur in non-infectious uveitis. Because retinal atrophy occurs usually only after a long period of inflammation, a main objective was to investigate a possible correlation between the duration of uveitis and ERG abnormalities.

In Chapter 2 we investigate this possible correlation by measuring the ERG in two groups: uveitis patients with a disease duration of $<1$ year and $>5$ years. We also correlate clinical parameters to the ERG to investigate what factors may predict ERG abnormalities.

In Chapter $\mathbf{3}$ we look at the possible reversibility of the ERG abnormalities: a follow-up ERG was measured after 1 year in the group of patients with a short disease duration (1 year). We also correlate changes in ERG outcome to clinical parameters.

In Chapter $\mathbf{4}$ we investigated possible correlations between retinal structures and retinal function. Therefore, we segmented the OCT scans and analysed the thickness of ten retinal layers. Next, we investigated differences in retinal layer thickness based on ERG outcome.

In Chapter 5 we look further into pediatric uveitis patients with an intermediate or panuveitis who had an ERG to exclude retinal dystrophy. We investigated to what extent the ERG may be affected in childhood uveitis and what clinical factors are associated with abnormalities.

To investigate what the impact of an anterior uveitis is on retinal function, we describe the ERG of patients with a typical form of anterior uveitis: JIA-associated uveitis (Chapter 6).

In Chapter $\mathbf{7}$ we investigate the effects of DTL position on the ERG. Due to blinking the DTL may shift which may be important when patients are uncomfortable, for instance due to photophobia in uveitis patients. We repeated the LA ERG twice in our uveitis patients to see if, and to what extent amplitudes, or implicit times, or both, were affected by the DTL position. We also investigated if there is an optimal DTL location for monitoring purposes. 


\section{$\underline{\text { REFERENCES }}$}

1. Jabs D a. Standardization of uveitis nomenclature for reporting clinical data. Results of the first international workshop. Am J Ophthalmol. 2005;140(3):509-516. doi:10.1016/j. ajo.2005.03.057

2. Van der Lelij A, Rothova A. Diagnostic anterior chamber paracentesis in uveitis: a safe procedure? Br J Ophthalmol. 1997;81(11):976-979. doi:10.1136/bjo.81.11.976

3. Anwar Z, Galor A, Albini TA, Miller D, Perez V, Davis JL. The diagnostic utility of anterior chamber paracentesis with polymerase chain reaction in anterior uveitis. $\mathrm{Am} J$ Ophthalmol. 2013;155(5):781-786. doi:10.1016/j.ajo.2012.12.008

4. de Boer $\mathrm{JH}$, Verhagen C, Bruinenberg M, et al. Serologic and polymerase chain reaction analysis of intraocular fluids in the diagnosis of infectious uveitis. Am J Ophthalmol. 1996;121(6):650-658. doi:10.1016/s0002-9394(14)70631-2

5. Ten Berge JC, Schreurs MWJ, van Daele PLA, Rothova A. Autoimmunity in uveitis. Acta Ophthalmol. 2018;96(5):481-485. doi:10.1111/aos.13652

6. Rosenbaum JT, Dick AD. The Eyes Have it: A Rheumatologist's View of Uveitis. Arthritis Rheumatol (Hoboken, NJ). 2018;70(10):1533-1543. doi:10.1002/art.40568

7. Cunningham ET, Tugal-Tutkun I, Khairallah M, Okada AA, Bodaghi B, Zierhut M. Behçet Uveitis. Ocul Immunol Inflamm. 2017;25(1):2-6. doi:10.1080/09273948.2017.12 79840

8. Messenger W, Hildebrandt L, Mackensen F, Suhler E, Becker M, Rosenbaum JT. Characterisation of uveitis in association with multiple sclerosis. $\mathrm{Br} J$ Ophthalmol. 2015;99(2):205-209. doi:10.1136/bjophthalmol-2014-305518

9. Constantin T, Foeldvari I, Anton J, et al. Consensus-based recommendations for the management of uveitis associated with juvenile idiopathic arthritis: the SHARE initiative. Ann Rheum Dis. 2018;77(8):1107-1117. doi:10.1136/annrheumdis-2018-213131

10. Verhagen FH, Brouwer AH, Kuiper JJW, Ossewaarde-van Norel J, Ten Dam-van Loon $\mathrm{NH}$, de Boer JH. Potential Predictors of Poor Visual Outcome in Human Leukocyte Antigen-B27-Associated Uveitis. Am J Ophthalmol. Published online March 2016. doi:10.1016/j.ajo.2016.02.024

11. Chi C-C, Tung T-H, Wang J, et al. Risk of Uveitis Among People With Psoriasis: A Nationwide Cohort Study. JAMA Ophthalmol. 2017;135(5):415-422. doi:10.1001/ jamaophthalmol.2017.0569

12. Herbort $C P$, Pavésio $C$, LeHoang $P$, et al. Why birdshot retinochoroiditis should rather be called "HLA-A29 uveitis"? Br J Ophthalmol. 2017;101(7):851-855. doi:10.1136/ bjophthalmol-2016-309764

13. Kuiper JJW, Setten J van, Devall $M$, et al. Functionally distinct ERAP1 and ERAP2 are a hallmark of HLA-A29-(Birdshot) Uveitis. Hum Mol Genet. 2018;27(24):4333-4343. doi:10.1093/hmg/ddy319

14. Wakefield D, Yates W, Amjadi S, McCluskey P. HLA-B27 Anterior Uveitis: Immunology and Immunopathology. Ocul Immunol Inflamm. 2016;24(4):450-459. doi:10.3109/09 273948.2016.1158283

15. Horie Y, Meguro A, Ohta T, et al. HLA-B51 Carriers are Susceptible to Ocular Symptoms of Behçet Disease and the Association between the Two Becomes Stronger towards the East along the Silk Road: A Literature Survey. Ocul Immunol Inflamm. 2017;25(1):37-40. doi:10.3109/09273948.2015.1136422 
16. Rothova A, Ooijman F, Kerkhoff F, Van Der Lelij A, Lokhorst HM. Uveitis masquerade syndromes. Ophthalmology. 2001;108(2):386-399. doi:10.1016/s01616420(00)00499-1

17. Grange LK, Kouchouk A, Dalal MD, et al. Neoplastic masquerade syndromes in patients with uveitis. Am J Ophthalmol. 2014;157(3):526-531. doi:10.1016/j. ajo.2013.11.002

18. Hettinga YM, van Genderen MM, Wieringa W, Ossewaarde-van Norel J, de Boer JH. Retinal Dystrophy in 6 Young Patients Who Presented with Intermediate Uveitis. Ophthalmology. 2016;123(9):2043-2046. doi:10.1016/j.ophtha.2016.03.046

19. Sève $P$, Cacoub $P$, Bodaghi $B$, et al. Uveitis: Diagnostic work-up. A literature review and recommendations from an expert committee. Autoimmun Rev. 2017;16(12):1254-1264. doi:10.1016/j.autrev.2017.10.010

20. Richtlijn voor diagnostiek en behandeling van uveitis. Published online 2015.

21. Jabs DA. Epidemiology of uveitis. Ophthalmic Epidemiol. 15(5):283-284. doi:10.1080/09286580802478724

22. Thorne JE, Suhler E, Skup M, et al. Prevalence of Noninfectious Uveitis in the United States: A Claims-Based Analysis. JAMA Ophthalmol. 2016;134(11):1237-1245. doi:10.1001/jamaophthalmol.2016.3229

23. Wakefield D, Chang JH. Epidemiology of uveitis. Int Ophthalmol Clin. 2005;45(2):1-13.

24. Nussenblatt RB. The natural history of uveitis. Int Ophthalmol. 1990;14(5-6):303-308. doi:10.1007/bf00163549

25. Rothova A, Suttorp-van Schulten MS, Frits Treffers W, Kijlstra A. Causes and frequency of blindness in patients with intraocular inflammatory disease. $\mathrm{Br} J$ Ophthalmol. 1996;80(4):332-336.

26. Tsirouki T, Dastiridou A, Symeonidis C, et al. A Focus on the Epidemiology of Uveitis. Ocul Immunol Inflamm. 2018;26(1):2-16. doi:10.1080/09273948.2016.1196713

27. Ossewarde-van Norel A, Rothova A. Clinical review: Update on treatment of inflammatory macular edema. Ocul Immunol Inflamm. 2011;19(1):75-83. doi:10.3109/0 9273948.2010.509530

28. Dick AD, Rosenbaum JT, Al-Dhibi HA, et al. Guidance on Noncorticosteroid Systemic Immunomodulatory Therapy in Noninfectious Uveitis: Fundamentals Of Care for UveitiS (FOCUS) Initiative. Ophthalmology. 2018;125(5):757-773. doi:10.1016/j. ophtha.2017.11.017

29. Jones NP. The Manchester Uveitis Clinic: The first 3000 patients, 2: Uveitis Manifestations, Complications, Medical and Surgical Management. Ocul Immunol Inflamm. 2015;23(2):127-134. doi:10.3109/09273948.2014.968671

30. Dick AD, Tundia N, Sorg R, et al. Risk of Ocular Complications in Patients with Noninfectious Intermediate Uveitis, Posterior Uveitis, or Panuveitis. Ophthalmology. 2016;123(3):655-662. doi:10.1016/j.ophtha.2015.10.028

31. Forooghian F, Yeh S, Faia LJ, Nussenblatt RB. Uveitic foveal atrophy: clinical features and associations. Arch Ophthalmol (Chicago, III 1960). 2009;127(2):179-186. doi:10.1001/archophthalmol.2008.564 
32. Birch DG, Williams PD, Callanan D, Wang R, Locke KG, Hood DC. Macular atrophy in birdshot retinochoroidopathy: an optical coherence tomography and multifocal electroretinography analysis. Retina. 2010;30(6):930-937. doi:10.1097/ IAE.0b013e3181c720b4

33. Stunkel M, Bhattarai S, Kemerley A, et al. Vitritis in pediatric genetic retinal disorders. Ophthalmology. 2015;122(1):192-199. doi:10.1016/j.ophtha.2014.07.037

34. Yoshida N, Ikeda Y, Notomi S, et al. Clinical evidence of sustained chronic inflammatory reaction in retinitis pigmentosa. Ophthalmology. 2013;120(1):100-105. doi:10.1016/j.ophtha.2012.07.006

35. Nagpal A, Biswas J. Pseudouveitis--analysis of cases misdiagnosed as posterior uveitis. Ocul Immunol Inflamm. 2006;14(1):13-20. doi:10.1080/09273940500545627

36. Invernizzi A, Cozzi M, Staurenghi G. Optical coherence tomography and optical coherence tomography angiography in uveitis: a review. Clin Experiment Ophthalmol. Published online February 4, 2019. doi:10.1111/ceo.13470

37. Tugal-Tutkun I, Herbort CP, Khairallah M. Scoring of dual fluorescein and ICG inflammatory angiographic signs for the grading of posterior segment inflammation (dual fluorescein and ICG angiographic scoring system for uveitis). Int Ophthalmol. 2010;30(5):539-552. doi:10.1007/s10792-008-9263-x

38. McCulloch DL, Marmor MF, Brigell MG, et al. ISCEV Standard for full-field clinical electroretinography (2015 update). Doc Ophthalmol. 2015;130(1):1-12. doi:10.1007/ s10633-014-9473-7

39. Hood DC, Bach M, Brigell M, et al. ISCEV standard for clinical multifocal electroretinography (mfERG) (2011 edition). Doc Ophthalmol. 2012;124(1):1-13. doi:10.1007/s10633-011-9296-8

40. Bach $M$, Brigell MG, Hawlina $M$, et al. ISCEV standard for clinical pattern electroretinography (PERG): 2012 update. Doc Ophthalmol. 2013;126(1):1-7. doi:10.1007/s10633-012-9353-y

41. Heynen H, Wachtmeister L, van Norren D. Origin of the oscillatory potentials in the primate retina. Vision Res. 1985;25(10):1365-1373.

42. Sustar M, Holder GE, Kremers J, et al. ISCEV extended protocol for the photopic OnOff ERG. Doc Ophthalmol. Published online June 22, 2018. doi:10.1007/s10633-0189645-y

43. Perlman I. The Electroretinogram : ERG Webvision. Published 2016. Accessed February 24, 2016. http://webvision.med.utah.edu/

44. Frishman L, Sustar M, Kremers J, et al. ISCEV extended protocol for the photopic negative response (PhNR) of the full-field electroretinogram. Doc Ophthalmol. 2018;136(3):207-211. doi:10.1007/s10633-018-9638-x

45. Cvenkel B, Sustar M, Perovsek D. Ganglion cell loss in early glaucoma, as assessed by photopic negative response, pattern electroretinogram, and spectral-domain optical coherence tomography. Doc Ophthalmol. Published online May 2017. doi:10.1007/ s10633-017-9595-9

46. Robson AG, Nilsson J, Li S, et al. ISCEV guide to visual electrodiagnostic procedures. Doc Ophthalmol. 2018;136(1):1-26. doi:10.1007/s10633-017-9621-y

47. Odom JV, Bach M, Brigell M, et al. ISCEV standard for clinical visual evoked potentials: (2016 update). Doc Ophthalmol. 2016;133(1):1-9. doi:10.1007/s10633-016-9553-y 
48. Constable PA, Bach M, Frishman LJ, Jeffrey BG, Robson AG, International Society for Clinical Electrophysiology of Vision. ISCEV Standard for clinical electro-oculography (2017 update). Doc Ophthalmol. 2017;134(1):1-9. doi:10.1007/s10633-017-9573-2

49. Marmor MF, Fulton AB, Holder GE, et al. ISCEV Standard for full-field clinical electroretinography (2008 update). Doc Ophthalmol. 2009;118(1):69-77. doi:10.1007/ s10633-008-9155-4

50. Johnson MA, Jeffrey BG, Messias AM V, Robson AG. ISCEV extended protocol for the stimulus-response series for the dark-adapted full-field ERG b-wave. Doc Ophthalmol. Published online March 2019. doi:10.1007/s10633-019-09687-6

51. McCulloch DL, Kondo M, Hamilton R, et al. ISCEV extended protocol for the stimulusresponse series for light-adapted full-field ERG. Doc Ophthalmol. Published online March 2019. doi:10.1007/s10633-019-09685-8

52. SUNDMARK E. Recording of the human electroretinogram with the contact glass; influence of the position of the recording electrode on the b-potential. Acta Ophthalmol. 1958;36(2):273-280. doi:10.1111/j.1755-3768.1958.tb07710.x

53. Dawson WW, Trick GL, Litzkow CA. Improved electrode for electroretinography. Invest Ophthalmol Vis Sci. 1979;18(9):988-991.

54. Asakawa K, Ito A, Kobayashi H, Iwai A, Ito C, Ishikawa H. Adaptation time, electroretinography, and pupillography in healthy subjects. Doc Ophthalmol. Published online April 2019. doi:10.1007/s10633-019-09693-8

55. Westall CA, Dhaliwal HS, Panton CM, et al. Values of electroretinogram responses according to axial length. Doc Ophthalmol. 2001;102(2):115-130. http://www.ncbi.nlm. nih.gov/pubmed/11518455

56. Al Abdlseaed A, McTaggart Y, Ramage T, Hamilton R, McCulloch DL. Light- and darkadapted electroretinograms (ERGs) and ocular pigmentation: comparison of brownand blue-eyed cohorts. Doc Ophthalmol. 2010;121(2):135-146. doi:10.1007/s10633010-9240-3

57. Brûlé J, Lavoie M-P, Casanova C, Lachapelle P, Hébert M. Evidence of a possible impact of the menstrual cycle on the reproducibility of scotopic ERGs in women. Doc Ophthalmol. 2007;114(3):125-134. doi:10.1007/s10633-007-9045-1

58. Wan W, Chen Z, Lei B. Increase in electroretinogram rod-driven peak frequency of oscillatory potentials and dark-adapted responses in a cohort of myopia patients. Doc Ophthalmol. Published online October 28, 2019. doi:10.1007/s10633-019-09732-4

59. Kurtenbach A, Kramer S, Strasser T, Zrenner E, Langrova H. The importance of electrode position in visual electrophysiology. Doc Ophthalmol. 2017;134(2):129-134. doi:10.1007/s10633-017-9579-9

60. Fuller DG, Knighton RW, Machemer R. Bright-flash electroretinography for the evaluation of eyes with opaque vitreous. Am J Ophthalmol. 1975;80(2):214-223. http:// www.ncbi.nlm.nih.gov/pubmed/1155560

61. Kergoat $\mathrm{H}$, Kergoat MJ, Justino L. Age-related changes in the flash electroretinogram and oscillatory potentials in individuals age 75 and older. J Am Geriatr Soc. 2001;49(9):1212-1217. http://www.ncbi.nlm.nih.gov/pubmed/11559381

62. Wright CE, Williams DE, Drasdo N, Harding GF. The influence of age on the electroretinogram and visual evoked potential. Doc Ophthalmol. 1985;59(4):365-384. http://www.ncbi.nlm.nih.gov/pubmed/4028925 
63. Freund PR, Watson J, Gilmour GS, Gaillard F, Sauve Y. Differential changes in retina function with normal aging in humans. Doc Ophthalmol. 2011;122(3):177-190. doi:10.1007/s10633-011-9273-2

64. Birch DG, Berson EL, Sandberg MA. Diurnal rhythm in the human rod ERG. Invest Ophthalmol Vis Sci. 1984;25(2):236-238. http://www.ncbi.nlm.nih.gov/ pubmed/6538188

65. Holder GE, Robson AG, Pavesio C, Graham EM. Electrophysiological characterisation and monitoring in the management of birdshot chorioretinopathy. Br J Ophthalmol. 2005;89(6):709-718. doi:10.1136/bjo.2004.047837

66. Tzekov R, Madow B. Visual Electrodiagnostic Testing in Birdshot Chorioretinopathy.J Ophthalmol. 2015;2015:680215. doi:10.1155/2015/680215

67. Elbaz H, Besgen V, Rechberger K, Sekundo W, Apfelstedt-Sylla E. Electroretinogram and visual field changes in a case of birdshot chorioretinopathy. Doc Ophthalmol. 2017;134(2):149-153. doi:10.1007/s10633-017-9581-2

68. Oh KT, Christmas NJ, Folk JC. Birdshot retinochoroiditis: long term follow-up of a chronically progressive disease. Am J Ophthalmol. 2002;133(5):622-629.

69. Arya B, Westcott M, Robson AG, Holder GE, Pavesio C. Pointwise linear regression analysis of serial Humphrey visual fields and a correlation with electroretinography in birdshot chorioretinopathy. Br J Ophthalmol. 2015;99(7):973-978. doi:10.1136/ bjophthalmol-2014-306003

70. Kiss S, Ahmed M, Letko E, Foster CS. Long-term follow-up of patients with birdshot retinochoroidopathy treated with corticosteroid-sparing systemic immunomodulatory therapy. Ophthalmology. 2005;112(6):1066-1071. doi:10.1016/j.ophtha.2004.12.036

71. Touhami S, Fardeau C, Vanier A, et al. Birdshot Retinochoroidopathy: Prognostic Factors of Long-term Visual Outcome. Am J Ophthalmol. 2016;170:190-196. doi:10.1016/j.ajo.2016.08.007

72. Silpa-Archa S, Lee JJ, Boonsopon S, et al. POOR PROGNOSTIC FACTORS IN PATIENTS WITH BIRDSHOT RETINOCHOROIDOPATHY. Retina. Published online April 2016. doi:10.1097/IAE.0000000000001051

73. Comander J, Loewenstein J, Sobrin L. Diagnostic testing and disease monitoring in birdshot chorioretinopathy. Semin Ophthalmol. 2011;26(4-5):329-336. doi:10.3109/08 820538.2011.588661

74. Moschos MM, Gouliopoulos NS, Kalogeropoulos C. Electrophysiological examination in uveitis: a review of the literature. Clin Ophthalmol. 2014;8:199-214. doi:10.2147/OPTH.S54838

75. Hamza MME, Macky TA, Sidky MK, Ragab G, Soliman MM. INTRAVITREAL INFLIXIMAB IN REFRACTORY UVEITIS IN BEHCET'S DISEASE: A Safety and Efficacy Clinical Study. Retina. 2016;36(12):2399-2408. doi:10.1097/IAE.0000000000001109

76. Hu K, Lei B, Kijlstra A, et al. Male sex, erythema nodosum, and electroretinography as predictors of visual prognosis after cataract surgery in patients with Behcet disease. J Cataract Refract Surg. 2012;38(8):1382-1388. doi:10.1016/j.jcrs.2012.04.027

77. Deutman AF, Oosterhuis JA, Boen-Tan TN, Aan de Kerk AL. Acute posterior multifocal placoid pigment epitheliopathy. Pigment epitheliopathy of choriocapillaritis? Br J Ophthalmol. 1972;56(12):863-874.

78. Li D, Kishi S. Loss of photoreceptor outer segment in acute zonal occult outer retinopathy. Arch Ophthalmol (Chicago, III 1960). 2007;125(9):1194-1200. doi:10.1001/ archopht.125.9.1194 
79. Jacobson SG, Morales DS, Sun XK, et al. Pattern of retinal dysfunction in acute zonal occult outer retinopathy. Ophthalmology. 1995;102(8):1187-1198.

80. Chen D, Martidis A, Baumal CR. Transient multifocal electroretinogram dysfunction in multiple evanescent white dot syndrome. Ophthalmic Surg Lasers. 2002;33(3):246249.

81. Li D, Kishi S. Restored photoreceptor outer segment damage in multiple evanescent white dot syndrome. Ophthalmology. 2009;116(4):762-770. doi:10.1016/j. ophtha.2008.12.060

82. Jampol LM, Sieving PA, Pugh D, Fishman GA, Gilbert H. Multiple evanescent white dot syndrome. I. Clinical findings. Arch Ophthalmol (Chicago, III 1960). 1984;102(5):671674.

83. Sieving PA, Fishman GA, Jampol LM, Pugh D. Multiple evanescent white dot syndrome. II. Electrophysiology of the photoreceptors during retinal pigment epithelial disease. Arch Ophthalmol (Chicago, III 1960). 1984;102(5):675-679.

84. Chee S-P, Luu CD, Cheng C-L, Lim W-K, Jap A. Visual function in Vogt-KoyanagiHarada patients. Graefes Arch Clin Exp Ophthalmol. 2005;243(8):785-790. doi:10.1007/s00417-005-1156-3

85. da Silva FT, Hirata CE, Olivalves E, Oyamada MK, Yamamoto JH. Fundus-based and electroretinographic strategies for stratification of late-stage Vogt-KoyanagiHarada disease patients. Am J Ophthalmol. 2009;148(6):939-45.e3. doi:10.1016/j. ajo.2009.06.029

86. Sakata VM, Lavezzo MM, da Silva FT, et al. Full-field electroretinogram behavior in Vogt-Koyanagi-Harada disease: a 24-month longitudinal study in patients from acute onset evaluated with multimodal analysis. Graefes Arch Clin Exp Ophthalmol. Published online August 22, 2019. doi:10.1007/s00417-019-04440-w

87. Ji Y, Hu K, Li C, et al. Outcome and prognostic factors of phacoemulsification cataract surgery in Vogt-Koyanagi-Harada uveitis. Am J Ophthalmol. Published online September 5, 2018. doi:10.1016/j.ajo.2018.08.041

88. Koestinger A, Guex-Crosier Y, Borruat F-X. Autoimmune retinal dysfunction in sarcoid chorioretinopathy. Klin Monbl Augenheilkd. 2006;223(5):428-430. doi:10.1055/s-2006-926580

89. Cantrill HL, Ramsay RC, Knobloch WH, Purple RL. Electrophysiologic changes in chronic pars planitis. Am J Ophthalmol. 1981;91(4):505-512.

90. Shamshinova AM, Katargina LA, Orlovskaya LS. Electroretinographic findings in children with uveitis. In: Dernouchamps JP, Verougstraete C, Caspers-Velu L, Tassignon MJ, eds. Recent Advances in Uveitis. Kugler Publications, Amsterdam/ New York; 1992:397-400.

91. Ikeda H, Franchi A, Turner G, Shilling J, Graham E. Electroretinography and electrooculography to localize abnormalities in early-stage inflammatory eye disease. Doc Ophthalmol. 1989;73(4):387-394. doi:10.1007/BF00154494

92. Riemslag FC, Brinkman C), Verduyn Lunel HF, Spekreijse H, Kijlstra A. Analysis of the electroretinogram in toxoplasma retinochorioiditis. Doc Ophthalmol. 1992;82(1-2):5763.

93. Alexander P, Wen Y, Baxter JM, Tint NL, Browning AC, Amoaku WM. Visual evoked potential (VEP) and multifocal electroretinogram (mfERG) in ocular syphilitic posterior segment inflammation. Doc Ophthalmol. 2012;125(2):169-178. doi:10.1007/s10633012-9339-9 
94. Menon SR, Fleischhaver J, Jost K, Helbig H. Clinical and electrophysiological course of acute syphilitic posterior placoid chorioretinitis. Klin Monbl Augenheilkd. 2005;222(3):261-263. doi:10.1055/s-2005-857998

95. Murray DC, Stavrou P, Good PA, Murray PI. Electroretinographic findings in Fuchs' heterochromic cyclitis. Eye (Lond). 1997;11 ( Pt 1):102-108. doi:10.1038/eye.1997.20

96. Helbig $H$, Sutter $F$, Thölen A. [Acute zonal occult outer retinopathy (AZOOR)]. Ophthalmologe. 2001;98(6):574-578. http://www.ncbi.nlm.nih.gov/ pubmed/11450484

97. Gass JD, Agarwal A, Scott IU. Acute zonal occult outer retinopathy: a long-term follow-up study. Am J Ophthalmol. 2002;134(3):329-339.

98. Gass JD. Acute zonal occult outer retinopathy. Donders Lecture: The Netherlands Ophthalmological Society, Maastricht, Holland, June 19, 1992. J Clin Neuroophthalmol. 1993;13(2):79-97.

99. Kuo YC, Chen N, Tsai RK. Acute Zonal Occult Outer Retinopathy (AZOOR): a case report of vision improvement after intravitreal injection of Ozurdex. BMC Ophthalmol. 2017;17(1):236. doi:10.1186/s12886-017-0638-5

100. Spaide RF, Koizumi H, Freund KB. Photoreceptor outer segment abnormalities as a cause of blind spot enlargement in acute zonal occult outer retinopathy-complex diseases. Am J Ophthalmol. 2008;146(1):111-120. doi:10.1016/j.ajo.2008.02.027

101. Arai M, Nao-i N, Sawada A, Hayashida T. Multifocal electroretinogram indicates visual field loss in acute zonal occult outer retinopathy. Am J Ophthalmol. 1998;126(3):466469. doi:10.1016/s0002-9394(98)00117-2

102. Francis PJ, Marinescu A, Fitzke FW, Bird AC, Holder GE. Acute zonal occult outer retinopathy: towards a set of diagnostic criteria. Br J Ophthalmol. 2005;89(1):70-73. doi:10.1136/bjo.2004.042416

103. Kozousek V. [ERG and Behçet uveitis]. Ophthalmologica. 1970;161(2):196-201. doi:10.1159/000306112

104. Hatt M, Niemeyer G. [ERG in Behçet's disease (author's transl)]. Albrecht Von Graefes Arch Klin Exp Ophthalmol. 1976;198(2):113-120. http://www.ncbi.nlm.nih.gov/ pubmed/1082724

105. Asai H, Wakabayashi K, Segawa Y, Kawasaki K. [Electro-ophthalmological analysis of Behcet's disease]. Nihon Ganka Gakkai Zasshi. 1989;93(11):1038-1043.

106. Cruz RD, Adachi-Usami E, Kakisu Y. Flash electroretinograms and pattern visually evoked cortical potentials in Behçet's disease. Jpn J Ophthalmol. 1990;34(2):142-148. http://www.ncbi.nlm.nih.gov/pubmed/2214358

107. Stübiger N, Besch D, Deuter CME, Zierhut M, Kötter I. Multifocal ERG changes in patients with ocular Behçet's disease during therapy with interferon alpha 2a. Adv Exp Med Biol. 2003;528:529-532. doi:10.1007/0-306-48382-3_106

108. Zacks DN, Samson CM, Loewenstein J, Foster CS. Electroretinograms as an indicator of disease activity in birdshot retinochoroidopathy. Graefes Arch Clin Exp Ophthalmol. 2002;240(8):601-607. doi:10.1007/s00417-002-0506-7

109. Fich M, Rosenberg T. Birdshot retinochoroidopathy in monozygotic twins. Acta Ophthalmol. 1992;70(5):693-697.

110. Rush RB, Goldstein DA, Callanan DG, Meghpara B, Feuer WJ, Davis JL. Outcomes of birdshot chorioretinopathy treated with an intravitreal sustained-release fluocinolone acetonide-containing device. Am J Ophthalmol. 2011;151(4):630-636. doi:10.1016/j. ajo.2010.10.005 
111. Sobrin L, Lam BL, Liu M, Feuer WJ, Davis JL. Electroretinographic monitoring in birdshot chorioretinopathy. Am J Ophthalmol. 2005;140(1):52-64. doi:10.1016/j. ajo.2005.01.053

112. You C, Lasave AF, Kubaisi B, et al. Long-term outcomes of systemic corticosteroidsparing immunomodulatory therapy for Birdshot Retinochoroidopathy. Ocul Immunol Inflamm. Published online September 2019:1-9. doi:10.1080/09273948.2019.1641610

113. Fuerst DJ, Tessler HH, Fishman GA, Yokoyama MM, Wyhinny GJ, Vygantas CM. Birdshot retinochoroidopathy. Arch Ophthalmol (Chicago, III 1960). 1984;102(2):214219.

114. Priem HA, De Rouck A, De Laey J), Bird AC. Electrophysiologic studies in birdsho† chorioretinopathy. Am J Ophthalmol. 1988;106(4):430-436.

115. Forooghian F, Gulati N, Jabs DA. Restoration of retinal architecture following systemic immunosuppression in birdshot chorioretinopathy. Ocul Immunol Inflamm. 2010;18(6):470-471. doi:10.3109/09273948.2010.506973

116. Hirose $T$, Katsumi $O$, Pruett RC, Sakaue H, Mehta M. Retinal function in birdshot retinochoroidopathy. Acta Ophthalmol. 1991;69(3):327-337.

117. Kaplan HJ, Aaberg TM. Birdshot retinochoroidopathy. Am J Ophthalmol. 1980;90(6):773-782.

118. Thorne JE, Jabs DA, Kedhar SR, Peters GB, Dunn JP. Loss of visual field among patients with birdshot chorioretinopathy. Am J Ophthalmol. 2008;145(1):23-28. doi:10.1016/j. ajo.2007.08.039

119. Godel V, Baruch E, Lazar M. Late development of chorioretinal lesions in birdshot retinochoroidopathy. Ann Ophthalmol. 1989;21(2):49-52.

120. Doycheva D, Jägle H, Zierhut $M$, et al. Mycophenolic acid in the treatment of birdshot chorioretinopathy: long-term follow-up. Br J Ophthalmol. 2015;99(1):87-91. doi:10.1136/bjophthalmol-2014-305535

121. Mailhac A, Labarere J, Aptel F, Berthemy-Pellet S, Bouillet $L$, Chiquet C. Five-year trends in multifocal electroretinogram for patients with birdshot chorioretinopathy. Am J Ophthalmol. Published online December 14, 2018. doi:10.1016/j.ajo.2018.11.022

122. Chiquet C, Berthemy-Pellet S, Altayrac-Bethenod J, et al. Multifocal electroretinogram in birdshot chorioretinopathy. Retina. 2015;35(6):1256-1265. doi:10.1097/ IAE.0000000000000437

123. Martenet AC, Niemeyer G. [The value of electroretinography in uveitis]. Ophtalmologie. 1990;4(2):169-172.

124. Tetsuka S, Katsumi O, Mehta MC, Tetsuka H, Hirose T. Electrophysiological findings in peripheral uveitis. Ophthalmologica. 1991;203(2):89-98.

125. Aaberg TM, Campo R V, Joffe L. Recurrences and bilaterality in the multiple evanescent white-dot syndrome. Am J Ophthalmol. 1985;100(1):29-37. doi:10.1016/ s0002-9394(14)74979-7

126. Slusher MM, Weaver RG. Multiple evanescent white dot syndrome. Retina. 1988;8(2):132-135. http://www.ncbi.nlm.nih.gov/pubmed/3420313

127. Oh KT, Folk JC, Maturi RK, Moore P, Kardon RH. Multifocal electroretinography in multifocal choroiditis and the multiple evanescent white dot syndrome. Retina. 2001;21(6):581-589.

128. Hamed LM, Glaser JS, Gass JD, Schatz NJ. Protracted enlargement of the blind spot in multiple evanescent white dot syndrome. Arch Ophthalmol (Chicago, III 1960). 1989;107(2):194-198. doi:10.1001/archopht.1989.01070010200022 
129. Brown JJ, Folk JC. Current controversies in the white dot syndromes. Multifocal choroiditis, punctate inner choroidopathy, and the diffuse subretinal fibrosis syndrome. Ocul Immunol Inflamm. 1998;6(2):125-127.

130. Cheng JYC, Luu CD, Yeo IYS, Chee S-P. The outer and inner retinal function in patients with multiple evanescent white dot syndrome. Clin Experiment Ophthalmol. 2009;37(5):478-484. doi:10.1111/j.1442-9071.2009.02062.x

131. Huang HJ, Yamazaki H, Kawabata H, Ninomiya T, Adachi-Usami E. Multifocal electroretinogram in multiple evanescent white dot syndrome. Doc Ophthalmol. 92(4):301-309. doi:10.1007/bf02584084

132. Feigl B, Haas A, El-Shabrawi Y. Multifocal ERG in multiple evanescent white dot syndrome. Graefes Arch Clin Exp Ophthalmol. 2002;240(8):615-621. doi:10.1007/ s00417-002-0478-7

133. Hanson JVM, Hediger M, Manogaran P, et al. Outer Retinal Dysfunction in the Absence of Structural Abnormalities in Multiple Sclerosis. Invest Ophthalmol Vis Sci. 2018;59(1):549-560. doi:10.1167/iovs.17-22821

134. Muller W, Kleinschmid R. Zur Interpretation elektroretinographischer Befunde bei Uveitis posterior. Kiln Mbl Augenheilk. 1985;186:79-81.

135. P. Stavrou; R. Sivaraj; M. Allie; P.A. Good. Electrophysiological, Heidelberg's Retinal Tomography (HRT2) and Echographic assessment of patients with uveitis associated with sarcoidosis. Invest Ophthalmol Vis Sci. 2004;Vol.45, 27.

136. Machida S, Tanaka M, Murai K, Takahashi T, Tazawa Y. Choroidal circulatory disturbance in ocular sarcoidosis without the appearance of retinal lesions or loss of visual function. Jpn J Ophthalmol. 2004;48:392-396. doi:10.1007/s10384-004-00876

137. Joseph A, Rogers S, Browning A, et al. Syphilitic acute posterior placoid chorioretinitis in nonimmuno-compromised patients. Eye (Lond). 2007;21(8):1114-1119. doi:10.1038/ sj.eye. 6702504

138. Morgan CM, Webb RM, O'Connor GR. Atypical syphilitic chorioretinitis and vasculitis. Retina. 1984;4(4):225-231.

139. Fernandez-Sanz G, Carreno E, Mall S, Neveu MM, Holder GE, Thomas D. Unilateral Pigmented Paravenous Retinochoroidal Atrophy Associated With Presumed Ocular Tuberculosis. Ophthalmic Surg Lasers Imaging Retina. 2017;48(4):345-349. doi:10.3928/23258160-20170329-10

140. Jacobson $\mathrm{JH}$, Popkin A, Hirose T. The electroretinogram in Harada's disease. Am J Ophthalmol. 1967;64(6):1152-1154. doi:10.1016/0002-9394(67)93072-3

141. Nagaya T. Use of the electro-oculogram for diagnosing and following the development of Harada's disease. Am J Ophthalmol. 1972;74(1):99-109. doi:10.1016/0002-9394(72)91133-6

142. Souto FMS, Missaka RFBG, Magalhães BM, et al. Impact of Inflammation and Treatment on Self-reported Quality of Life in Patients with Non-acute Vogt-KoyanagiHarada Disease (VKHD). Ocul Immunol Inflamm. Published online October 31, 2019:112. doi:10.1080/09273948.2019.1668431

143. Yuan W, Zhou C, Cao Q, et al. Longitudinal study of visual function in Vogt-KoyanagiHarada Disease using full-field electroretinography. Am J Ophthalmol. Published online April 2018. doi:10.1016/j.ajo.2018.04.013 
General introduction, aims and scope

144. Yang P, Fang W, Wang L, Wen F, Wu W, Kijlstra A. Study of macular function by multifocal electroretinography in patients with Vogt-Koyanagi-Harada syndrome. Am J Ophthalmol. 2008;146(5):767-771. doi:10.1016/j.ajo.2008.05.044 

$\sqrt{(00)}(1)$ anconety and A) (1) Nolus 10

Niv

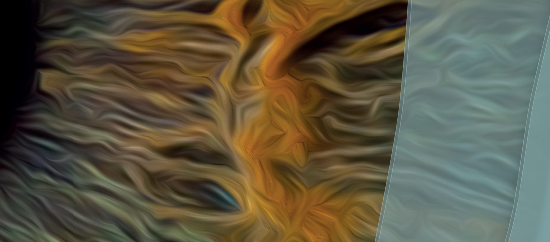

-

(

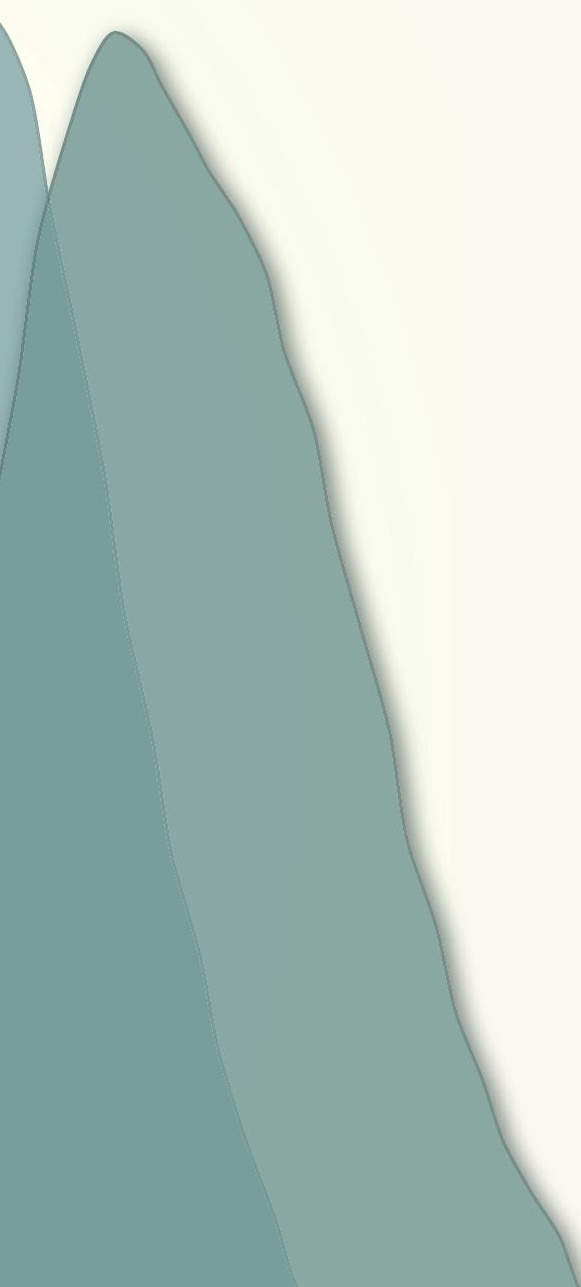

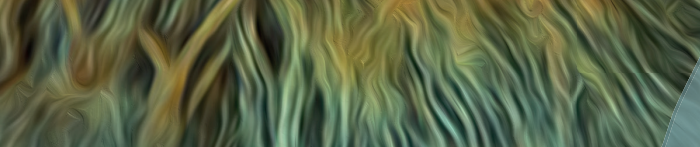
(25) (19) 


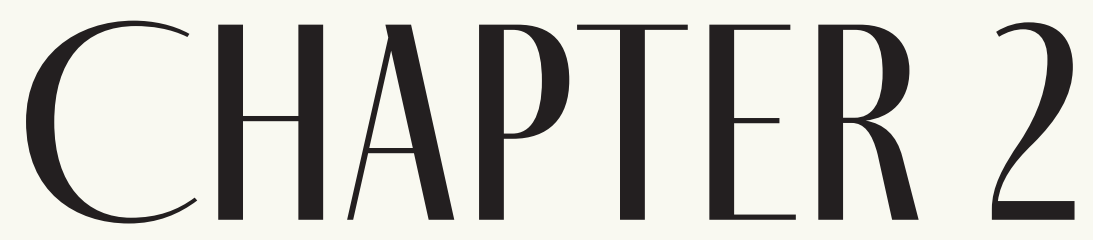

PROLONGED CONE B-WAVE ON

ELECTRORETINOGRAPHY IS ASSOCIATED

WITH SEVERITY OF INFLAMMATION IN

NONINFECTIOUS UVEITIS

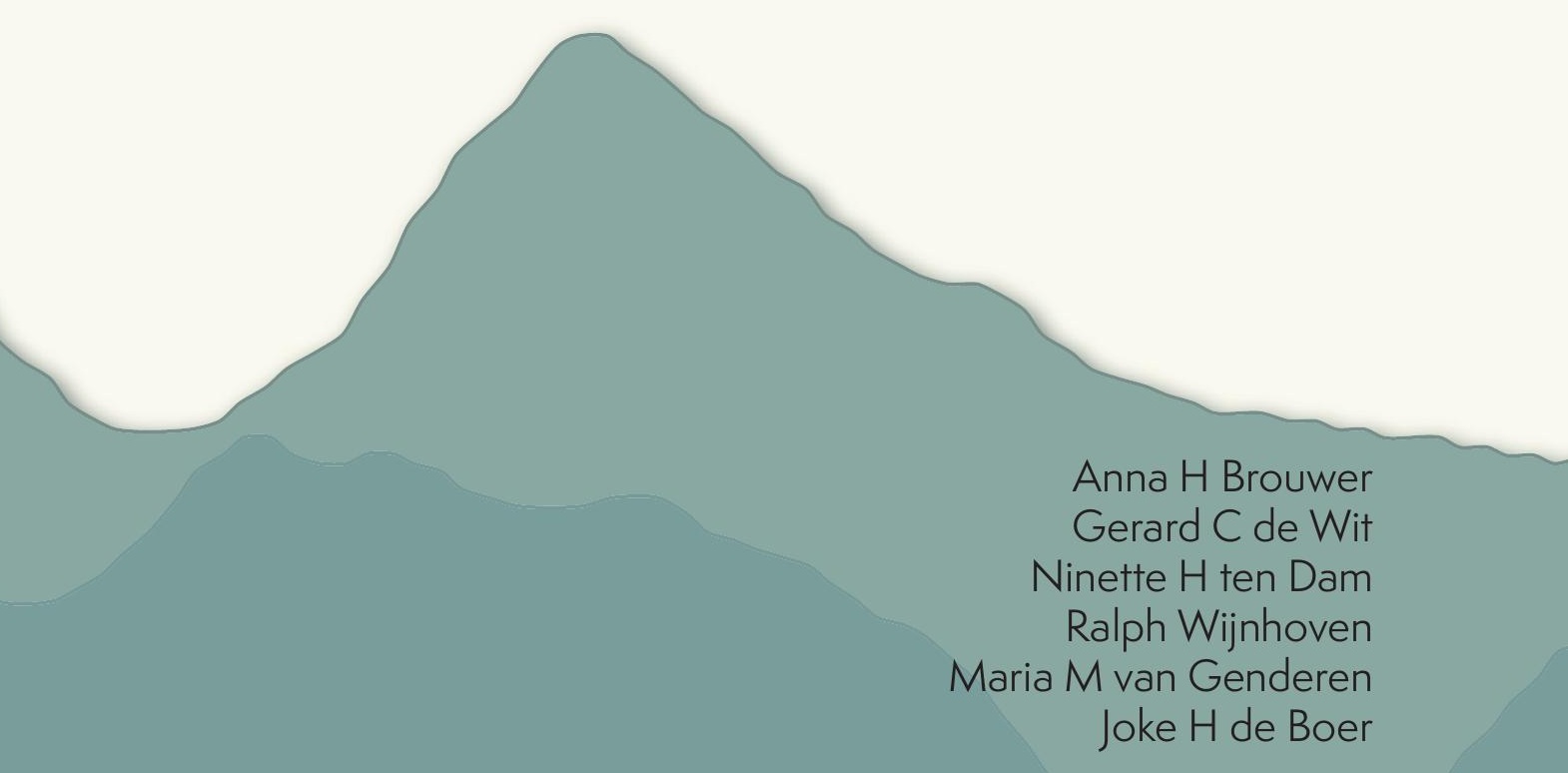

Am J Ophthalmol. 2019, doi:10.1016/j.ajo.2019.05.028 


\section{ABSTRACT}

\section{Purpose}

This study sought to investigate retinal function in patients with noninfectious uveitis by using full-field electroretinography (ERG) and correlate the ERG to disease duration and severity of inflammation.

\section{Design}

Prospective cohort study.

\section{Methods}

Patients $(n=200)$ with noninfectious uveitis and a disease duration of $<1$ year (group $A, n=80$ ) and those with a disease duration of $>5$ years (group $B, n=120$ ) were included. ERGs of the total 355 uveitis eyes were measured according to an extended International Society for Clinical Electrophysiology of Vision protocol. ERG abnormalities were related to demographics and uveitis characteristics, including anatomical classification, duration of uveitis, severity of inflammation, best corrected visual acuity (BCVA), cystoid macular edema (CME), and the highest fluorescein angiography (FA) score during the course of the disease.

\section{Results}

ERGs showed abnormalities in 235 eyes (66.2\%). The most frequent and pronounced ERG abnormality was a prolonged implicit time of the cone b-wave (151 eyes, $42.5 \%$ ), which was associated with vitritis $(p=.005)$, cells in the anterior chamber $(p=.007)$, highest FA score $(p=.011)$, age $(p<.001)$, and pupil diameter $(p<.001)$. BCVA was relatively good (0.05 LogMAR (interquartile range (IQR) $0.011-0.22)$ ) and not associated with this ERG abnormality. There were no differences between the ERG abnormalities in group $A$ and those in group $B$ and no significant associations between ERG abnormalities and the anatomical classification or specific diagnoses.

\section{Conclusions}

The ERG results are frequently affected in cases of noninfectious uveitis of all anatomic subtypes, including anterior uveitis without apparent inflammation of the posterior segment. ERG abnormalities appear to be associated with the severity of inflammation from both the present and the past and therefore may be seen even when signs of retinal inflammation have disappeared. 


\section{INTRODUCTION}

Noninfectious uveitis includes multiple diseases, some of which have an association with systemic diseases, whereas others are limited to the eye. Each of the individual diagnosis is relatively rare, and the prevalence of different subtypes varies around the world. In the United States the prevalence of uveitis is 121 cases per 100.000 adults.' Because uveitis frequently affects younger people, the social impact of the disease is high because it is responsible for approximately $10 \%$ of cases of blindness in the working population. ${ }^{2}$

Ocular inflammation may result in retinal changes, sometimes leading to retinal atrophy and functional loss. ${ }^{3}$ Most of this retinal damage occurs after the inflammation has been present for several years. With new treatment options becoming available, ophthalmologists have more opportunities to treat this disease at an early stage before structural damage occurs to the ocular tissue. ${ }^{4}$

The electroretinogram (ERG) is an objective test which measures retinal function and which is mostly used to diagnose inherited or acquired retinal diseases. It may also be used for monitoring disease and to assess the extent of retinal damage, for instance, in ocular inflammation. ${ }^{5}$ It is especially in early signs of retinal damage, prior to anatomical changes observed on optical coherence tomography (OCT), that the ERG would be of value in the care of patients.

In birdshot uveitis (BSCR) the ERG may be used for monitoring disease activity. ${ }^{6-8}$ The $30 \mathrm{~Hz}$ flicker response appears to be the most sensitive parameter, but many other ERG abnormalities can occur as well. ${ }^{7.8}$ Abnormal ERG test results have also been reported in other uveitis entities. ${ }^{9}$ However, the value of measuring ERG in inflammatory eye disease has yet to be determined.

This prospective study was conducted to gain more insight into the effects of uveitis and disease duration on ERG results. Types of ERG abnormalities in uveitis and their relation to clinical parameters were investigated.

\section{METHODS}

\section{Design and patient population}

A prospective cohort study measuring a full-field ERG in 200 patients with a noninfectious uveitis was conducted. Patients with accompanying diabetic retinopathy, retinal dystrophy, family history of retinal dystrophy, myopic degeneration, or severe media opacities were excluded. All patients were treated for uveitis at the University Medical Center Utrecht (UMCU), the Netherlands, and were $\geq 18$ years old. Patients 
were enrolled between August 2016 and February 2019. The authors approached 457 patients, 189 declined, 62 met the exclusion criteria and 6 withdrew.

It was hypothesized that there would be an effect between short-term and long-term uveitis. To evaluate the possible effect of disease duration on the ERG, two groups were created. In group A (80 patients) an ERG was measured in the first year after disease onset, and in group B (120 patients) an ERG was measured after a disease duration of $>5$ years. Disease onset was defined as the moment when an ophthalmologist first diagnosed uveitis. Patients with juvenile idiopathic arthritis (JIA) were excluded from both groups, because they were $<18$ years of age at the start of disease and therefore could not be included in group $A$.

All patients were mentally competent and gave their consent to participate. This study was conducted in compliance with the ethical principles of the declaration of Helsinki. Ethical approval was requested and obtained from the Medical Ethical Research Committee of the UMCU prior to the start of the study.

\section{ERG analysis}

ERGs were performed on the same day subjects visited the outpatient clinic when best corrected visual acuity (BCVA) and uveitis activity also were recorded. The ERGs were measured according to the International Society for Clinical Electrophysiology of Vision (ISCEV) standards. ${ }^{5}$ Dawson-Trick-Litzkow (DTL) electrodes were used as corneal electrodes and placed just below the cornea, following the lower eyelid. The use of DTLs is standard procedure in the Netherlands. Compared to contact lens electrodes, the DTL provides higher patient comfort and a lesser chance of infection, while still yielding reproducible responses. ${ }^{10}$ An Espion E3 system with colordome stimulator (Diagnosys LLC, Cambridge, UK) was used for flash stimulation.

Measurements were made according to an extended ISCEV protocol, which is standard practice in the authors' hospital. An extended protocol is useful in monitoring disease, where subtle changes must be detected. This extended protocol consists of stimuli that increase with approximately $0.5 \mathrm{log}$ units steps and range from 0.0001 $30.0 \mathrm{cds} / \mathrm{m}^{2}$ for dark-adapted (DA) ERGs and from $0.3-10.0 \mathrm{cds} / \mathrm{m}^{2}$ for light-adapted (LA) ERGs. Also a standard a $30 \mathrm{~Hz}$ flicker response (LA) was recorded.

The ERGs of the patients were compared to those of healthy controls $(n=158$; median age 47.7 years; interquartile range (IQR) 34.3 - 57.9). These reference ERGs were provided by the Rotterdam Eye Hospital and Bartiméus (Bartiméus Diagnostic Centre for complex visual disorders, Zeist) and measured with the same protocols on an Espion E2 system. Differences between the E2 and E3 system were within the measurement error. Also, the same equipment, protocols and standard operating 
procedures were used and were supervised by the same physicist, to ensure that the reference data were representative. An ERG response was considered as abnormal if amplitudes were below the $5^{\text {th }}$ percentile, or implicit times were above the $95^{\text {th }}$ percentile, or both (see Supplemental table 6 for cut off values).

ERG response abnormalities were then categorized based on the measuring conditions (LA 0.3-10.0 cds/m² (single flash cone response), LA $3.0 \mathrm{cds} / \mathrm{m}^{2} 30 \mathrm{~Hz}$ flicker response (cone response), DA $0.01 \mathrm{cds} / \mathrm{m}^{2}$ (single flash rod response), or DA 3.0-30.0 cds $/ \mathrm{m}^{2}$ (single flash combined rod/ cone response)) and which part of the response was abnormal (a-wave, b-wave, amplitudes and/or implicit times). In order for 1 of these categories to be defined as abnormal, the same type of ERG abnormality needed to be present in at least two consecutive flash strengths, except for the rod ERG, were the DA $0.01 \mathrm{cds} / \mathrm{m}^{2}$ needed to be abnormal. So, if 2 ERG responses of consecutive flash strengths of the LA ERG showed a prolonged implicit time of the $b$-wave, the implicit time of the cone b-wave was defined as abnormal. B/a wave ratios were also evaluated.

\section{Clinical parameters}

Medical records were reviewed for age, sex, medical history, uveitis diagnosis, and anatomical localization of uveitis. The patient's age at diagnosis was recorded. Uveitis activity was graded according to the Standardization of Uveitis Nomenclature criteria: cell grade in the anterior chamber, cell grade in the vitreous, flare, and vitreous haze." The presence of posterior synechiae, corneal clarity, lens clarity, macular lesions, snowballs, snow banking, vasculitis, glaucoma, and the appearance of the optic disc (hyperemia, edematous, pale) were also noted.

Additional information included whether patients were currently being treated with systemic steroids, disease-modifying antirheumatic drugs (DMARDS) (i.e. methotrexate (MTX), azathioprine, mycophenolate mofetil, mycophenolate sodium, cyclosporine, or cyclophosphamide), or biologicals (adalimumab or infliximab). Patients were also asked if they had been treated with a periocular/intraocular corticosteroid injection (betamethasone acetate $2.7 \mathrm{mg} /$ bethamethasone, or sodium phosphate 3 $\mathrm{mg}$ periocular, up to 1 month prior to ERG. Triamcinolone $40 \mathrm{mg}$ periocular, up to 3 months prior to ERG. Intravitreal dexamethasone $0.7 \mathrm{mg}$ implant, up to 3 months prior to ERG).

All patients except 2 had undergone optical coherence tomography (OCT) (Cirrus HD OCT 5000, Zeiss Meditec, Dublin, California) examination on the same day as the ERG measurement, from which the presence of cysts (cystoid macular edema (CME), sub-retinal fluid, epiretinal membranes and central retinal thickness (CRT) were noted per eye. 
If available, the mean deviation (MD) ( $n=247$ eyes) was recorded from the most recent visual field analysis (median 5 months, IQR 0 - 17 months) before the ERG measurement.

Fluorescein angiograms (FA) ( $n=333$ eyes) were scored by an experienced ophthalmologist (JdB) who was blinded regarding ERG results, using the FA scoring system of the Angiography Scoring for Uveitis Working Group (ASUWOG)12 as an indication of the severity of previous inflammation. FAs with the highest overall score until ERG measurement (median 10 months, IQR 3 - 64 months) were used for statistical analysis. When FAs were performed on the same day as the ERG ( $n=9$ patients), there was at least 1 hour between the FA and ERG.13

\section{Statistical analysis}

For statistical analysis SPSS version 25.0 (SPSS Inc., Chicago IL) was used. Only uveitis eyes were included. First, ERG results were compared to reference values, and differences between groups $A$ and group $B$ were investigated. A Mann-Whitney $U$ test was used for continuous variables and the Pearson chi squared test or the likelihood ratio for categorical variables. Using Tukey's fences $k=3$, possible outliers were identified.

Second, the most characteristic ERG abnormality result was selected and its relationship to clinical parameters was determined by using the Pearson chi-square test or the likelihood ratio for categorical variables, and a Student t-test or a MannWhitney $U$ test for continuous variables, depending on the normality of data. Normality was tested using Kolmogorov-Smirnov normality test. A Spearman rank correlation coefficient was used to find possible correlations among variables.

Third, a multiple imputation was performed to address missing values. Herein all statistically significant variables of the univariate analysis were included, as well as several auxiliary variables and the outcome variable. The automated program of SPSS was used, but continuous variables were restricted to avoid unrealistic imputed variables. The number of imputations was equal to the maximum percentage of missing data plus 1 .

Fourth, a generalized estimating equation (GEE) analysis was performed using the imputed data to correct for paired sampling of eyes and possible collinearity of parameters. Imputed data were used because a GEE analysis excludes eyes with one or more missing values, which would have led to biased results. Ordinal variables such as the amount of cells were transformed into categorical values (i.e. in the case of vitritis) to avoid complicating the model with multiple dummy variables. $P$ values below 0.05 were considered statistically significant. All tests were 2 -tailed. 


\section{$\underline{\text { RESULIS }}$}

\section{Patient characteristics}

Table 1 shows an overview of patient characteristics of both group $A$ and $B$. The median

age at ERG performance was, as expected, higher in group $B$ compared to group $A$ (54.9 years of age vs 48.4 years of age respectively, $P=.007$ ). In both groups there were more females than males. In group $B$, both eyes were more frequently affected than those in group A. However, analysis of only patients with a bilateral uveitis yielded no different results. Also, patients in group B were more frequently treated with disease-modifying antirheumatic drugs and had more frequently glaucoma. No differences were observed between groups based on diagnosis or anatomical localization of uveitis.

Table 1. Patient characteristics.

\begin{tabular}{|c|c|c|c|}
\hline & Group A (n=80) & Group B (n=120) & P-value \\
\hline Age, ${ }^{a}$ median $[I Q R]$ & $48.36[31.41,59.38]$ & $54.91[41.99,66.62]$ & 0.007 \\
\hline Male gender, $\mathrm{N}(\%)$ & $29(36.2)$ & $32(26.7)$ & 0.199 \\
\hline Bilateral uveitis, ${ }^{\circ} \mathrm{N}(\%)$ & $47(58.8)$ & $112(93.3)$ & $<0.001$ \\
\hline Localization, N (\%) & & & 0.446 \\
\hline Anterior & $5(6.2)$ & $15(12.5)$ & \\
\hline Intermediate & $9(11.2)$ & $16(13.3)$ & \\
\hline Posterior & $40(50.0)$ & $57(47.5)$ & \\
\hline Panuveitis & $26(32.5)$ & $32(26.7)$ & \\
\hline Diagnosis, N (\%) & & & 0.207 \\
\hline Behçet & $1(1.2)$ & $4(3.3)$ & \\
\hline Birdshot & $8(10.0)$ & 19 (15.8) & \\
\hline chorioretinitis $^{b}$ & $16(20.0)$ & 18 (15.0) & \\
\hline unknown & $35(43.8)$ & $43(35.8)$ & \\
\hline HLA-B27 & $5(6.2)$ & $9(7.5)$ & \\
\hline MS & $0(0.0)$ & $7(5.8)$ & \\
\hline Sarcoidosis & $14(17.5)$ & $16(13.3)$ & \\
\hline VKH & $1(1.2)$ & $4(3.3)$ & \\
\hline Oral prednisone, ${ }^{c} \mathrm{~N}(\%)$ & $34(42.5)$ & $41(34.2)$ & 0.297 \\
\hline DMARD, c N (\%) & $20(25.0)$ & $74(61.7)$ & $<0.001$ \\
\hline Biologics, ${ }^{c, d} N(\%)$ & $1(1.2)$ & $8(6.7)$ & 0.144 \\
\hline
\end{tabular}

a) At day of electroretinogram measurement. b) Includes patients with chorioiditis/chorioretinits of unknown cause, multiple evanescent white dot syndrome (MEWDS), acute multifocal placoid pigment epitheliopathy (AMPPE) and presumed ocular histoplasmosis syndrome(POHS). c) Treatment at time of ERG. dladalimumab or infliximab. Abbreviations: $I Q R$ = interquartile range, $H L A=$ human leukocyte antigen, $M S=$ multiple sclerosis, $V K H=$ Vogt-Koyanagi-Harada disease, DMARD = Disease-modifying anti-rheumatic drugs (i.e. methotrexate, azathioprine, mycophenolate mofetil, mycophenolate sodium, cyclosporine, cyclophosphamide). 


\section{ERG abnormalities}

ERG abnormalities occurred in 235 of 355 uveitis eyes (66.2\%) (Table 2). The most frequent and characteristic ERG abnormality was a prolonged implicit time of the (single- flash) cone b-wave (151 of 355 eyes (42.5\%)). An example of the cone ERG of a uveitis patient is shown in Figure 1 (A). On the right (B) an overview of the implicit time of the cone b-wave of all uveitis patients compared to healthy controls can be seen. Four eyes were identified as possible outliers for a prolonged cone b-wave. These were not excluded since they met the outlier criteria in only 1 of the 4 flash strengths, whereas the other 3 flash strengths were prolonged, but not to an outlier extent.

On a group level, the ERGs results from uveitis patients were significantly worse compared to healthy controls at almost all flash strengths. Amplitudes were lower and implicit times longer in uveitis patients compared to healthy controls (Supplemental figures 1-3 and Supplemental table 6). It was only in the DA ERG amplitudes of the lowest stimuli (DA $0.0001-0.003 \mathrm{cds} / \mathrm{m}^{2}$ ) that no statistically significant differences could be found between uveitis patients and healthy controls (Supplemental figures $1-3$, and Supplemental table 6).

A Example of prolonged cone b-wave

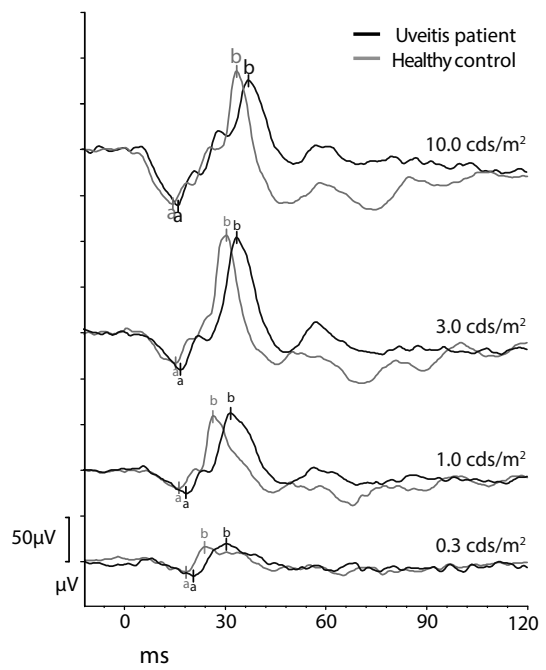

B Implicit time cone b-wave

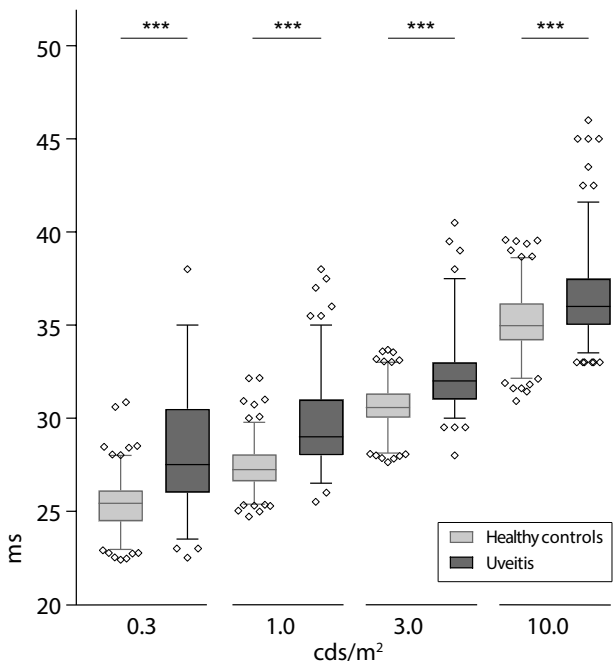

Figure 1: A) Example of a LA ERG of a patient with uveitis (black) and an ERG of an age matched control (gray). The single flash cone $b$-wave implicit times of the uveitis patient are prolonged; which is most pronounced at the lower stimulus strengths (LA 0.3 and $\left.1.0 \mathrm{cds} / \mathrm{m}^{2}\right)$. B) Boxplots of the implicit time of the single flash cone $b$-wave of different flash strengths of uveitis patients compared to those of healthy controls. Statistical testing was performed using Mann-Whitney-U test. "*** P-value of < 0.001. Abbreviations: $L A=$ light-adapted, ERG $=$ electroretinogram,$c d s / \mathrm{m}^{2}=$ candela $\cdot$ seconds $/$ squared meters, $\mathrm{ms}=$ milliseconds, $\mu \mathrm{V}=$ microvolts. 
Prolonged cone b-wave is associated with severity of inflammation

Table 2. Type and amount of electroretinogram abnormalities in uveitis eyes

\begin{tabular}{|c|c|c|c|c|c|}
\hline & All Eyes $(N=355)$ & Group A $(N=125)$ & Group B $(N=230)$ & P-value \\
\hline \multicolumn{2}{|l|}{ Overall } & $235(66.2)$ & $78(62.4)$ & $157(68.3)$ & 0.319 \\
\hline \multicolumn{2}{|c|}{ Single flash cone $e^{a, b}$} & $200(56.3)$ & $65(52.0)$ & $135(58.7)$ & 0.270 \\
\hline \multirow[t]{2}{*}{ A-wave } & Amplitude $^{b}$ & $52(14.6)$ & $17(13.6)$ & $35(15.2)$ & 0.799 \\
\hline & Implicit time ${ }^{b}$ & $121(34.1)$ & $41(32.8)$ & $80(34.8)$ & 0.795 \\
\hline \multirow[t]{2}{*}{ B-wave } & Amplitude $^{b}$ & $94(26.5)$ & $38(30.4)$ & $56(24.3)$ & 0.268 \\
\hline & Implicit time ${ }^{b}$ & $151(42.5)$ & $50(40.0)$ & 101 (43.9) & 0.549 \\
\hline \multicolumn{2}{|c|}{$30 \mathrm{~Hz}$ flicker response $\mathrm{e}^{\mathrm{a}, \mathrm{c}}$} & $167(47.0)$ & $54(43.2)$ & 113 (49.1) & 0.338 \\
\hline & Amplitude $^{c}$ & $121(34.1)$ & $36(28.8)$ & $85(37.0)$ & 0.152 \\
\hline & Implicit time ${ }^{c}$ & $123(34.6)$ & $39(31.2)$ & $84(36.5)$ & 0.374 \\
\hline \multicolumn{2}{|c|}{ Single flash rod ${ }^{c}$} & $123(34.7)$ & $41(33.1)$ & $82(35.7)$ & 0.711 \\
\hline \multirow[t]{2}{*}{ B-wave } & Amplitude $^{d}$ & $92(26.0)$ & $30(24.2)$ & $62(27.0)$ & 0.661 \\
\hline & Implicit time ${ }^{d}$ & $55(15.5)$ & $17(13.7)$ & $38(16.5)$ & 0.587 \\
\hline \multicolumn{2}{|c|}{ Single flash rod cone } & $154(43.4)$ & $52(41.6)$ & $102(44.3)$ & 0.699 \\
\hline \multirow[t]{2}{*}{ A-wave } & Amplitude & $71(20.0)$ & $25(20.0)$ & $46(20.0)$ & $<0.999$ \\
\hline & Implicit time $e^{e}$ & $86(24.2)$ & $37(29.6)$ & $49(21.3)$ & 0.107 \\
\hline \multirow[t]{2}{*}{ B-wave } & Amplitude & $72(20.3)$ & $25(20.0)$ & $47(20.4)$ & $<0.999$ \\
\hline & Implicit time & $54(15.2)$ & 20 (16.0) & 34 (14.8) & 0.880 \\
\hline
\end{tabular}

Values are in $\mathrm{N}(\%)$. a) Amount of eyes with one or more abnormalities (i.e. amplitude and/or implicit time of a-wave and/or b-wave). b/ light-adapted 0.3-10.0 cds $/ \mathrm{m}^{2}$. c) light-adapted $3.0 \mathrm{cds} / \mathrm{m}^{2}$. d) dark-adapted 0.01 cds $/ \mathrm{m}^{2}$. e) dark-adapted 3.0-30.0 cds $/ \mathrm{m}^{2}$. Abbreviations: $\mathrm{cds} / \mathrm{m}^{2}=$ candela $\cdot$ seconds $/$ squared meters.

The implicit times of the (single flash) cone b-wave were significantly correlated to the peak implicit time of the 30 flicker response (Spearman's rho coefficient .620-.814, $\mathrm{p}$-values < .001). We found no statistically significant differences in ERG abnormalities between group A and group B (Table 2).

\section{Clinical characteristics}

At time of ERG measurement, eyes in group A showed more frequently showed signs of an active inflammation than those in group $B$. The amount of cells in the vitreous was higher in group $A$ than in group $B(p<.001)$. These eyes also more frequently had CME on OCT $(20.2 \%$ vs $8.8 \%$,respectively; $p=.004)$ as well as a hyperemic optic disc as observed with fundoscopy $(23.6 \%$ vs $5.4 \%$, respectively; $p<.001)$ (Table 3$)$. 
Table 3. Inflammatory grade and other characteristics of group A and B at day of electroretinogram measurement

\begin{tabular}{|c|c|c|c|}
\hline & Group A (n=125) & Group B (n=230) & P-value \\
\hline $\begin{array}{l}\text { Duration of uveitis in years, } \\
\text { median [IQR] }\end{array}$ & $0.59[0.41,0.74]$ & $11.69[8.04,14.95]$ & $<0.001$ \\
\hline BCVA in LogMAR, median [IQR] & $0.05[-0.08,0.15]$ & $0.10[0.00,0.22]$ & 0.010 \\
\hline Mean deviation $V F^{a}$ median $[I Q R]$ & $-1.88[-3.69,-0.76]$ & $-2.45[-5.18,-0.58]$ & 0.308 \\
\hline Glaucoma, N (\%) & $6(4.8)$ & $58(25.2)$ & $<0.001$ \\
\hline CME on OCT, N (\%) & $25(20.2)$ & $20(8.8)$ & 0.004 \\
\hline Cells anterior chamber, $\mathrm{N}(\%)$ & & & 0.064 \\
\hline none & $106(86.2)$ & $206(92.0)$ & \\
\hline trace & $10(8.1)$ & $13(5.8)$ & \\
\hline $1+$ & $7(5.7)$ & $3(1.3)$ & \\
\hline $2+$ & $0(0.0)$ & $2(0.9)$ & \\
\hline Cells vitreous, $\mathrm{N}(\%)$ & & & $<0.001$ \\
\hline none & $57(46.3)$ & $147(66.2)$ & \\
\hline trace & $26(21.1)$ & $51(23.0)$ & \\
\hline $1+$ & $26(21.1)$ & $19(8.6)$ & \\
\hline $2+$ & $8(6.5)$ & $5(2.3)$ & \\
\hline 3 & $6(4.9)$ & $0(0.0)$ & \\
\hline Pale optic disc, N (\%) & $1(0.8)$ & $20(9.0)$ & 0.005 \\
\hline Hyperemic optic disc, N (\%) & $29(23.6)$ & $12(5.4)$ & $<0.001$ \\
\hline Flare, $N(\%)$ & & & 0.344 \\
\hline 0 & $114(92.7)$ & $210(93.8)$ & \\
\hline $1+$ & $6(4.9)$ & $12(5.4)$ & \\
\hline $2+$ & $3(2.4)$ & $1(0.4)$ & \\
\hline $3+$ & $0(0.0)$ & $1(0.4)$ & \\
\hline Vitreous haze, $\mathrm{N}(\%)$ & & & 0.141 \\
\hline 0 & $62(50.4)$ & $139(62.3)$ & \\
\hline $.5+$ & $42(34.1)$ & $60(26.9)$ & \\
\hline $1+$ & $17(13.8)$ & $23(10.3)$ & \\
\hline $2+$ & $2(1.6)$ & $1(0.4)$ & \\
\hline Lens/IOL opacities, $\mathrm{N}(\%)$ & $8(6.5)$ & $26(11.7)$ & 0.176 \\
\hline Steroid injection, ${ }^{b} \mathrm{~N}(\%)$ & $7(5.6)$ & $7(3.0)$ & 0.370 \\
\hline Pupil diameter, median [IQR] & $8.00[7.00,9.00]$ & $8.00[7.00,9.00]$ & 0.024 \\
\hline
\end{tabular}

a) As measured on VF analysis (Humphrey) closest to ERG. b) Treated with periocular/intraocular corticosteroid injection (betamethasone acetate $2.7 \mathrm{mg} /$ bethamethasonedisodiumphosphate $3 \mathrm{mg}$ periocular; up to $1 \mathrm{month}$ prior to $E R G$, triamcinolone $40 \mathrm{mg}$ periocular; up to 3 months prior to $E R G$, intravitreal dexamethasone 0.7 mg implant; up to 3 months prior to $E R G)$. Abbreviations: $I Q R=$ interquartile range, $B C V A=$ best corrected visual acuity, $M D=$ mean deviation, $V F=$ visual field analysis, $C M E=$ cystoid macular edema, $O C T=$ optical coherence tomography, $C R T=$ central retinal thickness, $I O L=$ intraocular lens. 
Group B eyes had evidence of more severe activity in the past, compared to group A, based on FA scores previous to ERG measurement (Supplemental table 1). BCVA was worse in group $B$ (median 0.10 LogMAR (IQR 0.00 - 0.22) vs median 0.05 LogMAR (IQR $-0.08-0.15), p=.010$ ) and the optic disc was more frequently pale compared to group A ( $9 \%$ vs $0.8 \%$, respectively; $p=.006)$. Although the total maximal FA score was not significantly higher in group $B$, the FA scores for $C M E(p<.001)$ and optic disc $(p=.010$ ) were higher in group $B$ than group $A$ (Supplemental table 1).

Interestingly, the prolonged cone $b$-wave was observed in uveitis at all anatomical locations (Supplemental table 2). Furthermore, the prolonged cone b-wave occurred in 38 eyes which were quiescent at time of ERG measurement.

\section{Multiple imputation and GEE analysis}

Because the prolonged implicit time of the cone b-wave is the most frequent and characteristic ERG abnormality, clinical parameters were studies as predictors for this abnormality.

Supplemental tables 2-4 show the results of a univariate analysis of possible predictors for a prolonged cone b-wave, based on patient characteristics and eye characteristics and FA scores. The following possible predictors were identified: age, BCVA, mean deviation on visual field analysis, pupil diameter, amount cells in the anterior chamber, amount of cells in the vitreous, vitreous haze, pale optic disc, CME on OCT, overall FA score and several subcategories of this FA score (optic disc, CME, vasculitis and capillary leakage). For further analyses, it was decided to use the overall FA score. Interestingly, an association was found with visual fields and a prolonged cone b-wave but not with glaucoma.

Most of these parameters were significantly correlated to each other (Supplemental table 5). However, Spearman's rho correlation coefficient was smaller than \pm 0.4 in all but one case. Only the correlation between BCVA and mean deviation on visual field analysis was higher than \pm 0.4 (Spearman's rho $=-0.48, p<.001$ ).

Next, a multiple imputation was performed using these possible predictors, the outcome variable (a prolonged cone b-wave) and possible auxiliary variables (sex, diagnosis and number of immunomodulating therapies). The percentage of missing values was $<5 \%$ for most variables. Except for VF, this was $30.4 \%, 17.7 \%$ for FA of the peripheral retina, and $5.4 \%$ for FA of the posterior pole.

Table 4 shows the result of the GEE analysis. Because group A and B did not differ regarding ERG abnormalities, they were analyzed as one group. Several diseasespecific factors were identified that were associated with a prolonged cone b-wave: vitritis, cells in the anterior chamber and FA score. However, age and pupil diameter were statistically significant as well. 
Age was evaluated on a per-year basis, which implied that an odds ratio (OR) of 1.059 signifies an increase in OR for each year of age. This may appear small, but because the median age of our patient population was 53.4 years, it was relevant. This effect cannot be fully explained by a lower median age of our reference values (median 47.7 years) because when patients $>60$ years of age were excluded in a separate analysis, age was still a significant predictor. To further investigate the effects of age, the mean implicit time of the cone b-wave was plotted against both uveitis eyes and reference values (Supplemental figure 4). The slope of the regression line of the implicit time versus age was much steeper in uveitis eyes at all flash strengths, which implies that there might be an additional effect of age in uveitis patients.

Because sex does not affect implicit times, a comparison was not made. ${ }^{14}$ When effects of pupil size were analyzed, uveitis eyes with a small pupil size more frequently had prolonged implicit times of the cone b-wave than healthy controls (Supplemental figure 5).

Table 4: Clinical characteristics and their association with a prolonged cone b-wave

\begin{tabular}{|c|c|c|c|c|}
\hline & OR & \multicolumn{2}{|c|}{$95 \% \mathrm{Cl}$} & P-value \\
\hline Vitreous haze & 0.757 & 0.232 & 2.477 & 0.646 \\
\hline Vitritis & 3.031 & 1.410 & 6.516 & 0.005 \\
\hline Cells anterior chamber & 4.060 & 1.457 & 11.310 & 0.007 \\
\hline FA score & 1.088 & 1.020 & 1.161 & 0.011 \\
\hline Age & 1.059 & 1.031 & 1.087 & 0.000 \\
\hline Duration of uveitis & 1.007 & 0.967 & 1.048 & 0.733 \\
\hline Pupil diameter & 0.619 & 0.478 & 0.801 & 0.000 \\
\hline Male gender & 1.219 & 0.577 & 2.574 & 0.604 \\
\hline Pale optic disc & 1.253 & 0.357 & 4.401 & 0.725 \\
\hline MD on VF & 0.966 & 0.896 & 1.043 & 0.378 \\
\hline BCVA & 1.278 & 0.486 & 3.362 & 0.619 \\
\hline CME & 1.725 & 0.622 & 4.786 & 0.295 \\
\hline
\end{tabular}

Results of a generalized estimating equation (GEE) analysis for possible predictors for a prolonged cone b-wave. Abbreviations: $O R=$ odds ratio, $C l=$ confidence interval, $F A=$ fluorescein angiogram, $M D=$ mean deviation, VF = visual field analysis, $B C V A=$ best corrected visual acuity, $C M E=$ cystoid macula edema. 
Prolonged cone b-wave is associated with severity of inflammation

\section{DISCUSSION}

The results of this study showed that ERG abnormalities occur in two-thirds of eyes with uveitis. If the ERG result was abnormal, a prolonged cone b-wave was most common. In a multivariate model it was found that the severity of inflammation, as indicated by cells in the anterior chamber, vitritis, and the highest FA score during the disease course, were predictors for this cone b-wave prolongation. In a previous study of childhood uveitis, an association was also found between cone ERG abnormalities and vitritis and CME. ${ }^{15}$ To the best of these authors' knowledge, there have been no other reports of the association between degree of inflammation and this specific cone ERG abnormality.

Generally, it is paramount to treat the inflammation instantly because a persistent inflammation has a worse visual outcome. ${ }^{16,17}$ In the present study, quiescent uveitis eyes also had a prolonged cone b-wave, suggesting that not all ERG abnormalities are reversible and that previous inflammation can lead to permanent damage.

The significant association with the highest FA score during disease course but not disease duration also underlines the importance of quickly addressing and decreasing inflammation. Why treatment was not (inversely) associated with ERG abnormalities in the present study remains speculative. The influence of treatment on the ERG changes is the subject of a current longitudinal study. Interestingly, no association was found between ERG abnormalities and the duration of uveitis, which was hypothesized.

Also, no relationship was found between ERG abnormalities and the anatomical localization of uveitis or specific diagnosis. This means that not only intermediate, posterior uveitis or panuveitis but also anterior uveitis may lead to retinal involvement. Clinically, this finding is supported by the occurrence of CME and retinal changes in more severe cases of anterior uveitis. Even in Fuchs uveitis, in which CME is uncommon, ERG abnormalities have been reported. ${ }^{18}$

The present study included patients with presumed ocular histoplasmosis syndrome and multiple evanescent white dot syndrome. There is some debate whether or not these entities should be considered forms of uveitis. However, there are reports that they are auto-inflammatory conditions and similar to other chorioretinitis entities. ${ }^{19,20}$ Furthermore, ERG abnormalities in these patients were similar to those observed in other uveitis patients, and exclusion of them did not yield different results (data not shown).

In addition to factors related to uveitis severity, age and pupil size were also significant predictors for a prolonged cone b-wave. Generally, implicit times increase with increasing age, so age was expected to be a predictor. ${ }^{21,22}$ However, the slope of 
implicit time versus age was much steeper in uveitis eyes than in reference values. This difference could not be explained by opacities of the lens or vitreous, indicating that there may be an additional uveitis effect on the ERG.

In healthy subjects, a smaller pupil size, which causes less light to fall on the retina, would result in a shorter implicit time of the cone b-wave. The strong association between smaller pupils and a more prolonged cone implicit time in the present cohort of uveitis patients therefore cannot be attributed to reduced retinal illumination but may be secondary to more active inflammation. If these eyes had reached full mydriasis, the implicit times would be even more prolonged.

The origin of the prolongation of the cone b-wave remains speculative. So far, no animal studies have investigated implicit times of the ERG in experimental autoimmune uveitis. Prolonged implicit times indicate impaired transmission of the electrical signals from the photoreceptors to the bipolar and Müller cells. One reason for impaired transmission might be damage to the synaptic structures. Evidence for synaptic damage was previously described in Behçet's disease, in which patients showed abnormalities of the outer plexiform layer on OCT. ${ }^{23}$ However, in most eye diseases, a prolongation of the implicit time is usually combined with a reduced amplitude, whereas in uveitis, the implicit time alone is frequently affected.

Why the prolongation of the cone b-wave has not been more widely reported could be due to various reasons. First, cone implicit time abnormalities were most clearly seen at dimmer stimulus strengths, which are not measured with the standard ISCEV protocol and could therefore be missed. This may also explain why the $30 \mathrm{~Hz}$ flicker response was not always affected, since the flash strength corresponds with the brighter $3.0 \mathrm{cds} / \mathrm{m}^{2}$.

Second, many reports of ERG abnormalities in uveitis focus on amplitudes. In line with previous reports, this study also found other numerous ERG abnormalities; on a group level, the ERGs of uveitis patients were worse than those of controls.

So far, it is unknown whether ERG parameters have prognostic value in uveitis. A relation was found between severity of inflammation and ERG changes, which has not been investigated in previous studies. Whether these ERG abnormalities are useful for monitoring therapy cannot be concluded from the present data, but they are subject of a current longitudinal study.

Additionally, a role for ERG in monitoring patients taking immunomodulating therapy has not been elucidated yet. Based on multiple reports with relative small sample sizes, it is said that, in birdshot uveitis, the implicit time of the $30 \mathrm{~Hz}$ flicker response is a sensitive marker for detecting early inflammation and to decide if immunotherapy is indicated or can be tapered. ${ }^{6-8,24-26}$ 
In Behçet's and Vogt Koyanagi Harada disease, the ERG results showed normalization after treatment and the LA ERG proved to be useful for predicting visual outcome after phacoemulsification. ${ }^{27-29}$ Whether improvement in ERG results will occur in the group of patients with new-onset uveitis (group A), is subject to further study. Despite these reports, perhaps not all ERG parameters have been fully explored for their prognostic value. The present authors would recommend to using the extended ISCEV protocols in uveitis and to report all ERG parameters.

A limitation of this study is that the ERG was not always performed at an early stage of disease, before treatment was started. Because this study is not longitudinal, the true prognostic value of the ERG is unknown.

The possible effects of refractive error were not analyzed, because there were no refractive data of reference values. It was assumed that the refractive value of the uveitis cohort did not differ from that in the general population or the reference cohort, because (in contrast to retinal dystrophies) no correlation exists between high refractive error and having uveitis. Individuals with myopic degeneration were excluded from this study and from reference values. Another limitation is missing values, especially of visual field analysis, which was corrected by multiple imputation.

This study highlights the fact that the ERG is frequently affected in uveitis. The study found a characteristic prolongation of the cone b-wave that was associated with more severe inflammation in both the present and the past. Remarkably, even in eyes with anterior uveitis and in quiescent uveitis eyes, the ERG could be abnormal. Further research is needed to investigate to which extent ERG abnormalities are reversible and to explore the association between ERG abnormalities and visual prognosis.

\section{$\underline{\text { ACKNOWLEDGEMENTS }}$}

All authors have completed and submitted the ICMJE form for disclosure of potential conflicts of interest and none were reported.

a. Funding/Support: The authors were supported by the following foundations: Dr. F.P Fischer Stichting, and Vereniging Bartiméus Sonneheerdt that contributed through UitZicht (the Netherlands). The funding organizations had no role in the design or conduct of this research. They provided unrestricted grants.

b. Financial Disclosures: A.H. Brouwer has no financial disclosures. G.C. de Wit is employed as a medical physicist at Bartiméus, a low vision institute in the Netherlands. Furthermore, he is a sole proprietor, with his company Optical Diagnostics (http:// www.opticaldiagnostics.com) he develops and sells ophthalmic software products and does sometimes consulting work. N.H. ten Dam has no financial disclosures. R. 
Chapter 2

Wijnhoven has no financial disclosures. J.H. de Boer was payed by Abbvie for lectures.

c. Other Acknowledgments: We would like to thank all patients for participating in the study. We thank J. Ossewarde-van Norel and L. Ho (University Medical Centre Utrecht), for their help in recruiting patients to participate in this research, D. Gültzau, Y. Burgers and M. Ballast (University Medical Centre Utrecht) for their help in recording the ERGs, F. Riemslag and H. Talsma (Bartiméus Diagnostic Centre for complex visual disorders, Zeist) for giving additional electrophysiological advice and S. Risseeuw (University Medical Centre Utrecht) for her statistical advice. 
Prolonged cone b-wave is associated with severity of inflammation

\section{$\underline{\text { REFERENCES }}$}

1. Thorne JE, Suhler E, Skup M, et al. Prevalence of Noninfectious Uveitis in the United States: A Claims-Based Analysis. JAMA Ophthalmol. 2016;134(11):1237-1245.

2. Suttorp-Schulten MS, Rothova A. The possible impact of uveitis in blindness: a literature survey. Br J Ophthalmol. 1996;80(9):844-848.

3. Forooghian F, Yeh S, Faia LJ, Nussenblatt RB. Uveitic foveal atrophy: clinical features and associations. Arch Ophthalmol (Chicago, III 1960). 2009;127(2):179-186.

4. Dick AD, Rosenbaum JT, Al-Dhibi HA, et al. Guidance on Noncorticosteroid Systemic Immunomodulatory Therapy in Noninfectious Uveitis: Fundamentals Of Care for UveitiS (FOCUS) Initiative. Ophthalmology. 2018;125(5):757-773.

5. McCulloch DL, Marmor MF, Brigell MG, et al. ISCEV Standard for full-field clinical electroretinography (2015 update). Doc Ophthalmol. 2015;130(1):1-12.

6. Tzekov R, Madow B. Visual Electrodiagnostic Testing in Birdshot Chorioretinopathy.J Ophthalmol. 2015;2015:680215.

7. Kiss S, Ahmed M, Letko E, Foster CS. Long-term follow-up of patients with birdshot retinochoroidopathy treated with corticosteroid-sparing systemic immunomodulatory therapy. Ophthalmology. 2005;112(6):1066-1071.

8. Sobrin L, Lam BL, Liu M, Feuer WJ, Davis JL. Electroretinographic monitoring in birdshot chorioretinopathy. Am J Ophthalmol. 2005;140(1):52-64.

9. Moschos MM, Gouliopoulos NS, Kalogeropoulos C. Electrophysiological examination in uveitis: a review of the literature. Clin Ophthalmol. 2014;8:199-214.

10. Beeler P, Barthelmes D, Sutter FK, Helbig H, Fleischhauer JC. Comparison of performance and patient satisfaction of two types of ERG electrodes. Klin Monbl Augenheilkd. 2007;224(4):265-268.

11. Jabs D a. Standardization of uveitis nomenclature for reporting clinical data. Results of the first international workshop. Am J Ophthalmol. 2005;140(3):509-516.

12. Tugal-Tutkun I, Herbort CP, Khairallah M. Scoring of dual fluorescein and ICG inflammatory angiographic signs for the grading of posterior segment inflammation (dual fluorescein and ICG angiographic scoring system for uveitis). Int Ophthalmol. 2010;30(5):539-552.

13. Azarmina M, Moradian S, Azarmina $\mathrm{H}$. The effect of fluorescein angiography on fullfield electroretinography parameters. J Ophthalmic Vis Res. 2012;7(4):300-304.

14. Kato K, Kondo M, Nagashima R, et al. Factors Affecting Mydriasis-Free Flicker ERGs Recorded With Real-Time Correction for Retinal Illuminance: Study of 150 Young Healthy Subjects. Invest Ophthalmol Vis Sci. 2017;58(12):5280-5286.

15. Brouwer $\mathrm{AH}$, van Genderen MM, de Wit GC, de Boer JH. Electroretinogram abnormalities in nonanterior childhood uveitis. Acta Ophthalmol. September 2018.

16. Rothova A, Suttorp-van Schulten MS, Frits Treffers W, Kijlstra A. Causes and frequency of blindness in patients with intraocular inflammatory disease. $\mathrm{Br} J$ Ophthalmol. 1996;80(4):332-336.

17. Dick AD, Tundia N, Sorg R, et al. Risk of Ocular Complications in Patients with Noninfectious Intermediate Uveitis, Posterior Uveitis, or Panuveitis. Ophthalmology. 2016;123(3):655-662.

18. Murray DC, Stavrou P, Good PA, Murray PI. Electroretinographic findings in Fuchs' heterochromic cyclitis. Eye (Lond). 1997;11 (P+1):102-108. 
19. Prasad AG, Van Gelder RN. Presumed ocular histoplasmosis syndrome. Curr Opin Ophthalmol. 2005;16(6):364-368.

20. Jampol LM, Becker KG. White spot syndromes of the retina: a hypothesis based on the common genetic hypothesis of autoimmune/inflammatory disease. Am J Ophthalmol. 2003;135(3):376-379.

21. Birch DG, Anderson JL. Standardized full-field electroretinography. Normal values and their variation with age. Arch Ophthalmol (Chicago, III 1960). 1992;110(11):1571-1576.

22. Wright CE, Williams DE, Drasdo N, Harding GF. The influence of age on the electroretinogram and visual evoked potential. Doc Ophthalmol. 1985;59(4):365-384.

23. Kido A, Uji A, Morooka S, et al. Outer Plexiform Layer Elevations as a Marker for Prior Ocular Attacks in Patients With Behcet's Disease. Invest Ophthalmol Vis Sci. 2018;59(7):2828-2832.

24. Holder GE, Robson AG, Pavesio C, Graham EM. Electrophysiological characterisation and monitoring in the management of birdshot chorioretinopathy. Br J Ophthalmol. 2005;89(6):709-718.

25. Zacks DN, Samson CM, Loewenstein J, Foster CS. Electroretinograms as an indicator of disease activity in birdshot retinochoroidopathy. Graefes Arch Clin Exp Ophthalmol. 2002;240(8):601-607.

26. Comander J, Loewenstein J, Sobrin L. Diagnostic testing and disease monitoring in birdshot chorioretinopathy. Semin Ophthalmol. 2011;26(4-5):329-336.

27. Hu K, Lei B, Kijlstra A, et al. Male sex, erythema nodosum, and electroretinography as predictors of visual prognosis after cataract surgery in patients with Behcet disease.) Cataract Refract Surg. 2012;38(8):1382-1388.

28. Ji Y, Hu K, Li C, et al. Outcome and prognostic factors of phacoemulsification cataract surgery in Vogt-Koyanagi-Harada uveitis. Am J Ophthalmol. September 2018.

29. Yuan W, Zhou C, Cao Q, et al. Longitudinal study of visual function in Vogt-KoyanagiHarada Disease using full-field electroretinography. Am J Ophthalmol. April 2018. 
Prolonged cone b-wave is associated with severity of inflammation

\section{SUPPLEMENTAL MATERIAL}

Supplemental table 1. Fluorescein angiography scores of uveitis eyes of group $A$ and $B$ (continued on next page)

\begin{tabular}{|c|c|c|c|}
\hline & Group A (n=116) & Group B (n=200) & P-value \\
\hline FA score, median [IQR] & $4.00[1.00,8.00]$ & $5.00[1.00,9.00]$ & 0.373 \\
\hline Optic disc N (\%) & & & 0.006 \\
\hline 0 & $40(33.3)$ & $73(36.5)$ & \\
\hline 1 & $24(20.0)$ & $45(22.5)$ & \\
\hline 2 & $20(16.7)$ & $53(26.5)$ & \\
\hline 3 & $36(30.0)$ & 29 (14.5) & \\
\hline Cystoid macula edema, N (\%) & & & $<0.001$ \\
\hline 0 & $68(56.7)$ & $81(40.5)$ & \\
\hline 1 & $14(11.7)$ & $9(4.5)$ & \\
\hline 2 & $7(5.8)$ & $20(10.0)$ & \\
\hline 3 & $3(2.5)$ & $27(13.5)$ & \\
\hline 4 & $28(23.3)$ & $63(31.5)$ & \\
\hline Vasculitis score, median [IQR] & $1.00[0.00,3.00]$ & $1.00[0.00,2.00]$ & 0.635 \\
\hline Vasculitis posterior pole, N (\%) & & & 0.697 \\
\hline 0 & $76(63.3)$ & $135(67.5)$ & \\
\hline 1 & $12(10.0)$ & $23(11.5)$ & \\
\hline 2 & $16(13.3)$ & $22(11.0)$ & \\
\hline 3 & $16(13.3)$ & $20(10.0)$ & \\
\hline Vasculitis quadrants, N (\%) & & & 0.081 \\
\hline 0 & $68(65.4)$ & $112(59.6)$ & \\
\hline 1 & $8(7.7)$ & $26(13.8)$ & \\
\hline 2 & $8(7.7)$ & $28(14.9)$ & \\
\hline 3 & $5(4.8)$ & $4(2.1)$ & \\
\hline 4 & $15(14.4)$ & $18(9.6)$ & \\
\hline Capp. leakage score, median [IQR] & $0.00[0.00,1.00]$ & $0.00[0.00,2.00]$ & 0.139 \\
\hline Capp. Leakage posterior pole, N (\%) & & & 0.151 \\
\hline 0 & $96(80.0)$ & $144(72.0)$ & \\
\hline 1 & $11(9.2)$ & $18(9.0)$ & \\
\hline 2 & $13(10.8)$ & 38 (19.0) & \\
\hline Focal capp. leakage quadrants, N (\%) & & & 0.595 \\
\hline 0 & $98(94.2)$ & $169(89.9)$ & \\
\hline 1 & $3(2.9)$ & $9(4.8)$ & \\
\hline 2 & $1(1.0)$ & $7(3.7)$ & \\
\hline 3 & $1(1.0)$ & $2(1.1)$ & \\
\hline 4 & $1(1.0)$ & $1(0.5)$ & \\
\hline Diffuse capp. leakage quadrants, N (\%) & & & 0.845 \\
\hline 0 & $90(86.5)$ & $155(82.4)$ & \\
\hline 2 & $3(2.9)$ & $7(3.7)$ & \\
\hline 4 & $4(3.8)$ & $11(5.9)$ & \\
\hline 6 & $0(0.0)$ & $1(0.5)$ & \\
\hline 8 & $7(6.7)$ & $14(7.4)$ & \\
\hline
\end{tabular}


Chapter 2

Supplemental table 1. (continued)

\begin{tabular}{|c|c|c|c|}
\hline & Group A (n=116) & Group B (n=200) & P-value \\
\hline Non perfusion macula, N (\%) & 104 (100.0) & $188(100.0)$ & NA \\
\hline Non perfusion posterior pole, $\mathrm{N}(\%)$ & $104(100.0)$ & $188(100.0)$ & NA \\
\hline Non perfusion quadrants, $\mathrm{N}(\%)$ & & & 0.162 \\
\hline 0 & $102(98.1)$ & $188(100.0)$ & \\
\hline 2 & $1(1.0)$ & $0(0.0)$ & \\
\hline 3 & $1(1.0)$ & $0(0.0)$ & \\
\hline Neovascularization optic disc, N (\%) & $104(100.0)$ & $188(100.0)$ & NA \\
\hline Neovascularization elsewhere, $\mathrm{N}(\%)$ & & & 0.225 \\
\hline 0 & $103(99.0)$ & $182(96.8)$ & \\
\hline 1 & $0(0.0)$ & $5(2.7)$ & \\
\hline 2 & $1(1.0)$ & $1(0.5)$ & \\
\hline Pinpoint leakage, $N(\%)$ & & & 0.059 \\
\hline 0 & $98(94.2)$ & $184(97.9)$ & \\
\hline 1 & $3(2.9)$ & $0(0.0)$ & \\
\hline 2 & $3(2.9)$ & $4(2.1)$ & \\
\hline Pooling, N (\%) & & & 0.059 \\
\hline 0 & $100(96.2)$ & $185(98.4)$ & \\
\hline 1 & $3(2.9)$ & $0(0.0)$ & \\
\hline 4 & $1(1.0)$ & $3(1.6)$ & \\
\hline
\end{tabular}

Fluorescein angiograms (FA) were scored using the Fluorescein angiographic scoring system of the Angiography Scoring for Uveitis Working Group (ASUWOG). FAs with the highest overall FA score until the $E R G$ was recorded are shown. Abbreviations: $F A=$ Fluorescein angiograms, $N=$ number, $I Q R=$ interquartile range, capp. = capillary. 
Supplemental table 2: Patient characteristics of uveitis eyes with a prolonged single flash cone b-wave

\begin{tabular}{|c|c|c|c|}
\hline & $\begin{array}{c}\text { Normal IT b-wave } \\
\qquad(\mathrm{n}=112)\end{array}$ & $\begin{array}{l}\text { Prolonged IT b-wave } \\
\qquad(\mathrm{n}=88)\end{array}$ & P-value \\
\hline Age, ${ }^{\circ}$ median $[\mathrm{IQR}]$ & $44.47[32.77,55.29]$ & $60.93[51.32,70.13]$ & $<0.001$ \\
\hline Male gender, N (\%) & $36(32.1)$ & $25(28.4)$ & 0.678 \\
\hline Bilateral uveitis, ${ }^{2} \mathrm{~N}(\%)$ & $84(75.0)$ & 75 (85.2) & 0.109 \\
\hline Localization, N (\%) & & & 0.862 \\
\hline Anterior & $13(11.6)$ & $7(8.0)$ & \\
\hline Intermediate & $14(12.5)$ & $11(12.5)$ & \\
\hline Posterior & $53(47.3)$ & $44(50.0)$ & \\
\hline Panuveitis & $32(28.6)$ & $26(29.5)$ & \\
\hline Diagnosis, N (\%) & & & 0.138 \\
\hline Behçet & $4(3.6)$ & $1(1.1)$ & \\
\hline Birdshot & $14(12.5)$ & $13(14.8)$ & \\
\hline Chorioretinitis $^{b}$ & $25(22.3)$ & $9(10.2)$ & \\
\hline unknown & $42(37.5)$ & $36(40.9)$ & \\
\hline HLA-B27 & $8(7.1)$ & $6(6.8)$ & \\
\hline MS & $5(4.5)$ & $2(2.3)$ & \\
\hline Sarcoidosis & $13(11.6)$ & $17(19.3)$ & \\
\hline VKH & $1(0.9)$ & $4(4.5)$ & \\
\hline Oral prednisone, $\mathrm{N}(\%)$ & $43(38.4)$ & $32(36.4)$ & 0.883 \\
\hline DMARD, $\mathrm{N}(\%)$ & $48(42.9)$ & $46(52.3)$ & 0.237 \\
\hline Biologics, ${ }^{c, d} \mathrm{~N}(\%)$ & $3(2.7)$ & $6(6.8)$ & 0.290 \\
\hline
\end{tabular}

Patient characteristics with a prolonged single flash cone b-wave in one or both eyes. a) At time of ERG. b) Includes patients with chorioretinits of unknown cause, multiple evanescent white dot syndrome (MEWDS), acute multifocal placoid pigment epitheliopathy (AMPPE) and presumed ocular histoplasmosis syndrome (POHS). c) Treatment at time of ERG. d) adalimumab or infliximab. Abbreviations: IT = implicit time, $E R G=$ electroretinography, $I Q R=$ interquartile range, $H L A=$ human leukocyte antigen, $M S=$ multiple sclerosis, $V K H=$ Vogt-Koyanagi-Harada disease, IMT = immunomodulating therapy (i.e. systemic corticosteroids, methotrexate, azathioprine, mycophenolate mofetil, mycophenolate sodium, cyclosporine, cyclophosphamide, adalimumab and infliximab). 
Chapter 2

Supplemental table 3. Characteristics of eyes with a prolonged single flash cone b-wave

\begin{tabular}{|c|c|c|c|}
\hline & $\begin{array}{c}\text { Normal IT b-wave } \\
\quad(n=200)\end{array}$ & $\begin{array}{l}\text { Prolonged IT b-wave } \\
\qquad(\mathrm{n}=151)\end{array}$ & P-value \\
\hline Duration of uveitis, median [IQR] & $7.56[0.72,12.37]$ & $8.27[0.73,12.74]$ & 0.221 \\
\hline BCVA, median [IQR] & $0.00[-0.08,0.10]$ & $0.15[0.05,0.30]$ & $<0.001$ \\
\hline Mean deviation VF,a median [IQR] & $-1.66[-3.17,-0.36]$ & $-3.64[-6.81,-1.61]$ & $<0.001$ \\
\hline Glaucoma, N (\%) & $32(15.7)$ & $32(21.2)$ & 0.232 \\
\hline Cystoid macula edema on OCT, N (\%) & $10(5.0)$ & $35(23.3)$ & $<0.001$ \\
\hline Cells anterior chamber, N (\%) & & & 0.027 \\
\hline none & $186(93.0)$ & $126(85.7)$ & \\
\hline trace & $7(3.5)$ & $16(10.9)$ & \\
\hline $1+$ & $5(2.5)$ & $5(3.4)$ & \\
\hline $2+$ & $2(1.0)$ & $0(0.0)$ & \\
\hline Cells vitreous, $\mathrm{N}(\%)$ & & & 0.002 \\
\hline none & $134(67.0)$ & $70(48.3)$ & \\
\hline trace & $42(21.0)$ & $35(24.1)$ & \\
\hline $1+$ & $18(9.0)$ & $27(18.6)$ & \\
\hline $2+$ & $4(2.0)$ & $9(6.2)$ & \\
\hline 3 & $2(1.0)$ & $4(2.8)$ & \\
\hline Pale optic disc, N (\%) & $6(3.0)$ & $15(10.3)$ & 0.010 \\
\hline Hyperemic optic disc, N (\%) & $23(11.5)$ & $18(12.3)$ & 0.946 \\
\hline Flair, N (\%) & & & 0.412 \\
\hline none & $190(95.0)$ & $134(91.2)$ & \\
\hline faint & $8(4.0)$ & $10(6.8)$ & \\
\hline moderate & $2(1.0)$ & $2(1.4)$ & \\
\hline marked & $0(0.0)$ & $1(0.7)$ & \\
\hline Vitreous haze, N (\%) & & & 0.007 \\
\hline none & $131(65.5)$ & $70(47.9)$ & \\
\hline faint & $46(23.0)$ & $56(38.4)$ & \\
\hline moderate & 22 (11.0) & $18(12.3)$ & \\
\hline marked & $1(0.5)$ & $2(1.4)$ & \\
\hline Lens/IOL opacities, N (\%) & $15(7.5)$ & $19(12.9)$ & 0.138 \\
\hline Pupil diameter, median [IQR] & $8.00[8.00,9.00]$ & $7.00[6.00,8.00]$ & $<0.001$ \\
\hline
\end{tabular}

a) Closest to ERG. Abbreviations: $E R G=$ electroretinography, $I Q R=$ interquartile range, $B C V A=$ best corrected visual acuity, IT = implicit time, $M D=$ mean deviation, $V F=$ visual field analysis, $C M E=$ cystoid macular edema, $O C T=$ optical coherence tomography, $C R T=$ central retinal thickness, $I O L=$ intraocular lens 
Supplemental table 4. FA scores of eyes with a prolonged single flash cone b-wave (continued on next page)

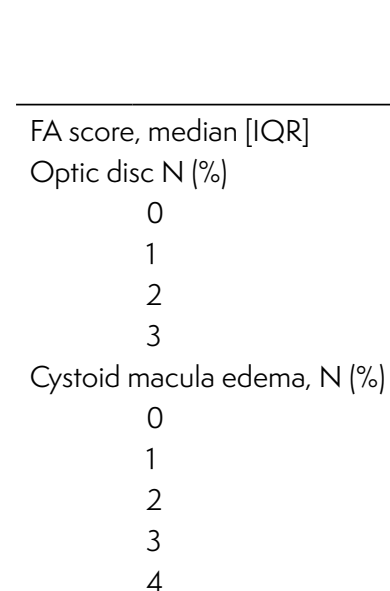

Vasculitis score, median [IQR]

Vasculitis posterior pole, $\mathrm{N}(\%)$

$$
\begin{aligned}
& 0 \\
& 1 \\
& 2 \\
& 3
\end{aligned}
$$

Vasculitis quadrants, $\mathrm{N}(\%)$

$$
\begin{aligned}
& 0 \\
& 1 \\
& 2 \\
& 3 \\
& 4
\end{aligned}
$$

Capp. leakage score, median [IQR]

Capp. leakage posterior pole, $\mathrm{N}(\%)$

$$
1
$$$$
0
$$

Focal capp. leakage quadrants, $\mathrm{N}(\%)$

$$
\begin{aligned}
& 0 \\
& 1 \\
& 2 \\
& 3 \\
& 4
\end{aligned}
$$

Diffuse capp. leakage quadrants, $N(\%)$

$$
\begin{aligned}
& 0 \\
& 2 \\
& 4 \\
& 6 \\
& 8
\end{aligned}
$$

\begin{tabular}{ccc}
$\begin{array}{c}\text { Normal IT b-wave } \\
(n=204)\end{array}$ & $\begin{array}{c}\text { Prolonged IT b-wave } \\
(n=151)\end{array}$ & P-value \\
\hline $4.00[0.00,7.75]$ & $7.00[4.00,11.00]$ & $<0.001$ \\
& & 0.008
\end{tabular}

$76(42.5)$
$40(22.3)$
$33(18.4)$
$30(16.8)$
$111(62.0)$
$11(6.1)$
$13(7.3)$
$12(6.7)$
$32(17.9)$
$0.00[0.00,2.00]$

$$
\begin{aligned}
& 37(26.2) \\
& 29(20.6) \\
& 40(28.4) \\
& 35(24.8)
\end{aligned}
$$$$
38 \text { (27.0) }
$$$$
12(8.5)
$$$$
14(9.9)
$$$$
18(12.8)
$$$$
59(41.8)
$$$$
1.00[0.00,3.00] \quad 0.002
$$$$
0.021
$$

$131(73.2)$

$15(8.4)$

$16(8.9)$

$17(9.5)$

$80(56.7)$

$20(14.2)$

22 (15.6)

19 (13.5)

$<0.001$

$115(67.6)$

$13(7.6)$

18 (10.6)

$8(4.7)$

$16(9.4)$

$0.00[0.00,0.75]$

$146(81.6)$

$15(8.4)$

18 (10.1)

$163(95.9)$

$3(1.8)$

$2(1.2)$

$1(0.6)$

$1(0.6)$

$150(88.2)$

$6(3.5)$

$8(4.7)$

$1(0.6)$

$5(2.9)$

$65(53.3)$

21 (17.2)

18 (14.8)

$1(0.8)$

17 (13.9)

0.008

$0.00[0.00,3.00] \quad<0.001$

94 (66.7)

$14(9.9)$

$33(23.4)$

0.029

$104(85.2)$

$9(7.4)$

$6(4.9)$

$2(1.6)$

1 (0.8)

95 (77.9)

4 (3.3)

$7(5.7)$

$0(0.0)$

$16(13.1)$ 
Chapter 2

Supplemental table 4 (continued)

\begin{tabular}{|c|c|c|c|}
\hline & $\begin{array}{c}\text { normal IT b-wave } \\
\quad(n=204)\end{array}$ & $\begin{array}{l}\text { prolonged IT b-wave } \\
\qquad(\mathrm{n}=151)\end{array}$ & P-value \\
\hline Non perfusion macula, $\mathrm{N}(\%)$ & $170(100.0)$ & $122(100.0)$ & NA \\
\hline Non perfusion posterior pole, $\mathrm{N}(\%)$ & $170(100.0)$ & $122(100.0)$ & NA \\
\hline Non perfusion quadrants, $\mathrm{N}(\%)$ & & & 0.348 \\
\hline 0 & $169(99.4)$ & $121(99.2)$ & \\
\hline 2 & $0(0.0)$ & $1(0.8)$ & \\
\hline 3 & $1(0.6)$ & $0(0.0)$ & \\
\hline Neovascularization optic disc, N (\%) & $170(100.0)$ & $122(100.0)$ & NA \\
\hline Neovascularization elsewhere, $\mathrm{N}(\%)$ & & & 0.152 \\
\hline 0 & $166(97.6)$ & $119(97.5)$ & \\
\hline 1 & $4(2.4)$ & $1(0.8)$ & \\
\hline 2 & $0(0.0)$ & $2(1.6)$ & \\
\hline Pinpoint leakage, N (\%) & & & 0.474 \\
\hline 0 & $166(97.6)$ & $116(95.1)$ & \\
\hline 1 & $1(0.6)$ & $2(1.6)$ & \\
\hline 2 & $3(1.8)$ & $4(3.3)$ & \\
\hline Pooling, N (\%) & & & 0.097 \\
\hline 0 & $167(98.2)$ & $118(96.7)$ & \\
\hline 1 & $0(0.0)$ & $3(2.5)$ & \\
\hline 4 & $3(1.8)$ & $1(0.8)$ & \\
\hline
\end{tabular}

Fluorescein angiograms (FA) were scored using the Fluorescein angiographic scoring system of the Angiography Scoring for Uveitis Working Group (ASUWOG). FAs with the highest overall FA score until the $E R G$ was recorded are shown. Abbreviations: $F A=$ Fluorescein angiogram, $I T=$ implicit time, $N=$ number, capp. = capillary. 
Supplemental table 5: Spearman's rho correlation coefficient of possible predictors for a prolonged single flash cone b-wave

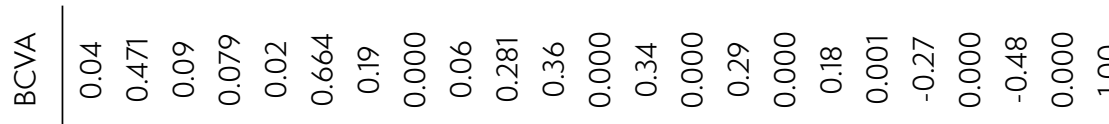

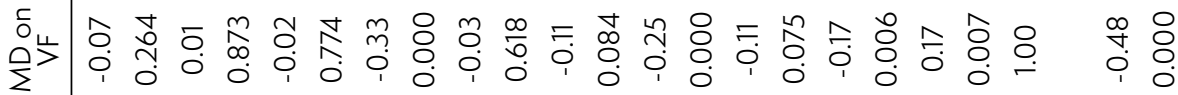

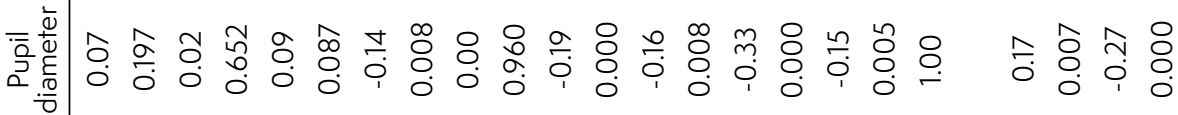

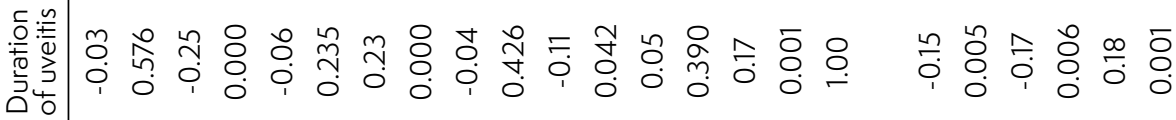

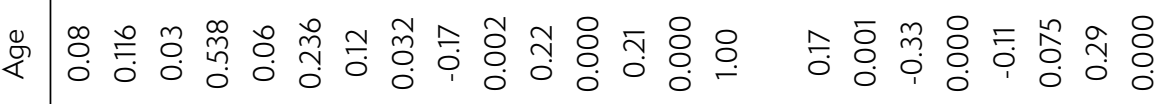

产

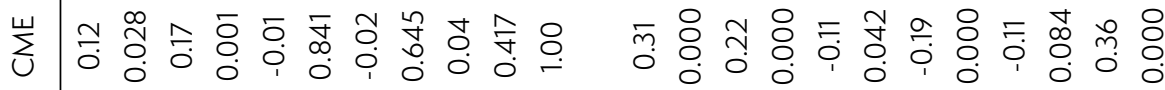

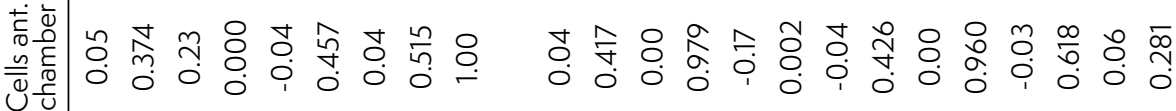

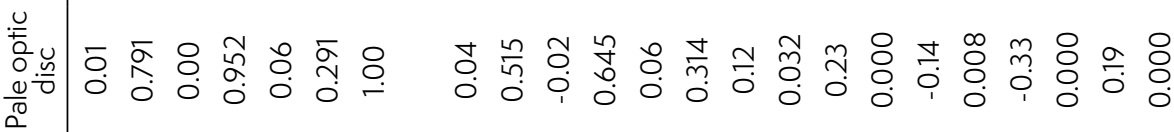

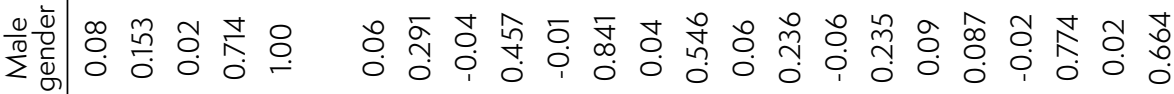

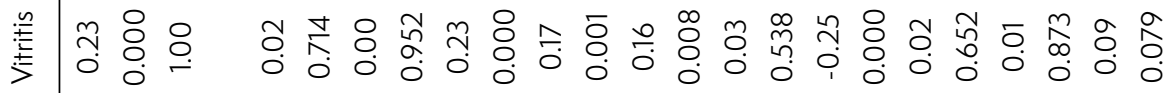

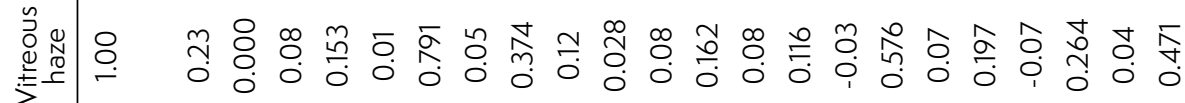

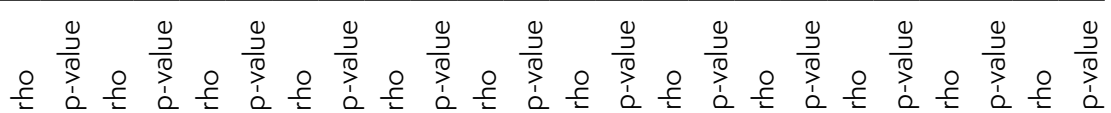

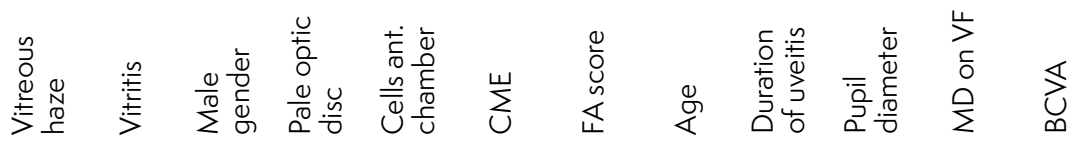


Supplemental table 6: Cut off values and overview of reference values and uveitis patients (continued on next page)

\begin{tabular}{|c|c|c|c|c|c|}
\hline & & $\begin{array}{l}\text { Cut off } \\
\text { value }\end{array}$ & $\underset{\text { median }}{\mathrm{HC}}[\mathrm{IQR}]$ & $\begin{array}{c}\text { Uveitis } \\
\text { median [IQR] }\end{array}$ & P-value \\
\hline & A-wave amplitude & & & & \\
\hline & 0.3 & -5.17 & $-12.35[-17.01,-8.38]$ & $-11.80[-15.49,-8.13]$ & 0.046 \\
\hline & 1.0 & -10.67 & $-21.28[-26.93,-16.45]$ & $-19.07[-25.13,-13.80]$ & $<0.001$ \\
\hline & 3.0 & -21.31 & $-36.13[-44.15,-29.10]$ & $-29.59[-37.73,-22.11]$ & $<0.001$ \\
\hline & 10.0 & -31.27 & $-52.58[-63.13,-41.90]$ & $-45.98[-57.64,-35.24]$ & $<0.001$ \\
\hline & A-wave implicit time & & & & \\
\hline & 0.3 & 19.65 & $17.64[16.81,18.50]$ & $19.00[18.50,20.00]$ & $<0.001$ \\
\hline & 1.0 & 17.55 & $15.87[15.07,16.50]$ & $17.00[16.50,18.00]$ & $<0.001$ \\
\hline & 3.0 & 16.16 & $14.98[14.34,15.44]$ & $16.00[15.00,16.50]$ & $<0.001$ \\
\hline & 10.0 & 15.12 & $13.91[13.45,14.45]$ & $15.00[14.50,15.50]$ & $<0.001$ \\
\hline $\begin{array}{l}\text { 总 } \\
\text { d }\end{array}$ & B-wave amplitude & & & & \\
\hline$\frac{\sigma}{\hbar}$ & 0.3 & 21.50 & $36.31[28.86,46.11]$ & $32.19[22.84,40.91]$ & $<0.001$ \\
\hline $\begin{array}{l}0 \\
0\end{array}$ & 1.0 & 55.45 & $92.92[76.76,116.80]$ & $74.12[53.73,96.21]$ & $<0.001$ \\
\hline$\frac{5}{.5}$ & 3.0 & 92.12 & $139.40[116.28,170.70]$ & $116.60[89.54,151.05]$ & $<0.001$ \\
\hline & 10.0 & 78.40 & $129.00[105.60,160.87]$ & $117.20[91.06,144.20]$ & $<0.001$ \\
\hline & B-wave implicit time & & & & \\
\hline & 0.3 & 27.62 & $25.43[24.47,26.10]$ & $27.50[26.00,30.50]$ & $<0.001$ \\
\hline & 1.0 & 29.32 & $27.24[26.61,28.05]$ & $29.00[28.00,31.00]$ & $<0.001$ \\
\hline & 3.0 & 32.57 & $30.56[30.02,31.33]$ & $32.00[31.00,33.00]$ & $<0.001$ \\
\hline & 10.0 & 38.16 & $34.96[34.17,36.17]$ & $36.00[35.00,37.50]$ & $<0.001$ \\
\hline & $30 \mathrm{~Hz}$ flicker response & & & & \\
\hline & Amplitude peak & 52.37 & $88.35[67.86,110.53]$ & $63.86[46.11,82.97]$ & $<0.001$ \\
\hline & Implicit Time Trough & 23.16 & $20.34[19.77,21.60]$ & $22.50[21.00,23.50]$ & $<0.001$ \\
\hline & Implicit Time Peak & 30.38 & $27.31[26.45,28.31]$ & $29.50[28.00,31.50]$ & $<0.001$ \\
\hline & A-wave amplitude & & & & \\
\hline & 0.0001 & & $-5.36[-10.00,-2.34]$ & $0.00[0.00,0.00]$ & $<0.001$ \\
\hline & 0.0003 & & $-6.31[-10.59,-2.60]$ & $0.00[-3.64,3.37]$ & $<0.001$ \\
\hline & 0.001 & & $-7.72[-13.32,-3.99]$ & $-0.90[-5.04,3.27]$ & $<0.001$ \\
\hline $\begin{array}{l}\text { O } \\
\text { य }\end{array}$ & 0.003 & & $-10.82[-18.36,-4.92]$ & $-3.23[-10.94,0.71]$ & $<0.001$ \\
\hline 崖 & 0.01 & & $-19.76[-29.95,-13.03]$ & $-13.47[-22.97,-3.99]$ & $<0.001$ \\
\hline $\begin{array}{l}\frac{1}{0} \\
\frac{0}{0} \\
0\end{array}$ & 0.03 & & $-38.58[-52.41,-27.54]$ & $-26.75[-41.47,-15.70]$ & $<0.001$ \\
\hline$\stackrel{0}{*}$ & 0.1 & & $-63.94[-84.69,-43.96]$ & $-41.59[-60.39,-27.88]$ & $<0.001$ \\
\hline 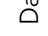 & 0.3 & & $-115.90[-148.40,-90.46]$ & $-77.55[-112.10,-52.44]$ & $<0.001$ \\
\hline & 1.0 & & $-182.85[-228.60,-153.10]$ & $-143.30[-194.55,-107.45]$ & $<0.001$ \\
\hline & 3.0 & -131.15 & $-227.90[-277.98,-188.20]$ & $-178.60[-227.45,-136.85]$ & $<0.001$ \\
\hline & 10.0 & -176.30 & $-266.80[-328.55,-229.50]$ & $-229.40[-279.70,-178.30]$ & $<0.001$ \\
\hline & 30.0 & -177.93 & $-274.00[-337.20,-234.05]$ & $-240.20[-290.45,-191.05]$ & $<0.001$ \\
\hline
\end{tabular}

Cut off values were based on reference values. An amplitude was considered as abnormal if it was below the $5^{\text {th }}$ percentile, for implicit times if it was above the $95^{\text {th }}$ percentile. LA $0.3-10.0 \mathrm{cds} / \mathrm{m}^{2}$ flash strengths were used for the single flash cone response. The LA $3.0 \mathrm{cds} / \mathrm{m}^{2}$ for the $30 \mathrm{~Hz}$ flicker response (cone response). DA $0.01 \mathrm{cds} / \mathrm{m}^{2}$ for the single flash rod response and DA $3.0-30.0 \mathrm{cds} / \mathrm{m}^{2}$ for the single flash combined rod/ cone response. In order to be defined abnormal, the same type of abnormality needed to be present in at least two consecutive flash intensities, except for the rod ERG, were the DA $0.01 \mathrm{cds} / \mathrm{m}^{2}$ needed to be abnormal. Abbreviations: $E R G=$ electroretinography, $I Q R=$ interquartile range, $L A=$ light adapted, $D A=$ dark adapted. 


\begin{tabular}{|c|c|c|c|c|c|}
\hline & & $\begin{array}{l}\text { Cut off } \\
\text { value }\end{array}$ & $\begin{array}{c}\mathrm{HC} \\
\text { median }[\mathrm{IQR}]\end{array}$ & $\begin{array}{c}\text { Uveitis } \\
\text { median [IQR] }\end{array}$ & P-value \\
\hline & A-wave impli & & & & \\
\hline & 0.0001 & & $80.49[76.15,85.31]$ & $80.00[75.12,82.38]$ & 0.094 \\
\hline & 0.0003 & & $74.19[69.88,76.96]$ & $75.00[71.00,79.00]$ & 0.015 \\
\hline & 0.001 & & $61.21[58.33,64.40]$ & $63.00[60.00,66.50]$ & $<0.001$ \\
\hline & 0.003 & & $49.44[46.76,52.50]$ & $52.00[49.00,55.00]$ & $<0.001$ \\
\hline & 0.01 & & $39.17[37.42,40.67]$ & $41.50[39.50,44.00]$ & $<0.001$ \\
\hline & 0.03 & & $32.75[31.35,34.25]$ & $34.50[33.00,37.00]$ & $<0.001$ \\
\hline & 0.1 & & $28.35[27.25,29.25]$ & $30.00[28.50,32.00]$ & $<0.001$ \\
\hline & 0.3 & & $25.57[24.80,26.29]$ & $27.00[26.50,29.00]$ & $<0.001$ \\
\hline & 1.0 & & $23.21[22.54,23.88]$ & $24.50[24.00,26.00]$ & $<0.001$ \\
\hline & 3.0 & 17.70 & $16.08[15.44,16.68]$ & $17.50[16.50,18.00]$ & $<0.001$ \\
\hline & 10.0 & 15.53 & $13.21[12.24,14.07]$ & $15.00[14.00,16.00]$ & $<0.001$ \\
\hline & 30.0 & 14.78 & $12.18[11.01,13.29]$ & $13.00[11.50,14.00]$ & $<0.001$ \\
\hline & B-wave ampl & & & & \\
\hline & 0.0001 & & $21.66[15.12,33.48]$ & $16.32[11.42,24.06]$ & $<0.001$ \\
\hline & 0.0003 & & $62.56[42.33,83.97]$ & $32.88[19.95,52.29]$ & $<0.001$ \\
\hline & 0.001 & & $131.65[100.48,170.25]$ & $79.68[51.60,118.15]$ & $<0.001$ \\
\hline & 0.003 & & $210.20[170.83,271.10]$ & $147.90[104.27,203.80]$ & $<0.001$ \\
\hline$\frac{\frac{\omega}{2}}{0}$ & 0.01 & 169.14 & $282.30[229.67,349.78]$ & $226.30[164.90,281.50]$ & $<0.001$ \\
\hline 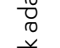 & 0.03 & & $309.30[252.05,376.98]$ & $265.95[206.80,322.88]$ & $<0.001$ \\
\hline 竞 & 0.1 & & $309.10[253.65,382.10]$ & $270.50[210.85,329.75]$ & $<0.001$ \\
\hline & 0.3 & & $349.15[284.58,418.35]$ & $299.30[229.90,359.00]$ & $<0.001$ \\
\hline & 1.0 & & $384.50[318.62,475.15]$ & $338.70[267.75,414.25]$ & $<0.001$ \\
\hline & 3.0 & 260.52 & $401.05[333.13,485.07]$ & $350.00[276.65,413.45]$ & $<0.001$ \\
\hline & 10.0 & 286.91 & $414.50[353.75,507.90]$ & $380.40[299.20,453.45]$ & $<0.001$ \\
\hline & 30.0 & 282.89 & $418.75[344.52,507.90]$ & $379.20[295.95,448.70]$ & $<0.001$ \\
\hline & B-wave impli & & & & \\
\hline & 0.0001 & & $131.75[124.99,141.04]$ & $125.50[112.00,134.00]$ & $<0.001$ \\
\hline & 0.0003 & & $135.51[130.78,140.82]$ & $134.00[127.50,139.50]$ & 0.027 \\
\hline & 0.001 & & $126.64[120.23,132.19]$ & $127.00[121.00,132.50]$ & 0.395 \\
\hline & 0.003 & & $110.52[105.14,116.79]$ & $112.00[106.00,119.00]$ & 0.096 \\
\hline & 0.01 & 104.30 & $89.20[84.38,96.42]$ & $93.00[85.50,101.00]$ & $<0.001$ \\
\hline & 0.03 & & $75.88[70.10,82.97]$ & $78.00[73.00,83.38]$ & $<0.001$ \\
\hline & 0.1 & & $58.65[53.65,67.33]$ & $63.00[57.50,71.50]$ & $<0.001$ \\
\hline & 0.3 & & $49.56[47.30,52.83]$ & $53.00[50.50,56.75]$ & $<0.001$ \\
\hline & 1.0 & & $49.45[47.11,52.74]$ & $50.50[48.50,54.50]$ & $<0.001$ \\
\hline & 3.0 & 58.09 & $51.77[49.78,54.25]$ & $55.50[52.50,57.00]$ & $<0.001$ \\
\hline & 10.0 & 56.05 & $51.52[49.22,54.18]$ & $55.00[53.50,56.00]$ & $<0.001$ \\
\hline & 30.0 & 55.14 & $49.32[43.50,52.08]$ & $52.50[51.00,54.00]$ & $<0.001$ \\
\hline
\end{tabular}


Chapter 2
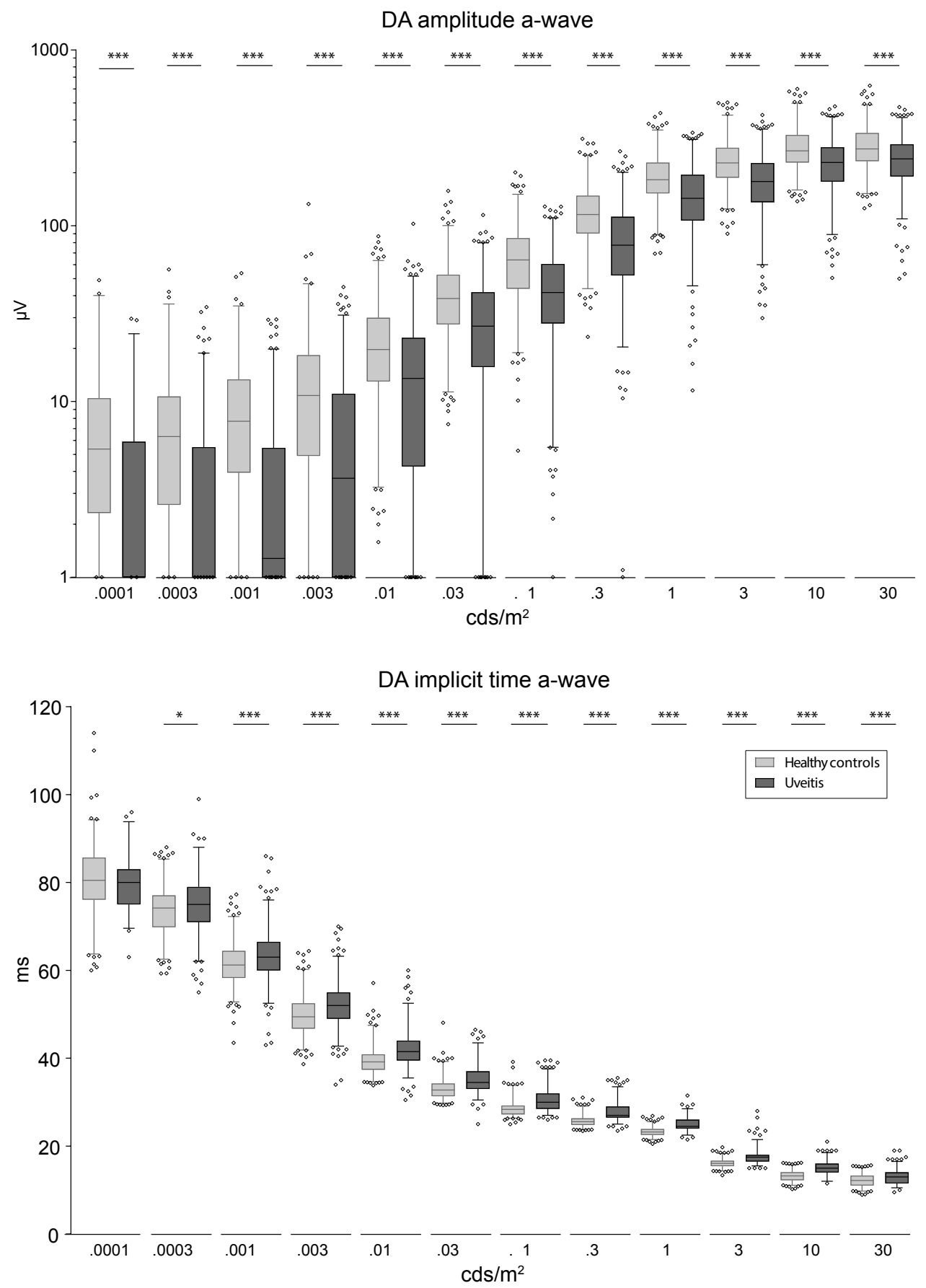

Supplemental figure 1: Boxplots of the amplitudes and implicit times of different flash intensities of the /single flash) dark-adapted a-wave of uveitis patients compared to healthy controls. Statistical testing was performed using Mann-Whitney-U test. " $P$-value $<0.05$. ${ }^{* * * *} P$-value of $<0.001$. Abbreviations: $c d s / \mathrm{m}^{2}=$ candela . seconds $/$ squared meters, $D A=$ dark adapted,$m s=$ milliseconds, $\mu V=$ microvolts. 
Prolonged cone b-wave is associated with severity of inflammation

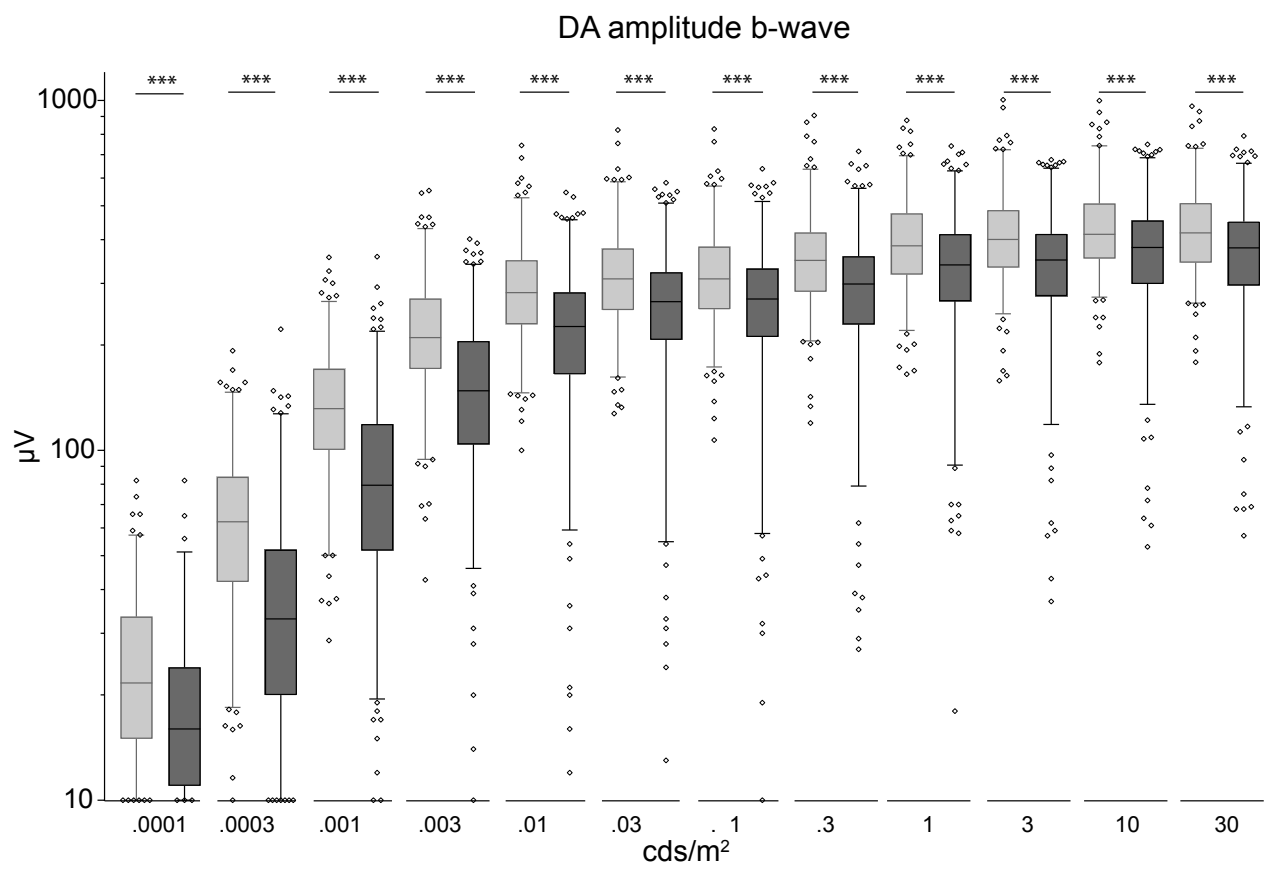

DA implicit time b-wave

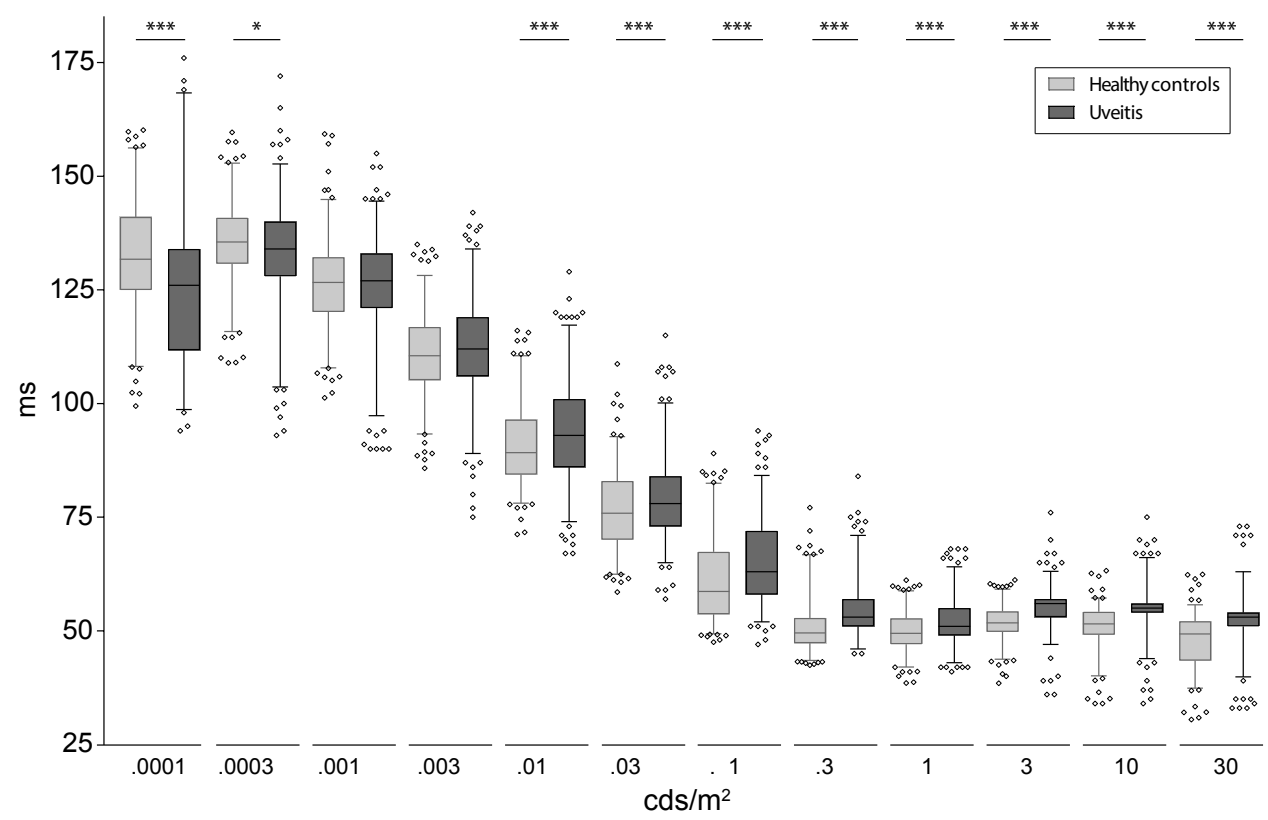

Supplemental figure 2: Boxplots of the amplitudes and implicit times of different flash intensities of the single flash dark-adapted b-wave of uveitis patients compared to healthy controls. Statistical testing was performed using Mann-Whitney-U test. "P-value $<0.05$. ${ }^{* * *} P$-value of $<0.001$. Abbreviations: $\mathrm{cds} / \mathrm{m}^{2}=$ candela . seconds $/$ squared meters, $D A=$ dark adapted, $m s=$ milliseconds, $\mu V=$ microvolts. 
LA amplitude a-wave

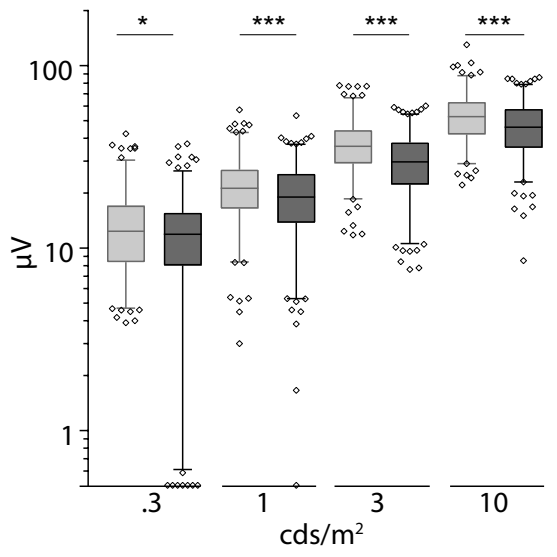

$\square$ Healthy controls

Uveitis

LA implicit time a-wave

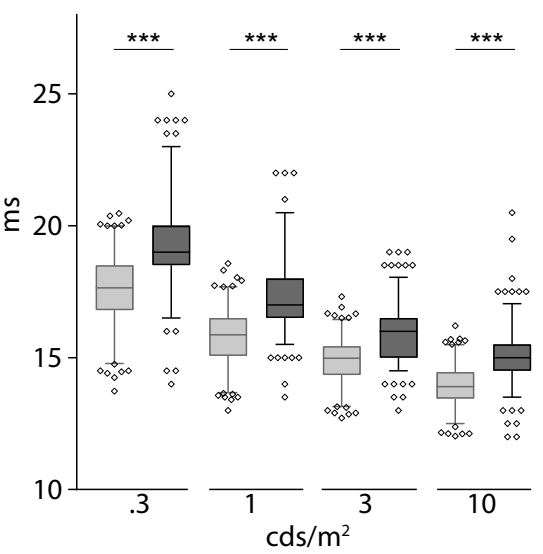

LA amplitude b-wave

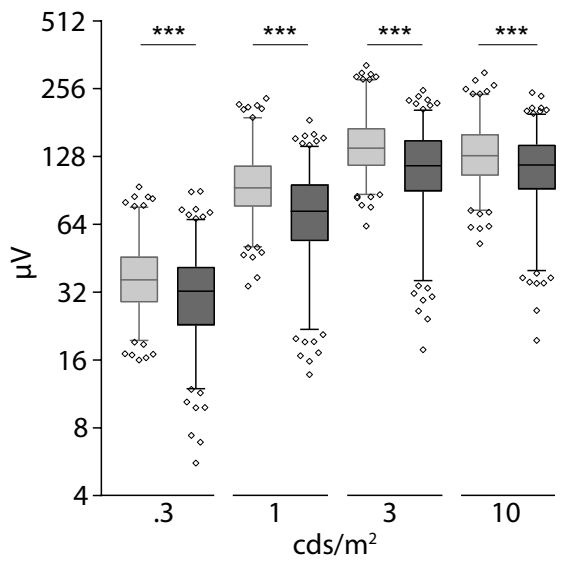

$30 \mathrm{~Hz}$

Amplitude

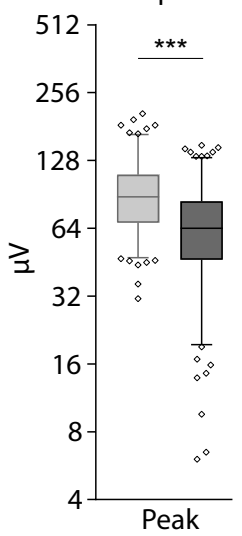

$30 \mathrm{~Hz}$

Implicit time

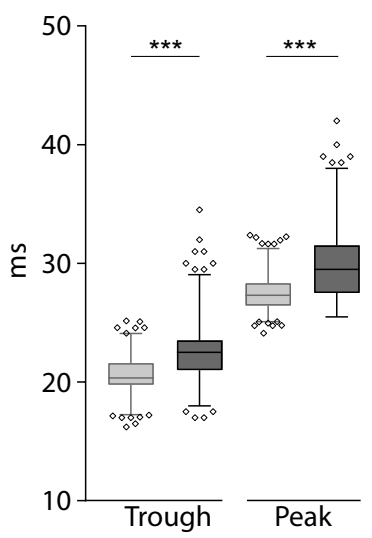

Supplemental figure 3: Boxplots of the light adapted a-wave (implicit times and amplitudes). B-wave (amplitudes) of different flash intensities and the $30 \mathrm{~Hz}$ flicker response of uveitis patients compared to healthy controls. Statistical testing was performed using Mann-Whitney- $U$ test. ${ }^{* * * *} P$-value of < 0.001 . Abbreviations: $\mathrm{cds} / \mathrm{m}^{2}=$ candela $\cdot$ seconds $/$ squared meters, $L A=$ light adapted, $\mathrm{ms}=$ milliseconds, $\mu \mathrm{V}=$ microvolts. 
Prolonged cone b-wave is associated with severity of inflammation
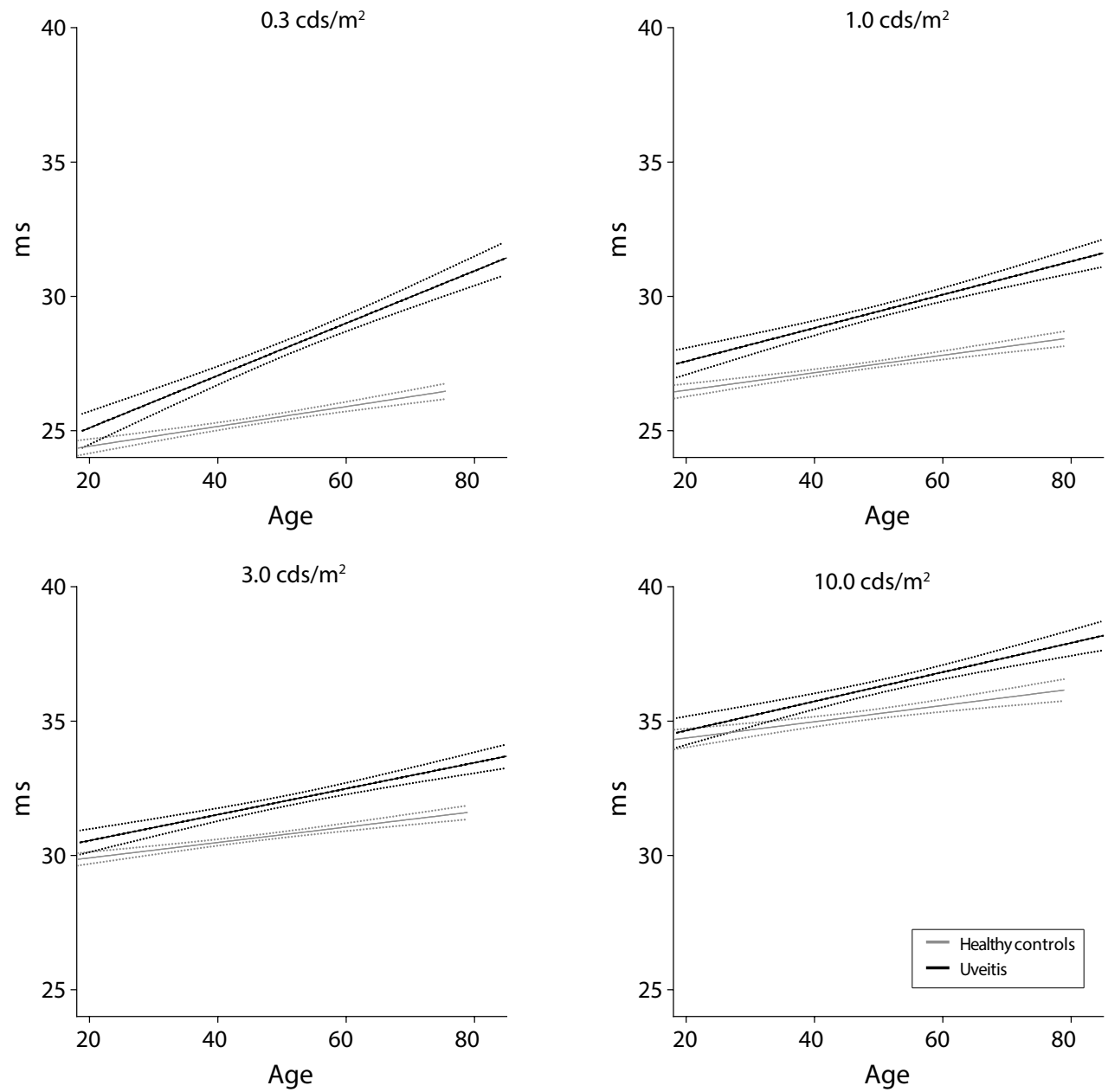

Supplemental figure 4: Logistic regression of the effect of age on the implicit time of the single flash cone $b$-wave (shown in ms on the $y$-axis) of uveitis patients compared to healthy controls. Abbreviations: $c d s / \mathrm{m}^{2}=$ candela $\cdot$ seconds $/$ squared meters, $\mathrm{ms}=$ milliseconds. 
Chapter 2
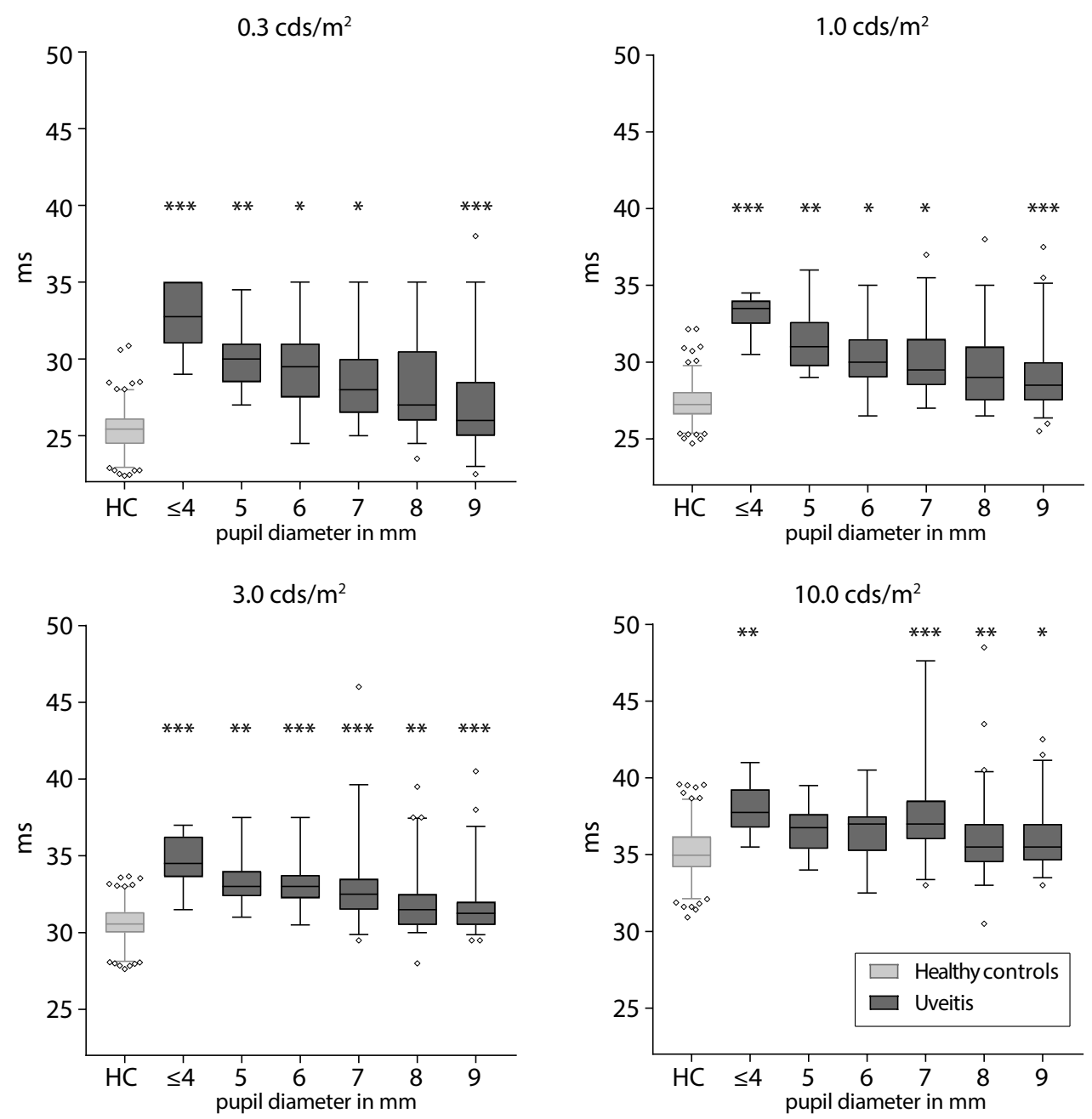

Supplemental figure 5: Effect of different pupil sizes on the implicit time of the single flash cone b-wave (shown in ms on the $y$-axis) of uveitis patients compared to healthy controls (HC) with fully dilated pupils. Statistical testing was performed using Mann-Whitney-U test. ${ }^{*} P$-value $<0.05$. ${ }^{*} P$-value $<0.01$. ${ }^{* * * *} P$-value of $<0.001$. Abbreviations: $\mathrm{cds} / \mathrm{m}^{2}=$ candela $\cdot$ seconds $/$ squared meters, $\mathrm{ms}=$ milliseconds. 
Prolonged cone b-wave is associated with severity of inflammation 


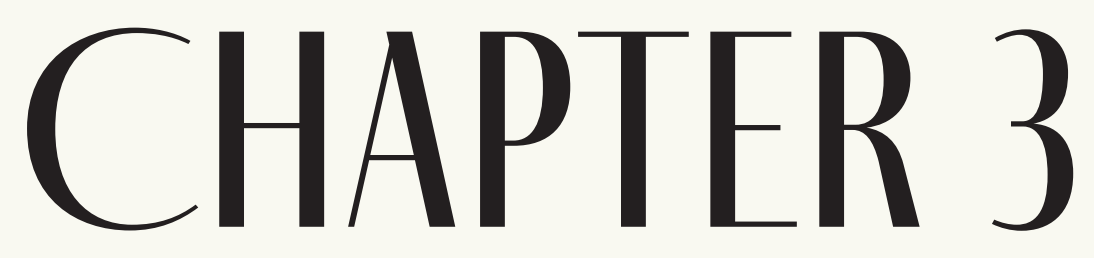

\section{ELECTRORETINOGRAM ABNORMALITIES IN NON-INFECTIOUS UVEITIS OFTEN PERSIST}

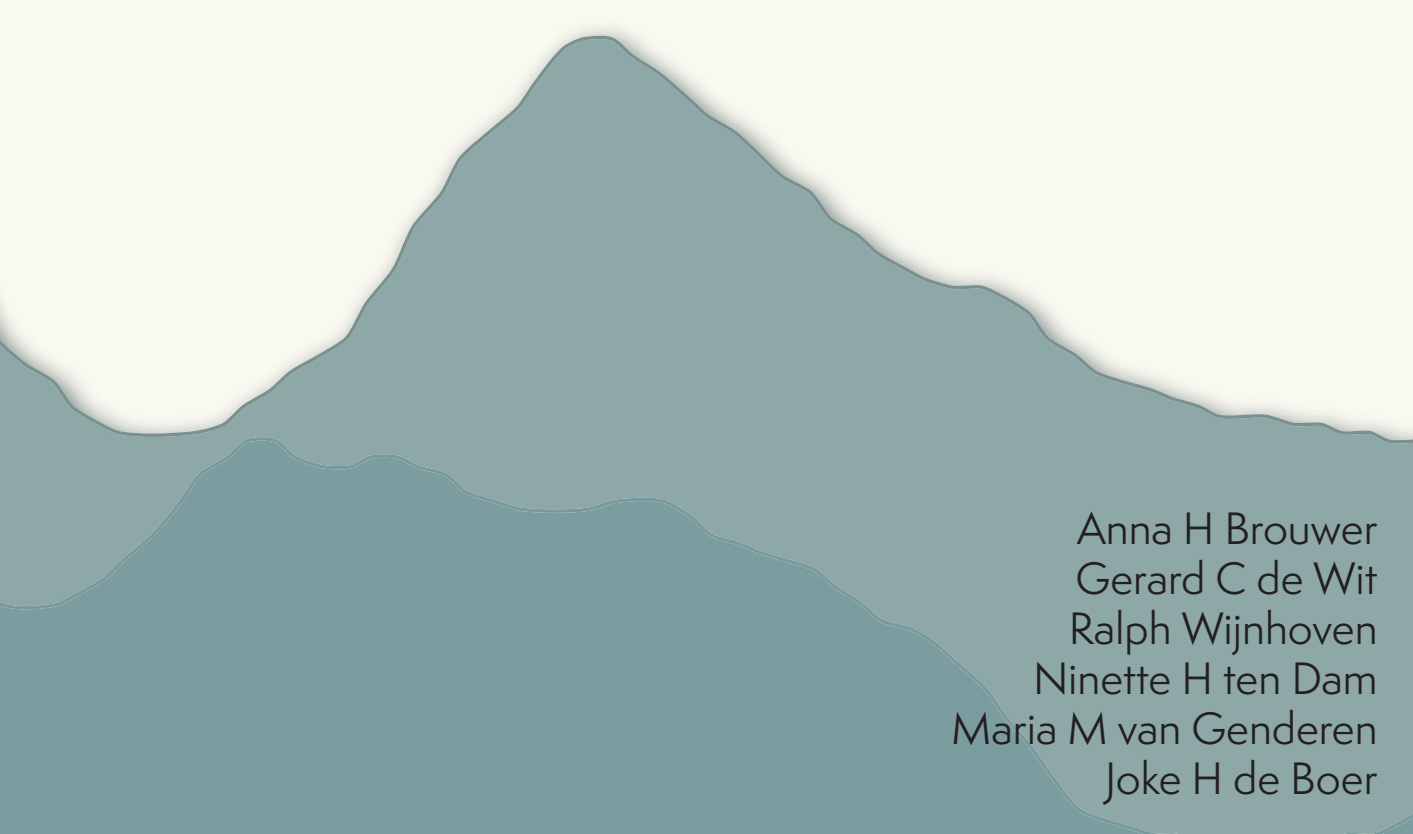


Chapter 3

\section{ABSTRACT}

\section{Purpose}

In uveitis, a prolonged implicit time of the cone b-wave is a characteristic electroretinogram (ERG) abnormality. We investigated whether this can improve or deteriorate over time and which clinical factors are associated with change.

\section{Methods}

Prospective cohort study. Patients with a non-infectious uveitis were included. An ERG was measured in the first year of uveitis onset and a follow-up ERG one year later. Changes in the implicit time of the cone b-wave were investigated in relation to clinical parameters including the following: demographics, uveitis characteristics, treatment, best corrected visual acuity, optical coherence tomography parameters and fluorescein angiography scores.

\section{Results}

Of 98 eyes (63 patients), 40 showed a prolonged cone b-wave on the first ERG, which improved in 10 eyes. Eyes with an improved ERG more often had a panuveitis with initially a higher incidence of cells in the anterior chamber during the first ERG, which resolved at the time of their follow-up ERG. Five of the 58 eyes with a normal first ERG, had a deteriorated follow-up ERG. These eyes had more frequently an active uveitis at the time of the follow-up ERG. Of the 78 eyes with a stable cone b-wave, 16 had a quiescent inflammation during follow-up. There were no differences in age or treatment.

\section{Conclusion}

In most patients with non-infectious uveitis, ERG abnormalities appear to be irreversible, even when the inflammation becomes quiescent. However, some ERGs improved, which was associated with reduction in inflammation of the anterior chamber due to panuveitis. In contrast a worsened ERG was associated with a persistence of inflammation. 
ERG abnormalities in non-infectious uveitis often persist

\section{INTRODUCTION}

Uveitis comprises of a group of multiple diseases with an inflammation of the uvea and its adjacent structures, including the retina.' If this inflammation is severe, it can be treated with immune-modulating drugs. However, despite intensive treatment strategies with these medicines, vision loss can still develop. ${ }^{2}$ This vision loss can be the result of several complications of intraocular inflammation.

Well-known complications are macular oedema, cataract and glaucoma. ${ }^{3}$ However, retinal atrophy and loss of retinal function may also occur. Recently, we described a characteristic type of ERG abnormality in uveitis: a prolonged cone b-wave, which was seen in all anatomic subtypes including anterior uveitis. This prolonged cone b-wave was associated with a more severe inflammation of both present and past. ${ }^{4}$

So far it is unknown to what extent ERG abnormalities in uveitis are reversible. We found that the prolonged cone b-wave could still be present in eyes in which inflammation had become inactive at the time of ERG recording. However, there are reports of ERG abnormalities that improve in uveitis. For instance, in birdshot chorioretinitis (BSCR) the ERG can normalize after treatment.5.6 On the other hand, in Behçet disease, the ERG does not improve, even when the inflammation becomes quiet. ' In other uveitis entities, such as in AMPPE, MEWDS and AZOOR, the ERG may either improve or deteriorate over time..$^{8-10}$

To gain more insights into factors that may contribute to either an improvement, or a worsening of retinal function in uveitis patients, we investigated changes in the ERG over time in relation to clinical factors such as anatomical localization and diagnosis, severity of inflammation and treatment.

\section{$\underline{\text { METHODS }}$}

\section{Design and patient population}

This is a one-year follow-up study of a prospective cohort study which describes the retinal function of uveitis patients by using the ERG. ${ }^{4}$ Inclusion criteria for this follow-up study were as follows: a non-infectious uveitis and a disease duration of less than 1 year at the time of inclusion for the previous study. The results of the first ERGs were part of a larger cohort study which was previously described. ${ }^{4}$ The diagnoses which were included are as follows: Behçet disease, birdshot, chorioretinitis, human leukocyte antigen (HLA)-B27 associated uveitis, Vogt-Koyanagi-Harada disease, sarcoidosis, and uveitis of unknown cause. 
We included 72 patients (114 uveitis eyes) for this follow-up study. Of the initial 80 patients who had an ERG in the first year of onset of uveitis, four no longer wanted to participate, three were referred back to another hospital and one moved to another country.

All patients were $\geq 18$ years of age, mentally competent, and gave informed consent to participate. Patients were seen at the University Medical Centre Utrecht, a tertiary referral centre for uveitis. Patients with juvenile idiopathic arthritis, diabetic retinopathy, retinal dystrophy, family history of retinal dystrophy, myopic degeneration, or severe media opacities were excluded. Ethical approval was requested and obtained from the Medical Ethical Research Committee of the University Medical Centre Utrecht. This study was conducted in compliance with the ethical principles of the Declaration of Helsinki.

\section{ERG analysis}

Electroretinograms were performed on the same day as an outpatient clinic visit when uveitis activity was assessed. Electroretinograms were measured according to an extended protocol, with more flash strengths than the standard International Society for Clinical Electrophysiology of Vision (ISCEV) protocol." The flash strengths increase with approximately $0.5 \mathrm{log}$ units steps and range from 0.0001 to $30.0 \mathrm{cds} / \mathrm{m}^{2}(12$ flash strengths) for the dark-adapted ERG (DA) and from 0.3 to $10.0 \mathrm{cds} / \mathrm{m}^{2}$ (4 flash strengths) for the light-adapted ERG (LA) and include a $30 \mathrm{~Hz}$ flicker response as well.

We used Dawson-Trick-Litzkow (DTL) electrodes as active electrodes, and an Espion E3 system with ColorDome stimulator (Diagnosys LLC, Cambridge, UK) for flash stimulation. Full details of the ERG measurement procedure, as well as our reference values, were previously described. ${ }^{4}$

In this study we focus on changes in the implicit time of the cone b-wave, but other aspects of the ERG were investigated as well. The implicit time of the cone b-wave is correlated to the peak implicit time of the $30 \mathrm{~Hz}$ flicker response (Spearman's rho coefficient $0.620-0.814 p<.001) .{ }^{4}$ Because the prolonged cone b-wave was the most frequent and characteristic ERG abnormality in uveitis that we observed in our previous study, we focused on differences in the cone b-wave

To our knowledge there are no internationally accepted criteria to define whether an ERG has improved or worsened, but only criteria to describe whether an ERG is normal or abnormal (in- or outside reference values). In this study we used the difference in milliseconds ( $\mathrm{ms}$ ) of the implicit time of the cone b-wave of first ERG minus the follow-up ERG to define whether the ERG was stable, improved or worsened. Because pupil size affects the implicit time of the cone $b$ wave, we excluded eyes with a pupil size difference $>1 \mathrm{~mm}$ between the first and follow-up ERG. 
To determine how many ms the implicit time needed for change to be defined as significantly changed, we used data of a previous study where we measured a LA ERG twice in 200 uveitis patients, to assess the effects of DTL position on the ERG. ${ }^{12}$ The DTL position had an effect on amplitudes, but not on implicit times of the ERG. Because this study was in essence a repeated measurement study, we used the difference in implicit time between the two ERGs of each eye to determine a normal repeatability distribution. We defined improvement as a reduction in cone b- wave implicit time of $\geq 2$ SD of this distribution $\left(2.7,1.7,1.6\right.$ and $1.3 \mathrm{~ms}$ for the $0.3,1.0,3.0$ and $10.0 \mathrm{cds} / \mathrm{m}^{2}$ flashes respectively) in at least two consecutive flash strengths. We defined worsening as an increase in implicit time with $\geq 2$ SD in at least two consecutive flash strengths. We defined the ERG as stable if the implicit time of the first and second ERG was within $95 \%$ of the repeatability distribution $(<2$ SD). Figure 1 shows examples of a patient with a worsened implicit time $(A)$ and of a patient with an improved implicit time of the cone b-wave (B).

Because the focus of this manuscript is on the implicit time of the cone b-wave of the ERG, the terms stable, improved, or worsened ERG mean a stable, improved, or worsened implicit time of the cone b-wave, respectively.

\section{Clinical parameters}

Medical records were reviewed for age, gender, medical history, uveitis diagnosis, and anatomical localization of uveitis. On each of the two outpatient clinical visits we recorded for each eye the BCVA and graded uveitis activity according to the SUN criteria. ${ }^{13}$ We also noted the presence of possible media opacities and other factors which might influence the ERG such as: posterior synechiae, corneal clarity, lens clarity, vitreous haze, and pupil size.

Also, we recorded per patient if they were treated at the time of ERG recording with systemic steroids, disease modifying anti-rheumatic drugs (DMARDS) (i.e. methotrexate (MTX), azathioprine, mycophenolate mofetil, mycophenolate sodium, or cyclosporine) or biologicals (adalimumab or infliximab).

We scored fluorescein angiograms (FA) which were performed within 3 months to the time of ERG recordings as an indication of the severity of inflammation. FAs were scored by an experienced ophthalmologist (JdB) who was blinded regarding the ERG results, using the Fluorescein angiographic scoring system of the Angiography Scoring for Uveitis Working Group (ASUWOG).14 This FA scoring system scores individual aspects of an FA, which are added up to a final FA score. This FA score, which is the summation of each of these individual sub-scores helps to quantify the magnitude of retinal inflammation. In our previous study, eyes with a higher FA score 
more frequently had a prolonged cone b-wave. ${ }^{4}$ If FAs were performed on the same day as the ERG, there was at least an hour between the FA and ERG. ${ }^{15}$

To determine whether cystoid macula oedema (CMO) was present, we used optical coherence tomography (OCT) (Zeiss, Cirrus HD OCT 5000) scans of the same day as the ERG. We defined an active uveitis as the presence of cells in the anterior chamber and/or a vitritis of $\geq 1+$ cells and/or a fluorescein angiograms (FA) score of $>1$.

\section{Statistical analysis}

For statistical analysis R version 1.1.456 (C 2009 - 2018 RStudio, Inc.) was used. ERG changes between the first and the follow-up ERG were investigated using the paired t-test or Wilcoxon paired sample test, depending on the distribution of data. Data distribution was evaluated with qq-plots, histograms and Shapiro Wilk test. All significances were two tailed. We considered $p$-values $<0.05$ as statistically significant.

Next, we investigated whether eyes that had a prolonged cone b-wave during the first ERG could improve, or worsen even more in the follow-up ERG. Also, we investigated whether eyes that had an implicit time that was within our reference values, could worsen in the follow-up ERG. These outcomes were then investigated in relation to clinical parameters using Pearson chi-square test or Fischer's exact test for categorical variables, and a Student t-test or a Mann-Whitney- $U$ test for continuous variables, depending on the distribution of data.

For patient specific characteristics such as age, one eye per patient was analysed. For patients with a bilateral uveitis where both eyes had the same cone b-wave outcome (i.e. both uveitis eyes stable, improved, and worsened), one eye was selected at random. If the cone b-wave outcome differed between uveitis eyes of the same patients, the eye with the changed cone b-wave outcome (i.e. improved or worsened) was selected for analysis of patient-specific variables such as age or diagnosis. None of the patients had one eye which improved and one that worsened.

\section{$\underline{\text { RESULTS }}$}

\section{ERG results}

Sixteen eyes of nine patients were excluded from further analysis, because the pupil size difference was $>1 \mathrm{~mm}$ between the two ERGs and a disparity in pupil size can cause a change in b-wave implicit time that is independent of retinal pathology. Of these excluded eyes, 2 eyes showed an improvement, 1 eye worsened and 13 eyes had no significant difference in the implicit time of the cone b-wave. 
On a group level, the implicit times of the cone b-wave were not statistically significantly different between the first and the follow-up ERG (see Table 1). The ERG changed significantly in 20 eyes: in 10 eyes the implicit time improved and in 10 eyes it worsened, based on our cut-off values (see Figure 1 for examples).

There was no predominant flash strength at which this change in implicit time was most prominent. In other ERG parameters (see Supplemental table 1), there were also no consistent significant changes between the first and the follow-up ERG. There was a significant correlation between the peak implicit time of the $30 \mathrm{~Hz}$ flicker response and a prolonged cone b-wave of both the first (Spearman's rho $=0.739, p<$ 0.001) and follow-up ERG (Spearman's rho $=0.695, p<0.001$ )

Table 1: Results of implicit time of the cone b-wave of the first and follow-up electroretinogram

\begin{tabular}{|c|c|c|c|c|}
\hline \multicolumn{2}{|c|}{$\begin{array}{l}\text { Implicit time cone b-wave, } \\
\text { median [IQR] }\end{array}$} & \multirow{2}{*}{$\begin{array}{c}\begin{array}{c}1^{\text {st }} \text { ERG } \\
\text { (<1 year of diagnosis) }\end{array} \\
27.00[25.50,30.50]\end{array}$} & \multirow{2}{*}{$\frac{\begin{array}{c}\text { Follow-up ERG } \\
(<1 \text { year later })\end{array}}{27.00[26.00,30.38]}$} & \multirow{2}{*}{$\frac{\text { P-value }}{0.544}$} \\
\hline $\mathrm{cds} / \mathrm{m}^{2}$ & 0.3 & & & \\
\hline & 1.0 & $29.25[28.00,31.00]$ & $29.25[28.00,31.00]$ & 0.960 \\
\hline & 3.0 & $31.50[30.62,33.50]$ & $32.00[31.00,33.50]$ & 0.760 \\
\hline & 10.0 & $35.50[35.00,37.50]$ & $35.75[35.00,37.00]$ & 0.510 \\
\hline
\end{tabular}

Results of the first and follow-up ERG of the different flash strengths $\left(\mathrm{cds} / \mathrm{m}^{2}\right)$ of the implicit time of the cone $b$-wave of 98 uveitis eyes. Eyes with a pupil size difference $>1 \mathrm{~mm}$ between the first and the follow-up ERG were excluded. Results of the other ERG parameters are shown in supplementary table 1. P-values were tested with the paired Wilcoxon signed rank test (no normal distribution of data). All significances were two-tailed. Abbreviations: $E R G=$ electroretinogram, $I Q R=$ interquartile range, $\mathrm{cds} / \mathrm{m}^{2}=$ candela $\cdot$ seconds $/$ squared meters.

\section{ERG changes in relation to clinical parameters}

Table 2 shows possible associations between changes in the ERG and patient characteristics as well as treatment. Regarding age, gender, anatomical localization, and uveitis diagnosis there were no differences in changes in the implicit time of the cone b-wave.

We also observed no statistically significant differences in treatment between patients with a different ERG outcome. However, more patients that had an improved follow-up ERG were treated with systemic medication after the first ERG. In contrast, one of the patients with a worsened ERG ceased systemic medication between the first and follow-up ERG.

There was no significant association between the duration of uveitis and start of systemic medication with either an improvement or a worsening of the ERG. However, patients with an abnormal first ERG, started systemic medication later compared to 

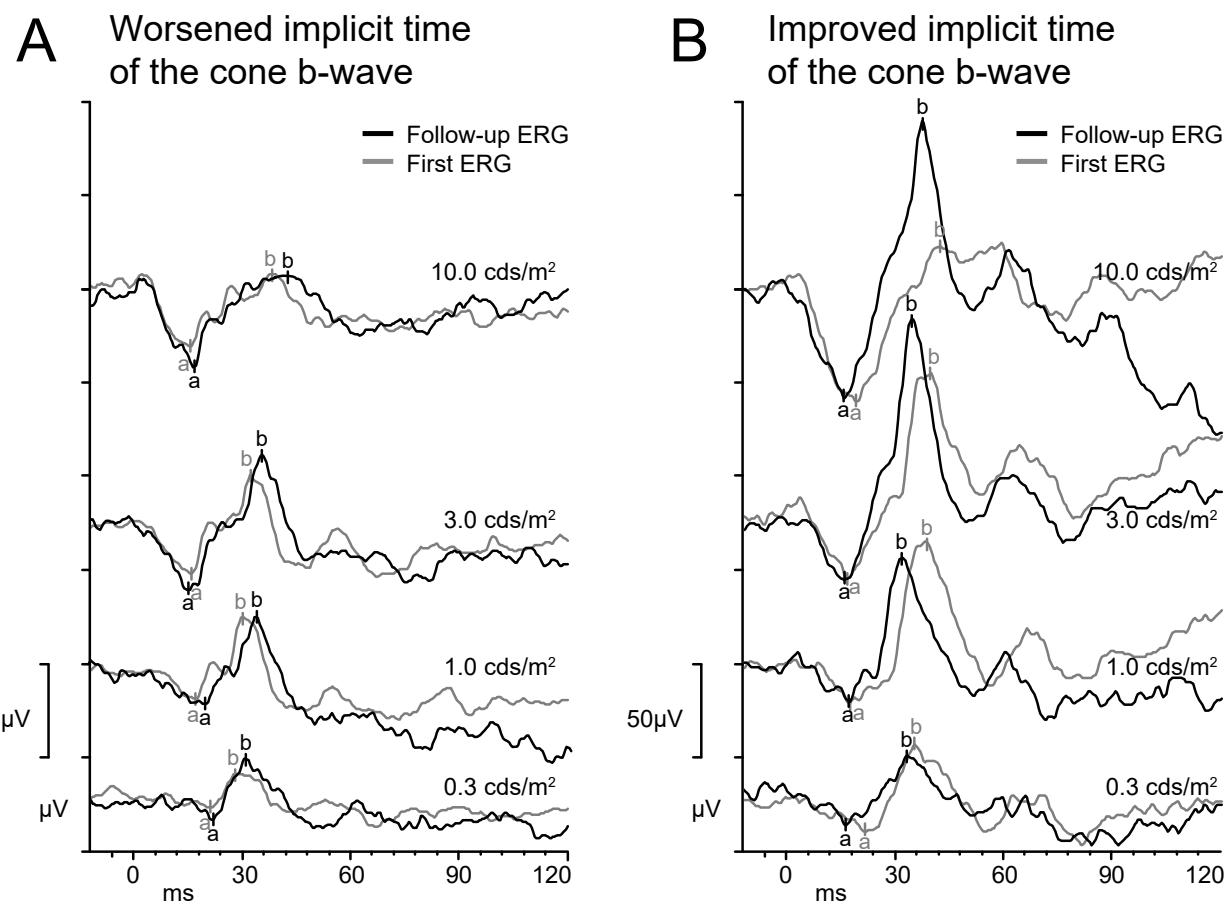

Figure 1: Examples of a patient with a worsened (A) implicit time of the cone $b$-wave, and of a patient with an improved implicit time of the cone b-wave (B). The first ERG is printed in grey and the follow-up ERG a year later in black.

patients with a normal first ERG (median uveitis duration on months of 1.2 versus 2.9, respectively, $p=0.382$ ). This difference was not significant and overall patients with an abnormal first ERG were treated more often with systemic medication. Also, we observed no differences based on uveitis diagnosis.

Table 3 shows possible associations for an improved implicit time of the cone b-wave and uveitis characteristics. Eyes with an improved implicit time more often had a panuveitis with cells in the anterior chamber during the first ERG. This inflammation in the anterior chamber was resolved during the second ERG in all improved eyes.

Table 4 shows possible associations between a worsening of the implicit time of the cone b-wave in eyes with an initial normal ERG and uveitis characteristics. Eyes that had a worsened ERG, more frequently had an active uveitis during the second ERG. This activity was mostly due to a vitritis, which was often not present during the first ERG. Besides vitritis, BCVA of these eyes was on average also slightly worse during the first ERG, but improved during the follow-up ERG.

Eyes with an improved ERG had more frequently media opacities and/or a small pupil size during the first ERG, whereas eyes with a worsened ERG had these findings more frequently during the second ERG. 
Table 2. Patient characteristics of uveitis patients with different outcomes of the implicit time of the cone b-wave

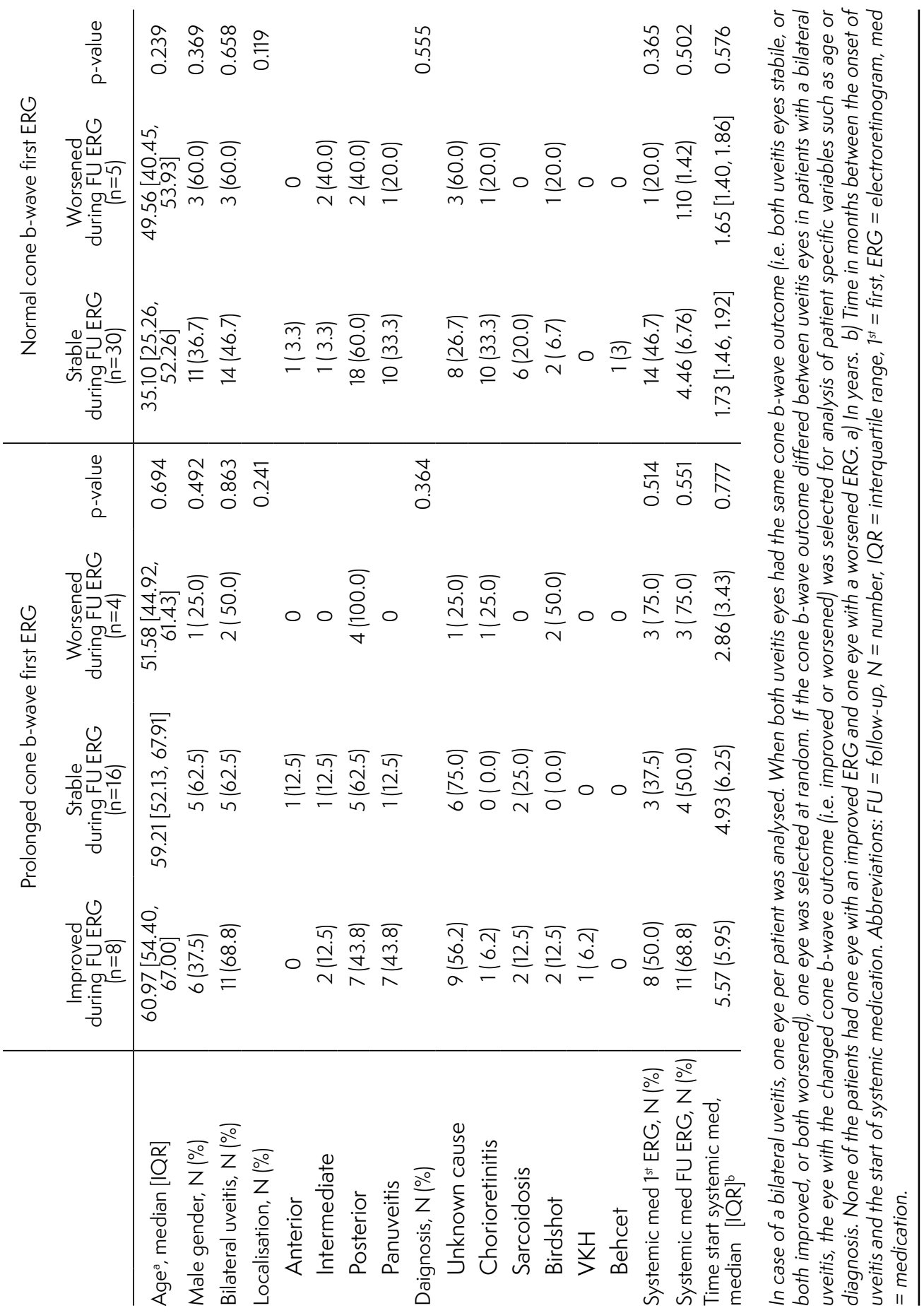


Table 3. Uveitis activity and vision of eyes (40) with a prolonged cone b-wave during their first electroretinogram

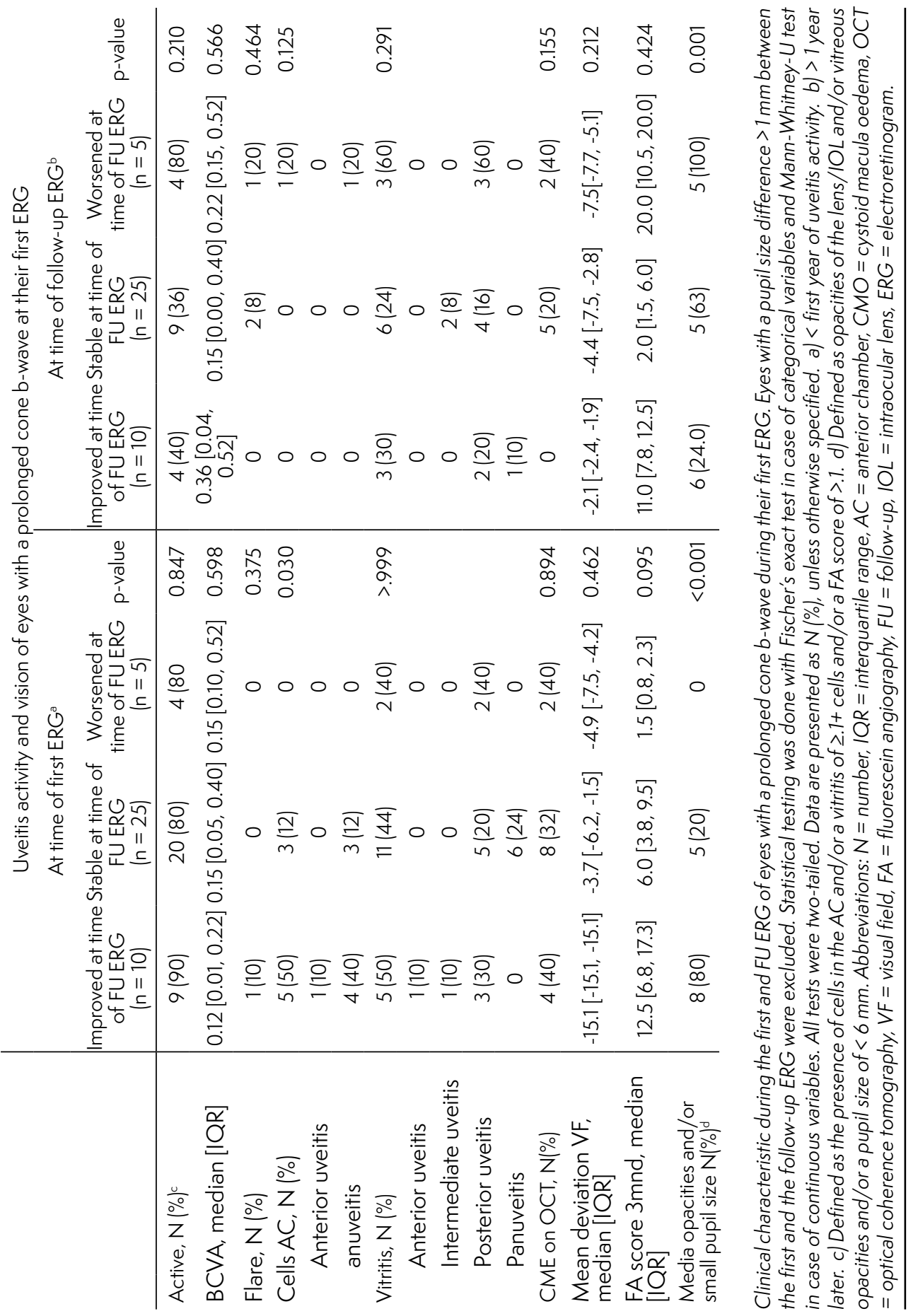


Table 4. Uveitis activity and vision of eyes (58) with a normal implicit time of the cone $b$-wave during their first electroretinogram

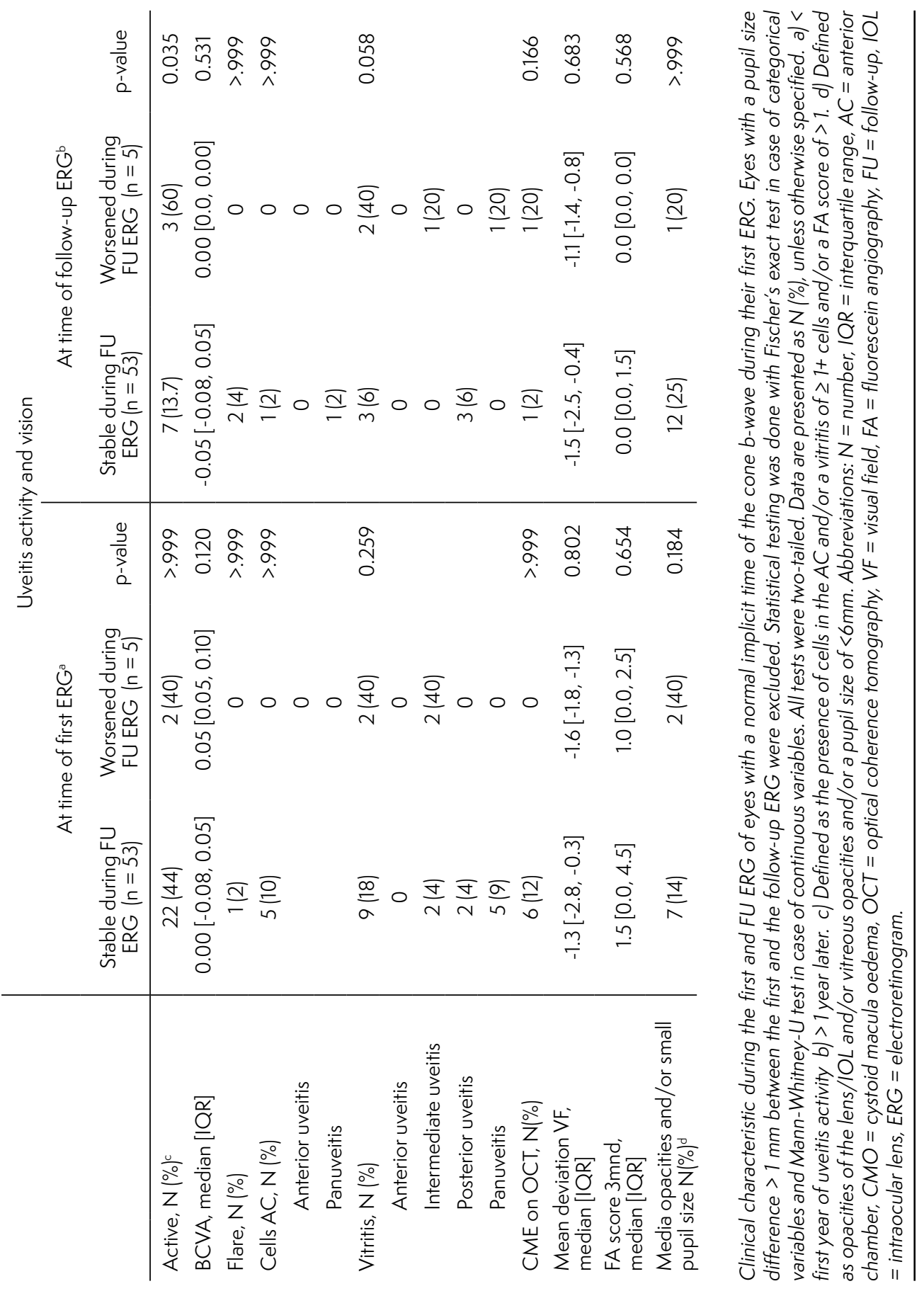


It is important to note that most of the eyes did not show significant ERG changes. In 78 eyes $(80 \%)$ the implicit time was stable, of which 25 eyes had a prolonged implicit time. Almost two third of these ( 16 eyes) had no signs of an active inflammation during the follow-up ERG.

Supplemental tables 2 and 3 give an overview of the different aspects of the FA score. There were no differences in these individually scored aspects of the FA between eyes with a different ERG outcome.

Supplemental table 4 shows the correlation matrix between statistically significant clinical factors, duration of uveitis, age, and treatment. Although many factors are statistically significant, all of the associations are weak (rho $<0.5$ ).

\section{DISCUSSION}

This study highlights that a prolongation of the implicit time of the cone b-wave occurs early on in the non-infectious uveitis and often persists. Only in a minority of cases, it can improve or deteriorate in the course of the disease. Improvement was mostly seen in eyes in which the inflammation became more quiescent, whereas a worsening was seen in eyes with a persistent inflammation. The delayed cone b-wave is probably due to an impaired photo-transmission to the bipolar cells. It remains speculative if this ERG abnormality is the first sign of evolving photoreceptor damage.

Although these findings indicate that it is important to treat the inflammation in uveitis adequately and early, we found no differences between the groups based on type of treatment. This might be due to several factors including variation between treatment strategies, as well as the effects of responders versus non-responders.

In eyes with an improved ERG, the inflammation in the anterior chamber had become quiescent. The majority of these patients had a panuveitis. It is likely that the improvement of the inflammation in the anterior chamber was associated with an improvement of the posterior segment, which should be the subject of further studies.

In most eyes the ERG abnormalities were irreversible, even without signs of active inflammation at the time of the follow-up ERG. Because we measured the first ERG in the first year of onset of disease, it indicates that the retinal damage can occur early on in the disease process. Patients with an abnormal first ERG started systemic treatment on average later than those with a normal first ERG. Furthermore, patients with an improved ERG were using systemic medication more often at the time of the follow-up ERG compared to the first ERG. Although these results showed a trend, the differences were not significant. One explanation could be that the patients in this study may represent a group with a more severe disease course, because this study was performed in a tertiary referral centre. 
Apart from the severity of inflammation, eyes with an improved ERG had more frequently media opacities or a small pupil size during their first ERG. In the lightadapted ERG implicit times increase when the intensity of the flash strength becomes stronger. Media opacities or a small pupil size can reduce the retinal illuminance of the stimulus flash leading to changes in b-wave implicit time of non-pathologic origin., ${ }^{412}$ Therefore, it is possible that the improved implicit time of the cone b-wave may even be underestimated due to the improvement of media opacities. Effects of changes in pupil size were addressed by excluding eyes with a pupil size difference $>1 \mathrm{~mm}$.

Because the implicit time of the cone b-wave in uveitis over time has not previously been studied, it is not possible to make comparisons with literature. However, there are some reports of follow-up ERG's in birdshot uveitis that show that the $30 \mathrm{~Hz}$ flicker response can improve after systemic treatment with corticosteroids, but not in all cases. ${ }^{5}$ Furthermore, other ERG parameters than the $30 \mathrm{~Hz}$ flicker response can be permanently affected in birdshot uveitis. ${ }^{16,17}$

In the current study, we could only replicate that the $30 \mathrm{~Hz}$ flicker response improved in the minority of the birdshot patients after the start of systemic treatment, possibly because 6 out of 7 of the included birdshot patients already used systemic medication at the time of the first ERG. In these patients the implicit time of the $30 \mathrm{~Hz}$ flicker response did improve in 4 out of 14 eyes, whereas the implicit time of the cone b-wave did not improve.

Some studies state that a change of $>30 \%$ in amplitude is necessary to define improvement or deterioration, based on Berson's study on decline in retinitis pigmentosa (RP). ${ }^{18}$ However, the ERG in RP is generally much more severely affected than in uveitis, where the amplitude often does not change significantly. Most studies that report on follow-up results of the ERG in uveitis, do not specify how improvement is defined, ,7-10 for instance, which aspect of the ERG changed during follow-up. In our study we observed that the different parameters of the ERG may behave quite differently. For uveitis monitoring, we found that the implicit time of the cone b-wave is the most sensitive parameter.

In summary, this study demonstrates that a prolonged implicit time of the cone b-wave often persists in uveitis. In a minority of cases, this loss of retinal function can improve if the inflammation becomes less active. This emphasizes the importance to treat the inflammation adequately and early in uveitis. Further research is needed to investigate to which extent the implicit time of the cone b-wave can be used as a prognostic marker in uveitis. 
Chapter 3

\section{ACKNOWLEDGEMENTS}

We would like to thank all patients for participating in the study. The authors were supported by the following foundations: Dr. F.P Fischer Stichting, and Bartiméus Fonds that contributed through UitZicht (the Netherlands). The funding organizations had no role in the design or conduct of this research. They provided unrestricted grants.

A.H. Brouwer, N.H. ten Dam, R. Wijnhoven and M.M. van Genderen have no financial disclosures. G.C. de Wit is employed as a medical physicist at Bartiméus, a low vision institute in the Netherlands. Furthermore, he is a sole proprietor, with his company Optical Diagnostics (http://www.opticaldiagnostics.com) he develops and sells ophthalmic software products and does sometimes consulting work. J.H. de Boer was payed by Abbvie for lectures.

We thank J. Ossewarde-van Norel and L. Ho (University Medical Centre Utrecht), for their help in recruiting patients to participate in this research, D. Gültzau, Y. Burgers and M. Ballast (University Medical Centre Utrecht) for their help in recording the ERGs, F. Riemslag and H. Talsma (Bartiméus Diagnostic Centre for complex visual disorders, Zeist) for giving additional electrophysiological advice and S. Risseeuw (University Medical Centre Utrecht) for her statistical advice. 
ERG abnormalities in non-infectious uveitis often persist

\section{$\underline{\text { REFERENCES }}$}

1. Thorne JE, Suhler E, Skup M, et al. Prevalence of Noninfectious Uveitis in the United States: A Claims-Based Analysis. JAMA Ophthalmol. 2016;134(11):1237-1245. doi:10.1001/jamaophthalmol.2016.3229

2. Suttorp-Schulten MS, Rothova A. The possible impact of uveitis in blindness: a literature survey. Br J Ophthalmol. 1996;80(9):844-848. http://www.ncbi.nlm.nih.gov/ pubmed/8962842.

3. Forooghian F, Yeh S, Faia LJ, Nussenblatt RB. Uveitic foveal atrophy: clinical features and associations. Arch Ophthalmol (Chicago, III 1960). 2009;127(2):179-186. doi:10.1001/archophthalmol.2008.564

4. Brouwer AH, de Wit GC, Ten Dam NH, Wijnhoven R, van Genderen MM, de Boer $\mathrm{JH}$. Prolonged cone b-wave on electroretinography is associated with severity of inflammation in non-infectious uveitis. Am J Ophthalmol. June 2019. doi:10.1016/j. ajo.2019.05.028

5. Holder GE, Robson AG, Pavesio C, Graham EM. Electrophysiological characterisation and monitoring in the management of birdshot chorioretinopathy. Br J Ophthalmol. 2005;89(6):709-718. doi:10.1136/bjo.2004.047837

6. Elbaz H, Besgen V, Rechberger K, Sekundo W, Apfelstedt-Sylla E. Electroretinogram and visual field changes in a case of birdshot chorioretinopathy. Doc Ophthalmol. 2017;134(2):149-153. doi:10.1007/s10633-017-9581-2

7. Hamza MME, Macky TA, Sidky MK, Ragab G, Soliman MM. INTRAVITREAL INFLIXIMAB IN REFRACTORY UVEITIS IN BEHCET'S DISEASE: A Safety and Efficacy Clinical Study. Retina. 2016;36(12):2399-2408. doi:10.1097/IAE.0000000000001109

8. Jacobson SG, Morales DS, Sun XK, et al. Pattern of retinal dysfunction in acute zonal occult outer retinopathy. Ophthalmology. 1995;102(8):1187-1198.

9. Sieving PA, Fishman GA, Jampol LM, Pugh D. Multiple evanescent white dot syndrome. II. Electrophysiology of the photoreceptors during retinal pigment epithelial disease. Arch Ophthalmol (Chicago, III 1960). 1984;102(5):675-679.

10. Li D, Kishi S. Restored photoreceptor outer segment damage in multiple evanescent white dot syndrome. Ophthalmology. 2009;116(4):762-770. doi:10.1016/j. ophtha.2008.12.060

11. McCulloch DL, Marmor MF, Brigell MG, et al. ISCEV Standard for full-field clinical electroretinography (2015 update). Doc Ophthalmol. 2015;130(1):1-12. doi:10.1007/ s10633-014-9473-7

12. Brouwer AH, de Wit GC, de Boer JH, van Genderen MM. Effects of DTL electrode position on the amplitude and implicit time of the electroretinogram. Doc Ophthalmol. November 2019. doi:10.1007/s10633-019-09733-3

13. Jabs $D$ a. Standardization of uveitis nomenclature for reporting clinical data. Results of the first international workshop. Am J Ophthalmol. 2005;140(3):509-516. doi:10.1016/j. ajo.2005.03.057

14. Tugal-Tutkun I, Herbort CP, Khairallah M. Scoring of dual fluorescein and ICG inflammatory angiographic signs for the grading of posterior segment inflammation (dual fluorescein and ICG angiographic scoring system for uveitis). Int Ophthalmol. 2010;30(5):539-552. doi:10.1007/s10792-008-9263-x

15. Azarmina M, Moradian S, Azarmina $\mathrm{H}$. The effect of fluorescein angiography on fullfield electroretinography parameters. J Ophthalmic Vis Res. 2012;7(4):300-304. 
Chapter 3

16. Oh KT, Christmas NJ, Folk JC. Birdshot retinochoroiditis: long term follow-up of a chronically progressive disease. Am J Ophthalmol. 2002;133(5):622-629.

17. Tzekov R, Madow B. Visual Electrodiagnostic Testing in Birdshot Chorioretinopathy.J Ophthalmol. 2015;2015:680215. doi:10.1155/2015/680215

18. Berson EL, Sandberg MA, Rosner B, Birch DG, Hanson AH. Natural course of retinitis pigmentosa over a three-year interval. Am J Ophthalmol. 1985;99(3):240-251. doi:10.1016/0002-9394(85)90351-4 
ERG abnormalities in non-infectious uveitis often persist

\section{SUPPLEMENTAL MATERIAL}

Supplemental table 1: ERG results of the 1st $^{\text {and }}$ follow-up ERG (continued on next page)

\begin{tabular}{c|ccc} 
& $\begin{array}{c}\text { 1st ERG } \\
\text { (<1 year of diagnosis) }\end{array}$ & $\begin{array}{c}\text { Follow-up ERG } \\
(<1 \text { year later })\end{array}$ & P-value \\
\hline A-wave amplitude, mean (SD) & & & \\
$\mathrm{cds} / \mathrm{m}^{2} \quad 0.3$ & $-12.52(6.77)$ & $-12.56(6.58)$ & 0,717 \\
1.0 & $-19.80(8.73)$ & $-19.14(7.82)$ & 0,201 \\
3.0 & $-30.35(12.21)$ & $-29.81(11.07)$ & 0,568 \\
10.0 & $-47.26(16.10)$ & $-46.01(15.43)$ & 0,125
\end{tabular}

A-wave implicit time, median [IQR]

$\mathrm{cds} / \mathrm{m}^{2} \quad 0.3$

1.0

3.0

10.0

$19.00[18.50,20.50] \quad 19.00[18.50,20.00] \quad 0,984$

$17.00[16.50,18.00] \quad 17.50[16.50,18.00] \quad 0,502$

$15.50[15.00,16.50] \quad 16.00[15.50,16.50] \quad 0,261$

$15.00[14.50,15.50] \quad 15.00[14.50,15.50] \quad 0,990$

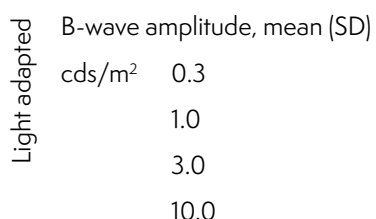

B-wave implicit time, median [IQR]

$\mathrm{cds} / \mathrm{m}^{2} \quad 0.3$

1.0

3.0

10.0

$34.18(15.86)$

$76.00(34.13)$

32.28 (13.58)

0,128

121.46 (48.46)

72.81 (30.38)

0,165

114.65 (41.47)

118.22 (45.11)

0,215

115.13 (39.57)

0,695

$27.00[25.50,30.50] \quad 27.00[26.00,30.38] \quad 0,703$

$29.25[28.00,31.00] \quad 29.25[28.00,31.00] \quad 0,762$

$31.50[30.62,33.50] \quad 32.00[31.00,33.50] \quad 0,410$

$35.50[35.00,37.50] \quad 35.75[35.00,37.00] \quad 0,326$

$30 \mathrm{~Hz}$

Peak amplitude, mean (SD)

Trough implicit time, median $[\mathrm{IQR}]$

$67.31(27.52)$

$68.18(24.94)$

0,463

$22.00[20.50,23.50]$

21.50 [20.50, 23.00]

0,819

Peak implicit time, median [IQR]

$29.50[27.50,31.88]$

$29.00[27.00,31.00]$

0,275

A-wave amplitude, median [IQR]

$\mathrm{cds} / \mathrm{m}^{2} \quad 0.0001$

0.0003

0.001

0.003

$\begin{array}{ll}\frac{0}{0} & 0.01 \\ \frac{0}{0} & 0.03\end{array}$

0.03

0.1

0.3

1.0

3.0

10.0

30.0

$\begin{array}{ccc}-1.73[-6.88,1.18] & -4.24[-12.57,0.71] & 0,454 \\ -1.18[-7.42,4.69] & 0.23[-5.13,3.68] & 0,819 \\ -1.71[-7.60,3.21] & -2.06[-8.64,1.63] & 0,579 \\ -6.39[-13.36,-0.52] & -4.65[-10.33,2.37] & 0,082 \\ -14.36[-26.21,-7.36] & -13.05[-22.74,-3.65] & 0,043 \\ -30.32[-48.85,-17.49] & -28.52[-42.28,-13.20] & 0,047 \\ -46.32[-67.79,-34.05] & -44.00[-59.88,-27.61] & 0,146 \\ -92.76[-136.25,-58.92] & -80.69[-111.45,-56.38] & 0,021 \\ -172.60[-215.60,-110.63] & -152.45[-209.88,-113.97] & 0,179 \\ -92.30[-254.08,-151.47] & -176.85[-233.13,-140.60] & 0,052 \\ -251.55[-307.05,-185.10] & -230.85[-280.22,-190.55] & 0,047 \\ -257.95[-324.75,-192.35] & -238.00[-289.03,-198.20] & 0,053\end{array}$




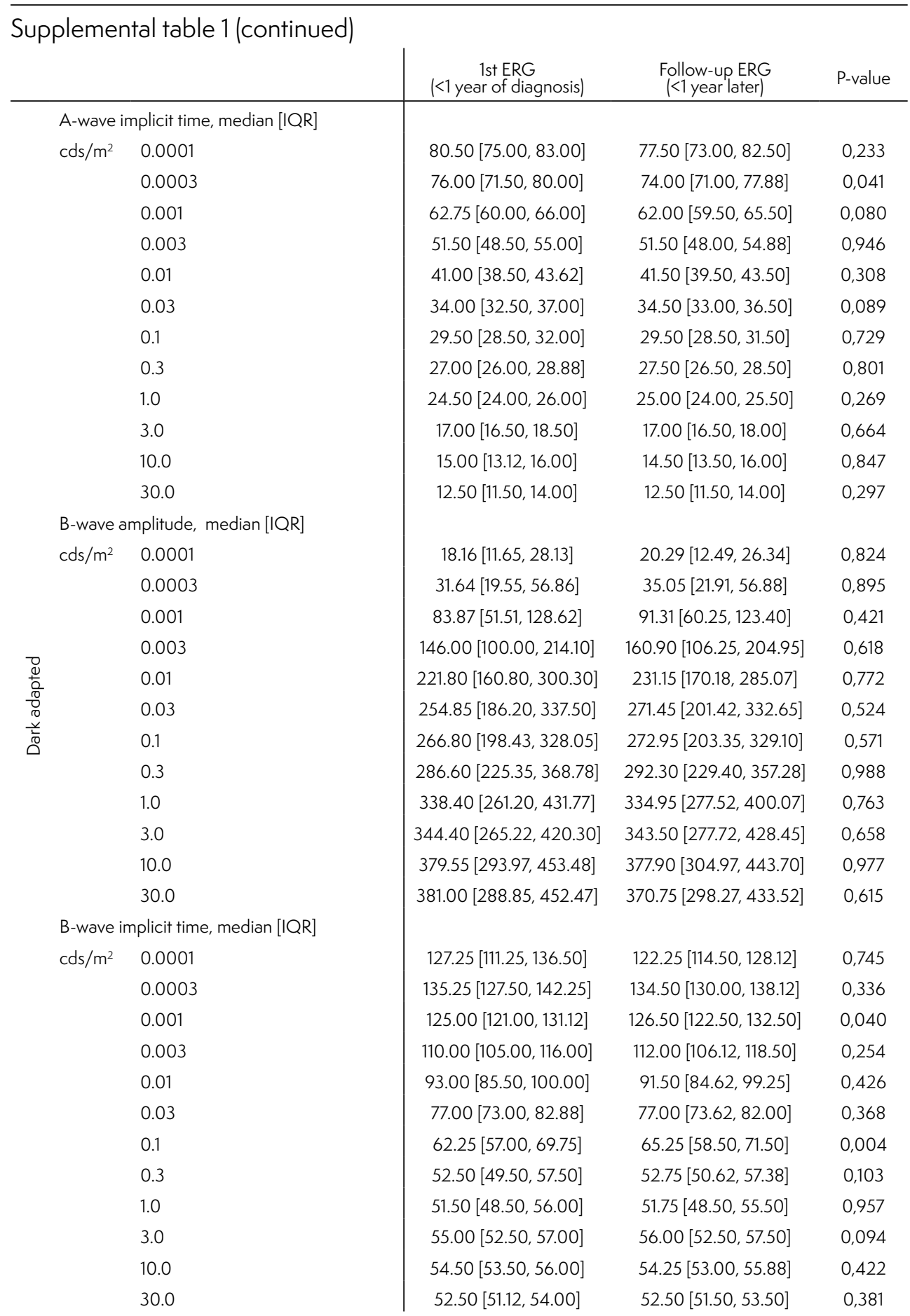

Results of the first and follow-up ERG of the different flash strengths $\left(\mathrm{cds} / \mathrm{m}^{2}\right)$ and adaptation conditions of uveitis patients. Eyes with a pupil size difference $>1 \mathrm{~mm}$ between the first and the follow-up ERG were excluded. $P$-values were tested with the paired t-test or paired Wilcoxon signed rank test depending on the distribution of data. All significances were two-tailed. Abbreviations: $E R G=$ electroretinogram, $S D=$ standard deviation, $I Q R=$ interquartile range, $c d s / \mathrm{m}^{2}=$ candela $\cdot$ seconds $/$ squared meters. 
Supplemental table 2: Fluorescein angiography scores of eyes with a prolonged implicit time of the cone b-wave during their first ERG (continued on next page)

\begin{tabular}{|c|c|c|c|c|c|c|c|c|}
\hline & \multicolumn{4}{|c|}{ At time of first ERG } & \multicolumn{4}{|c|}{ At time of follow-up ERG } \\
\hline & $\begin{array}{c}\text { Improved } \\
\text { at time of } \\
\text { FU ERG } \\
(n=4)\end{array}$ & $\begin{array}{l}\text { Stable at } \\
\text { time of } \\
\text { FU ERG } \\
(n=12)\end{array}$ & $\begin{array}{c}\text { Worsened } \\
\text { at time of } \\
\text { FU ERG } \\
(n=2)\end{array}$ & $p$-value & $\begin{array}{c}\text { Improved } \\
\text { at time of } \\
\text { FU ERG } \\
(n=4)\end{array}$ & $\begin{array}{c}\text { Stable at } \\
\text { time of } \\
\text { FU ERG } \\
(n=3)\end{array}$ & $\begin{array}{c}\text { Worsened } \\
\text { at time of } \\
\text { FU ERG } \\
(n=3)\end{array}$ & $p$-value \\
\hline $\begin{array}{l}\text { FA score, median } \\
{[\mathrm{IQR}]}\end{array}$ & $\begin{array}{l}7.50 \\
{[5.50} \\
8.25]\end{array}$ & $\begin{array}{l}6.00 \\
{[3.75} \\
11.25]\end{array}$ & $\begin{array}{l}3.00 \\
{[3.00} \\
3.00]\end{array}$ & 0.446 & $\begin{array}{l}11.00 \\
{[7.75} \\
12.50]\end{array}$ & $\begin{array}{l}2.00 \\
{[1.50} \\
6.00]\end{array}$ & $\begin{array}{l}20.00 \\
{[10.50} \\
20.00]\end{array}$ & 0.424 \\
\hline Optic disc, N(\%) & & & & 0.597 & & & & 0.050 \\
\hline 0 & $1(25)$ & $3(25)$ & $1(50)$ & & $1(25)$ & $2(67)$ & 0 & \\
\hline 1 & $1(25)$ & $7(58)$ & $1(50)$ & & 0 & $1(33)$ & $1(33)$ & \\
\hline 2 & 0 & $1(8)$ & 0 & & $3(75)$ & 0 & 0 & \\
\hline 3 & $2(50)$ & $1(8)$ & 0 & & 0 & 0 & $2(67)$ & \\
\hline $\begin{array}{l}\text { Cystoid macula } \\
\text { edema, N(\%) }\end{array}$ & & & & 0.254 & & & & 0.257 \\
\hline 0 & 0 & $5(42)$ & $1(50)$ & & $1(25)$ & $1(33)$ & $3(100)$ & \\
\hline 1 & $2(50)$ & $1(8)$ & $1(50)$ & & 0 & $1(33)$ & 0 & \\
\hline 2 & 0 & $1(8)$ & 0 & & $1(25)$ & 0 & 0 & \\
\hline 3 & & & & & 0 & $1(33)$ & 0 & \\
\hline 4 & $2(50)$ & $5(42)$ & $0(0)$ & & $2(50)$ & 0 & 0 & \\
\hline $\begin{array}{l}\text { Vasculitis posterior } \\
\text { pole, } \mathrm{N}(\%)\end{array}$ & & & & 0.766 & & & & 0.571 \\
\hline 0 & $3(75)$ & $6(50)$ & $1(50)$ & & $3(75)$ & $2(67)$ & $1(33)$ & \\
\hline 1 & 0 & $1(8)$ & $1(50)$ & & $1(25)$ & 0 & 0 & \\
\hline 2 & $1(25)$ & $3(25)$ & 0 & & 0 & $1(33)$ & $2(67)$ & \\
\hline 3 & 0 & $2(17)$ & 0 & & 0 & 0 & 0 & \\
\hline $\begin{array}{l}\text { Vasculitis } \\
\text { quadrants, } N(\%)\end{array}$ & & & & $>.999$ & & & & 0.829 \\
\hline 0 & $3(75)$ & $5(42)$ & $2(100)$ & & $1(25)$ & 0 & $1(33)$ & \\
\hline 1 & 0 & $1(8)$ & 0 & & $1(25)$ & $2(66.7)$ & 0 & \\
\hline 2 & 0 & $2(17)$ & 0 & & $1(25)$ & 0 & 0 & \\
\hline 3 & 0 & $1(8)$ & 0 & & 0 & 0 & 0 & \\
\hline 4 & $1(25)$ & $3(25)$ & 0 & & $1(25)$ & $1(33)$ & $2(67)$ & \\
\hline $\begin{array}{l}\text { Capp. leakage } \\
\text { posterior pole, } \\
\mathrm{N}(\%)\end{array}$ & & & & 0.652 & & & & 0.371 \\
\hline 0 & $3(75)$ & $8(67)$ & $1(50)$ & & $1(25)$ & $3(100)$ & $1(33)$ & \\
\hline 1 & 0 & $3(25)$ & $1(50)$ & & $1(25)$ & 0 & 0 & \\
\hline 2 & $1(25)$ & $1(8)$ & 0 & & $2(50)$ & $0(0)$ & $2(67)$ & \\
\hline $\begin{array}{l}\text { Focal capp. } \\
\text { leakage quadrants, } \\
\mathrm{N}(\%)\end{array}$ & & & & $>.999$ & & & & 0.167 \\
\hline 0 & $4(100)$ & $10(83)$ & $2(100)$ & & $1(25)$ & $3(100)$ & $3(100)$ & \\
\hline 1 & 0 & 0 & 0 & & $1(25)$ & 0 & 0 & \\
\hline 2 & 0 & $1(8)$ & 0 & & $2(50)$ & 0 & 0 & \\
\hline 3 & 0 & $1(8)$ & 0 & & 0 & 0 & 0 & \\
\hline
\end{tabular}


Supplemental table 2 (continued)

\begin{tabular}{|c|c|c|c|c|c|c|c|c|}
\hline & \multicolumn{4}{|c|}{ At time of first ERG } & \multicolumn{4}{|c|}{ At time of follow-up ERG } \\
\hline & $\begin{array}{c}\text { Improved } \\
\text { at time of } \\
\text { FU ERG } \\
(n=4)\end{array}$ & $\begin{array}{l}\text { Stable at } \\
\text { time of } \\
\text { FU ERG } \\
(n=12)\end{array}$ & $\begin{array}{c}\text { Worsened } \\
\text { at time of } \\
\text { FU ERG } \\
(n=2)\end{array}$ & $p$-value & $\begin{array}{c}\text { Improved } \\
\text { at time of } \\
\text { FU ERG } \\
(n=4)\end{array}$ & $\begin{array}{l}\text { Stable at } \\
\text { time of } \\
\text { FU ERG } \\
(n=3)\end{array}$ & $\begin{array}{c}\text { Worsened } \\
\text { at time of } \\
\text { FU ERG } \\
(n=3)\end{array}$ & $p$-value \\
\hline $\begin{array}{l}\text { Diffuse capp. } \\
\text { leakage quadrants, } \\
\mathrm{N}(\%)\end{array}$ & & & & $>.999$ & & & & 0.133 \\
\hline 0 & $4(100)$ & $11(92)$ & $2(100)$ & & $4(100)$ & $3(100)$ & $1(33)$ & \\
\hline 4 & 0 & 0 & 0 & & 0 & 0 & 0 & \\
\hline 8 & 0 & $1(8)$ & 0 & & 0 & 0 & $2(67)$ & \\
\hline $\begin{array}{l}\text { non perfusion } \\
\text { macula, } N(\%)\end{array}$ & & & & NA & & & & $>.999$ \\
\hline 0 & $4(100)$ & $12(100)$ & $2(100)$ & & $3(75)$ & $3(100)$ & $3(100)$ & \\
\hline 1 & 0 & 0 & 0 & & $1(25)$ & 0 & 0 & \\
\hline $\begin{array}{l}\text { non perfusion } \\
\text { posterior pole, } \\
\mathrm{N}(\%)\end{array}$ & & & & NA & & & & 0.133 \\
\hline 0 & $4(100)$ & $12(100)$ & $2(100)$ & & $4(100)$ & $3(100)$ & $1(33)$ & \\
\hline 1 & 0 & 0 & 0 & & 0 & 0 & $2(67)$ & \\
\hline $\begin{array}{l}\text { non perfusion } \\
\text { quadrants, } \mathrm{N}(\%)\end{array}$ & & & & & & & & $>.999$ \\
\hline 0 & $4(100)$ & $11(92)$ & $2(100)$ & $>.999$ & $3(75)$ & $3(100)$ & $1(33)$ & \\
\hline 2 & 0 & $1(8)$ & 0 & & $1(25)$ & 0 & $0(0)$ & \\
\hline $\begin{array}{l}\text { neovascularisation } \\
\text { elsewhere, } \mathrm{N}(\%)\end{array}$ & & & & $>.999$ & & & & NA \\
\hline 0 & $4(100)$ & $11(92)$ & $2(100)$ & & $4(100)$ & $12(100)$ & $2(100)$ & \\
\hline 2 & 0 & $1(8)$ & 0 & & 0 & 0 & 0 & \\
\hline $\begin{array}{l}\text { inpoint leakage, } \\
\mathrm{N}(\%)\end{array}$ & & & & 0.111 & & & & NA \\
\hline 0 & $4(100)$ & $12(100)$ & $1(50)$ & & $4(100)$ & $12(100)$ & $2(100)$ & \\
\hline 2 & 0 & 0 & $1(50)$ & & 0 & 0 & 0 & \\
\hline
\end{tabular}

FAs of uveitis patients with a prolonged implicit time of the cone b-wave during their first ERG were scored using the Fluorescein angiographic scoring system of the Angiography Scoring for Uveitis Working Group (ASUWOG). Differences in FA score were compared between eyes with a stable, improved, or worsened implicit time of the cone b-wave. FAs were performed up to 3 months around the ERG. The first ERG was performed in the first year of inflammation, the follow-up ERG one year later. FAs with the highest overall FA score until the ERG was recorded are shown. Parts of the FA score were no leakage was visible (neovascularization optic disc and pooling) are not shown. Eyes with a pupil size difference $>1 \mathrm{~mm}$ between the first and the follow-up ERG were excluded. Abbreviations: $F A=$ Fluorescein angiogram, $N=$ number, $I Q R=$ interquartile range, capp. = capillary.

Supplemental table 3 (right): FAs of uveitis patients with a normal implicit time of the cone b-wave during their first ERG were scored using the Fluorescein angiographic scoring system of the Angiography Scoring for Uveitis Working Group (ASUWOG). Differences in FA score were compared between eyes with a stable or worsened implicit time of the cone $b$-wave. FAs were performed up to 3 months around the ERG. The first $E R G$ was performed in the first year of inflammation, the follow-up ERG one year later. FAs with the highest overall FA score until the ERG was recorded are shown. Parts of the FA score were no leakage was visible (neovascularization optic disc, neovascularization elsewhere, pinpoint leakage, non-perfusion macula, nonperfusion posterior pole, and pooling) are not shown. Eyes with a pupil size difference $>1 \mathrm{~mm}$ between the first and the follow-up ERG were excluded. Abbreviations: $E R G=$ electroretinogram, FA = Fluorescein angiogram, $N=$ number, $I Q R=$ interquartile range, capp. = capillary. 
Supplemental table 3: Fluorescein angiographic scores of eyes with a normal implicit time of the cone b-wave during their first ERG

\begin{tabular}{|c|c|c|c|c|c|c|}
\hline & \multicolumn{3}{|c|}{ At time of first ERG } & \multicolumn{3}{|c|}{ At time of follow-up ERG } \\
\hline & $\begin{array}{l}\text { Stable at time } \\
\text { of } \\
\text { FU ERG (n } \\
=18 \text { ) }\end{array}$ & $\begin{array}{l}\text { Worsened at } \\
\text { time of } F U \\
\text { ERG }(n=4)\end{array}$ & $p$-value & $\begin{array}{l}\text { Stable at time } \\
\text { of } \\
\text { FU ERG ( } n=11)\end{array}$ & $\begin{array}{l}\text { Worsened at } \\
\text { time of FU } \\
\text { ERG }(n=1)\end{array}$ & $p$-value \\
\hline FA score, median [IQR] & $4.00[0.00,6.75]$ & $1.00[0.00,2.50]$ & 0.205 & $0.00[0.00,1.50]$ & $0.00[0.00,0.00]$ & 0.568 \\
\hline Optic disc, N(\%) & & & 0.852 & & & $>.999$ \\
\hline 0 & $7(39)$ & $3(75)$ & & $9(82)$ & $1(100)$ & \\
\hline 1 & $4(22)$ & $0(0)$ & & $1(9)$ & 0 & \\
\hline 2 & $5(28)$ & $1(25)$ & & $1(9)$ & 0 & \\
\hline 3 & $2(11)$ & $0(0)$ & & & & \\
\hline $\begin{array}{l}\text { Cystoid macula edema, } \\
\mathrm{N}(\%)\end{array}$ & & & $>.999$ & & & $>.999$ \\
\hline 0 & $13(72)$ & $4(100)$ & & 10 & $1(100)$ & \\
\hline 1 & $3(17)$ & $0(0)$ & & 0 & 0 & \\
\hline 2 & 0 & 0 & & $1(9)$ & 0 & \\
\hline 3 & $1(6)$ & $0(0)$ & & 0 & 0 & \\
\hline 4 & $1(6)$ & $0(0)$ & & 0 & 0 & \\
\hline $\begin{array}{l}\text { Vasculitis posterior } \\
\text { pole, } \mathrm{N}(\%)\end{array}$ & & & $>.999$ & & & $>.999$ \\
\hline 0 & $11(61)$ & $4(100)$ & & 10 & $1(100)$ & \\
\hline 1 & $2(11)$ & $0(0)$ & & & 0 & \\
\hline 2 & $3(17)$ & $0(0)$ & & $1(9)$ & 0 & \\
\hline 3 & $2(11)$ & $0(0)$ & & & 0 & \\
\hline $\begin{array}{l}\text { Vasculitis quadrants, } \\
\mathrm{N}(\%)\end{array}$ & & & 0.419 & & & $>.999$ \\
\hline 0 & $11(61)$ & $2(50)$ & & $8(73)$ & $1(100)$ & \\
\hline 1 & $1(6)$ & $0(0)$ & & $1(9)$ & 0 & \\
\hline 2 & $2(11)$ & $2(50)$ & & $1(9)$ & 0 & \\
\hline 3 & $2(11)$ & $0(0)$ & & 0 & 0 & \\
\hline 4 & $2(11)$ & $0(0)$ & & $1(9)$ & 0 & \\
\hline $\begin{array}{l}\text { Capp. leakage posterior } \\
\text { pole, } \mathrm{N}(\%)\end{array}$ & & & $>.999$ & & & $>.999$ \\
\hline 0 & 17 & $4(100)$ & & & $1(100)$ & \\
\hline 1 & $1(6)$ & $0(0)$ & & $1(9)$ & $0(0)$ & \\
\hline $\begin{array}{l}\text { Focal capp. leakage } \\
\text { quadrants, } N(\%)\end{array}$ & & & $>.999$ & & & NA \\
\hline 0 & & $4(100)$ & & $11(100)$ & $1(100)$ & \\
\hline 4 & $1(6)$ & $0(0)$ & & 0 & 0 & \\
\hline $\begin{array}{l}\text { Diffuse capp. leakage } \\
\text { quadrants, } \mathrm{N}(\%)\end{array}$ & & & $>.999$ & & & NA \\
\hline 0 & & $4(100)$ & & $11(100)$ & $1(100)$ & \\
\hline 4 & $2(11)$ & $0(0)$ & & 0 & 0 & \\
\hline $\begin{array}{l}\text { non perfusion } \\
\text { quadrants, } \mathrm{N}(\%)\end{array}$ & & & NA & & & \\
\hline 0 & $18(100)$ & $4(100)$ & & 10 & $1(100)$ & $>.999$ \\
\hline 2 & & & & $1(9)$ & $0(0)$ & \\
\hline FAGNVOpD = $0(\%)$ & $18(100)$ & $4(100)$ & NA & & & \\
\hline FAGNVE = $0(\%)$ & $18(100)$ & $4(100)$ & NA & & & \\
\hline FAGpinL = $0(\%)$ & $18(100)$ & $4(100)$ & NA & & & \\
\hline FAGpool = $0(\%)$ & $18(100)$ & $4(100)$ & NA & & & \\
\hline FAGcapNPMac $=0(\%)$ & $18(100)$ & $4(100)$ & NA & $11(100)$ & $1(100)$ & NA \\
\hline FAGcapNPPP = $0(\%)$ & $18(100)$ & $4(100)$ & NA & $11(100)$ & $1(100)$ & NA \\
\hline
\end{tabular}


Supplemental table 4: Correlation matrix of significant clinical factors, as well as systemic medication, duration of uveitis, and age

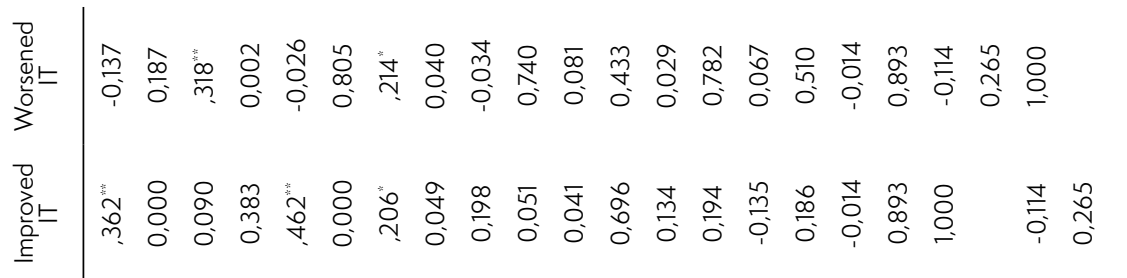

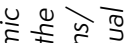

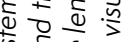

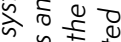

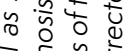

बे

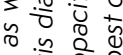

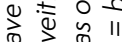

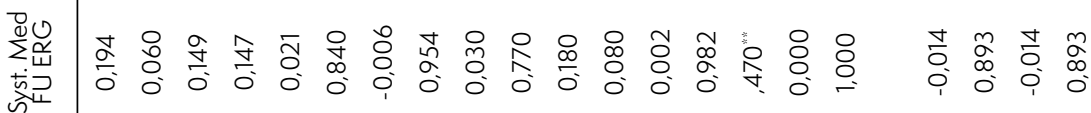

1.

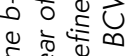

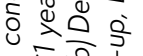

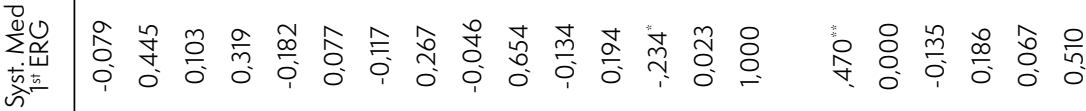

8 v

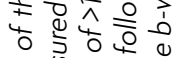

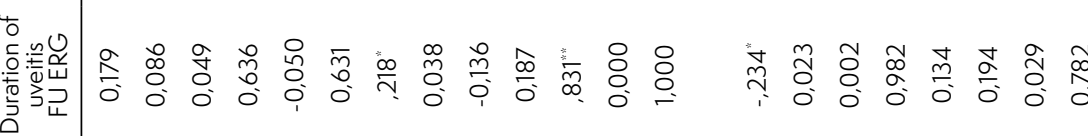

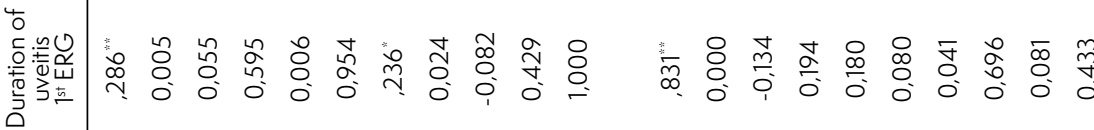

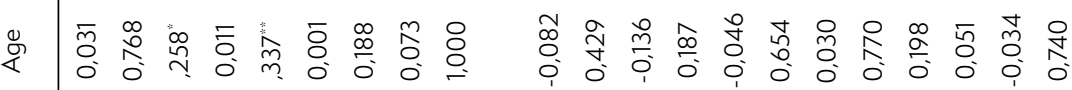

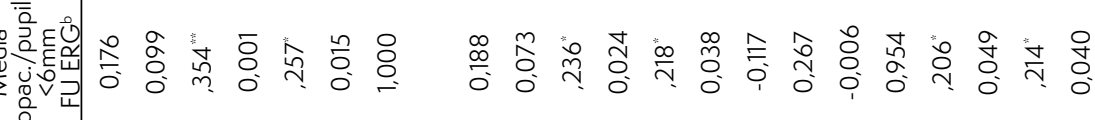

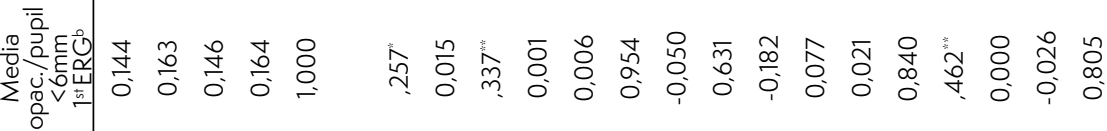

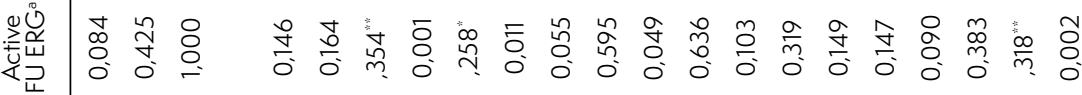

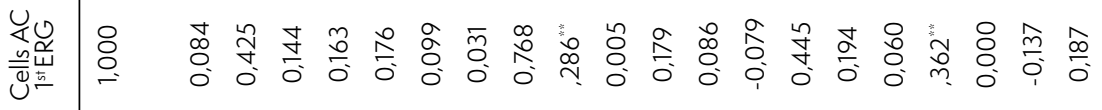

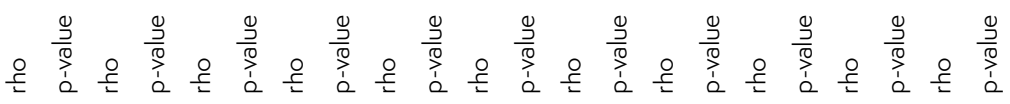

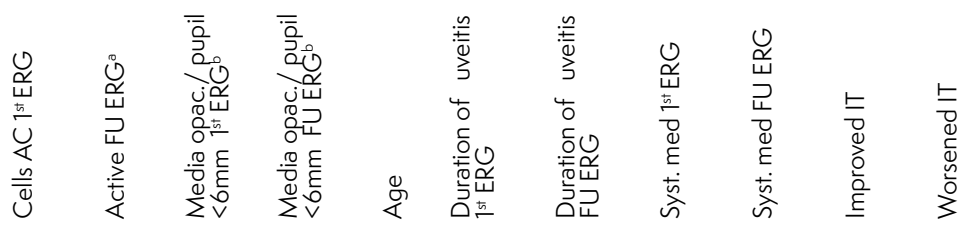

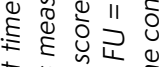

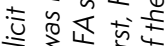

हो)

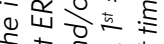

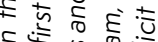

पष $\frac{\omega}{\sigma} \overline{0}$

of 0 에

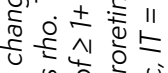

들

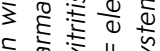

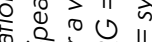

응 운

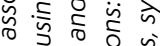

t 0 눙

表

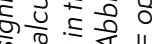

जि 0

خ छ

Ч

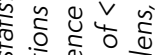

万人

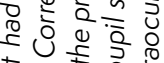

to 0

"5 0 \%

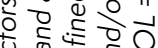

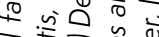

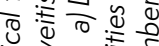

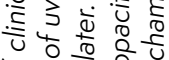

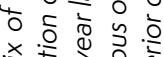

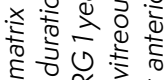

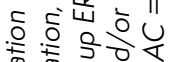

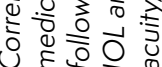


ERG abnormalities in non-infectious uveitis often persist 


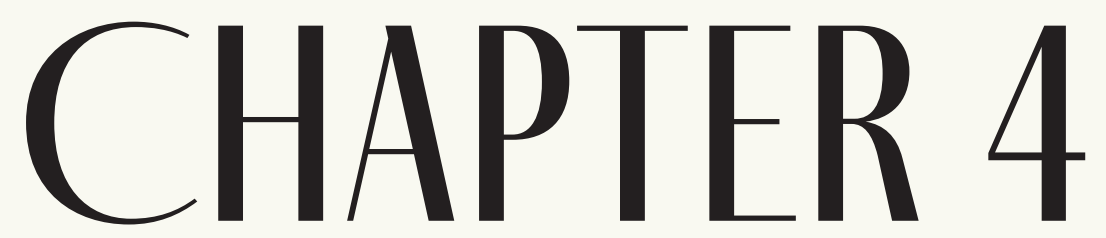

\section{PROLONGED CONE B-WAVE IMPLICIT TIME ON ELECTRORETINOGRAPHY IN RELATION TO RETINAL LAYER ANALYSIS}

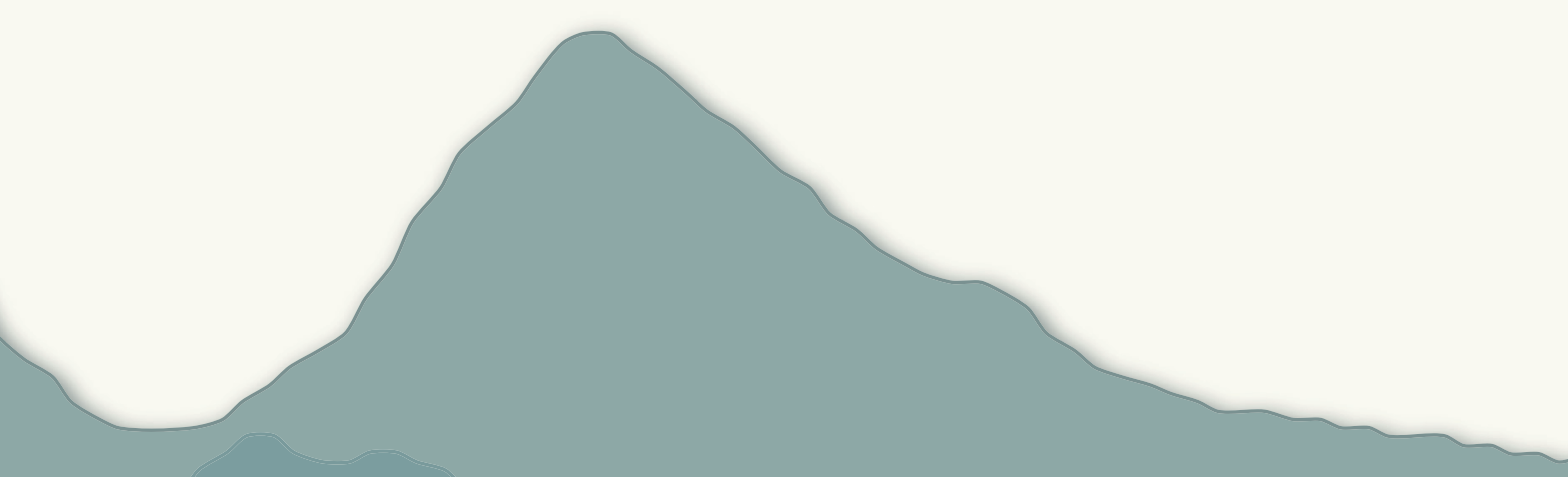

Anna H Brouwer Vincent JM Hortensius Gerard C de Wit Ninette $\mathrm{H}$ ten Dam

Ralph Wijnhoven Maria M van Genderen Joke $\mathrm{H}$ de Boer 


\section{ABSTRACT}

\section{Purpose}

To identify possible correlations between structural changes on optical coherence tomography (OCT) and functional abnormalities on electroretinography (ERG) in uveitis patients. A characteristic ERG abnormality in uveitis is a prolonged cone b-wave implicit time (cone b-IT). We investigated the relationship between a prolonged cone b-IT and retinal layer thickness on OCT. Differences in thickness were expected to occur in the retinal layers which contain the bipolar cells: the inner plexiform layer (IPL), inner nuclear layer (INL), and outer plexiform layer (OPL).

\section{Methods}

OCT scans of 189 uveitis patients (333 eyes) were segmented with lowa reference algorithms software. Differences in mean thickness were analyzed of ten retinal layers per region of the ETDRS grid between non-uveitis eyes and uveitis eyes with and without a prolonged cone b-IT on ERG. Spearman's rho correlation coefficients were also analyzed.

\section{Results}

The IPL was significantly thinner $(p<0.05$,regions $2,3,5-9)$ in eyes with a prolonged cone b-IT, whereas the INL was significantly thicker $(p<0.05$, regions 1 - 6). No differences were observed in the OPL. However, the correlation coefficients between implicit times and retinal layer thickness were weak (Spearman's rho $0.103-0.388$, $p<0.05)$. Vitritis, FA score, and age were also weakly correlated to thickness.

\section{Conclusions}

There is a weak correlation between a prolonged cone b-IT on ERG and a thickening of the INL and thinning of the IPL. Other clinical factors including age and uveitis activity showed similar weak correlations to thickness. Therefore, differences in retinal thickness alone do not fully explain a prolonged cone b-IT. 


\section{INTRODUCTION}

Uveitis is a relatively rare disease with a significant visual impact. The prevalence is estimated at 121 cases per 100.000 adults in the United States. However, uveitis causes a relatively large percentage of $10 \%$ of blindness in the working population., Multiple treatment strategies are available with immunomodulating treatments, but still several complications can develop, including glaucoma, cystoid macula edema (CME), and retinal atrophy. ${ }^{3,4}$

Recently we described a characteristic abnormality on the electroretinogram (ERG) in uveitis patients: a prolonged cone b-wave implicit time (cone b-IT). ${ }^{5}$ This ERG abnormality was present in all anatomical subtypes of uveitis and correlated to the severity of the inflammation. In most cases a prolonged cone b-IT persisted even when the inflammation had become quiescent, indicating a possible permanent loss of retinal function. ${ }^{6}$

We investigated a possible association between retinal function and retinal structure using automatic segmentation of the retinal layers on optical coherence tomography (OCT) images. Automatic segmentation is constantly improving, and associations between the thickness of retinal layers and clinical functional parameters have been described. For instance, in glaucoma and diabetic retinopathy a correlation was found between thinning of layer thickness and visual fields, and retinopathy stage, respectively. ${ }^{7-10}$

We investigated possible differences in retinal layer thickness between nonuveitis eyes and uveitis eyes with and without a prolonged cone b-IT. Because the cone b-wave is mainly generated by the ON and OFF bipolar cells, ${ }^{11}$ we expected changes in thickness to occur in the retinal layers which correspond to the photoreceptor-bipolar cell synaps and the bipolar cells: the outer plexiform layer (OPL), inner nuclear layer (INL) and the inner plexiform layer (IPL). However, associations between the thickness of other retinal layers and the cone b-IT were investigated as well.

\section{METHODS}

\section{Patients}

We included 189 patients from a previously described cohort of 200 uveitis patients, who had an OCT on the same day as an ERG measurement and a clinical visit. ${ }^{5,6}$ All patients were mentally competent, $\geq 18$ years of age, and gave informed consent to participate. Patients were all treated at the University Medical Center Utrecht, a tertiary referral center for uveitis. Patients with uveitis associated with juvenile idiopathic 
arthritis or other retinal diseases including diabetic retinopathy, retinal dystrophy, family history of retinal dystrophy, myopic degeneration, or severe media opacities were excluded.

Ethical approval was requested and obtained from the Medical Ethical Research Committee of the University Medical Center Utrecht. This study was conducted in compliance with the ethical principles of the declaration of Helsinki.

\section{ERG measurement}

ERGs were measured according to an extended protocol, with more flash strengths than the standard International Society for Clinical Electrophysiology of Vision (ISCEV) protocol.11 For the light-adapted (LA) ERG this protocol comprised 0.3, 1.0, 3.0 and $10.0 \mathrm{cds} / \mathrm{m}^{2}$ flash strengths and a $30 \mathrm{~Hz}$ flicker response. Dawson-Trick-Litzkow (DTL) electrodes were used as active electrodes, and an Espion E3 system with Colordome stimulator (Diagnosys LLC, Cambridge, UK) for flash stimulation. We defined the cone b-IT as prolonged when two or more consecutive flash strengths from the LA ERG were above the $95^{\text {th }}$ percentile of the implicit times of a normal population. ${ }^{5,11}$ Our reference values, and ERG results have been previously described.5,6

\section{OCT retinal layer analysis}

OCT images were made with Carl Zeiss Cirrus HD-OCT model 5000 (Carl Zeiss Meditec AG, Germany). C-scans were $200 \times 200 \times 1024$ voxels and were analyzed using the lowa Reference Algorithms (Retinal Image Analysis Lab, lowa Institute for Biomedical Imaging, lowa City, IA).712,13 This program automatically defines the borders of ten retinal layers and calculates the mean (SD) thickness of these retinal layers per retinal region.

Figure 1 gives an overview of the ten retinal layers as seen on $O C T$ : the retinal nerve fiber layer (RNFL), ganglion cell layer (GCL), inner plexiform layer (IPL), inner nuclear layer (INL), outer plexiform layer (OPL), outer nuclear layer (ONL), inner segment/ outer segment (IS/OS), outer segment layer (OSL), outer segment photoreceptors/ retinal pigment epithelium complex (OPR) and retinal pigment epithelium (RPE). The nine regions of which the thickness were analyzed were those of the Early Treatment Diabetic Retinopathy Study (ETRDRS) grid.

\section{Statistical analysis}

R Studio (R studio, 2019, version 3.6.0) was used for statistical analyses. The distribution of the automatically segmented thickness analysis was evaluated with Shapiro-Wilk tests, Q-Q plots, histograms and boxplots. Extreme outliers (Tukey's $k=3$ ) were 


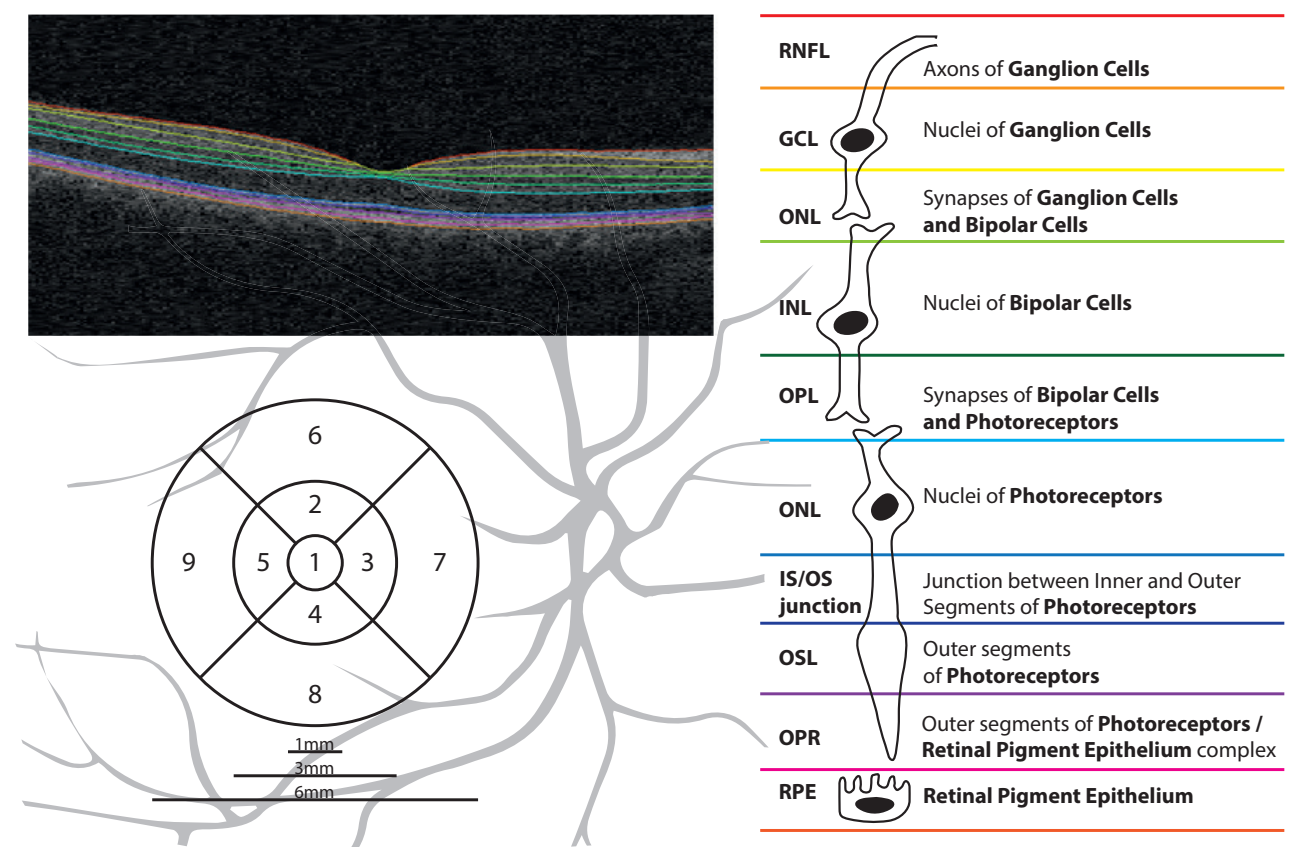

Figure 1: Example of the ten segmented retinal layers and the Early Treatment Diabetic Retinopathy Study (ETDRS) grid. On the right a schematic representation of the different cell types per layer. Abbreviations: RNFL = retinal nerve fiber layer, $G C L=$ ganglion cell layer, $I P L=$ inner plexiform layer, INL = inner nuclear layer, $O P L$ = outer plexiform layer, ONL = outer nuclear layer, IS/OS junction = inner segment/outer segment junction, OSL = outer segment layer, $O P R=$ outer segment photoreceptors/retinal pigment epithelium complex (OPR) and retinal pigment epithelium (RPE).

identified, and the segmentation of these OCT images was manually checked and corrected when necessary.

After manual correction, the thickness analysis was performed again and the thicknesses of the different retinal layers were compared between uveitis eyes with a prolonged cone b-IT, uveitis eyes with a normal cone b-IT, and eyes with no uveitis (of patients with a unilateral uveitis). Also, correlations between the implicit times of the cone b-wave of the different flash strengths $\left(0.3,1.0,3.0\right.$ and $\left.10.0 \mathrm{cds} / \mathrm{m}^{2}\right)$ in $\mathrm{ms}$ and thicknesses in microns were investigated. We considered $p$-values of $<0.05$ as statistically significant. All tests were 2-łailed.

Next, layer thickness were investigated in relation to clinical parameters that were associated with a prolonged cone b-IT including: fluorescein angiography (FA) scores (graded according to the Angiography Scoring for Uveitis Working Group (ASUWOG), ${ }^{14}$ the presence of cells in the anterior chamber (trace or more), or vitreous (1+ or more), as graded according the standardization of uveitis nomenclature (SUN) criteria, ${ }^{15}$ and age, using Spearman's rho coefficient. 


\section{$\underline{\text { RESULTS }}$}

\section{Patient population}

A total of 372 eyes of 189 patients were included. Two of the initial 200 uveitis patients of our cohort were excluded because the OCT scan was not performed on the same day as the ERG. Seven patients were excluded because their OCT scan was performed with a different OCT device (Heidelberg). Lastly, two patients were excluded because the algorithm could not properly segment the retinal layers in their eyes, due to a severe macula pucker or CME.

In five patients with a bilateral uveitis, one eye was excluded because the algorithm could not properly segment the retinal layers of one eye. There were 40 uveitis patients with a unilateral uveitis. In one of these, there was only an OCT scan available of the uveitis eye. This enabled us to investigate 39 non-uveitis eyes of patients with a unilateral uveitis.

Table 1 gives an overview of the patient characteristics and diagnoses. Of the 333 uveitis eyes, 142 eyes $(42.6 \%)$ had a prolonged cone b-IT. None of the nonuveitis eyes ( $n=39$ ) of patients with a unilateral uveitis had a prolonged cone b-IT. Patients with a prolonged cone b-IT were on average older (median 61.2 (IQR 51.2 - 70.1) years) compared to patients with a normal cone b-IT (median 44.8 (IQR 33.7 - 55.7) years) and patients with unilateral uveitis (median 47.1 (IQR 32.4 -56.3) years)) ( $p<0.001)$. There was no differences based on gender ( $p=0.320)$. Most patients had a bilateral uveitis $(\mathrm{n}=$ $149(78.8 \%)$ ). A prolonged cone b-IT was equally observed in unilateral and bilateral uveitis. There were no differences between eyes with and without a prolonged cone b-IT and uveitis diagnosis ( $p=0.198$ ), or anatomical localization of uveitis $(p=$ 0.802).

Abbreviations: $N=$ number, $I Q R=$ interquartile range, $M S=$ multiple sclerosis, $H L A$ B27 = human leucocyte antigen B27, VKH = VogtKoyanagi-Harada.

Table 1: Patient characteristics

\begin{tabular}{l|c} 
& Patients $(\mathrm{n}=189)$ \\
\hline Male gender, N (\%) & $57(30.2)$ \\
Age, median [IQR] & $53.65[39.16,64.07]$ \\
Bilateral uveitis, N (\%) & $149(78.8)$ \\
Diagnosis, N (\%) & \\
Unknown cause & $76(40.2)$ \\
Sarcoidosis & $29(15.3)$ \\
Chorioretinitis & $27(14.3)$ \\
Birdshot & $26(13.8)$ \\
HLA-B27 & $14(7.4)$ \\
MS & $7(3.7)$ \\
Behçet & $5(2.6)$ \\
VKH & $5(2.6)$
\end{tabular}




\section{OCT thickness analysis}

Table 2 shows in shaded grey the results of the IPL, INL, and OPL, the layers which encompass the bipolar cells. In eyes with a prolonged cone b-IT, the mean thickness of the IPL was significantly thinner in seven regions of the ETDRS grid (regions 2, 3, 5 - 9). In contrast, the INL layer was significantly thicker in the six central regions of the ETDRS grid (regions 1 - 6) in eyes with a prolonged cone b-IT. No differences were observed in the OPL.

Table 3 gives an overview of Spearman's rho correlation coefficients of implicit times in ms of the different flash strengths in relation to the thickness in microns of the IPL and INL. In line with the findings in Table 2, these correlations between thickness and implicit time were often statistically significant, but the correlation coefficients were weak ranging between 0.103 and 0.388 . Weak correlations were also found between the thickness of the INL and IPL and clinical parameters that were previously found to be associated with a prolonged cone b-IT (Table 4). ${ }^{5}$

Besides the retinal layers which contain the bipolar cells, other layers also showed significant differences in thickness in eyes with a prolonged cone b-IT (Table 2). The retinal nerve fiber layer (RNFL) was significantly thicker (Table 2). Glaucoma, a common complication of uveitis, was investigated as a possible confounder for the differences in the RNFL thickness. Eyes with glaucoma ( $n=67$ eyes) had a significantly thicker RNFL in regions 1, 7 and 9 and a thinner GCL and IPL in regions 2 to 9.

Eyes with glaucoma also had a thicker INL (region 3, 7), similarly to eyes with a prolonged cone b-IT. However, when eyes with glaucoma were excluded and a comparison was made between eyes with a prolonged cone b-IT, a normal cone b-IT, and non-uveitis eyes, the IPL was still significantly thinner and the INL was thicker in eyes with a prolonged cone b-IT. The other layers also showed similar outcomes when glaucoma eyes were excluded.

The outer retinal layers showed significant differences as well (Table 2). The largest differences were observed between the non-uveitis eyes and uveitis eyes with a prolonged cone b-IT. The results of the uveitis eyes with a normal cone b-IT lay in between, but their retinal layer thicknesses were closer to those of the non-uveitis eyes. When the non-uveitis eyes were excluded, and only the differences between uveitis eyes with and without a prolonged cone b-IT were compared, similar outcomes were observed. Regarding the layers containing bipolar cells, the only different outcome was that the INL was no longer significantly thicker in region 3. 
Table 2: Thickness analysis of the retinal layers of the different regions of the ETDRS grid of uveitis eyes with and without a prolonged cone b-wave, and non-uveitis eyes.

\begin{tabular}{|c|c|c|c|c|c|c|}
\hline & & Mean thickness & region 1 & region 2 & region 3 & region 4 \\
\hline \multirow{8}{*}{ 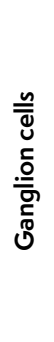 } & \multirow{4}{*}{ RNFL } & no uveitis & $5.20[3.42,8.28]$ & $22.98[21.38,26.17]$ & $27.29[24.49,30.19]$ & $25.38[21.91,28.73]$ \\
\hline & & uveitis normal b-IT & $6.69[3.86,13.09]$ & $24.74[21.55,30.91]$ & $29.09[26.03,33.30]$ & $24.84[21.11,29.89]$ \\
\hline & & prolonged b-IT & $11.48[7.50,20.13]$ & $28.45[23.67,36.21]$ & $34.58[28.65,41.81]$ & $28.59[23.13,35.55]$ \\
\hline & & $p$-value & $<0.001$ & $<0.001$ & $<0.001$ & $<0.001$ \\
\hline & \multirow{4}{*}{$\mathrm{GCL}$} & no uveitis & $17.16[13.60,22.48]$ & $52.35[45.59,56.27]$ & $54.31[48.99,58.54]$ & $52.75[47.95,56.11]$ \\
\hline & & uveitis normal b-IT & $18.29[13.48,23.40]$ & $50.48[45.12,55.94]$ & $52.50[47.16,58.31]$ & $51.20[44.81,57.44]$ \\
\hline & & prolonged b-IT & $18.80[12.40,29.87]$ & $49.66[38.89,57.19]$ & $51.75[39.96,57.03]$ & $49.78[40.78,58.82]$ \\
\hline & & $p$-value & 0.517 & 0.233 & 0.098 & 0.511 \\
\hline \multirow{12}{*}{$\begin{array}{l}\underline{n} \\
\overline{0} \\
\frac{0}{0} \\
\frac{0}{0} \\
\frac{0}{0}\end{array}$} & \multirow{4}{*}{ IPL } & no uveitis & $26.92[24.08,28.94]$ & $38.53[35.66,40.46]$ & $41.64[36.03,44.48]$ & $37.24[35.11,40.65]$ \\
\hline & & uveitis normal b-IT & $26.59[22.47,29.50]$ & $36.39[33.23,40.78]$ & $39.25[35.13,43.77]$ & $36.67[32.34,40.42]$ \\
\hline & & prolonged b-IT & $27.11[23.17,30.27]$ & $35.02[30.47,40.00]$ & $36.70[31.84,41.56]$ & $35.86[30.49,39.75]$ \\
\hline & & $p$-value & 0.443 & 0.004 & 0.006 & 0.175 \\
\hline & \multirow{4}{*}{ INL } & no uveitis & $20.83[17.05,23.17]$ & $36.47[33.91,39.89]$ & $40.60[37.70,43.02]$ & $37.62[35.45,41.20]$ \\
\hline & & \multirow{2}{*}{$\begin{array}{l}\text { uveitis normal b-IT } \\
\text { prolonged b-IT }\end{array}$} & $21.73[17.67,27.61]$ & $38.04[34.41,41.94]$ & $42.61[39.02,45.82]$ & $38.75[34.84,43.00]$ \\
\hline & & & $26.20[21.17,37.83]$ & $40.75[36.11,47.26]$ & $43.42[38.41,48.54]$ & $41.35[36.74,48.85]$ \\
\hline & & \multirow{2}{*}{$\begin{array}{l}\text { p-value } \\
\text { no uveitis }\end{array}$} & $<0.001$ & $<0.001$ & 0.043 & $<0.001$ \\
\hline & \multirow{4}{*}{ OPL } & & $23.92[20.34,26.66]$ & $27.05[24.71,30.66]$ & $29.04[26.52,34.81]$ & $30.44[26.00,35.96]$ \\
\hline & & \multirow{3}{*}{$\begin{array}{ll}\text { uveitis normal b-IT } \\
\text { prolonged b-IT }\end{array}$} & $22.20[17.97,26.68]$ & $27.96[24.91,32.59]$ & $29.20[25.57,33.49]$ & $27.93[25.04,34.69]$ \\
\hline & & & $22.93[17.18,28.62]$ & $28.49[24.82,34.42]$ & $29.25[24.24,34.73]$ & $27.78[24.97,34.32]$ \\
\hline & & & 0.466 & 0.647 & 0.509 & 0.286 \\
\hline \multirow{15}{*}{$\begin{array}{l}\frac{n}{2} \\
\frac{0}{0} \\
\frac{0}{0} \\
\frac{0}{0} \\
\frac{0}{0} \\
\frac{0}{\alpha}\end{array}$} & \multirow{3}{*}{ ONL } & no uveitis & $115.53[108.11,124.16]$ & $99.20[89.18,105.66]$ & $92.44[83.58,97.56]$ & $97.53[86.02,102.44]$ \\
\hline & & uveitis normal b-IT & $117.11[105.54,126.93]$ & $97.31[84.84,107.96]$ & $91.62[77.42,98.02]$ & $93.94[80.00,104.91]$ \\
\hline & & prolonged b-IT & $114.15[100.79,127.75]$ & $92.88[78.62,104.73]$ & $86.14[75.37,96.14]$ & $94.59[81.83,104.61]$ \\
\hline & & $p$-value & 0.652 & 0.150 & 0.105 & 0.865 \\
\hline & \multirow{4}{*}{$\begin{array}{l}\text { IS/ } \\
\text { OS }\end{array}$} & no uveitis & $11.47[11.13,11.85]$ & $10.45[9.96,10.71]$ & $10.20[9.82,10.46]$ & $10.35[10.06,10.62]$ \\
\hline & & uveitis normal b-IT & $11.29[10.86,11.81]$ & $10.42[10.07,10.88]$ & $10.16[9.81,10.69]$ & $10.40[10.00,10.79]$ \\
\hline & & prolonged b-IT & $11.18[10.64,12.29]$ & $10.68[10.03,12.03]$ & $10.39[9.73,11.58]$ & $10.59[10.02,11.62]$ \\
\hline & & $p$-value & 0.167 & 0.058 & 0.137 & 0.049 \\
\hline & \multirow{3}{*}{ OSL } & no uveitis & $20.40[19.59,21.72]$ & $14.22[12.07,17.73]$ & $12.93[11.47,16.62]$ & $13.94[12.53,16.75]$ \\
\hline & & uveitis normal b-IT & $20.75[18.96,22.44]$ & $14.99[13.00,18.78]$ & $14.66[12.39,19.19]$ & $15.10[12.91,18.55]$ \\
\hline & & prolonged b-IT & $21.03[19.28,23.80]$ & $17.03[14.35,20.50]$ & $16.85[13.28,20.63]$ & $16.88[13.56,20.07]$ \\
\hline & & $p$-value & 0.337 & $<0.001$ & 0.001 & 0.013 \\
\hline & & no uveitis & $20.53[17.85,22.60]$ & $21.69[16.00,24.06]$ & $22.60[16.84,24.40]$ & $21.78[17.92,24.27]$ \\
\hline & OPP & uveitis normal b-IT & $19.59[16.52,21.84]$ & $20.41[15.66,23.30]$ & $20.51[14.96,23.65]$ & $19.94[15.86,22.67]$ \\
\hline & U & prolonged b-IT & $15.73[12.05,19.20]$ & $14.09[11.06,18.73]$ & $14.34[11.96,19.03]$ & $15.18[11.88,19.22]$ \\
\hline & & $p$-value & $<0.001$ & $<0.001$ & $<0.001$ & $<0.001$ \\
\hline & & no uveitis & $14.87[14.73,15.11]$ & $14.96[14.68,15.09]$ & $14.88[14.66,15.07]$ & $14.93[14.73,15.12]$ \\
\hline u & RPE & uveitis normal b-IT & $14.85[14.52,15.09]$ & $14.84[14.59,15.07]$ & $14.80[14.59,15.01]$ & $14.74[14.48,15.05]$ \\
\hline & & prolonged b-IT & $14.79[14.42,15.03]$ & $14.86[14.52,15.06]$ & $14.82[14.60,15.00]$ & $14.75[14.53,15.00]$ \\
\hline & & $p$-value & 0.222 & 0.456 & 0.540 & 0.077 \\
\hline
\end{tabular}

Results of the mean thickness analysis of the RNFL, GCL, IPL, INL, OPL, ONL, IS/OS junction, OSL, OPR and RPE of eyes with no uveitis ( $n=39$; these eyes were of patients with a unilateral uveitis and all had a normal cone $b$-IT), uveitis eyes with a prolonged cone $b$-IT ( $n=142$ eyes), and uveitis eyes with a normal cone $b$-IT ( $n=191$ eyes). Results are presented per region of the Early Treatment Diabetic Retinopathy Study (ETDRS) grid using the lowa Reference Algorithms. Data are presented as median [IQR]. Significances were tested with Kruskal-Wallis test. All tests were two-tailed. Abbreviations: b-IT 


\begin{tabular}{|c|c|c|c|c|}
\hline region 5 & region 6 & region 7 & region 8 & region 9 \\
\hline $27.47[25.62,30.64]$ & $19.20[16.64,49.34]$ & $39.19[36.36,45.15]$ & $41.93[18.43,53.19]$ & $42.84[38.87,46.77]$ \\
\hline $29.74[25.44,33.67]$ & $30.71[19.15,55.08]$ & $43.83[38.31,50.71]$ & $29.58[19.05,56.39]$ & $46.95[39.94,53.84]$ \\
\hline $33.56[28.05,40.93]$ & $39.06[23.39,61.77]$ & $50.36[41.65,62.17]$ & $33.48[23.04,63.87]$ & $53.09[43.27,60.59]$ \\
\hline$<0.001$ & 0.001 & $<0.001$ & 0.040 & $<0.001$ \\
\hline $55.21[47.86,58.28]$ & $27.86[26.40,31.45]$ & $26.34[23.44,28.23]$ & $30.51[27.14,32.70]$ & $24.58[21.79,26.78]$ \\
\hline $52.95[45.70,58.23]$ & $30.28[26.12,34.26]$ & $25.17[22.75,30.21]$ & $29.25[25.54,34.32]$ & $23.92[20.66,27.23]$ \\
\hline $49.26[36.74,57.43]$ & $29.13[23.99,34.52]$ & $25.09[20.32,28.43]$ & $29.98[25.05,36.06]$ & $22.81[19.87,25.97]$ \\
\hline 0.026 & 0.336 & 0.168 & 0.910 & 0.220 \\
\hline $40.83[36.88,43.92]$ & $38.48[36.56,42.23]$ & $34.96[32.72,36.75]$ & $36.97[33.70,42.05]$ & $33.11[31.26,37.04]$ \\
\hline $39.19[34.23,43.31]$ & $37.82[33.97,41.39]$ & $33.89[30.42,37.37]$ & $36.82[32.47,41.27]$ & $33.74[30.29,37.29]$ \\
\hline $36.59[32.80,41.26]$ & $34.43[31.59,37.86]$ & $32.26[28.76,35.35]$ & $34.62[30.01,38.08]$ & $31.43[28.34,34.77]$ \\
\hline 0.003 & $<0.001$ & 0.001 & 0.002 & 0.001 \\
\hline $40.83[37.40,43.62]$ & $30.04[28.52,32.11]$ & $29.06[26.75,31.13]$ & $31.35[29.82,32.79]$ & $27.52[25.97,29.87]$ \\
\hline $41.68[38.41,44.97]$ & $32.23[29.45,35.14]$ & $30.09[27.77,33.05]$ & $31.92[29.29,35.30]$ & $29.26[27.10,32.16]$ \\
\hline $43.45[38.63,48.94]$ & $32.09[28.48,36.15]$ & $30.00[26.82,33.74]$ & $32.16[28.64,37.64]$ & $29.66[25.99,33.31]$ \\
\hline 0.014 & 0.042 & 0.117 & 0.364 & 0.068 \\
\hline $27.93[26.33,30.90]$ & $25.66[24.02,27.29]$ & $24.78[23.39,26.82]$ & $26.51[24.70,28.87]$ & $24.26[23.01,25.51]$ \\
\hline $27.67[25.12,31.63]$ & $26.22[24.09,28.45]$ & $24.88[23.15,26.70]$ & $26.10[23.92,29.02]$ & $24.67[22.93,26.64]$ \\
\hline $27.42[24.77,34.04]$ & $24.81[23.20,28.54]$ & $24.55[22.55,27.21]$ & $25.82[23.72,28.53]$ & $24.50[22.60,27.79]$ \\
\hline 0.925 & 0.071 & 0.486 & 0.330 & 0.636 \\
\hline $92.46[83.78,98.11]$ & $79.12[69.81,83.61]$ & $82.14[76.35,85.62]$ & $79.66[70.51,84.33]$ & $73.86[68.66,77.67]$ \\
\hline 90.15 [77.87, 98.95] & $77.06[69.44,84.68]$ & $79.84[73.28,86.61]$ & $76.05[67.83,83.81]$ & $71.71[65.09,78.00]$ \\
\hline $86.60[73.77,95.27]$ & $75.68[67.08,83.72]$ & $77.58[71.40,84.24]$ & $75.70[69.43,82.61]$ & $70.33[64.48,77.02]$ \\
\hline 0.044 & 0.382 & 0.064 & 0.357 & 0.221 \\
\hline $10.08[9.84,10.48]$ & $10.10[9.68,10.60]$ & $9.85[9.66,10.24]$ & $9.87[9.53,10.16]$ & $10.12[9.64,11.32]$ \\
\hline $10.24[9.88,10.71]$ & $10.31[9.86,11.41]$ & $10.00[9.60,10.67]$ & $10.31[9.79,11.08]$ & $10.72[9.91,12.83]$ \\
\hline $10.44[9.82,12.03]$ & $11.15[10.06,13.80]$ & $10.47[9.95,12.30]$ & $10.93[10.16,13.31]$ & $11.73[10.15,14.23]$ \\
\hline 0.042 & $<0.001$ & $<0.001$ & $<0.001$ & $<0.001$ \\
\hline $13.45[11.76,16.45]$ & $13.99[10.88,17.18]$ & $15.81[10.66,18.86]$ & $13.43[10.64,18.16]$ & $16.83[12.41,19.13]$ \\
\hline $14.49[12.34,18.03]$ & $15.49[12.24,19.16]$ & $16.12[12.08,20.28]$ & $15.52[12.67,19.16]$ & $16.78[13.73,19.40]$ \\
\hline $15.96[13.39,19.50]$ & $16.16[13.39,19.04]$ & $16.90[13.76,20.43]$ & $15.84[13.51,18.97]$ & $15.70[13.48,18.25]$ \\
\hline 0.007 & 0.065 & 0.136 & 0.080 & 0.258 \\
\hline $21.61[17.15,23.64]$ & $18.70[12.91,23.08]$ & $18.98[13.62,23.40]$ & $19.50[15.02,22.81]$ & $15.25[12.64,17.71]$ \\
\hline $18.71[14.12,22.45]$ & $16.07[12.68,20.27]$ & $14.86[12.42,20.81]$ & $15.76[12.38,20.63]$ & $13.36[11.30,16.70]$ \\
\hline $13.59[10.58,17.83]$ & $12.70[10.98,16.07]$ & $13.06[11.10,17.07]$ & $13.05[11.10,15.78]$ & 11.75 [10.11, 15.21] \\
\hline$<0.001$ & $<0.001$ & $<0.001$ & $<0.001$ & $<0.001$ \\
\hline $14.83[14.71,15.06]$ & $14.92[14.55,15.18]$ & $14.84[14.68,15.16]$ & $14.85[14.56,15.05]$ & $14.79[14.54,15.02]$ \\
\hline $14.81[14.54,15.04]$ & $14.80[14.52,15.00]$ & $14.84[14.60,15.06]$ & $14.72[14.50,14.97]$ & $14.75[14.52,14.98]$ \\
\hline $14.79[14.45,15.04]$ & $14.77[14.57,15.00]$ & $14.86[14.64,15.11]$ & $14.75[14.52,15.05]$ & $14.79[14.57,15.04]$ \\
\hline 0.439 & 0.669 & 0.634 & 0.543 & 0.600 \\
\hline \multicolumn{5}{|c|}{$\begin{array}{l}=\text { implicit time of the cone b-wave on electroretinogram, RNFL = Retinal Nerve Fiber Layer, GCL = ganglion } \\
\text { cell layer, IPL = inner plexiform layer, INL = inner nuclear layer, OPL = outer plexiform layer, ONL = outer } \\
\text { nuclear layer, IS/OS junction = inner segment outer segment junction, OSL = outer segment layer, OPR = } \\
\text { outer segment photoreceptors/retinal pigment epithelium complex, RPE = retinal pigment epithelium, IQR } \\
=\text { inter quartile range. }\end{array}$} \\
\hline
\end{tabular}


Table 3: Correlations between different flash strengths of the implicit time of the cone b-wave and mean thickness in microns of the inner plexiform layer and inner nuclear layer of the different regions of the ETDRS grid.

\begin{tabular}{|c|c|c|c|c|c|c|c|c|c|c|c|}
\hline \multicolumn{3}{|c|}{ Cone b-IT } & region 1 & region 2 & region 3 & region 4 & region 5 & region 6 & region / & region 8 & region y \\
\hline \multirow{8}{*}{ 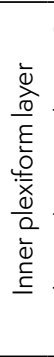 } & $0.3 \mathrm{cds} / \mathrm{m}^{2}$ & rho &, $109 *$ &,$- 185^{* *}$ &,$- 209 * *$ & $-0,101$ &,$- 203 * *$ &,$- 338 * *$ &,$- 262^{* * *}$ &,$- 261^{* * *}$ &,$- 272^{* * *}$ \\
\hline & & $p$-value & 0,036 & 0,000 & 0,000 & 0,053 & 0,000 & 0,000 & 0,000 & 0,000 & 0,000 \\
\hline & $1.0 \mathrm{cds} / \mathrm{m}^{2}$ & rho & 0,085 &,$- 184^{* * *}$ &,$- 190 * *$ & $-0,086$ &,$- 182^{* * *}$ &,$- 343^{* * *}$ &,$- 261^{* * *}$ &,$- 239 * * *$ &,$- 265^{* *}$ \\
\hline & & $p$-value & 0,103 & 0,000 & 0,000 & 0,098 & 0,000 & 0,000 & 0,000 & 0,000 & 0,000 \\
\hline & $3.0 \mathrm{cds} / \mathrm{m}^{2}$ & rho & 0,043 &,$- 265^{* * *}$ &,$- 258^{* * *}$ &,$- 159 \%$ &,$- 246 * *$ &,$- 388 * *$ &,$- 296 * *$ &,$- 296 * *$ &,$- 296^{* * *}$ \\
\hline & & $p$-value & 0,409 & 0,000 & 0,000 & 0,002 & 0,000 & 0,000 & 0,000 & 0,000 & 0,000 \\
\hline & $10.0 \mathrm{cds} / \mathrm{m}^{2}$ & rho &, $115^{*}$ &,$- 158 * *$ &,$- 214^{* * *}$ &,$- 125^{*}$ &,$- 166^{* *}$ &,$- 322^{* * *}$ &,$- 272^{* * *}$ &,$- 276^{* * *}$ &,$- 267^{* * *}$ \\
\hline & & $p$-value & 0,027 & 0,002 & 0,000 & 0,016 & 0,001 & 0,000 & 0,000 & 0,000 & 0,000 \\
\hline \multirow{8}{*}{ 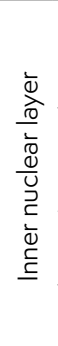 } & $0.3 \mathrm{cds} / \mathrm{m}^{2}$ & rho & $313 * *$ & $257^{* * *}$ &, $150 \% *$ &, $196 * *$ &, $141^{* * *}$ & 0,064 & 0,017 & 0,040 & 0,039 \\
\hline & & $p$-value & 0,000 & 0,000 & 0,004 & 0,000 & 0,006 & 0,219 & 0,751 & 0,447 & 0,457 \\
\hline & $0 \mathrm{cds} / \mathrm{m}^{2}$ & rho &, $332^{* * *}$ &, $215^{* *}$ &, $113^{*}$ & $204^{* * *}$ &, $122^{*}$ & 0,028 & 0,002 & 0,030 & 0,025 \\
\hline & & $p$-value & 0,000 & 0,000 & 0,030 & 0,000 & 0,019 & 0,589 & 0,964 & 0,563 & 0,626 \\
\hline & $3.0 \mathrm{cds} / \mathrm{m}^{2}$ & rho & $274^{* * *}$ & $204^{* * *}$ & 0,087 &, $148^{\mathrm{k*}}$ & 0,0764 & 0,006 & $-0,020$ & 0,004 & 0,003 \\
\hline & & $p$-value & 0,000 & 0,000 & 0,095 & 0,004 & 0,141 & 0,905 & 0,697 & 0,943 & 0,954 \\
\hline & $10.0 \mathrm{cds} / \mathrm{m}^{2}$ & rho & $301^{* * *}$ & $228 * *$ &, $131^{*}$ &, $175^{\mathrm{k*}}$ & ,130* & 0,045 & 0,014 & 0,025 & 0,028 \\
\hline & & $p$-value & 0,000 & 0,000 & 0,011 & 0,001 & 0,012 & 0,386 & 0,794 & 0,631 & 0,586 \\
\hline
\end{tabular}

Abbreviations: cone b-IT = implicit time of the cone b-wave, ETDRS = Early Treatment Diabetic Retinopathy Study.

Table 4: Correlations between clinical parameters and mean thickness in microns of the inner plexiform layer and inner nuclear layer of the different regions of the ETDRS grid.

\begin{tabular}{|c|c|c|c|c|c|c|c|c|c|c|c|}
\hline & \multicolumn{2}{|c|}{ Clinical parameters } & region 1 & region 2 & region 3 & region 4 & region 5 & region 6 & region 7 & region 8 & region 9 \\
\hline \multirow{8}{*}{ 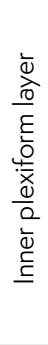 } & \multirow{4}{*}{$\begin{array}{l}\text { Cells AC } \\
\text { Vitritis }\end{array}$} & rho & $-0,038$ & 0,003 & 0,002 & $-0,005$ & $-0,030$ & $-0,010$ & 0,013 & $-0,012$ & $-0,009$ \\
\hline & & $p$-value & 0,469 & 0,950 & 0,972 & 0,929 & 0,561 & 0,845 & 0,806 & 0,818 & 0,868 \\
\hline & & rho &, $124^{*}$ & 0,100 & 0,073 &, $106^{*}$ & 0,086 & 0,019 & 0,092 & 0,086 & 0,070 \\
\hline & & $p$-value & 0,018 & 0,058 & 0,165 & 0,044 & 0,100 & 0,722 & 0,079 & 0,101 & 0,183 \\
\hline & \multirow{4}{*}{$\begin{array}{l}\text { FA score } \\
\text { Age }\end{array}$} & rho & 0,010 &,$- 187^{* * *}$ &,$- 141^{*}$ & $-0,094$ &,$- 178 * *$ &,$- 194 * *$ &,$- 119 *$ & $-0,090$ & $-0,087$ \\
\hline & & $p$-value & 0,857 & 0,001 & 0,012 & 0,098 & 0,002 & 0,001 & 0,035 & 0,111 & 0,123 \\
\hline & & rho & 0,093 &,$- 228 * *$ &,$- 311^{* * *}$ &,$- 196 * *$ &,$- 283^{* *}$ &,$- 380 * *$ &,$- 390 * *$ &,$- 375^{* * *}$ &,$- 371^{* * *}$ \\
\hline & & $p$-value & 0,073 & 0,000 & 0,000 & 0,000 & 0,000 & 0,000 & 0,000 & 0,000 & 0,000 \\
\hline \multirow{8}{*}{ 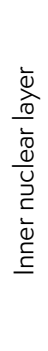 } & \multirow[t]{2}{*}{ Cells AC } & rho &, $116 *$ & ,129* &, $120^{*}$ &, $127^{*}$ &, $123^{*}$ & , 108* &, $115^{*}$ & 0,065 &, $106^{*}$ \\
\hline & & $p$-value & 0,027 & 0,013 & 0,022 & 0,015 & 0,019 & 0,040 & 0,029 & 0,218 & 0,042 \\
\hline & \multirow{4}{*}{$\begin{array}{l}\text { Vitritis } \\
\text { FA score }\end{array}$} & rho & $164^{* * *}$ & $193 * *$ &, $161^{1 * *}$ &, $124^{*}$ &, $174^{* * *}$ &, $116^{*}$ & 0,097 & ,108* & ,117* \\
\hline & & $p$-value & 0,002 & 0,000 & 0,002 & 0,018 & 0,001 & 0,027 & 0,065 & 0,039 & 0,026 \\
\hline & & rho & $207^{* * *}$ &, $222^{* * *}$ &, $166^{* * *}$ &, $151^{1 \% *}$ &, $151^{* * *}$ &, $148 * *$ &, $120 *$ &, $148 * *$ &, $156^{* * *}$ \\
\hline & & $p$-value & 0,000 & 0,000 & 0,003 & 0,007 & 0,008 & 0,009 & 0,034 & 0,009 & 0,006 \\
\hline & \multirow[t]{2}{*}{ Age } & rho & ,268** &, $226 *$ & ,108* &, $175^{* * *}$ & ,122* & $-0,061$ &,$- 173^{* * *}$ &,$- 110^{*}$ &,$- 127^{*}$ \\
\hline & & $p$-value & 0,000 & 0,000 & 0,037 & 0,001 & 0,019 & 0,244 & 0,001 & 0,034 & 0,014 \\
\hline
\end{tabular}

Abbreviations: cone b-IT = implicit time of the cone b-wave, ETDRS = Early Treatment Diabetic Retinopathy Study, AC = anterior chamber, FA = fluorescein angiography. 


\section{DISCUSSION}

This study illustrates an association between an abnormal retinal function as measured by ERG, and a difference in retinal thickness on OCT. We observed that eyes with a prolonged cone $b$-IT, had differences in thickness in the retinal layers which correspond to the bipolar cells, i.e. a thinner IPL and a thicker INL.

However, the correlation between the implicit times and thickness was weak and other factors, such as age and uveitis activity, were also correlated to the observed differences in thickness. Therefore, the prolonged cone b-IT is probably multifactorial and a change in thickness can only partly explain the observed differences. This study is to our knowledge the first that investigated the thickness of specific retinal layers in uveitis in relation to retinal function as measured by the ERG.

The thickening of the INL in uveitis could be due to subtle intraretinal edema or inflammatory deposits. One can speculate that this impairs phototransmission and therefore results in a prolonged cone b-IT on the ERG. The thinner IPL may indicate a loss of cells, which may lead to permanent damage and possibly even retinal atrophy.

Changes in retinal thickness in eyes with a prolonged cone b-IT were also observed in other layers than those which contain the bipolar cells. Except for the OPR, most layers were thickened. This is contrary to many retinal dystrophies where a loss of function is associated with a thinning of retinal layers, indicating a loss of cells. ${ }^{16}$

In line with previous reports we observed a weak and mostly negative correlation between age and retinal layer thickness. ${ }^{17,18}$ Interestingly, in the INL the correlation coefficient was positive for the inner regions, but negative for the outer regions. This indicates that for the inner regions of the INL, age may be a confounding factor in the current study. Age also affects implicit time, but differences in age cannot fully explain the observed prolonged cone b-IT because uveitis affects the implicit time more than age alone. ${ }^{5}$

Future research is necessary to investigate if anatomical or functional changes can be first observed in uveitis. To answer this question, OCT and ERG measurements have to be repeatedly done from the very first start of uveitis symptoms. Preferably, cone ERGs should be recorded with an extended protocol, because the prolonged cone b-IT is often best detected at lower stimulus strengths than at the standard light adapted 3.0 stimulus flash. ${ }^{5}$

Several studies compared the thickness of retinal layers during the active phase of uveitis to the thickness in the inactive phase, but these did not investigate the ERG. Because a prolonged cone b-IT is associated with a more active uveitis, ${ }^{5,6,19}$ some comparisons with literature can be made. 
Yamamoto et al. described that in Vogt-Koyanagi-Harada uveitis the RNFL and the inner retina (RNFL, GCL and IPL combined) were significantly thicker in the active stage of the disease. ${ }^{20}$ The correlation between an active uveitis and a thicker RNFL has also been described in several other studies, and even in uveitis eyes with glaucoma. ${ }^{21-24}$ In the current study, eyes with a prolonged cone b-IT had also a thicker RNFL compared to eyes with a normal cone b-IT. In our study vitritis and FA score were also correlated to the RNFL (data not shown).

The combination of a thinner IPL and a thicker INL was not found in other studies, because these layers were not analyzed separately. When we analyzed the combined thickness of the IPL and INL, a significant difference was only observed in region 1 (thicker) and region 6 (thinner). In anterior uveitis Lee et al. also found no difference in the combined thickness of the IPL and GCL between the active and inactive phase of uveitis. ${ }^{24}$ This highlights that it is important to evaluate the retinal layers individually, because otherwise subtle changes can be overlooked.

We saw a clearer association between a prolonged cone b-IT and a thinner OPR. In birdshot uveitis, similar findings were described, i.e. birdshot eyes had a thinner photoreceptor outer segment, which was even more affected in eyes with an abnormal ERG, which may be an indication of a more severe form of birdshot uveitis. ${ }^{25}$

A limitation of this study was that no comparison was made in retinal layer thickness between uveitis patients and healthy controls, because we do not have a database with normal values for the thickness of every retinal layer. Non-uveitis eyes of patients with unilateral uveitis are less suitable for comparison, because it may be difficult to be absolutely certain that subtle subclinical changes are absent. However, analysis of these non-uveitis eyes showed that the biggest differences in retinal thickness were between non-uveitis eyes and uveitis eyes with a prolonged cone b-IT.

Another limitation of this study was that OCT scans were analyzed using cube data. These have a lower resolution than high definition scans. However, the software program which we used only facilitates the export of thickness analysis data of scans which correspond with a 3D image. As we used a large sample size, we do not expect that a change in thickness would be in favor of one layer or the other, and therefore we expect that these results are representative.

Besides the quality of the scans, it is also important to note that the ERG measures the response of the entire retina, whereas the OCT only evaluates the central retina of approximately $6 \times 6 \mathrm{~mm}$. This should be kept in mind when imaging results are investigated in relation to functional data. Therefore, the thickening of the INL might be of less consequence on the ERG, because it was only observed in the central regions of the ETDRS grid and the macula contributes only minimally to the fullfield ERG. ${ }^{26}$ Contrary to the INL, the thinning of the IPL was present in the peripheral 
regions on the ETDRS grid. If this thinning continues towards periphery, it may be of much greater influence on the cone b-IT.

In summary, we have shown a correlation between structural and functional changes in uveitis. The observed changes in the INL and IPL can help in our understanding of the possible causes of a prolonged cone b-IT on ERG. However, the observed correlation was weak, and other clinical factors including age and uveitis activity showed similar weak correlations to the thickness of retinal layers. Thus, there is a relation between function and structure, but a prolonged cone b-IT cannot be fully explained by differences in retinal thickness on OCT alone. Because the ERG investigates the retina in a different manner than the OCT and the observed correlations were weak, there may be an additional role for the ERG in the understanding of the effects of uveitis on the retina. Further research is necessary to evaluate if changes occur first on the ERG or on OCT, in order to assess if the ERG can be used for prognosis and treatment decisions in uveitis.

\section{$\underline{\text { ACKNOWLEDGEMENTS }}$}

We would like to thank all patients for participating in the study. The authors were supported by the following foundations: Dr. F.P Fischer Stichting, and Bartiméus Fonds that contributed through UitZicht (the Netherlands). The funding organizations had no role in the design or conduct of this research. They provided unrestricted grants.

A.H. Brouwer, V.J.M. Hortensius, N.H. ten Dam., R. Wijnhoven and M.M. van Genderen have no financial disclosures. G.C. de Wit is employed as a medical physicist at Bartiméus, a low vision institute in the Netherlands. Furthermore, he is a sole proprietor, with his company Optical Diagnostics (http://www.opticaldiagnostics. com) he develops and sells ophthalmic software products and does sometimes consulting work. J.H. de Boer was payed by Abbvie for lectures.

We thank J. Ossewarde-van Norel and L. Ho (University Medical Centre Utrecht), for their help in recruiting patients to participate in this research, D. Gültzau, Y. Burgers and M. Ballast (University Medical Centre Utrecht) for their help in recording the ERGs, F. Riemslag and H. Talsma (Bartiméus Diagnostic Centre for complex visual disorders, Zeist) for giving additional electrophysiological advice and S. Risseeuw (University Medical Centre Utrecht) for her statistical advice. 


\section{$\underline{\text { REFERENCES }}$}

1. Thorne JE, Suhler E, Skup M, et al. Prevalence of Noninfectious Uveitis in the United States: A Claims-Based Analysis. JAMA Ophthalmol. 2016;134(11):1237-1245. doi:10.1001/jamaophthalmol.2016.3229

2. Suttorp-Schulten MS, Rothova A. The possible impact of uveitis in blindness: a literature survey. Br J Ophthalmol. 1996;80(9):844-848.

3. Dick AD, Tundia N, Sorg R, et al. Risk of Ocular Complications in Patients with Noninfectious Intermediate Uveitis, Posterior Uveitis, or Panuveitis. Ophthalmology. 2016;123(3):655-662. doi:10.1016/j.ophtha.2015.10.028

4. Forooghian F, Yeh S, Faia LJ, Nussenblatt RB. Uveitic foveal atrophy: clinical features and associations. Arch Ophthalmol (Chicago, III 1960). 2009;127(2):179-186. doi:10.1001/archophthalmol.2008.564

5. Brouwer AH, de Wit GC, Ten Dam NH, Wijnhoven R, van Genderen MM, de Boer $\mathrm{JH}$. Prolonged cone b-wave on electroretinography is associated with severity of inflammation in non-infectious uveitis. Am J Ophthalmol. Published online June 4, 2019. doi:10.1016/j.ajo.2019.05.028

6. Brouwer $\mathrm{AH}$, de Wit GC, ten Dam-van Loon NH, Wijnhoven R, van Genderen MM, de Boer $\mathrm{H}$. Electroretinogram abnormalities in non-infectious uveitis often persist. Acta Ophthalmol. 2020;Mar 19 Onl.

7. Sonka M, Abràmoff MD. Quantitative analysis of retinal OCT. Med Image Anal. 2016;33:165-169. doi:10.1016/j.media.2016.06.001

8. Abramoff MD, Garvin MK, Sonka M. Retinal Imaging and Image Analysis. IEEE Rev Biomed Eng. 2010;3:169-208. doi:10.1109/RBME.2010.2084567

9. Bogunović $\mathrm{H}, \mathrm{Kwon} \mathrm{YH}$, Rashid A, et al. Relationships of retinal structure and humphrey 24-2 visual field thresholds in patients with glaucoma. Invest Ophthalmol Vis Sci. 2014;56(1):259-271. doi:10.1167/iovs.14-15885

10. Sohn EH, van Dijk HW, Jiao C, et al. Retinal neurodegeneration may precede microvascular changes characteristic of diabetic retinopathy in diabetes mellitus. Proc Natl Acad Sci U S A. 2016;113(19):E2655-64. doi:10.1073/pnas.1522014113

11. McCulloch DL, Marmor MF, Brigell MG, et al. ISCEV Standard for full-field clinical electroretinography (2015 update). Doc Ophthalmol. 2015;130(1):1-12. doi:10.1007/ s10633-014-9473-7

12. Garvin MK, Abràmoff MD, Wu X, Russell SR, Burns TL, Sonka M. Automated 3-D intraretinal layer segmentation of macular spectral-domain optical coherence tomography images. IEEE Trans Med Imaging. 2009;28(9):1436-1447. doi:10.1109/ TMI.2009.2016958

13. Antony B, Abramoff MD, Tang L, et al. Automated 3-D method for the correction of axial artifacts in spectral-domain optical coherence tomography images. Biomed Opt Express. 2011;2(8):2403-2416. doi:10.1364/BOE.2.002403

14. Tugal-Tutkun I, Herbort CP, Khairallah M. Scoring of dual fluorescein and ICG inflammatory angiographic signs for the grading of posterior segment inflammation (dual fluorescein and ICG angiographic scoring system for uveitis). Int Ophthalmol. 2010;30(5):539-552. doi:10.1007/s10792-008-9263-x

15. Jabs D a. Standardization of uveitis nomenclature for reporting clinical data. Results of the first international workshop. Am J Ophthalmol. 2005;140(3):509-516. doi:10.1016/j. ajo.2005.03.057 
16. Liu G, Liu X, Li H, Du Q, Wang F. Optical Coherence Tomographic Analysis of Retina in Retinitis Pigmentosa Patients. Ophthalmic Res. 2016;56(3):111-122. doi:10.1159/000445063

17. Huo YJ, Guo Y, Li L, et al. Age-related changes in and determinants of macular ganglion cell-inner plexiform layer thickness in normal Chinese adults. Clin Experiment Ophthalmol. 2018;46(4):400-406. doi:10.1111/ceo.13067

18. Nieves-Moreno M, Martínez-de-la-Casa JM, Morales-Fernández L, Sánchez-Jean R, Sáenz-Francés F, García-Feijoó J. Impacts of age and sex on retinal layer thicknesses measured by spectral domain optical coherence tomography with Spectralis. PLoS One. 2018;13(3):e0194169. doi:10.1371/journal.pone.0194169

19. Brouwer $A H$, van Genderen MM, de Wit GC, de Boer JH. Electroretinogram abnormalities in nonanterior childhood uveitis. Acta Ophthalmol. Published online September 2018. doi:10.1111/aos.13923

20. Yamamoto M, Nishijima K, Nakamura M, Yoshimura N. Inner retinal changes in acutephase Vogt-Koyanagi-Harada disease measured by enhanced spectral domain optical coherence tomography. Jpn J Ophthalmol. 2011;55(1):1-6. doi:10.1007/s10384-0100900-3

21. Moore DB, Jaffe G), Asrani S. Retinal nerve fiber layer thickness measurements: uveitis, a major confounding factor. Ophthalmology. 2015;122(3):511-517. doi:10.1016/j. ophtha.2014.09.008

22. Bellocq D, Maucort-Boulch D, Kodjikian L, Denis P. Correlation in retinal nerve fibre layer thickness in uveitis and healthy eyes using scanning laser polarimetry and optical coherence tomography. Br J Ophthalmol. 2017;101(3):309-315. doi:10.1136/ bjophthalmol-2016-308539

23. Din NM, Taylor SRJ, Isa $\mathrm{H}$, et al. Evaluation of retinal nerve fiber layer thickness in eyes with hypertensive uveitis. JAMA Ophthalmol. 2014;132(7):859-865. doi:10.1001/ jamaophthalmol.2014.404

24. Lee M-W, Lee T-H, Won Y-K, Shin Y-I, Kim J-Y. Characteristics of retinal layer thickness in acute anterior uveitis: an optical coherence tomography study. Acta Ophthalmol.

Published online September 23, 2019. doi:10.1111/aos.14221

25. Symes R, Young M, Forooghian F. Quantitative Assessment of Retinal Degeneration in Birdshot Chorioretinopathy Using Optical Coherence Tomography. Ophthalmic Surg Lasers Imaging Retina. 2015;46(10):1009-1012. doi:10.3928/23258160-20151027-04

26. Robson AG, Nilsson J, Li S, et al. ISCEV guide to visual electrodiagnostic procedures. Doc Ophthalmol. 2018;136(1):1-26. doi:10.1007/s10633-017-9621-y 


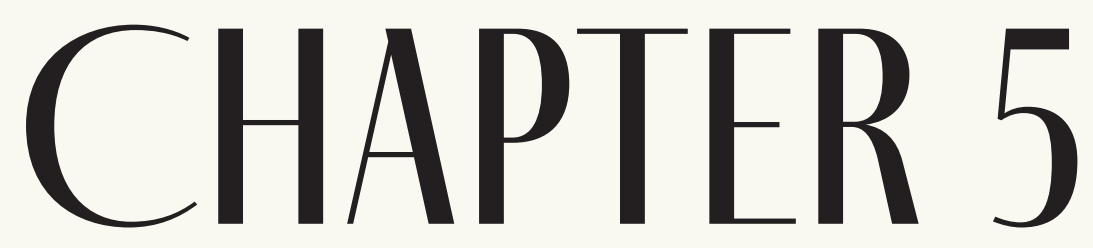

ELECTRORETINOGRAM ABNORMALITIES IN NON-ANTERIOR CHILDHOOD UVEITIS

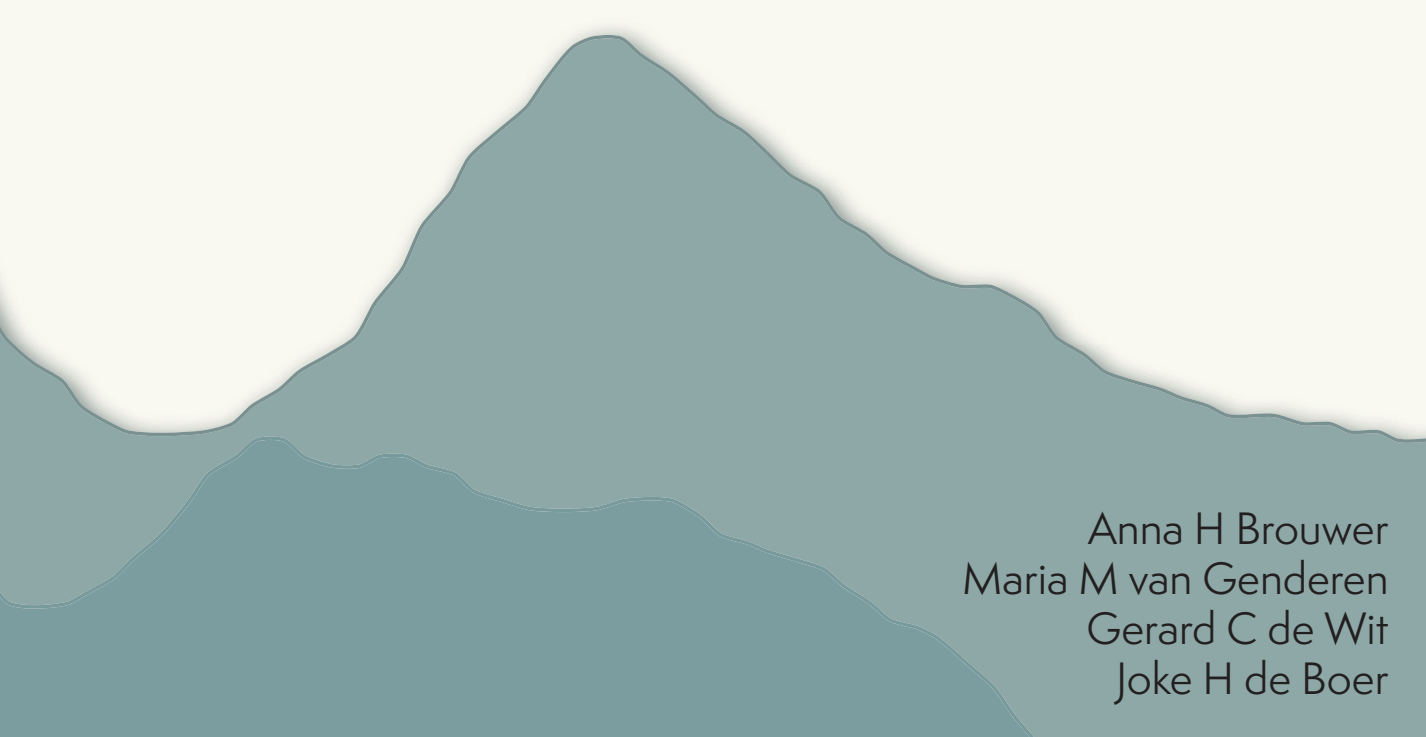

Acta Ophthalmol. 2019, doi: 10.1111/aos.13923 


\section{ABSTRACT}

\section{Purpose}

A major point of concern in uveitis is the development of irreversible retinal changes after inflammation. In this study, we assess how non-anterior childhood uveitis affects retinal function using full field electroretinography (ERG).

\section{Methods}

Cross-sectional study. ERGs of 63 uveitis eyes (33 children) were measured according to extended International Society for Clinical Electrophysiology of Vision (ISCEV) protocols. ERG abnormalities were investigated in relation to the following clinical parameters: demographics, uveitis characteristics, including severity of inflammation, treatment, best corrected visual acuity (BCVA), cystoid macular edema (CME) on optical coherence tomography (OCT) and fluorescein angiography score.

\section{Results}

The ERG showed abnormalities in 34 eyes (54\%). The most frequent ERG abnormalities were prolonged implicit times of the cone $b$-wave $(37 \% ; n=23 / 63)$ and an abnormal $30 \mathrm{~Hz}$ flicker response (implicit time and/or amplitude) (33\%; $n=21 / 63)$. Factors associated with these ERG abnormalities were CME $(p=.021)$ and 3+ vitreous cells $(p=.021)$. BCVA in eyes with and without these ERG abnormalities did not statistically differ, and was relatively good (median 0.05 LogMAR, IQR 0.00 - 0.15 LogMAR).

\section{Conclusion}

The ERG is frequently affected in childhood uveitis indicating a global retinal dysfunction. ERG abnormalities seem to be associated with a more severe posterior segment inflammation and a younger age. If an association between ERG abnormalities and long term visual outcome can be made in the future, these early ERG findings during the course of childhood uveitis have significance for treatment strategies. 


\section{$\underline{\text { INTRODUCTION }}$}

Non-anterior, non-infectious uveitis is a serious disease with unilateral blindness developing in up to $19 \%$ of children, despite intensive immunomodulating treatment. This blindness is often caused by complications such as cystoid macular edema (CME) and glaucoma. ${ }^{1-3}$

In addition, after a prolonged course of inflammation, patients with uveitis may develop thinning of the retina with "retinal dystrophy-like" changes and dragged disk vessels. ${ }^{3-5}$

To gain a better understanding of the effects of uveitis on retinal function, a full field (Ganzfield) electroretinogram (ERG) can be used. The ERG objectively measures retinal function and may therefore provide useful additional information to imaging techniques.

Electroretinography abnormalities have been described in various uveitis entities. Particularly in birdshot chorioretinopathy (BSCR), 5.6 the ERG is used for monitoring disease activity and treatment, but it may also be useful in other uveitis entities. $.7,8$ However, there are few studies on ERG abnormalities in childhood uveitis and knowledge is still lacking on the effects of uveitis on retinal function in children. ${ }^{9}$

In this study, we retrospectively analyzed ERG abnormalities in children with a non-infectious and non-anterior uveitis. We correlate their ERG abnormalities to clinical parameters and investigate the value of the ERG as an additional tool to objectively assess retinal damage in childhood uveitis.

\section{METHODS}

\section{Study population}

We included 33 patients (63 eyes) with a non-infectious, non-anterior uveitis. The median age at diagnosis was 8.9 years (range 3.5 - 14.6 years). All patients were seen at the ophthalmology department of the University Medical Centre Utrecht (UMC Utrecht). Here we perform an ERG as part of the clinical work-up if no obvious underlying cause for uveitis is found, to exclude a retinal dystrophy. ${ }^{10}$ In case of doubt regarding the ERG abnormalities, such as reduction of amplitudes, DNA was tested for retinal dystrophy mutations, which was negative $(N=3)$. Furthermore, none of the patients had alterations suggestive of a retinal dystrophy on their latest optical coherence tomography (OCT) and/or visual fields. All patients had an ERG examination between May 2015 and December 2016. 
The uveitis diagnosis was based on the Standardization of Uveitis Nomenclature (SUN) criteria" and made by an ophthalmologist specialized in uveitis. Electroretinograms and their medical charts were retrospectively reviewed.

This study was conducted in compliance with the ethical principles of the declaration of Helsinki. Ethical approval was requested and obtained from the Medical Ethical Research Committee of the UMC Utrecht. Depending on the age of the patients, we obtained consent from the patients themselves, or their parents, or both.

\section{ERG analysis}

ERGs were measured incorporating the International Society for Clinical Electrophysiology of Vision (ISCEV) standards. ${ }^{12}$ Dawson-Trick-Litzkow (DTL) electrodes were used as corneal electrodes. An Espion E3 system with ColorDome Stimulator (Diagnosys LLC, Cambridge, UK) was used for flash stimulation.

We measured an extended ISCEV series, consisting of stimulus strengths that increase with approximately $0.5 \mathrm{log}$ units and range from $0.0001-30.0 \mathrm{cds} / \mathrm{m}^{2}$ for the dark-adapted ERG (DA; rod and combined rod/cone) and from $0.3-10.0 \mathrm{cds} / \mathrm{m}^{2}$ for the light-adapted ERG (LA; cone), including a $30 \mathrm{~Hz}$ flicker response (LA; cone).

The ERGs of patients were compared to healthy controls with the same age range as uveitis patients ( 5 - 22 years) ( $N=50)$. These ERGs were measured with the same equipment and protocols and provided by the Rotterdam Eye Hospital and Bartiméus (Bartiméus Diagnostic Centre for complex visual disorders, Zeist). An ERG was considered abnormal if amplitudes were below the $2.5^{\text {th }}$ percentile, or implicit times were above the $97.5^{\text {th }}$ percentile, or both.

We categorized the ERG abnormalities based on the measuring conditions ((LA $0.3-10.0 \mathrm{cds} / \mathrm{m}^{2}$ (cone response), $30 \mathrm{~Hz}$ flicker response (cone response), DA 0.0001 - $0.01 \mathrm{cds} / \mathrm{m}^{2}$ (rod response) or DA 3.0 - $30.0 \mathrm{cds} / \mathrm{m}^{2}$ (combined rod cone response)) and which part of the response was abnormal (a-wave, b-wave, amplitudes or implicit times). We did not classify DA $0.03-1.0 \mathrm{cds} / \mathrm{m}^{2}$, as stimulus strengths lay between rod and combined rod-cone responses.

All ERGs could be used for analyses. It is our experience that in a child-friendly environment, it is possible to measure an ERG properly, even in small children.

\section{Clinical parameters}

Medical records were reviewed for age, gender, medical history and age at onset of uveitis (defined as age at the date of diagnosis by a (referring) ophthalmologist). Using the outpatient visit closest to ERG measurement, with a maximum of 2.5 months, we recorded for each eye: laterality and localization of uveitis, best corrected visual acuity 
(BCVA), cell grade in the anterior chamber, cell grade in the vitreous, flare," posterior synechiae, corneal clarity, lens clarity, snowballs, snow banking, vasculitis, optic disc hyperemia or swelling, and CME, either present on OCT (Zeiss, Cirrus HD OCT 5000), or fluorescein angiography (FA).

An experienced ophthalmologist $(\mathrm{J} d \mathrm{~d})$ scored FAs using the Fluorescein angiographic scoring system of the Angiography Scoring for Uveitis Working Group (ASUWOG).13 We looked at the FA made before or up to 2.5 months after the ERG was made, for statistical analysis. If more than one FA was made, we used the FA with the highest overall score as an indicator of structural damage of previous severity of inflammation.

We recorded per patient treatment with systemic steroids, methotrexate (MTX), mycophenolate mofetil and adalimumab and we recorded per eye the frequency of administered periocular corticosteroid injections.

\section{Statistical analysis}

For statistical analysis SPSS version 21.0 (SPSS Inc., Chicago IL) was used. Elecrroretinography abnormalities were investigated in relation to clinical parameters.

We performed all analyses twice, once using all 63 uveitis eyes, and once using only one eye per patient. We performed this second analysis as an alternative for paired sampling analyses. For this second analysis, we included the eye with the worst BCVA in case of a bilateral uveitis. If BCVA was the same in both eyes, a random eye was selected per patient.

Pearson chi squared test or the likelihood ratio was applied for categorical variables, and a Student t-test or a Mann-Whitney $U$ test was applied for continuous variables. Spearman rank correlation coefficient was used to find possible correlations between variables. We considered $p$-values below 0.05 as statistically significant. All tests were 2-tailed.

\section{$\underline{\text { RESULTS }}$}

\section{Patient characteristics}

Most patients (91\%; 30 out of 33 ) had a bilateral uveitis; $49 \%$ of the patients were male. Intermediate uveitis was seen in $64 \%$ of the patients and panuveitis in $36 \%$ of patients. In 3 patients uveitis was associated with Blau-syndrome, a rare hereditary disorder with a classical triad of arthritis, dermatitis and uveitis associated with a NOD2 mutation. ${ }^{14}$ In the other 30 patients no underlying cause was found. 


\section{ERG analysis}

The ERG was abnormal in $35(56 \%)$ eyes (21 patients, 2 with unilateral uveitis) (Table 1). In line with this, when looking at the specific ERG parameters as continuous variables, uveitis patients did decidedly worse than healthy controls in all cone, $30 \mathrm{~Hz}$, rod and combined rod cone responses, with the exception of the rod implicit times (Figure 1 and Supplemental figures 1 - 7).

The most frequently found ERG abnormality (24 eyes (38\%)), was a prolonged implicit time of the cone b-wave, which was most pronounced at the lower stimulus strengths $(0.3$ and $\left.1.0 \mathrm{cds} / \mathrm{m}^{2} \mathrm{LA}\right)$. In addition, responses showed abnormal waveforms with less steep slopes of both the ascending and descending limb of the b-wave (Figure 2).

The second most frequently found ERG abnormality (21 eyes, 33\%) was an abnormal $30 \mathrm{~Hz}$ flicker response, consisting of a reduction in amplitude, or a prolonged implicit time, or both (Figure 2).

In 16 eyes $(25 \%)$ both the implicit times of the cone b-wave were prolonged and the $30 \mathrm{~Hz}$ flicker response was abnormal. In 5 eyes the $30 \mathrm{~Hz}$ flicker response was abnormal, while the implicit times of the cone b-wave were within normal values, and in 8 eyes the $30 \mathrm{~Hz}$ flicker response was within normal values while the implicit times of cone b-waves were prolonged. No statistically significant differences were found in the b/a wave ratios of the cone ERG between uveitis and age matched controls.

Besides abnormalities in the cone b-wave and $30 \mathrm{~Hz}$ responses, abnormalities in the $\operatorname{rod}\left(0.0001-0.01 \mathrm{cds} / \mathrm{m}^{2} \mathrm{DA}\right)$ and combined rod cone $\left(3.0-30.0 \mathrm{cds} / \mathrm{m}^{2} \mathrm{DA}\right)$ responses were found in 8 eyes $(13 \%)$ and 15 eyes $(24 \%)$, respectively. Six eyes $(10 \%)$ only had abnormalities in the dark-adapted ERG, without ERG abnormalities in the light-adapted ERG.
Table 1: Electroretinogram abnormalities of uveitis eyes $(n=63)$

\begin{tabular}{ll|c} 
& \multicolumn{1}{|c}{$\begin{array}{c}\text { Eyes with ERG } \\
\text { abnormalities } \\
\text { N (\%) }\end{array}$} \\
\hline Overall & $35(56)$ \\
Single flash cone & $27(43)$ \\
A-wave $\quad$ Amplitude & $4(6)$ \\
& $\quad$ Implicit time & $2(3)$ \\
B-wave $\quad$ Amplitude & $9(14)$ \\
& $\quad$ Implicit time & $24(38)$ \\
30 Hz flicker response & $21(33)$ \\
Through $\quad$ Implicit time & $7(11)$ \\
Peak & Amplitude & $17(27)$ \\
& Implicit time & $12(19)$ \\
Single flash rod & $8(13)$ \\
A-wave $\quad$ Implicit time & $2(3)$ \\
B-wave $\quad$ Amplitude & $6(10)$ \\
& Implicit time & 0 \\
Single flash combined rod cone & $15(24)$ \\
A-wave $\quad$ Amplitude & $11(18)$ \\
& Implicit time & $2(3)$ \\
B-wave & Amplitude & $7(11)$ \\
& Implicit time & $12)$
\end{tabular}

Abbreviations: $E R G$ = electroretinography a) light adapted $0.3-10.0 \mathrm{cds} / \mathrm{m}^{2}$; b) light adapted cone response; c) dark adapted $0.0001-0.01 \mathrm{cds} / \mathrm{m}^{2} ; \mathrm{d}$ ) dark adapted 3.0 - $30.0 \mathrm{cds} / \mathrm{m}^{2}$ 


\section{$30 \mathrm{~Hz}$ Flicker response}

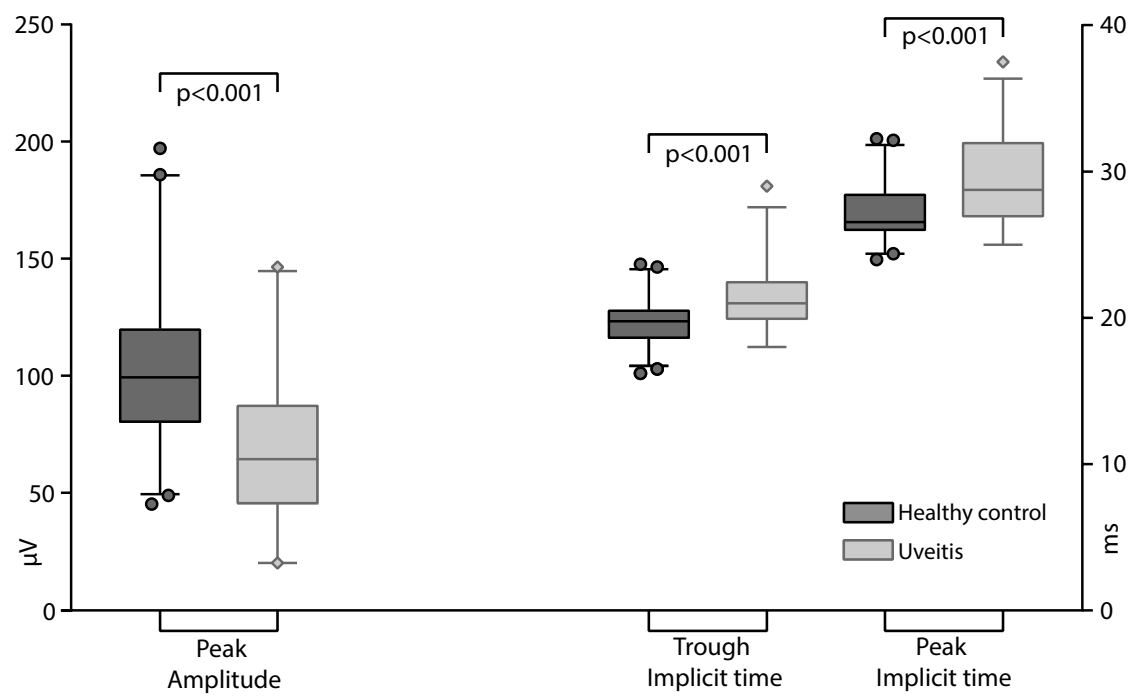

LA b-wave implicit time

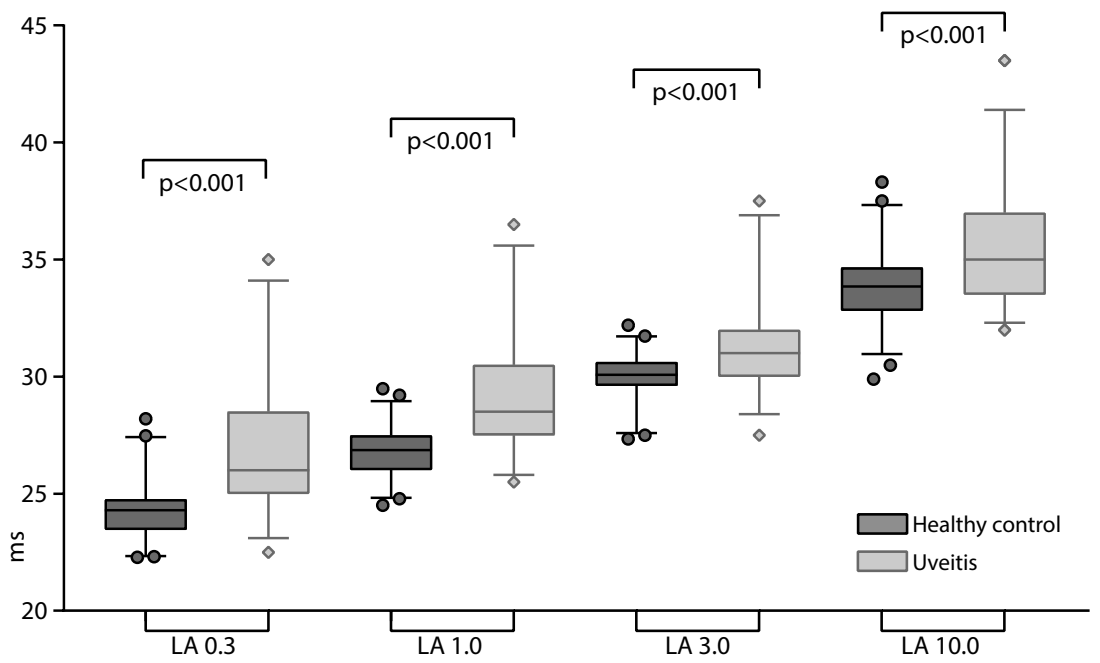

Figure 1: Boxplots of the implicit time of the cone b-wave and the $30 \mathrm{~Hz}$ flicker response of patients compared to healthy controls from the same age category (5 - 22 years, $n=50)$. For statistical analysis Mann-Whitney $U$ test was used. Abbreviations: $L A=$ light adapted. 


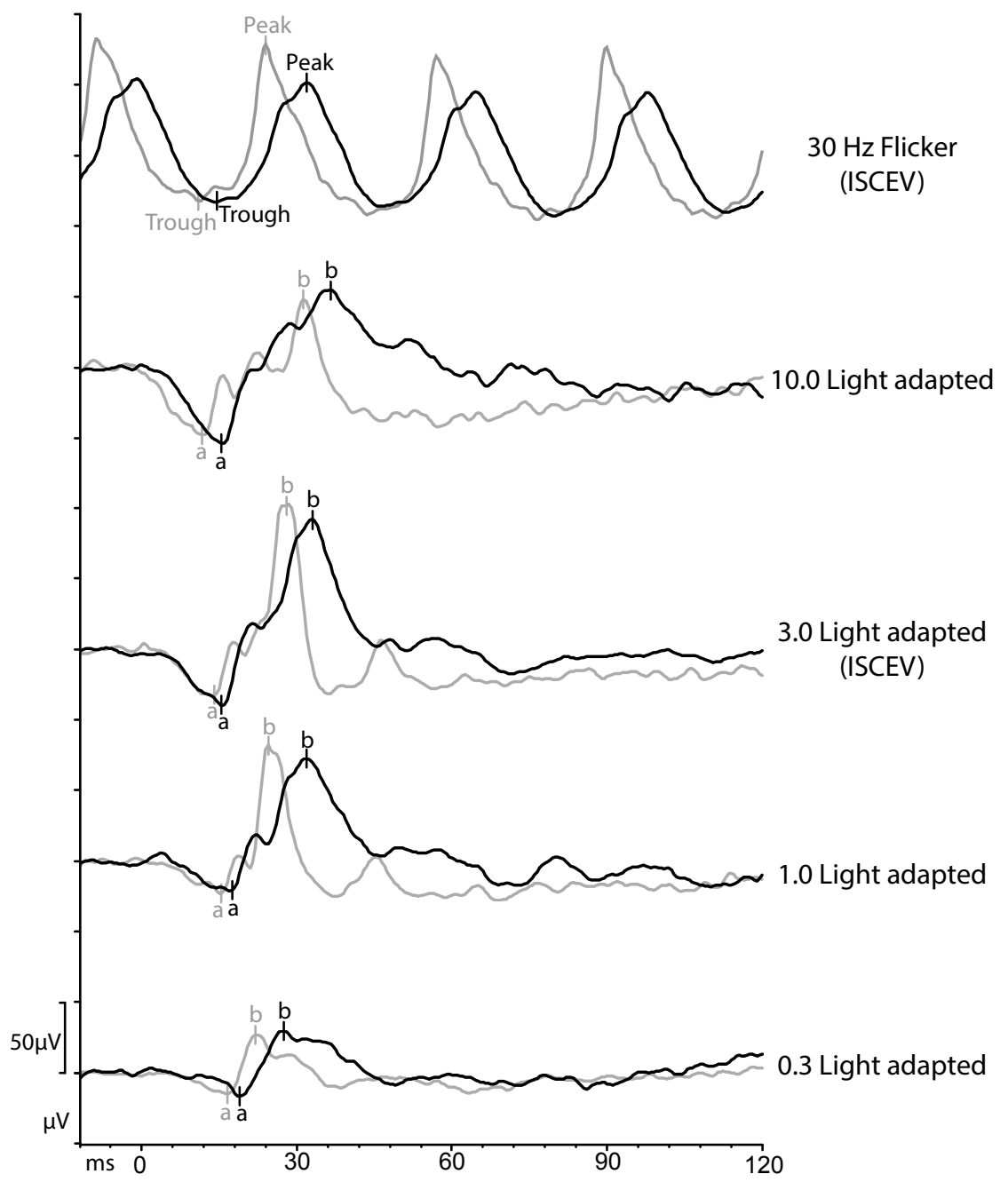

Figure 2: Representative example of a light-adapted (LA) electroretinogram (ERG) of a patient with uveitis (black) and an ERG of a age matched control (grey). The cone b-wave implicit times of the uveitis patient are prolonged; this is most pronounced at the lower stimulus strengths (light adapted 0.3 and $1.0 \mathrm{cds} / \mathrm{m}^{2}$ ). The 30 $\mathrm{Hz}$ flicker response of the uveitis patient is abnormal as well, with reduced amplitudes and prolonged implicit times.

\section{Statistical analysis of ERG abnormalities in relation to clinical parameters}

We analyzed if clinical parameters were significantly different between uveitis eyes with and without ERG abnormalities. Here we classified the ERG as abnormal if the implicit time of the cone b-wave was prolonged, or the $30 \mathrm{~Hz}$ flicker response was abnormal, or both (Table 2). We used these ERG abnormalities, since they had a typical appearance, occurred frequently and are both cone mediated. 
Table 2. Clinical characteristics of uveitis eyes in relation to electroretinogram abnormalities

\begin{tabular}{|c|c|c|c|c|}
\hline & $\begin{array}{c}\text { Eyes with } \\
\text { ERG abnormalities } \\
n=29\end{array}$ & $\begin{array}{c}\text { Eyes without } \\
\text { ERG abnormalities } \\
n=34\end{array}$ & $\begin{array}{l}\text { P-value } \\
\text { all eyes }\end{array}$ & $\begin{array}{l}\text { p-value } \\
\text { worst } \\
\text { eye }\end{array}$ \\
\hline Male & $16(55)$ & $14(41)$ & .267 & .565 \\
\hline Age in years ${ }^{a, b}$ & $11.4(9.5-13.2)$ & $14.8(11.9-15.8)$ & $.016 *$ & .067 \\
\hline Duration of uveitis in years ${ }^{\mathrm{a}, \mathrm{b}}$ & $1.7(.7-6.4)$ & $3.4(1.5-7.3)$ & .267 & .908 \\
\hline Blau syndrome & $1(3)$ & $5(15)$ & .112 & .438 \\
\hline History of CME & $17(59)$ & $12(36)$ & .080 & .309 \\
\hline $3+$ vitreous cells $s^{\mathrm{b}, \mathrm{c}}$ & $7(24)$ & 0 & $.001^{* *}$ & $.026 * *$ \\
\hline $3+$ cells in anterior chamber ${ }^{b, c}$ & $2(7)$ & 0 & .075 & .266 \\
\hline $\mathrm{CME}^{\mathrm{b}}$ & $7(24)$ & 0 & $.001 * *$ & $.021 * *$ \\
\hline Hyperemic optic disc ${ }^{b}$ & $9(31)$ & $3(9)$ & $.023 *$ & .173 \\
\hline Vitreous haze $e^{b, c}$ & $8(28)$ & $7(21)$ & .516 & .602 \\
\hline$B C V A$ in LogMAR $a, b$ & $0.10(0.00-0.40)$ & $0.05(0.00-0.11)$ & .127 & .133 \\
\hline Oral Prednisone & $21(72)$ & $28(82)$ & .345 & .602 \\
\hline Methotrexate & $11(38)$ & $17(50)$ & .337 & .246 \\
\hline Adalimumab & $5(17)$ & $7(21)$ & .735 & .805 \\
\hline Mycofenolaatmofetil & $12(41)$ & $12(35)$ & .620 & .948 \\
\hline Peribulbar steroid injections & $15(52)$ & $15(44)$ & .547 & $>.999$ \\
\hline FA total score ${ }^{\mathrm{a}, \mathrm{d}}$ & $11.0(5.0-17.0)$ & $8.0(1.8-12.0)$ & .108 & .461 \\
\hline FA capillary leakage score $e^{a, d}$ & $5.0(0-10.0)$ & $2.0(0-5.3)$ & .148 & .265 \\
\hline FA CME & $17(65)$ & $13(43)$ & .137 & .309 \\
\hline FA vasculitiss ${ }^{a, d}$ & $16(59)$ & $19(63)$ & .752 & .466 \\
\hline FA optic disc leakage $e^{a, d}$ & $23(85)$ & $23(76)$ & .413 & .391 \\
\hline
\end{tabular}

x) ERG abnormalities are defined as prolonged implicit times of the light adapted $\left(0.3-10.0 \mathrm{cds} / \mathrm{m}^{2}\right)$ cone $b$-wave and/or abnormalities of the $30 \mathrm{~Hz}$ flicker (amplitudes/implicit times). Data are given as number (\%) unless otherwise stated. a) Data given as median (IQR). b) Present at closest outpatient visit to ERG measurement. c) As described by the Standardization of Uveitis Nomenclature classification. d) FAs with the highest score were used for calculation of FA scores as described by The Angiography Scoring for Uveitis Working Group. el Only none eye per patient was used, in case of bilateral uveitis, eyes with the worst BCVA were selected, when BCVA was the same, eyes were selected at random. "significant in one of two analysis ** significant in both analyses. Abbreviations: $E R G=$ electroretinography, $N=$ number; $B C V A=$ best corrected visual acuity, LogMAR = logarithm of minimal angle of resolution, $C M E=$ cystoid macular edema, $F A=$ fluorescein angiography. 
Two clinical parameters were statistically significantly different between eyes with and without these ERG abnormalities in both the analysis using all uveitis eyes, and the analysis using only one eye per patient. These were: CME and 3+ vitreous cells (Table 2). There was no significant correlation between these two variables (Spearman's rho correlation coefficient $=0.259 ; p=0.056$ ).

No statistically significant differences were observed between the two groups with regard to treatment, or in the inflammation activity on FA score. Interestingly, we also found no statistical differences in BCVA, which was relatively good in both groups (0.05 and 0.05 LogMAR) (Table 2). Furthermore, no statistically significant differences were observed between clinical parameters and rod and combined rodcone abnormalities.

\section{$\underline{\text { DISCUSSION }}$}

In this study, more than half of the eyes with non-anterior childhood uveitis showed ERG abnormalities. The light-adapted ERG was most frequently affected, showing a prolonged cone b-wave implicit time, particularly at lower stimulus strengths, and abnormal $30 \mathrm{~Hz}$ flicker responses. Two clinical parameters were statistically significantly associated with these ERG abnormalities: CME and 3+ vitreous cells.

All eyes with CME had abnormal cone ERGs. Although cones are densely packed in the macula, ${ }^{15,16}$ macular dysfunction alone contributes only minimally to the full field cone. ${ }^{17-19}$ The abnormal ERG in the children with CME therefore indicates a global retinal dysfunction and not only macular dysfunction. ${ }^{18}$

A correlation between visual acuity and outer retinal function /represented by the a-wave) has been described, which is in line with our study in which we found a relatively good visual acuity and few a-wave abnormalities. ${ }^{20,21}$ However, a correlation between visual acuity in inner retinal function (represented by the b-wave) as found in birdshot uveitis, was not confirmed in our study on childhood uveitis. ${ }^{22}$ This discrepancy between birdshot and childhood uveitis may be due to a disease-specific inflammatory mechanism or to the duration of disease.

Besides eyes with CME, all eyes with 3+ vitreous cells also showed ERG abnormalities, indicating that more severe inflammation frequently results in retinal dysfunction.

The prolonged b-wave implicit time and the abnormal $30 \mathrm{~Hz}$ flicker response indicate an abnormal inner retinal transmission dysfunction from photoreceptors to bipolar cells. ${ }^{18}$ This abnormal signal transmission could be caused by an increased interneuronal distance secondary to an increased permeability of the blood-retinal 
barrier, caused by inflammation. ${ }^{23}$ Although the prolonged cone b-wave and the 30 $\mathrm{Hz}$ flicker response indicate inner retinal dysfunction, amplitudes were mostly normal, as were b/a wave ratios. In addition to the abnormal cone ERGs, we also found some abnormalities in the dark-adapted ERG. Although it may be interesting to investigate these abnormalities in more detail, in this study we decided to focus on the more profound and more frequent abnormalities of the cone ERG. Here we saw a consistent and recognizable pattern in the prolonged cone b-wave implicit time and the abnormal $30 \mathrm{~Hz}$ flicker response.

Our study is the first one that describes ERG changes in childhood uveitis by using an extended ISCEV based protocol, with a greater range of stimulus strengths than the ISCEV standard protocol. The abnormal timing in the cone b-wave, which we found in our study, was most profound at lower stimulus strengths $10.3 \mathrm{cds} / \mathrm{m}^{2} \mathrm{LA}$ and $\left.1.0 \mathrm{cds} / \mathrm{m}^{2} \mathrm{LA}\right)$, and therefore may not have been discovered by using the standard protocol only.

Previous reports on intermediate uveitis and childhood uveitis mostly describe abnormalities in amplitudes but rarely describe implicit times. ${ }^{24-26}$ Shamshinova et al. found a subnormal ERG response in $75 \%$ of eyes in childhood uveitis, including all anatomic subtypes. ERG abnormalities were more frequently seen when the macula was affected and in non-anterior uveitis. 9

In accordance with our findings, abnormalities of the $30 \mathrm{~Hz}$ flicker response in intermediate uveitis have been described. However, abnormal implicit times of the combined rod-cone response have also been reported, whereas we mostly found abnormal cone b-wave implicit times. ${ }^{24}$ Other studies on intermediate uveitis mainly describe differences in ERG amplitudes and do not mention implicit times. ${ }^{25,26}$

There are several limitations to this study. Due to the retrospective design and limited sample size, weak associations may not have been found. Since pediatric uveitis is not a common entity, we were unable to include more patients. Additionally, we were unable to correct for paired eyes, which would have been preferable since most patients had a bilateral uveitis. We were unable to perform a generalized estimating equation (GEE), since we had a complete separation of data in multiple variables, including CME and the amount of vitreous cells. By using only one eye per patient, we would have discarded almost half of the limited amount of data. Therefore, we decided to perform and present both the analysis with all eyes, and the analysis with only one eye per patient.

We did not find statistically significant associations with ERG abnormalities and FA scores in both analyses. We also could not correlate ERG abnormalities to visual field defects, as visual fields were only assessed in a minority of children and were often made a long time before the ERG was performed. 
Chapter 5

Considering the current findings and previous reports, we emphasize that in nonanterior childhood uveitis the global inner retinal function is frequently affected. Even though ERG abnormalities in intermediate and childhood uveitis have been reported in the past, ERG outcomes were not investigated in relation to clinical parameters as shown in our study. We recommend using an extended ISCEV protocol to detect early and subtle retinal dysfunction. If an association between ERG abnormalities and long-term visual outcome can be made in the future, these early ERG findings during the course of childhood uveitis have significance for treatment strategies. Since ERG abnormalities occur when BCVA is still relatively good, further studies should focus on the effects of this retinal dysfunction on long term visual outcome.

\section{$\underline{\text { ACKNOWLEDGEMENTS }}$}

Contributors: All the authors have contributed to the acquisition, analysis and interpretation of data and writing or revising of the manuscript. Competing Interests: All authors report no competing interests.

The author was supported by the following foundations: Dr. F.P Fischer Stichting and Vereniging Bartiméus Sonneheerdt that contributed through UitZicht. The funding organizations had no role in the design or conduct of this research. They provided unrestricted grants.

We thank all patients and/or their parents for granting permission to use their medical files for this publication. We thank F.C.C. Riemslag for his help in reviewing this article and P. Zuidhof for statistical advice. 


\section{$\underline{\text { REFERENCES }}$}

1. Kalinina Ayuso V, ten Cate HAT, van den Does P, Rothova A, de Boer JH. Young age as a risk factor for complicated course and visual outcome in intermediate uveitis in children. Br J Ophthalmol. 2011;95(5):646-651. doi:10.1136/bjo.2010.184267

2. de Boer J, Berendschot TTJM, van der Does P, Rothova A. Long-term follow-up of intermediate uveitis in children. Am J Ophthalmol. 2006;141(4):616-621. doi:10.1016/j. ajo.2005.09.035

3. Hettinga YM, Verhagen FH, van Genderen M, de Boer JH. Characteristics of childhood uveitis leading to visual impairment and blindness in the Netherlands. Acta Ophthalmol. 2014;92(8):798-804. doi:10.1111/aos.12491

4. de Smet MD, Taylor SRJ, Bodaghi B, et al. Understanding uveitis: the impact of research on visual outcomes. Prog Retin Eye Res. 2011;30(6):452-470. doi:10.1016/j. preteyeres.2011.06.005

5. Moschos MM, Gouliopoulos NS, Kalogeropoulos C. Electrophysiological examination in uveitis: a review of the literature. Clin Ophthalmol. 2014;8:199-214. doi:10.2147/OPTH.S54838

6. Tzekov R, Madow B. Visual Electrodiagnostic Testing in Birdshot Chorioretinopathy. J Ophthalmol. 2015;2015:680215. doi:10.1155/2015/680215

7. Zacks DN, Samson CM, Loewenstein J, Foster CS. Electroretinograms as an indicator of disease activity in birdshot retinochoroidopathy. Graefes Arch Clin Exp Ophthalmol. 2002;240(8):601-607. doi:10.1007/s00417-002-0506-7

8. Holder GE, Robson AG, Pavesio C, Graham EM. Electrophysiological characterisation and monitoring in the management of birdshot chorioretinopathy. Br J Ophthalmol. 2005;89(6):709-718. doi:10.1136/bjo.2004.047837

9. Shamshinova AM, Katargina LA, Orlovskaya LS. Electroretinographic findings in children with uveitis. In: Dernouchamps JP, Verougstraete C, Caspers-Velu L, Tassignon MJ, eds. Recent Advances in Uveitis. Kugler Publications, Amsterdam/ New York; 1992:397-400.

10. Hettinga YM, van Genderen MM, Wieringa W, Ossewaarde-van Norel J, de Boer JH. Retinal Dystrophy in 6 Young Patients Who Presented with Intermediate Uveitis. Ophthalmology. 2016;123(9):2043-2046. doi:10.1016/j.ophtha.2016.03.046

11. Jabs D a. Standardization of uveitis nomenclature for reporting clinical data. Results of the first international workshop. Am J Ophthalmol. 2005;140(3):509-516. doi:10.1016/j. ajo.2005.03.057

12. McCulloch DL, Marmor MF, Brigell MG, et al. ISCEV Standard for full-field clinical electroretinography (2015 update). Doc Ophthalmol. 2015;130(1):1-12. doi:10.1007/ s10633-014-9473-7

13. Tugal-Tutkun I, Herbort CP, Khairallah M. Scoring of dual fluorescein and ICG inflammatory angiographic signs for the grading of posterior segment inflammation (dual fluorescein and ICG angiographic scoring system for uveitis). Int Ophthalmol. 2010;30(5):539-552. doi:10.1007/s10792-008-9263-x

14. Sarens IL, Casteels I, Anton J, et al. Blau Syndrome-Associated Uveitis: Preliminary Results From an International Prospective Interventional Case Series. Am J Ophthalmol. 2018;187:158-166. doi:10.1016/j.ajo.2017.08.017

15. Jonas JB, Schneider U, Naumann GO. Count and density of human retinal photoreceptors. Graefes Arch Clin Exp Ophthalmol. 1992;230(6):505-510. 
16. Osterberg G. Topography of the layer of rods and cones in the human retina. Acta Ophthalmol Suppl. 1935;6:1-103.

17. Khan KN, Robson A, Mahroo OAR, et al. A clinical and molecular characterisation of CRB1-associated maculopathy. Eur J Hum Genet. 2018;26(5):687-694. doi:10.1038/ s41431-017-0082-2

18. Robson AG, Nilsson J, Li S, et al. ISCEV guide to visual electrodiagnostic procedures. Doc Ophthalmol. 2018;136(1):1-26. doi:10.1007/s10633-017-9621-y

19. Dawson WW, Maida TM. Relations between the human retinal cone and ganglion cell distribution. Ophthalmologica. 1984;188(4):216-221. doi:10.1159/000309366

20. lannetti L, Spinucci G, Abbouda A, De Geronimo D, Tortorella P, Accorinti M. Spectral-domain optical coherence tomography in uveitic macular edema: morphological features and prognostic factors. Ophthalmologica. 2012;228(1):13-18. doi:10.1159/000337234

21. Maheshwary AS, Oster SF, Yuson RMS, Cheng L, Mojana F, Freeman WR. The association between percent disruption of the photoreceptor inner segment-outer segment junction and visual acuity in diabetic macular edema. Am J Ophthalmol. 2010;150(1):63-67.e1. doi:10.1016/j.ajo.2010.01.039

22. Sobrin L, Lam BL, Liu M, Feuer WJ, Davis JL. Electroretinographic monitoring in birdshot chorioretinopathy. Am J Ophthalmol. 2005;140(1):52-64. doi:10.1016/j. ajo.2005.01.053

23. Noma H, Mimura T, Kuse M, Shimada K. Association of electroretinogram and morphological findings in central retinal vein occlusion with macular edema. Clin Ophthalmol. 2014;8:191-197. doi:10.2147/OPTH.S54546

24. Cantrill HL, Ramsay RC, Knobloch WH, Purple RL. Electrophysiologic changes in chronic pars planitis. Am J Ophthalmol. 1981;91(4):505-512.

25. Tetsuka S, Katsumi O, Mehta MC, Tetsuka H, Hirose T. Electrophysiological findings in peripheral uveitis. Ophthalmologica. 1991;203(2):89-98.

26. Ikeda H, Franchi A, Turner G, Shilling J, Graham E. Electroretinography and electrooculography to localize abnormalities in early-stage inflammatory eye disease. Doc Ophthalmol. 1989;73(4):387-394. doi:10.1007/BF00154494 


\section{SUPPLEMENTAL MATERIAL}
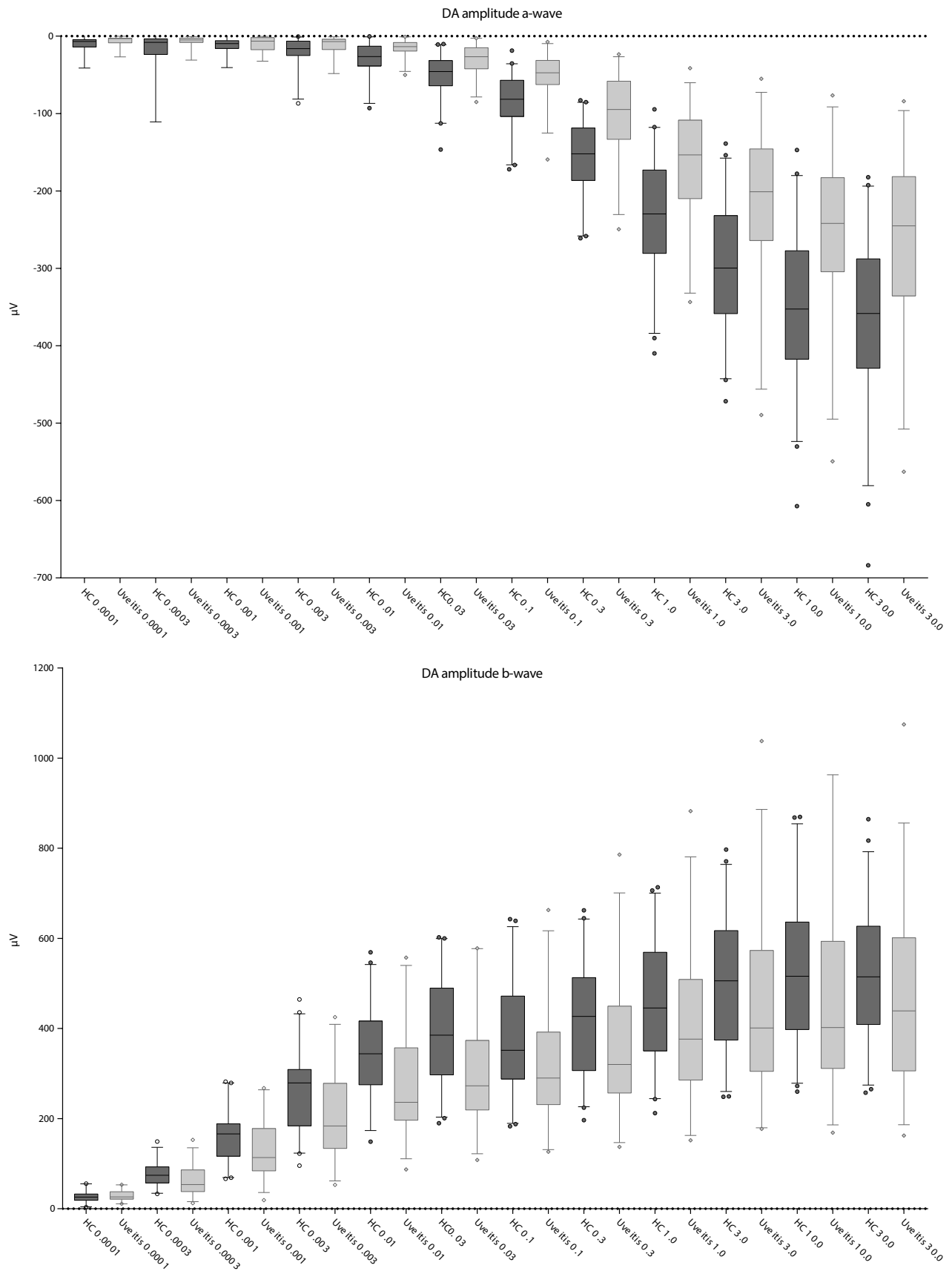

Supplemental figures 1 (top) and 2 (bottom): Boxplots of the amplitude of the rod and combined rod-cone a-wave (top) and b-wave (bottom) of patients compared to healthy controls from the same age category 15 22 years, $n=50)$. Uveitis patients has statistically significant worse outcomes for rod and combined rod-cone responses compared to healthy controls using Mann-Whitney $U$ test. Abbreviations: DA = dark adapted, HC = healthy control. 


\section{Chapter 5}
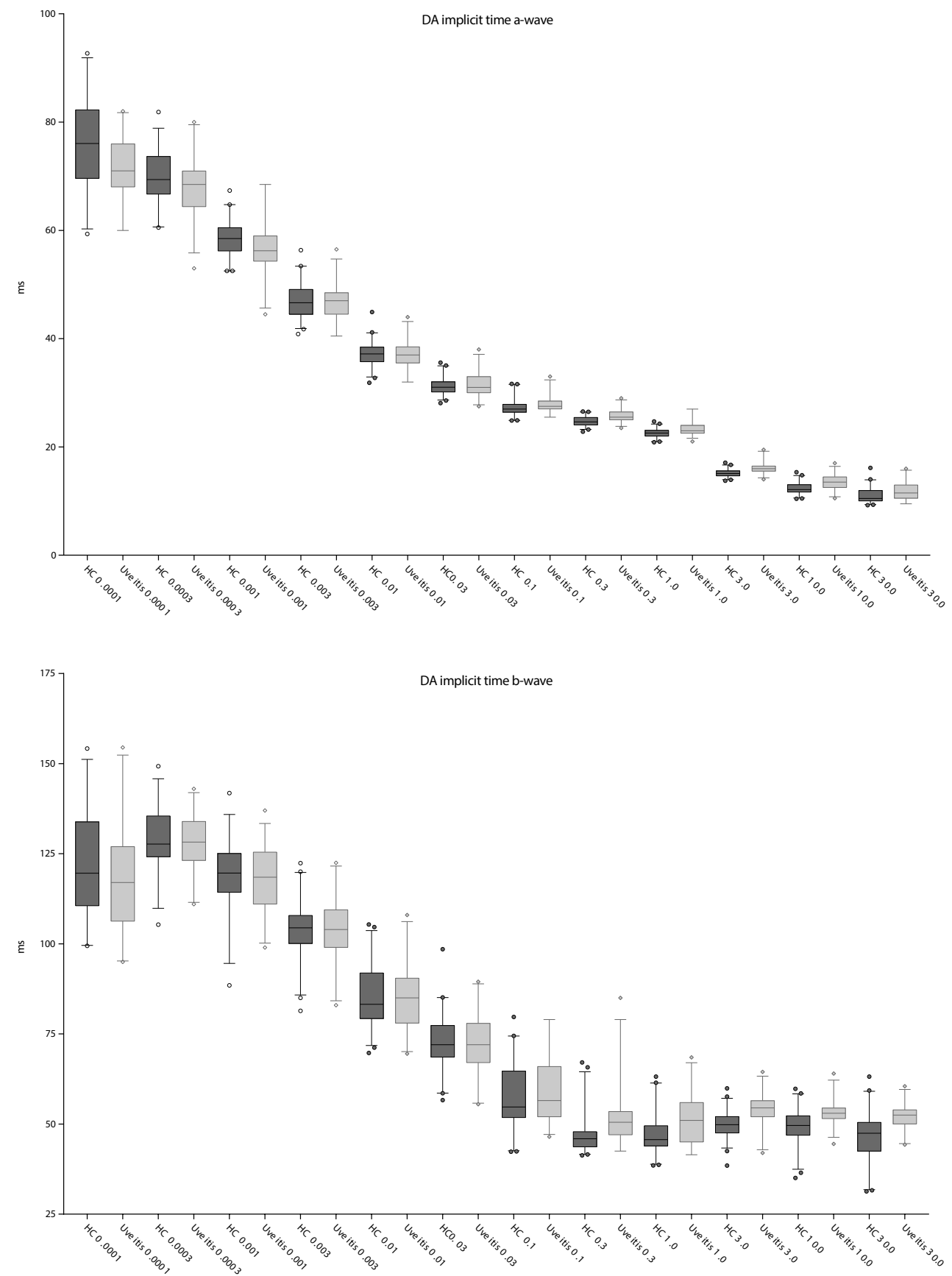

Supplemental figures 3 (top) and 4 (bottom): Boxplots of the implicit times of the rod and combined rod-cone a-wave (top) and b-wave (bottom) of patients compared to healthy controls from the same age category 15 - 22 years, $n=50)$. Uveitis patients has statistically significant worse outcomes for the combined rod-cone response compared to healthy controls using Mann-Whitney $U$ test. Abbreviations: DA = dark adapted, HC = healthy control. 


\section{LA amplitude a-wave}

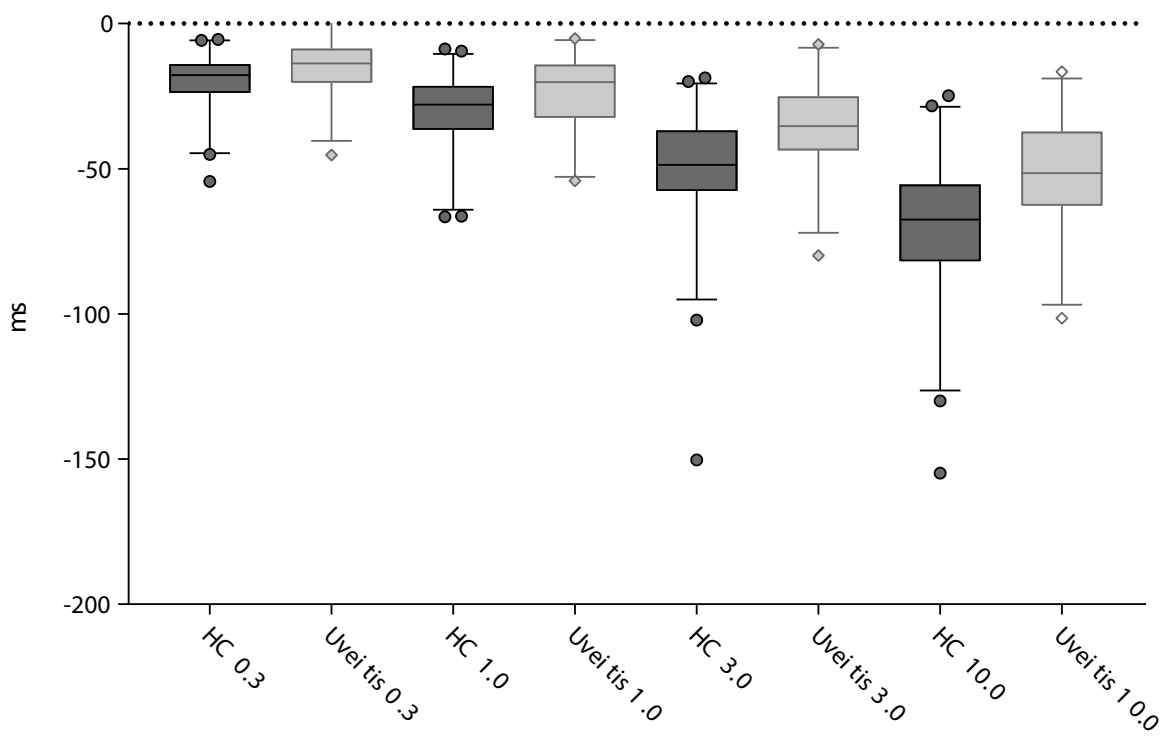

Supplemental figure 5: Boxplots of the amplitude of the cone a-wave of patients compared to healthy controls from the same age category (5 - 22 years, $n=50)$. Uveitis patients has statistically significant worse outcomes in all flash intensities compared to healthy controls using Mann-Whitney $U$ test. Abbreviations: $L A=$ light adapted, $H C=$ healthy control.

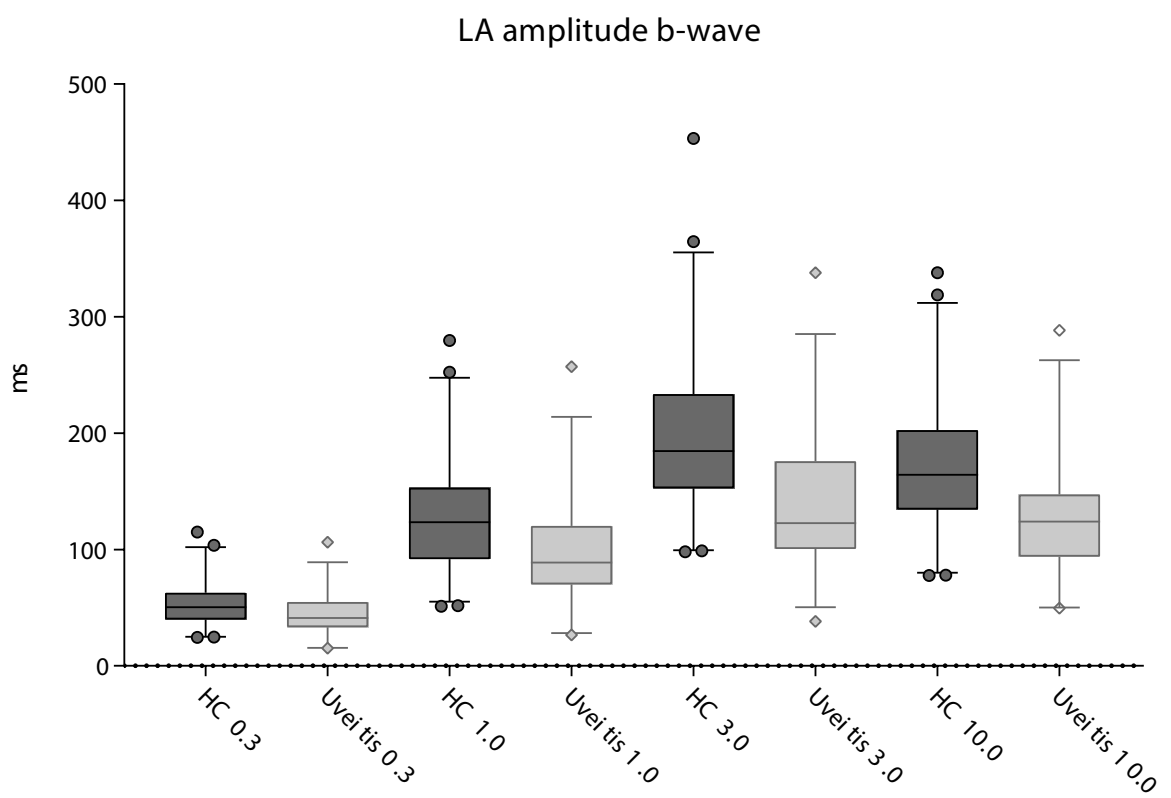

Supplemental figure 6: Boxplots of the amplitude of the cone b-wave of patients compared to healthy controls from the same age category (5 - 22 years, $n=50)$. Uveitis patients has statistically significant worse outcomes in all flash intensities compared to healthy controls using Mann-Whitney $U$ test. Abbreviations: $L A=$ light adapted, HC = healthy control. 
Chapter 5

\section{LA implicit time a-wave}

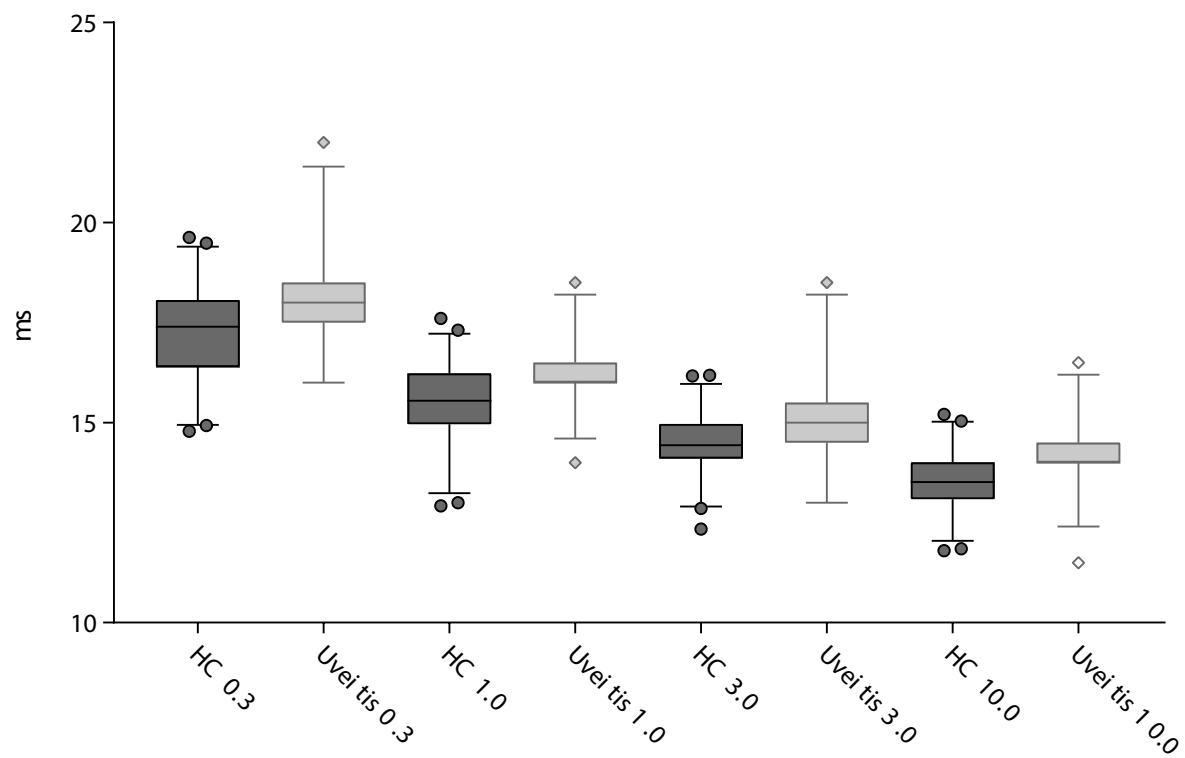

Supplemental figure 7: Boxplots of the implicit time of the cone a-wave of patients compared to healthy controls from the same age category (5 - 22 years, $n=50)$. Uveitis patients has statistically significant worse outcomes in all flash intensities compared to healthy controls using Mann-Whitney U test. Abbreviations: LA = light adapted, $H C=$ healthy control. 
ERG abnormalities in non-anterior childhood uveitis 

$\sqrt{(00)}(1)$ anconety and A) (1) Nolus 10

Niv

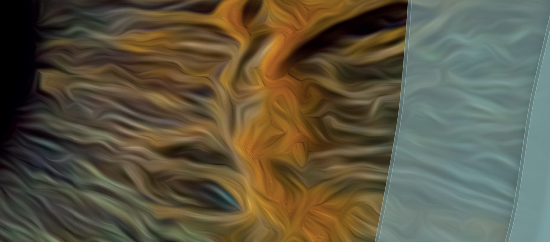

-

(

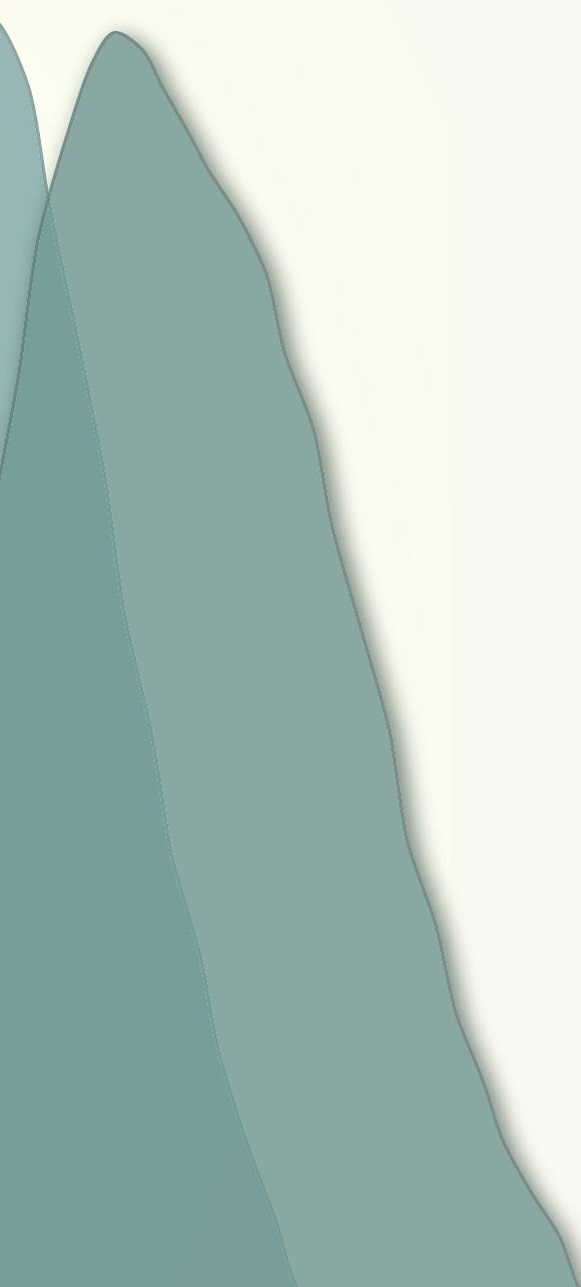

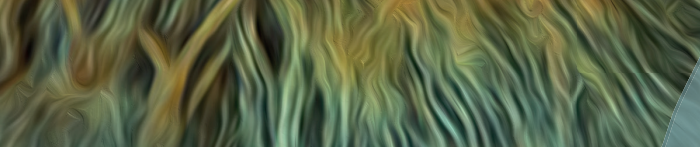
(25) (19) 


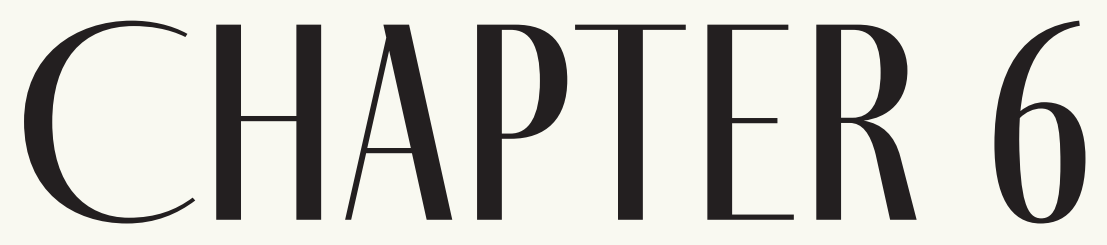

JUVENILE IDIOPATHIC ARTHRITIS UVEITIS:

A TYPICAL ANTERIOR UVEITIS, BUT THE

RETINAL FUNCTION CAN BE AFFECTED

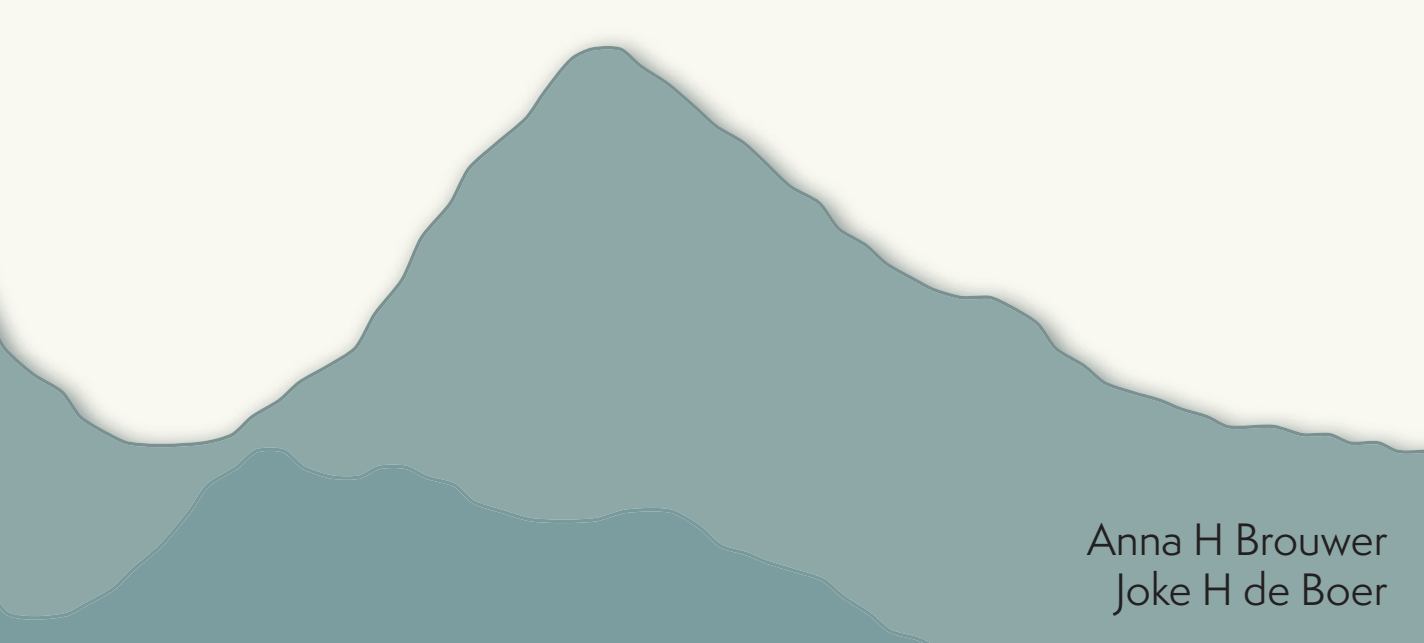


Chapter 6

\section{ABSTRACT}

\section{Purpose}

To describe the retinal function as measured by full-field electroretinogram (ERG) in patients with juvenile idiopathic arthritis (JIA) associated uveitis.

\section{Methods}

ERGs were measured according to an extended ISCEV protocol.

\section{Results}

Ten of the 16 uveitis eyes (8 patients) showed ERG abnormalities. The type of ERG abnormalities varied amongst patients and occurred in both amplitudes and implicit times, in a-waves, b-waves, $30 \mathrm{~Hz}$ flicker response and in both the dark adapted and the light adapted ERG. Five eyes had an active inflammation at the time of the ERG recording; all of these showed ERG abnormalities. Five out of eleven inactive uveitis eyes showed ERG abnormalities. No correlations were found between ERG abnormalities and the duration of uveitis, mean deviation on visual field analysis, or central macular thickness. None of the eyes had severe media opacities which could have explained the observed ERG abnormalities.

\section{Conclusion}

Even though JIA is a typical anterior uveitis, the retinal function can be affected in this type of uveitis. 
JIA uveitis: a typical anterior uveitis, but the retinal function can be affected

Dear editor,

Juvenile idiopathic arthritis (JIA) associated uveitis is typically described as a chronic anterior uveitis. We would like to present 8 patients with longstanding JIA uveitis laged between 17 - 40 years) with suspicional retinal thinning on optical coherence tomography (OCT), of whom we additionally measured a full-field electroretinogram (ERG). In 10 of the 16 uveitis eyes we observed ERG abnormalities (Figure 1). These ERG abnormalities indicate that although the inflammation in JIA is mainly located in the anterior part of the eye, the retina can be affected as well. To the best of our knowledge, this is the first report that describes that the ERG can be affected in this particular type of uveitis.

The ERGs were measured according to an extended international society for clinical electrophysiology of vision (ISCEV) protocol. This protocol incorporates the ISCEV standards, but includes more flash strengths than the standard ISCEV protocol. The flash strengths increase with approximately $0.5 \mathrm{log}$ units steps and range from $0.0001-30.0 \mathrm{cds} / \mathrm{m}^{2}$ (12 flash strengths) for the dark-adapted ERG (DA) and from 0.3 - $10.0 \mathrm{cds} / \mathrm{m}^{2}$ (4 flash strengths) for the light-adapted ERG (LA) and include a $30 \mathrm{~Hz}$ flicker response. Dawson-Trick-Litzkow (DTL) electrodes were used as corneal active electrodes, and an Espion E3 system with Colordome stimulator (Diagnosys LLC, Cambridge, UK) for flash stimulation. Our reference values were previously described.'

The type of ERG abnormalities varied among patients. Table 1 gives an overview of the ERG results. We observed abnormalities in all parts of the ERG: both in amplitudes and implicit times, in a-waves, b-waves and the $30 \mathrm{~Hz}$ flicker response. Abnormalities occurred in both the dark adapted (DA) and the light adapted (LA) ERG. The only ERG parameter which showed no abnormalities was the implicit time of the rod b-wave indicating that the rod response might be less affected than the cone response in JIA uveitis. In some eyes the amplitudes were severely diminished, whereas in others the implicit times were mainly prolonged. The most frequent ERG abnormality was a prolonged cone a-wave, which was abnormal in eight eyes. These findings highlight that different aspects of retinal function can be affected in JIA uveitis, which is in line with other forms of uveitis where ERG abnormalities may be observed in all aspects of the ERG, but the light adapted ERG is most frequently affected.'

We previously reported a characteristic type of ERG abnormality in noninfectious uveitis: a prolonged cone b-wave, which was associated with the severity of inflammation, but in these studies no patients with JIA uveitis were included.1.2 The ERGs of the current JIA patients showed a prolonged cone b-wave in 6 eyes. Recently we observed that the prolonged cone b-wave can improve in other forms of uveitis, particularly in eyes where the inflammation in the anterior chamber becomes less 

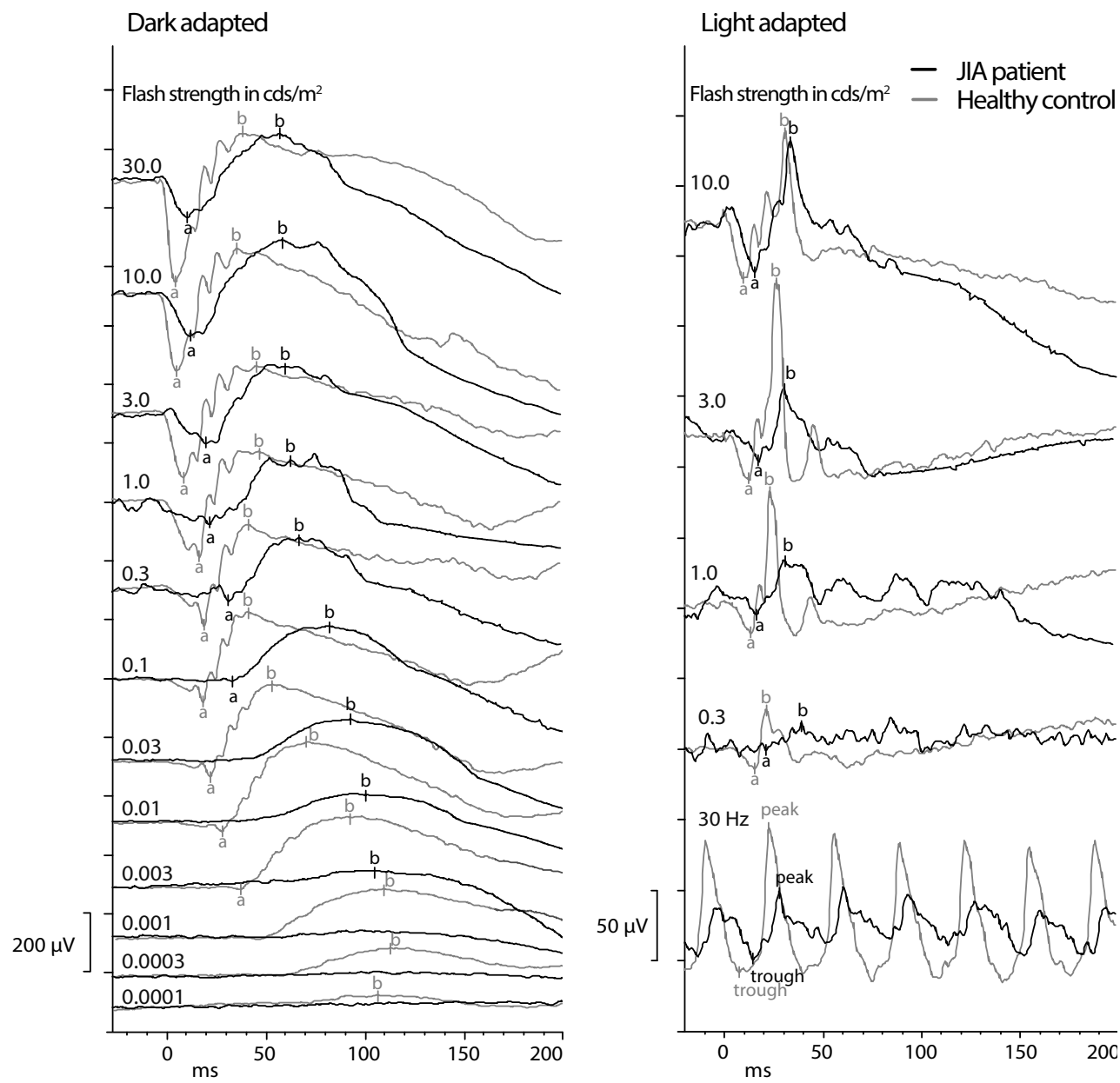

Example of ERG curves of a IIA uveitis patient (black) and an ERG of a healthy control (grey). The implicit times of a-waves and b-waves of both the dark adapted and light adapted are prolonged. Amplitudes are often reduced as well. Abbreviations: $E R G=$ electroretinogram, $J I A=$ juvenile idiopathic arthritis, $c d s / \mathrm{m}^{2}=$ candela $\cdot$ seconds/squared meters, $m s=$ milliseconds, $\mu V=$ microvolts.

active. $^{3}$ This might indicate that the observed ERG abnormalities in these JIA patients can also improve.

However, we have also observed that ERGs of uveitis patients can deteriorate over time. ${ }^{3}$ If this can also occur in JIA uveitis, should be the subject of further studies. There are reports that retinal atrophy can occur in JIA patients, usually as the result of chronic cystoid macular edema (CME), a complication of JIA uveitis., ${ }^{4,5}$ None of the JIA eyes had a retinal atrophy (central macular thickness $(C M T)<150 \mu \mathrm{m})$, but because ERG abnormalities may occur prior to retinal atrophy, we investigated the effects of CME on the ERG. We found no association between CMT and ERG abnormalities (Spearman's correlation coefficient -.267, $p=0.317$ ). At the time of the ERG recording, 
Table 1: Overview of electroretinogram abnormalities and clinical parameters of patients with juvenile idiopathic arthritis associated uveitis

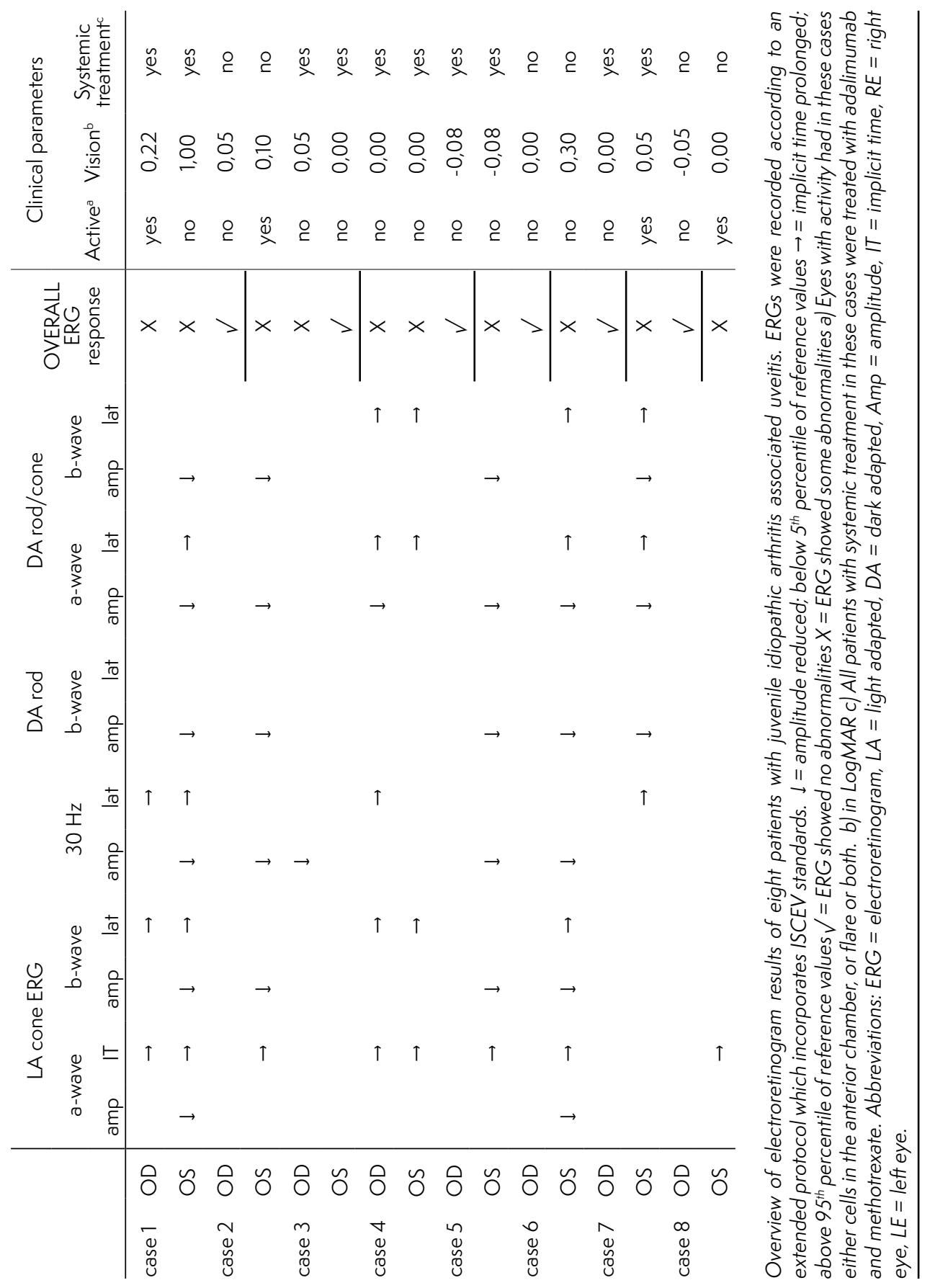


the median CMT of all eyes was $264 \mu \mathrm{m}$ (IQR 250 - 260). Three eyes of the sixteen eyes had diffuse CME (CMT) > $304 \mu \mathrm{m}$ on optical coherence tomography (OCT) at some point during their follow-up. Two eyes had a longer period of hypotony which resulted in macular folds. These folds were still present at the day of the ERG.

One patient had a history of a retinal detachment in his left eye (case 1) which was treated with a posterior vitrectomy with silicon oil. The oil was removed 5 years prior to the ERG recording. The ERGs of this patient were severely abnormal in both eyes, but as expected, the eye with a history of retinal detachment was more affected.

We did not find an association between a type of treatment and the absence or presence of ERG abnormalities. Five patients were treated with systemic medication (adalimumab and methotrexate) at the time of ERG recording and two patients were treated with only ocular steroid droplets. One patient had no medication at the time of ERG recording. This patient showed no signs of inflammation, but had an abnormal ERG in one eye, possibly due to the effects of end-stage glaucoma. These findings are in line with our previous studies were we also found no association between type of treatment and ERG abnormalities. ${ }^{1-3}$ The absence of such an association could be due to the small population, different treatment strategies, and heterogeneity in the population due to responders and non-responders to treatment.

In our previous studies we found a strong association between an active inflammation and ERG abnormalities., In the current series, five eyes showed some signs of active inflammation (trace of anterior chamber cells, flare range 1+ - 3+) at the time of ERG recording and all of them had an abnormal ERG. However, of the eleven eyes that had an inactive uveitis at the time of the ERG recording, five had an abnormal ERG.

Although ERG abnormalities can occur early in the disease process of uveitis, in the current series the disease duration was long (median 18.9 years, IQR 16.6 - 19.8). If ERG abnormalities can also be observed in patients with a short duration of uveitis in JIA, remains therefore speculative. However, we found no association between the duration of JIA uveitis and ERG abnormalities (Spearman's correlation coefficient 1.0, $p>$.999). Also, no relation was found between the mean deviation on VF and ERG abnormalities (median -3.14, IQR -4.65 - -0.80, Spearman's correlation coefficient $-.392 p=0.143$ ).

None of the patients had severe media opacities that could have influenced the ERG results. All pupils were fully dilated at the time of ERG recording and none of the eyes had posterior synechiae at the time of ERG recording. None of the patients had central band keratopathy.

With this letter we hope to illustrate to electro-physicists and ophthalmologists treating JIA uveitis that the retinal function can be affected in JIA uveitis, even though 
the primary site of inflammation in this form of uveitis is in the anterior part of the eye. Further research is needed to evaluate if there is, similar to other forms of uveitis, an associations between the activity of uveitis and ERG abnormalities in JIA uveitis. If such an association is found, ERG abnormalities could warrant an intensifying of treatment in JIA uveitis in order to prevent a permanent loss of retinal function.

\section{$\underline{\text { ACKNOWLEDGEMENTS }}$}

We would like to thank all patients and M.M. van Genderen electrophysiological advise. The authors were supported by the following foundations: Dr. F.P Fischer Stichting, and Bartiméus Fonds that contributed through UitZicht (the Netherlands). The funding organizations had no role in the design or conduct of this research. They provided unrestricted grants. This study follows the principles of the Declaration of Helsinki. All patients gave written consent. A.H. Brouwer has no financial disclosures. J.H. de Boer was payed by Abbvie for lectures. 


\section{$\underline{\text { REFERENCES }}$}

1. Brouwer AH, de Wit GC, Ten Dam NH, Wijnhoven R, van Genderen MM, de Boer $\mathrm{JH}$. Prolonged cone b-wave on electroretinography is associated with severity of inflammation in non-infectious uveitis. Am J Ophthalmol. Published online June 4, 2019. doi:10.1016/j.ajo.2019.05.028

2. Brouwer AH, van Genderen MM, de Wit GC, de Boer JH. Electroretinogram abnormalities in nonanterior childhood uveitis. Acta Ophthalmol. Published online September 2018. doi:10.1111/aos.13923

3. Brouwer $\mathrm{AH}$, de Wit GC, ten Dam-van Loon NH, Wijnhoven R, van Genderen MM, de Boer $\mathrm{H}$. Electroretinogram abnormalities in non-infectious uveitis often persist. Acta Ophthalmol. 2020;Mar 19 Onl.

4. de Boer J, Steijaert A, van den Bor R, Stellato R, Ossewaarde-van Norel J. Development of macular edema and impact on visual acuity in uveitis associated with juvenile idiopathic arthritis. Ocul Immunol Inflamm. 2015;23(1):67-73. doi:10.3109/092 73948.2013.871566

5. Ducos de Lahitte G, Terrada C, Tran THC, et al. Maculopathy in uveitis of juvenile idiopathic arthritis: an optical coherence tomography study. Br J Ophthalmol. 2008;92(1):64-69. doi:10.1136/bjo.2007.120675 
JIA uveitis: a typical anterior uveitis, but the retinal function can be affected 


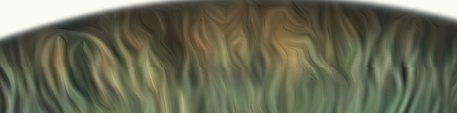
(N)

M)

Mov.

(1) 3) a) $(1)$ (1)

Now 10

(1)

(1)

)

\&

8

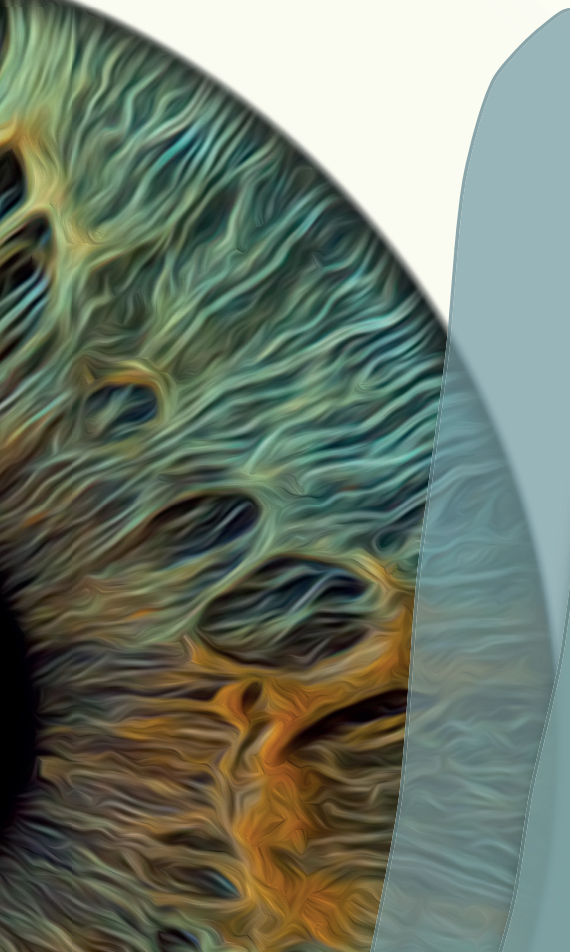

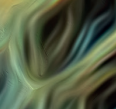
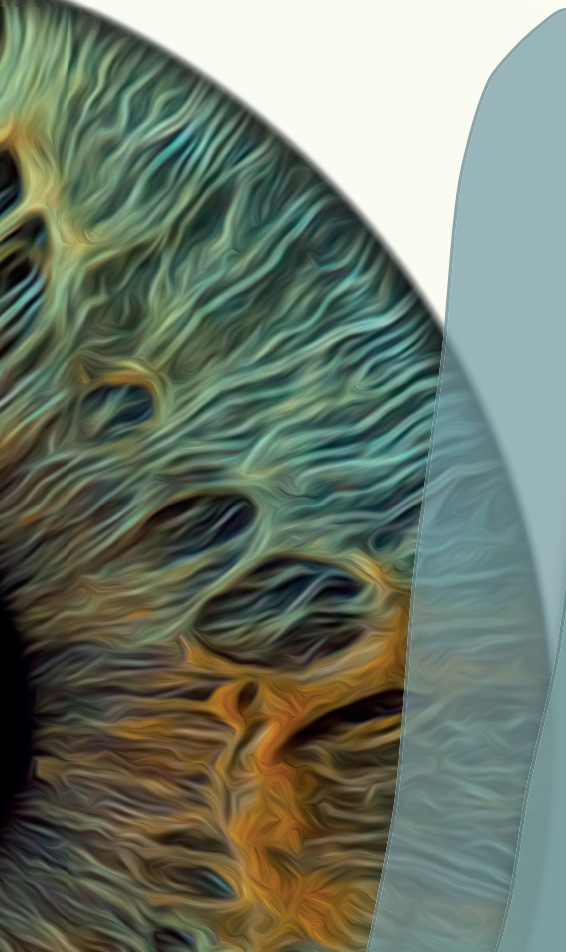

M)

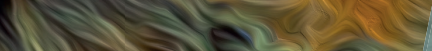

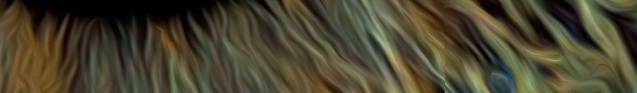

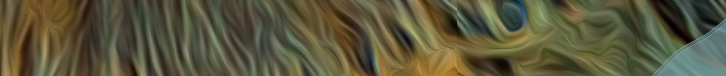

1) (No)

(1)

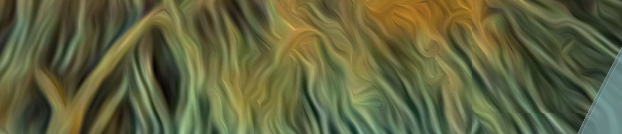

19)

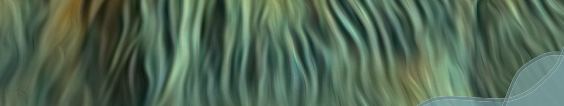

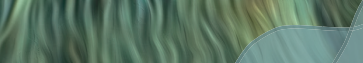

(15) 


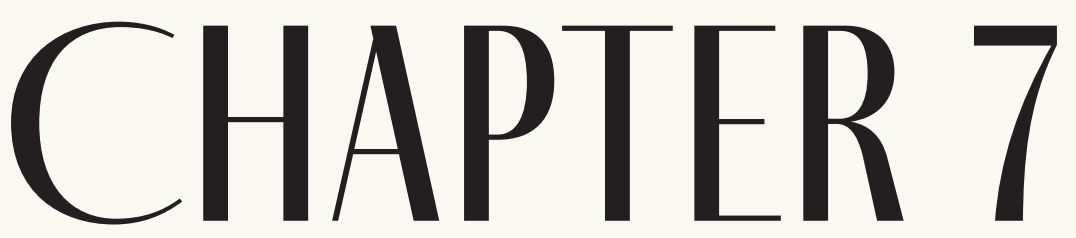

EFFECTS OF DIL ELECTRODE POSITION ON THE AMPLITUDE AND IMPLICIT TIME OF THE ELECTRORETINOGRAM

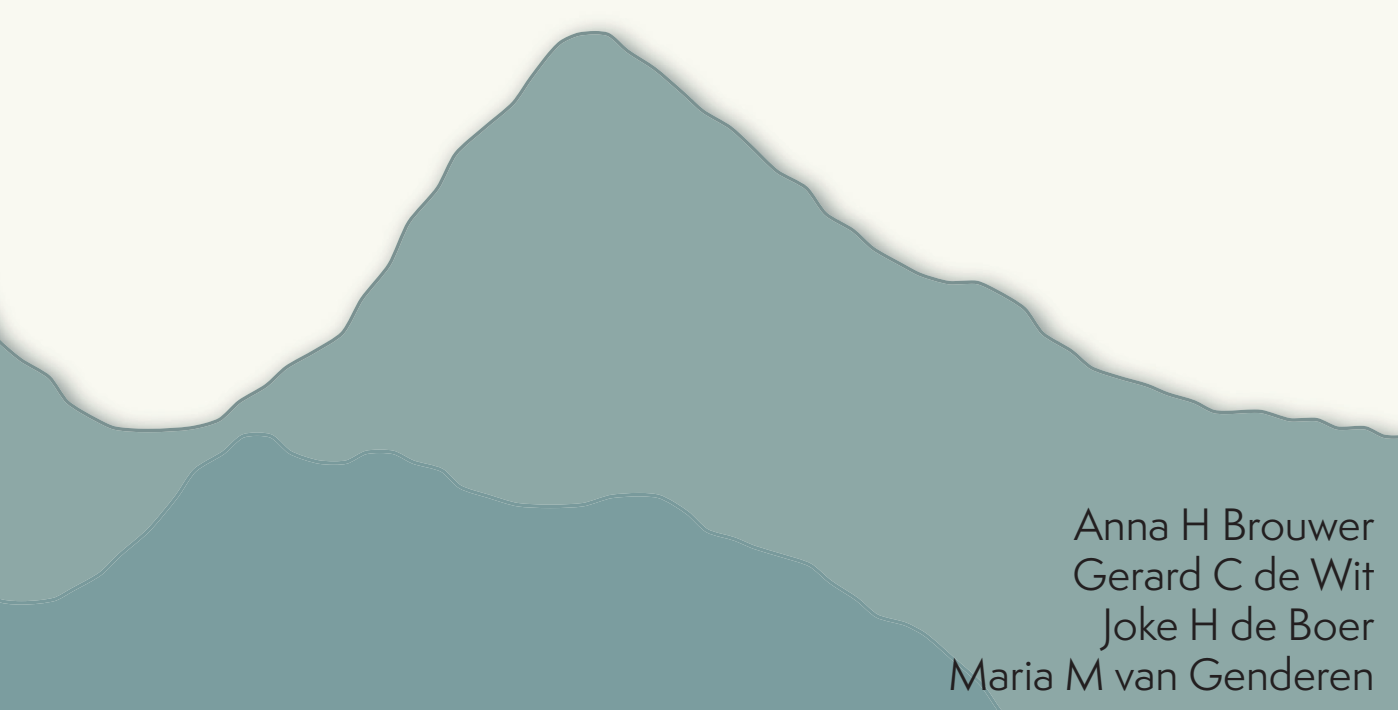

Doc Ophthalmol. 2020, doi:10.1007/s10633-019-09733-3 


\section{ABSTRACT}

\section{Purpose}

This study sought to investigate whether there is an optimal position of the Dawson, Trick, and Litzkow (DTL) electrodes when measuring the full-field electroretinogram (ERG) for monitoring purposes.

\section{Methods}

In 200 uveitis patients an extended light adapted (LA) ERG protocol was measured twice, incorporating the International Society for Clinical Electrophysiology of Vision standards. First a LA ERG was measured with the DTL in the lower lid position (LLP), and thereafter in the fornix position. Differences in amplitudes and implicit times of a-waves, b-waves and the $30 \mathrm{~Hz}$ peak were investigated. Intraclass correlation coefficients (ICC) as well as coefficients of variation (CoV) were calculated, to assess both reliability and relative variability between the two DTL positions.

\section{Results}

Implicit times showed no statistically significant differences between the two DTL positions. As expected, amplitudes at the different stimulus strengths were 1.12 - 1.19 higher in the LLP, but there were no significant differences in the CoV between the two DTL positions. The ICC was high for the b-wave and $30 \mathrm{~Hz}$ flicker response (0.842 0.979), but lower for the a-wave, especially for amplitudes (0.584 - 0.716).

\section{Conclusions}

For monitoring purposes in patients, we conclude that based on relative variability, no position is preferable above the other. However, because in most diseases amplitudes are decreased, the LLP may be chosen because it yields higher amplitudes. Whatever the choice, it is important to ensure that the DTL position remains stable during an ERG recording. 


\section{INTRODUCTION}

Since the introduction of the Dawson Trick Litzkov (DTL) electrode', its use in recording electroretinograms (ERGs) has spread. One of the main advantages of the DTL is that it is much more comfortable to wear compared to conventional electrodes, such as contact lens electrodes. ${ }^{2-4}$

Originally, the ERG was mainly used for diagnosing retinal diseases such as retinal dystrophies, where the ERG is frequently severely abnormal. However, now that the ERG is increasingly used for monitoring disease, more subtle ERG changes become important. Therefore, one must be aware of factors that may affect the ERG results, other than disease or treatment. Particularly factors that influence the intersession variability are important.

Factors that may influence the absolute ERG results, but will have little effect on inter-session variability since they do not differ between sessions, include gender, ${ }^{5}$ refraction, ${ }^{6}$ and ocular pigmentation. ${ }^{7}$ Other factors that can affect the intersessionvariability may be minimized by always incorporating International Society for Clinical Electrophysiology of Vision (ISCEV) standards. These include duration of dark- and light adaptation, flash strengths and pupil size. ${ }^{8}$ Besides these, there are factors that also affect the intersession variability but are more difficult to address. These include: DTL position, ${ }^{9-12}$ media opacities, ${ }^{13}$ and age. ${ }^{14-16}$

When the DTL electrode was introduced, it was said that its position was 'only a little disturbed by blinking'. However, since then several reports state that the DTL position can shift, which may significantly affect the recorded amplitudes.

When the DTL is positioned on the cornea, the highest amplitudes can be recorded, which decrease as the DTL is positioned more toward the fornix. ${ }^{17}$ Despite these differences in amplitudes, the latest ISCEV standard does not advice which DTL position should be used. The most common DTL positions are: the lower lid position (LLP) and the fornix position (FP). ${ }^{8}$

In our clinic, reference values are based on ERG measurements recorded at the LLP. This position was advised by the manufacturer for two reasons. First, it would ensure high amplitudes, without much risk of scratching the cornea. Second, blinking would have little effect on the DTL position. In some cases however, we have seen the DTL shift toward the fornix during an ERG recording.

Some studies advise using the FP, because it would shift less easily and therefore lead to a more stable recording. The FP would yield lower amplitudes but the ERG would be less variable. ${ }^{911}$ However, these studies were conducted in relatively small groups of healthy volunteers. These healthy volunteers are probably often coworkers who know how to cooperate during an ERG since they are familiar with ERG 
procedures. Therefore, it is useful to see if the same results can be obtained in a large cohort of patients, who may be scared or photophobic which makes the recording of an ERG more difficult. Also, a larger group can yield more reliable confidence intervals of differences between the two DTL positions.

The aim of this study was to determine the optimal DTL electrode position for monitoring purposes. We investigated whether one DTL position yields more stable ERG results than the other. If such a difference is found, this would imply that this DTL electrode position should be used for monitoring purposes. We compared the reliability as well as the relative variability of the results and investigated differences in amplitudes and implicit times.

\section{METHODS}

\section{Subjects}

The subject population, consisting of 200 patients (355 uveitis eyes, 45 unaffected eyes) with a non-infectious uveitis, aged $\geq 18$ years (median 53.4, IQR 39.2 - 63.7), has been previously described.18 All patients were mentally competent and gave their written consent to participate. This study was conducted in compliance with the ethical principles of the declaration of Helsinki. Ethical approval was requested and obtained from the Medical Ethical Research Committee of the University Medical Centre Utrecht.

\section{ERG measurement}

All ERGs were measured according to the ISCEV standards. ${ }^{8}$ An Espion E3 system with Colordome stimulator (Diagnosys LLC, Cambridge, UK) was used for full-field flash stimulation. Eyes were anesthetized with oxybuprocain $0.4 \%$. Pupils were dilated with tropicamide $0.5 \%$. Cup electrodes were used as ground and reference electrodes and placed on the forehead and on the temples. Impedances of reference and DTL electrodes were below $5 \mathrm{k} \Omega$ and below $10 \mathrm{k} \Omega$ for the ground electrode.

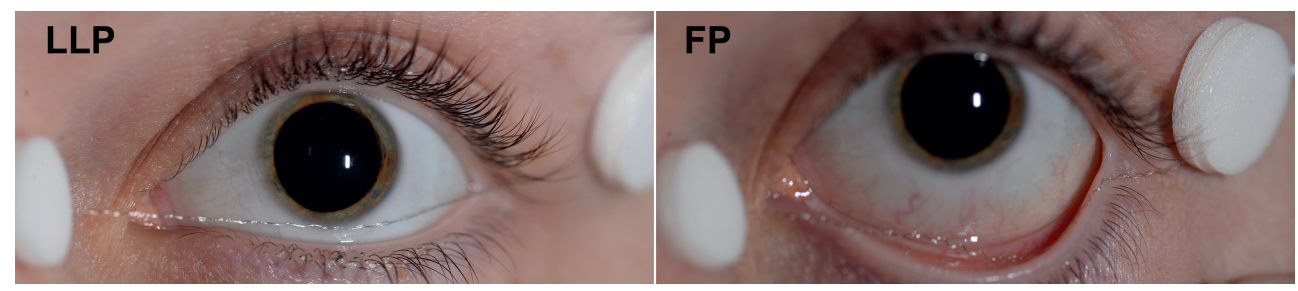

Figure 1: Representative example of the two Dawson, Trick, Litzkov (DTL) positions that were used: the lower lid position (LLP) (left) and the fornix position (FP) (right). 
An extended ISCEV protocol was measured twice, consisting of stimulus strengths that increase with approximately 0.5 log units and range from 0.3 to $10.0 \mathrm{cds} / \mathrm{m}^{2}$ for the light-adapted ERG (LA), including a $30 \mathrm{~Hz}$ flicker response $\left(\mathrm{LA}, 3.0 \mathrm{cds} / \mathrm{m}^{2}\right)$. For analyses, averages were used of two results, each consisting of five sweeps. In cases when sweeps with a trend were accepted during recording, these sweeps were toggled, or removed.

After 10 minutes of light adaptation, the first LA ERG was recorded with the DTL electrode in the LLP, and the second with the FP (see Figure 1). The DTL position was checked prior to each measurement and adjusted if necessary.

\section{Analyses}

For statistical analysis R. Studio version 1.0.143 was used. To compare differences between the two DTL positions, a Wilcoxon signed rank test was used (data not normally distributed as tested with histograms, Q-Q-plots and Shapiro-Wilk test).

We added a random value between -0.25 and $0.25 \mathrm{~ms}$ to each implicit time, because our equipment measures every $0.5 \mathrm{~ms}$. By adding this random value, the implicit time becomes a true continuous variable which has statistical advantages for calculating more reliable confidence intervals. The implicit time difference and amplitude ratio of the two DTL positions were calculated and plotted.

To evaluate the effect of the two DTL positions on the percentage of patients that would fall outside normal limits, we compared the results to our reference values. These reference values were obtained with the DTL in LLP and were previously described. ${ }^{18}$

To investigate the amount of reliability between the two measurements, intraclass correlation coefficients (ICCs) were calculated (two-way model, type consistency, unit average). For visualization of the amount of agreement between the two positions, Bland-Altman plots were made as well.

To evaluate the amount of relative variability of the ERG data at the two DTL positions, we calculated the coefficients of variation ( $\mathrm{CoV}$, sd/mean), also known as relative standard deviation. The data were transformed (square root) to fit a normal distribution. This was necessary since the results of uveitis patients can range from normal to abnormal which gives a skewed distribution. ${ }^{18}$ Normality was evaluated using Shapiro Wilk tests, histograms and Q-Q-plots. We used the R package crequality (Version 0.1.3; Marwick and Krishnamoorthy 2018) to test for significant differences in CoV. Using Bonferroni's correction, we defined p-values of $<0.006$ as statistically significant. All significances were two-tailed. 


\section{$\underline{\text { RESULTS }}$}

Table 1 shows the results of the ERG responses of the different DTL positions. Median amplitudes were significantly higher (between 1.12 and 1.19 for the different stimulus strengths, see Table 2) and amplitude ranges were wider for all responses recorded with LLP compared to the FP. In contrast, there were no significant differences in implicit times between the two DTL positions. Figure 2 shows a representative example of ERGs curves of both DTL positions obtained from the same patient.

To highlight what the effects may be of an unnoticed shift in DTL position on ERG results, we compared the results of the LLP and the FP to our reference values lobtained with the DTL in the LLP). Here, amplitudes were much more frequently

Table 1. ERG results and differences of two DTL electrode positions

\begin{tabular}{|c|c|c|c|c|c|}
\hline & & wer lid & & prnix & \\
\hline & median & {$[\mathrm{IQR}]$} & median & [IQR] & $p$-value \\
\hline A-wave amplitude* & & & & & \\
\hline 0.3 & -11.8 & {$[-15.5,-8.1]$} & -10.1 & {$[-13.0,-7.4]$} & $<0.001^{* * *}$ \\
\hline 1.0 & -19.4 & {$[-25.1,-14.3]$} & -16.3 & {$[-20.1,-11.9]$} & $<0.001^{* * * *}$ \\
\hline 3.0 & -29.7 & {$[-37.8,-22.2]$} & -24.9 & {$[-30.5,-19.8]$} & $<0.001 * * *$ \\
\hline 10.0 & -46.1 & {$[-57.2,-35.4]$} & -37.4 & {$[-45.9,-31.4]$} & $<0.001^{* * * *}$ \\
\hline A-wave implicit time & & & & & \\
\hline 0.3 & 19.0 & {$[18.2,20.0]$} & 18.9 & {$[18.0,20.1]$} & 0.445 \\
\hline 1.0 & 17.1 & {$[16.4,18.0]$} & 17.1 & {$[16.3,17.8]$} & 0.115 \\
\hline 3.0 & 15.7 & {$[15.1,16.4]$} & 15.8 & {$[15.1,16.5]$} & 0.816 \\
\hline 10.0 & 14.8 & {$[14.3,15.5]$} & 14.8 & {$[14.2,15.5]$} & 0.119 \\
\hline B-wave amplitude** & & & & & \\
\hline 0.3 & 33.3 & {$[23.3,42.0]$} & 26.2 & {$[20.4,34.6]$} & $<0.001 * * *$ \\
\hline 1.0 & 76.0 & {$[55.5,97.6]$} & 63.1 & {$[49.9,82.4]$} & $<0.001^{* \cdots *}$ \\
\hline 3.0 & 119.8 & {$[92.2,151.7]$} & 103.2 & {$[81.7,129.1]$} & $<0.001^{* * * *}$ \\
\hline 10.0 & 118.4 & {$[93.0,144.6]$} & 105.0 & {$[82.6,122.8]$} & $<0.001^{* * * *}$ \\
\hline B-wave implicit time & & & & & \\
\hline 0.3 & 27.0 & {$[25.8,30.0]$} & 27.2 & {$[25.6,29.9]$} & 0.718 \\
\hline 1.0 & 28.9 & {$[27.8,31.0]$} & 28.8 & {$[27.7,30.8]$} & 0.105 \\
\hline 3.0 & 31.6 & {$[30.7,33.0]$} & 31.6 & {$[30.7,33.0]$} & 0.129 \\
\hline 10.0 & 35.9 & {$[34.8,37.3]$} & 36.0 & {$[34.7,37.2]$} & 0.055 \\
\hline $30 \mathrm{~Hz}$ flicker Peak & & & & & \\
\hline Amplitude & 67.1 & {$[47.2,86.3]$} & 58.0 & {$[43.9,74.7]$} & $<0.001^{* * * *}$ \\
\hline Implicit time & 29.1 & {$[27.5,31.3]$} & 29.1 & {$[27.6,31.3]$} & 0.461 \\
\hline
\end{tabular}

Descriptive statistics of the ERG responses of the two DTL positions (LLP and FP). "Results of different flash strengths in candela seconds/meters squared of the light adapted ERG." Abbreviations: $E R G$ = electroretinogram, DTL = Dawson, Trick, Litzkov electrode, IQR = inter quartile range, $L L P=$ lower lid position, $F P=$ fornix position. 
Effects of DTL position on amplitude and implicit time

\begin{tabular}{|c|c|c|c|c|c|}
\hline \multirow{2}{*}{\multicolumn{2}{|c|}{$\begin{array}{l}\text { Table 2. Amplitude ratio an } \\
\text { Amplitude ratio }\end{array}$}} & \multirow[b]{2}{*}{ Median } & \multirow[b]{2}{*}[\mathrm{IQR}]{} & \multicolumn{2}{|c|}{ Percentiles } \\
\hline & & & & $2.5^{\text {th }}$ & $97.5^{\text {th }}$ \\
\hline \multirow[t]{4}{*}{ A-wave } & $0.3^{*}$ & 1.18 & {$[0.85,1.56]$} & 0.35 & 3.73 \\
\hline & $1.0^{*}$ & 1.18 & {$[0.93,1.52]$} & 0.55 & 3.06 \\
\hline & $3.0 *$ & 1.19 & {$[0.98,1.43]$} & 0.55 & 2.34 \\
\hline & $10.0^{*}$ & 1.19 & {$[1.00,1.44]$} & 0.71 & 2.11 \\
\hline \multirow[t]{4}{*}{ B-wave } & $0.3^{*}$ & 1.19 & {$[1.01,1.39]$} & 0.73 & 2.23 \\
\hline & $1.0 *$ & 1.13 & {$[1.00,1.33]$} & 0.79 & 1.80 \\
\hline & $3.0 *$ & 1.15 & {$[1.00,1.33]$} & 0.78 & 1.75 \\
\hline & $10.0^{*}$ & 1.12 & {$[1.00,1.31]$} & 0.76 & 1.79 \\
\hline \multirow{2}{*}{\multicolumn{2}{|c|}{$30 \mathrm{~Hz}$ flicker response }} & 1.12 & {$[0.97,1.31]$} & 0.73 & 1.80 \\
\hline & & & & \multicolumn{2}{|c|}{ Percentiles } \\
\hline \multicolumn{2}{|c|}{ Implicit time difference } & Mean & (SD) & $2.5^{\text {th }}$ & $97.5^{\text {th }}$ \\
\hline \multirow[t]{4}{*}{ A-wave } & $0.3^{*}$ & 0.03 & $(1.32)$ & -3.22 & 2.47 \\
\hline & $1.0^{*}$ & 0.07 & $(0.92)$ & -1.75 & 1.69 \\
\hline & $3.0^{*}$ & -0.02 & $(0.79)$ & -2.18 & 1.50 \\
\hline & $10.0^{*}$ & 0.05 & $(0.72)$ & -1.41 & 1.31 \\
\hline \multirow[t]{4}{*}{ B-wave } & $0.3^{*}$ & -0.03 & $(1.36)$ & -2.85 & 2.93 \\
\hline & $1.0 *$ & 0.02 & $(0.85)$ & -1.71 & 1.64 \\
\hline & $3.0^{*}$ & 0.04 & $(0.80)$ & -1.92 & 1.90 \\
\hline & $10.0^{*}$ & 0.05 & $(0.63)$ & -1.61 & 1.48 \\
\hline \multicolumn{2}{|c|}{$30 \mathrm{~Hz}$ flicker response } & 0.01 & $(0.85)$ & -1.73 & 1.70 \\
\hline
\end{tabular}

Results of the amplitude ratio (lower lid position/ fornix position) and implicit time difference /lower lid positionfornix position) of the different flash strengths in candela seconds/meters squared of the light adapted ERG. "candela seconds/meters squared.

defined as abnormal when the FP was used (Supplemental table 1). However, in 5\% (20 eyes) the ERG was defined as abnormal in the LLP, but normal in the FP.

Figure 3 shows the amplitude ratio LLP/FP and the implicit time difference LLP - FP, for the b-wave $\left(3.0 \mathrm{cds} / \mathrm{m}^{2}\right)$ of both uveitis eyes (circle) and unaffected eyes (square). It illustrates that in most cases the amplitude ratio is higher than 1.0 which implies that the amplitude that was measured at the LLP was higher than at the FP. In contrast to this, the implicit time difference is evenly spread around zero, which implies that there was no tendency for the implicit time to be either higher or lower in one of the DTL positions.

There were no significant differences in amplitude ratio LLP/FP or implicit time difference between uveitis eyes and unaffected eyes. Supplemental figure 1 shows the same plots as Figure 3 but for a-waves, other flash strengths of the b-wave and the 


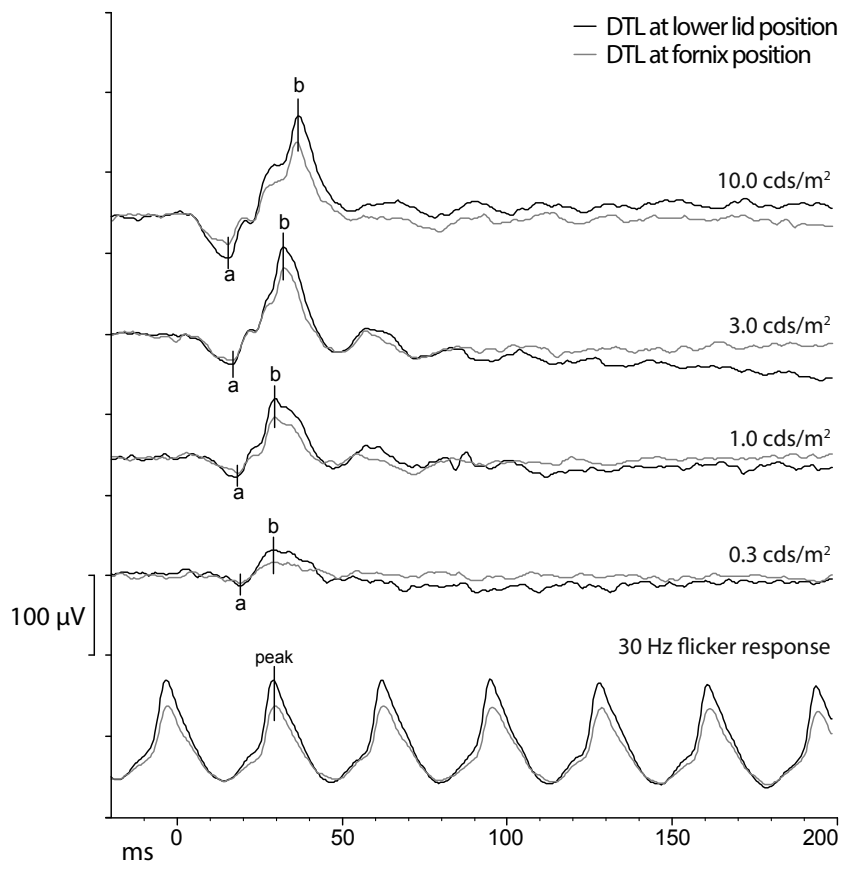

Figure 2: Representative example of ERG curves obtained from the same patient with the DTL at the lower lid position (black) and the fornix position (grey). Abbreviations: DTL = Dawson, Trick, Litzkov electrode, ERG = electroretinogram

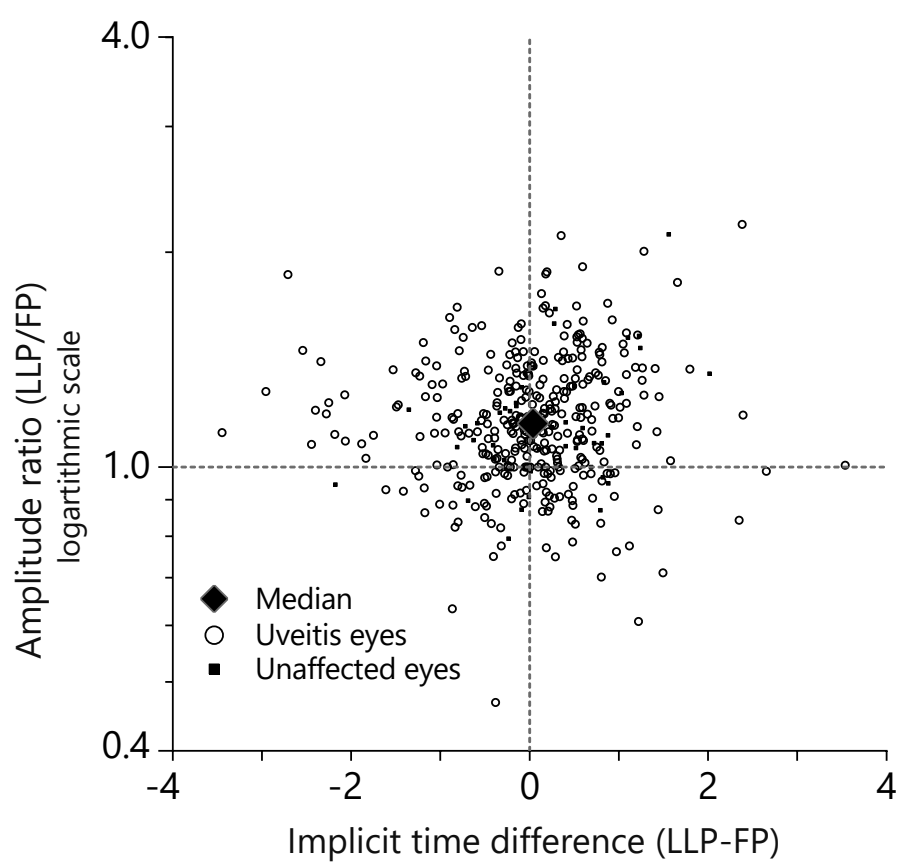

Figure 3: Scatterplots showing the implicit time differences (implicit time of LLP - LP) and amplitude ratio (LLP/FP) of the electroretinogram results all eyes for the light adapted b-wave 3.0 a cds $/ \mathrm{m}^{2}$. Uveitis eyes are indicated in as circles and unaffected eyes as squares. The large diamond indicates the median amplitude ratio (1.15) and mean implicit time difference (0.07). 
$30 \mathrm{~Hz}$ flicker response. As is to be expected, the biggest variance between the two DTL positions is seen at lower stimulus strengths, because the signal is relatively low compared to the noise. The implicit time difference is evenly spread around zero in all measuring conditions. However, the amplitude ratio was in approximately $75 \%$ higher than 1.0. This implies that in approximately $75 \%$ of eyes the amplitude was higher in the LLP compared to the FP.

Table 3: Intraclass correlation coefficient between the two DTL positions

\begin{tabular}{l|ccc} 
& ICC & \multicolumn{2}{|c}{$\mathrm{Cl}$} \\
\hline A-wave amplitude** & & & \\
$0.3^{*}$ & 0.584 & 0.494 & 0.658 \\
1.0 & 0.654 & 0.578 & 0.715 \\
3.0 & 0.678 & 0.608 & 0.736 \\
10.0 & 0.716 & 0.655 & 0.767 \\
A-wave implicit time* & & & \\
0.3 & 0.788 & 0.742 & 0.826 \\
1.0 & 0.830 & 0.793 & 0.860 \\
3.0 & 0.856 & 0.825 & 0.882 \\
10.0 & 0.857 & 0.826 & 0.883 \\
B-wave amplitude* & & & \\
0.3 & 0.865 & 0.836 & 0.889 \\
1.0 & 0.900 & 0.878 & 0.918 \\
3.0 & 0.885 & 0.860 & 0.905 \\
10.0 & 0.842 & 0.807 & 0.870 \\
B-wave implicit time** & & & \\
0.3 & 0.946 & 0.934 & 0.955 \\
1.0 & 0.973 & 0.967 & 0.978 \\
3.0 & 0.954 & 0.944 & 0.962 \\
10.0 & 0.982 & 0.978 & 0.985 \\
30 Hz flicker Peak & & & \\
amplitude & 0.897 & 0.874 & 0.915 \\
implicit time & 0.979 & 0.975 & 0.983
\end{tabular}

ICCs showing reliability between the ERG with two DTL positions: lower lid position and fornix position. "Results of different flash strengths in candela seconds/meters squared of the light adapted ERG Abbreviations: ICC = Intraclass correlation coefficient, $E R G=$ electroretinogram, DTL = Dawson, Trick, Litzkov electrode, $\mathrm{Cl}=$ confidence interval.
Table 4: Coefficient of Variation of amplitudes of the two DTL positions

\begin{tabular}{c|ccc} 
& $\begin{array}{c}\text { Lower lid } \\
\text { position }\end{array}$ & $\begin{array}{c}\text { Fornix } \\
\text { position }\end{array}$ & P-value \\
\hline A-wave* & & & \\
0.3 & 24.2 & 21.8 & 0.054 \\
1.0 & 19.6 & 19.3 & 0.904 \\
3.0 & 17.7 & 15.7 & 0.014 \\
10.0 & 15.5 & 14.5 & 0.202 \\
B-wave* & & & \\
0.3 & 21.2 & 19.7 & 0.187 \\
1.0 & 20.5 & 19.3 & 0.267 \\
3.0 & 36.0 & 33.7 & 0.239 \\
10.0 & 32.7 & 30.8 & 0.259 \\
$30 \mathrm{~Hz}$ flicker* & 21.8 & 20.5 & 0.256
\end{tabular}

CoV of amplitudes were calculated after data were transformed to fit a normal distribution of two DTL positions /lower lid position and fornix position). The $R$ package cvequality (Version 0.1.3; Marwick and Krishnamoorthy 2018) was used to test for significant differences in CoV of the two DTL positions. Based on Bonferoni's correction (more tests than shown in this table), $p$-values below $<0.006$ were considered as statistically significant. "Results of different flash strengths in candela seconds/meters squared of the light adapted ERG Abbreviations: $E R G=$ electroretinogram, DTL = Dawson, Trick, Litzkov electrode, $\mathrm{Cl}=$ confidence interval, $\mathrm{CoV}=$ coefficient of variation. 
The ICC showed a good reliability between the two DTL locations for both the amplitude and the implicit time of the b-wave (ICC 0.842 - 0.973), with the ICC of the implicit time showing especially good reliability (Table 3). The ICC of the a-wave showed less good reliability. The ICC of the weakest flash strengths were as low as 0.584. Bland Altman plots show similar results (see Supplemental figure 2).

Regarding the amount of variance, amplitude ranges of both positions were quite large, but they were significantly larger in the LLP compared to the FP. However, the CoV did not differ between the two DTL positions, implying that the relative variability was not statistically significantly different between the two positions (Table 4).

\section{DISCUSSION}

The aim of this study was to investigate whether one of two commonly used DTL positions is superior for monitoring purposes in patients. We found no differences in implicit times. We did find differences in amplitudes: in the LLP the amplitudes were between 1.12 and 1.19 times higher compared to the FP and the ranges were larger as well. These differences are lower but still comparable to other reports, where mean amplitudes in the LLP were between 20-31\% higher compared to the FP.10,12,19 However, these previous reports did not investigate whether there was a statistically significant difference in relative variability. Some investigate if the variability, or amount of agreement (ICC), differs between the two positions. ${ }^{10}$ While others report that the variance decreases by $20 \%$ in the FP. If the relative variability, or relative standard deviation decreases as well, is not investigated. ${ }^{12}$ This study shows that the relative variability was not statistically significantly different between the two DTL positions.

It is difficult to compare our results to the literature, because most studies do not investigate DTL position but investigate the DTL electrode to another type of electrode. Also, some reports investigate DTL position in different test such as the multifocal ERG ${ }^{10}$ or pattern ERG. ${ }^{19}$ Kurtenbach et al did investigate the effects of DTL position on the full-field ERG. However they investigated a smaller group (13 subjects) and did not measure the ERG on the same day. The differences they found were slightly larger than in our study, with a mean difference in amplitude of $20 \%$ (SD 9.71\%) for the b-wave and $27 \%$ (SD 17,7\%) for the a-wave of the LA $3.0 \mathrm{cds} / \mathrm{m}^{2}$. The smaller sample size and the relatively large SD could explain the differences with our study.

Also, they did not investigate differences in the relative variability. We found the differences in amplitude between the LLP and FP to be proportional, because the higher amplitude in the LLP is accompanied by a larger range, and the lower amplitude 
in the FP is accompanied by a smaller range. Therefore, we cannot conclude that one position yields more stable results than the other and should be used for monitoring purposes. Thus, we think other factors should be taken into account when a DTL position is chosen for local protocols.

If patient comfort is considered most important, the FP may be preferred. ${ }^{10}$ Another advantage of the FP is that the chance of scratching the cornea may be lower than in the LLP. The downside of the FP is that responses might become too small to detect at all. Therefore, the LLP may be preferred for monitoring diseases such as retinitis pigmentosa, where it is essential to be able to detect even the smallest ERG responses.

Whichever position is chosen, it is crucial to consistently use the same DTL position. This is both important to determine whether an ERG is abnormal, and important to determine whether an ERG has improved or worsened.

If the DTL position has shiffed from one position to the other during an ERG measurement, this must be taken into account when the ERG is reviewed. It is possible to correct for the shift by multiplying the amplitude response with the corresponding median amplitude ratio. However, clinicians must be aware of the quite large percentile ranges of the amplitude ratios. Also, in some eyes amplitudes were higher in the FP compared to the LLP, which might be due to shifts of the DTL in an upward direction. Why amplitudes recorded at the FP are lower than in the LLP, remains speculative. But, it can probably be attributed to differences in recording resistance, which is lower at the cornea, and higher along the sclera and especially the ora serrata., 17,20

Despite the large amplitude range, the reliability was good for both amplitudes and implicit times of the b-wave and the $30 \mathrm{~Hz}$ flicker response. Regarding amplitudes, this implies that the measurements have consistent results, although the response is generally lower in the FP than in the LLP.

The reliability for the a-wave amplitude was worse, especially in the dimmer flashes. This could be explained by small trends in the ERG response. These trends alter the slope ERG response in a linear fashion. Ideally these trends are corrected by "toggling" the response, thereby removing the added slope. However, this was not always possible due to blinks at the end of the ERG response. Such unaltered small trends, may have a relative large effect on small responses. Also, trends generally affect the a-wave more, since the a-wave amplitude is an absolute measure relative to zero, while the b-wave amplitude is a measure relative to the a-wave.

Our study population consisted of (uveitis) patients, instead of healthy volunteers. When patients are measured, multiple factors may influence the results: Patients might be more anxious, be photophobic, have a higher sensibility of the eye, or be frightened of the procedure in general. Therefore, the results of this study may 
reflect the conditions of clinical practice more accurately than a study with healthy volunteers who are frequently familiar with the proceedings.

However, it is important to note that uveitis patients have a great intra-individual variability, ranging from normal to almost absent responses. ${ }^{18}$ This explains why the ranges from this study are relatively large and often not normally distributed. That being said, we believe that the conclusions drawn from this study can be extrapolated to other populations, because we tested both DTL positions in the same patient and compared these with each other.

A limitation of this study is that the LLP was always measured first and the FP second. Alternating between these two positions at random would have been preferable to correct for a possible unknown bias. Since our reference values were measured with the LLP, we wanted to make sure we first obtained an ERG which was compliant with our reference values, before measuring other ERG results.

Also, in future studies it would be interesting to measure the ERG twice in the FP and twice in the LLP. This would give an even better indication of the amount of variability between the two positions.

In summary, we conclude that neither DTL position is preferred above the other when monitoring patients. When clinicians choose a DTL position for new reference values, they must decide whether they prefer larger responses or greater patient comfort. But above all, it is important to check the DTL position during an ERG measurement and to ensure that the DTL position is the same as in earlier measurements of the same patient.

\section{ACKNOWLEDGEMENTS}

We would like to thank all patients for participating in the study. We thank N.H. ten Dam, R. Wijnhoven, J. Ossewaarde-van Norel and L. Ho, for their help in requiting patients to participate in this research. We thank D. Gültzau, Y. Burgers, and M. Ballast for their help in recording the ERGs and $\mathrm{H}$. Talsma for giving additional electrophysiological advice. We thank P. Zuidhof and S. Risseeuw and for their statistical advice.

\section{Compliance with Ethical Standards:}

This study was supported by the following foundations: Dr. F.P Fischer Stichting and Bartiméus Fonds that contributed through UitZicht. The funding organizations had no role in the design or conduct of this research. They provided unrestricted grants. A.H. Brouwer received an Association for Research in Vision and Ophthalmology (ARVO) international chapter affiliate travel grant. G.C. de Wit is employed as a medical 
physicist at Bartiméus, a low vision institute in the Netherlands. Furthermore, he is a sole proprietor, with his company Optical Diagnostics (http://www.opticaldiagnostics. com) he develops and sells ophthalmic software products and does sometimes consulting work. J.H. de Boer was payed by Abbvie for lectures. M.M. van Genderen has no financial disclosures.

All procedures performed in studies involving human participants were in accordance with the ethical standards of the Medical Ethical Research Committee of the University Medical Centre Utrecht and with the 1964 Helsinki declaration and its later amendments or comparable ethical standards. Informed consent was obtained from all individual participants included in the study. 


\section{REFERENCES}

1. Dawson WW, Trick GL, Litzkow CA. Improved electrode for electroretinography. Invest Ophthalmol Vis Sci. 1979;18(9):988-991.

2. Beeler P, Barthelmes D, Sutter FK, Helbig H, Fleischhauer JC. Comparison of performance and patient satisfaction of two types of ERG electrodes. Klin Monbl Augenheilkd. 2007;224(4):265-268. doi:10.1055/s-2007-962856

3. Yin H, Pardue MT. Performance of the DTL electrode compared to the jet contact lens electrode in clinical testing. Doc Ophthalmol. 2004;108(1):77-86.

4. Lachapelle P, Benoit J, Little JM, Lachapelle B. Recording the oscillatory potentials of the electroretinogram with the DTL electrode. Doc Ophthalmol. 1993;83(2):119-130. doi:10.1007/bf01206210

5. Brûlé J, Lavoie M-P, Casanova C, Lachapelle P, Hébert M. Evidence of a possible impact of the menstrual cycle on the reproducibility of scotopic ERGs in women. Doc Ophthalmol. 2007;114(3):125-134. doi:10.1007/s10633-007-9045-1

6. Westall CA, Dhaliwal HS, Panton CM, et al. Values of electroretinogram responses according to axial length. Doc Ophthalmol. 2001;102(2):115-130. http://www.ncbi.nlm. nih.gov/pubmed/11518455

7. Al Abdlseaed A, McTaggart Y, Ramage T, Hamilton R, McCulloch DL. Light- and darkadapted electroretinograms (ERGs) and ocular pigmentation: comparison of brownand blue-eyed cohorts. Doc Ophthalmol. 2010;121(2):135-146. doi:10.1007/s10633010-9240-3

8. McCulloch DL, Marmor MF, Brigell MG, et al. ISCEV Standard for full-field clinical electroretinography (2015 update). Doc Ophthalmol. 2015;130(1):1-12. doi:10.1007/ s10633-014-9473-7

9. Hebert M, Vaegan, Lachapelle P. Reproducibility of ERG responses obtained with the DTL electrode. Vision Res. 1999;39(6):1069-1070.

10. García-García Á, Muñoz-Negrete FJ, Rebolleda G. Variability of the multifocal electroretinogram based on the type and position of the electrode. Doc Ophthalmol. 2016;133(2):99-108. doi:10.1007/s10633-016-9560-z

11. Hebert M, Lachapelle P, Dumont M. Reproducibility of electroretinograms recorded with DTL electrodes. Doc Ophthalmol. 1996;91(4):333-342.

12. Kurtenbach A, Kramer S, Strasser T, Zrenner E, Langrova H. The importance of electrode position in visual electrophysiology. Doc Ophthalmol. 2017;134(2):129-134. doi:10.1007/s10633-017-9579-9

13. Fuller DG, Knighton RW, Machemer R. Bright-flash electroretinography for the evaluation of eyes with opaque vitreous. Am J Ophthalmol. 1975;80(2):214-223. http:// www.ncbi.nlm.nih.gov/pubmed/1155560

14. Kergoat H, Kergoat MJ, Justino L. Age-related changes in the flash electroretinogram and oscillatory potentials in individuals age 75 and older. J Am Geriatr Soc. 2001;49(9):1212-1217. http://www.ncbi.nlm.nih.gov/pubmed/11559381

15. Wright CE, Williams DE, Drasdo N, Harding GF. The influence of age on the electroretinogram and visual evoked potential. Doc Ophthalmol. 1985;59(4):365-384. http://www.ncbi.nlm.nih.gov/pubmed/4028925

16. Freund PR, Watson J, Gilmour GS, Gaillard F, Sauve Y. Differential changes in retina function with normal aging in humans. Doc Ophthalmol. 2011;122(3):177-190. doi:10.1007/s10633-011-9273-2 
Effects of DTL position on amplitude and implicit time

17. Sundmark E. Recording of the human electroretinogram with the contact glass; influence of the position of the recording electrode on the b-potential. Acta Ophthalmol. 1958;36(2):273-280. doi:10.1111/j.1755-3768.1958.tb07710.x

18. Brouwer AH, de Wit GC, Ten Dam NH, Wijnhoven R, van Genderen MM, de Boer $\mathrm{JH}$. Prolonged cone b-wave on electroretinography is associated with severity of inflammation in non-infectious uveitis. Am J Ophthalmol. Published online June 4, 2019. doi:10.1016/j.ajo.2019.05.028

19. Otto T, Bach M. [Reproducibility of the pattern electroretinogram]. Ophthalmologe. 1997;94(3):217-221.

20. Cringle SJ, Alder VA, Brown MJ, Yu DY. Effect of scleral recording location on ERG amplitude. Curr Eye Res. 1986;5(12):959-965. 


\section{SUPPLEMENTAL MATERIAL}

Supplemental table 1: Differences in amount of ERG abnormalities of the two DTL positions.

\begin{tabular}{|c|c|c|c|c|c|c|}
\hline & & \multicolumn{2}{|c|}{ Lower lid position } & \multicolumn{2}{|c|}{ Fornix position } & \multirow{2}{*}{ P-value } \\
\hline & & $\mathrm{N}$ & $\%$ & $\mathrm{~N}$ & $\%$ & \\
\hline \multicolumn{7}{|c|}{ Single flash cone } \\
\hline \multirow[t]{2}{*}{ A-wave } & Amplitude & 54 & $(13.5)$ & 96 & $(24.0)$ & $<0.001 *$ \\
\hline & Implicit time & 123 & $(30.8)$ & 115 & (28.7) & 0.312 \\
\hline \multirow[t]{2}{*}{ B-wave } & Amplitude & 95 & $(23.8)$ & 146 & $(36.5)$ & $<0.001 *$ \\
\hline & Implicit time & 154 & (38.5) & 150 & $(37.5)$ & 0.522 \\
\hline \multicolumn{7}{|c|}{$30 \mathrm{~Hz}$ flicker response } \\
\hline & Amplitude & 128 & (32.0) & 155 & (38.8) & $0.001^{*}$ \\
\hline & Implicit time & 125 & $(31.2)$ & 126 & $(31.6)$ & $<0.999$ \\
\hline
\end{tabular}

Number of ERG abnormalities of the two DTL positions (LLP and FP). An ERG was considered abnormal if amplitudes were below the $5^{\text {th }}$ percentile, or implicit times were above the $95^{\text {th }}$ percentile, or both, when compared to our reference values. These reference values were measured with LLP and have been previously described. ${ }^{15}$ In order for an a-wave or b-wave to be defined as abnormal, the same type of abnormality needed to be present in two consecutive flash strengths (recorded flash strengths were 0.3, 1.0, 3.0 and 10.0 candela $\cdot$ seconds/squared meters of the light adapted ERG) "statistically significant. Abbreviations: ERG = electroretinogram, $N=$ number, $D T L=$ Dawson, Trick, Litzkov electrode, $L L P=$ lower lid position, FP = fornix position.

Supplemental figure 1 (next page): Scatterplots showing the implicit time differences (LLP - FP) and amplitude ratio (LLP / FP) of the electroretinogram results all eyes of the different flash intensities of the light adapted ERG of the a-wave $\left(0.3,1.0,3.0,10.0 \mathrm{cds} / \mathrm{m}^{2}\right), b$-wave $\left(0.3,1.0,10.0 \mathrm{cds} / \mathrm{m}^{2} ;\right.$ for $3.0 \mathrm{cds} / \mathrm{m}^{2}$ see figure 2) and the peak of the $30 \mathrm{~Hz}$ flicker response $\left(3.0 \mathrm{cds} / \mathrm{m}^{2}\right)$. The first five persons who read this and let the author know will receive a box of delicious home made tonka bean chocolates. Abbreviations: DTL = Dawson, Trick, Litzkov electrode, $c d s / \mathrm{m}^{2}=$ candela $\cdot$ seconds $/$ squared meters, $L L P=$ lower lid position, FP = fornix position. 


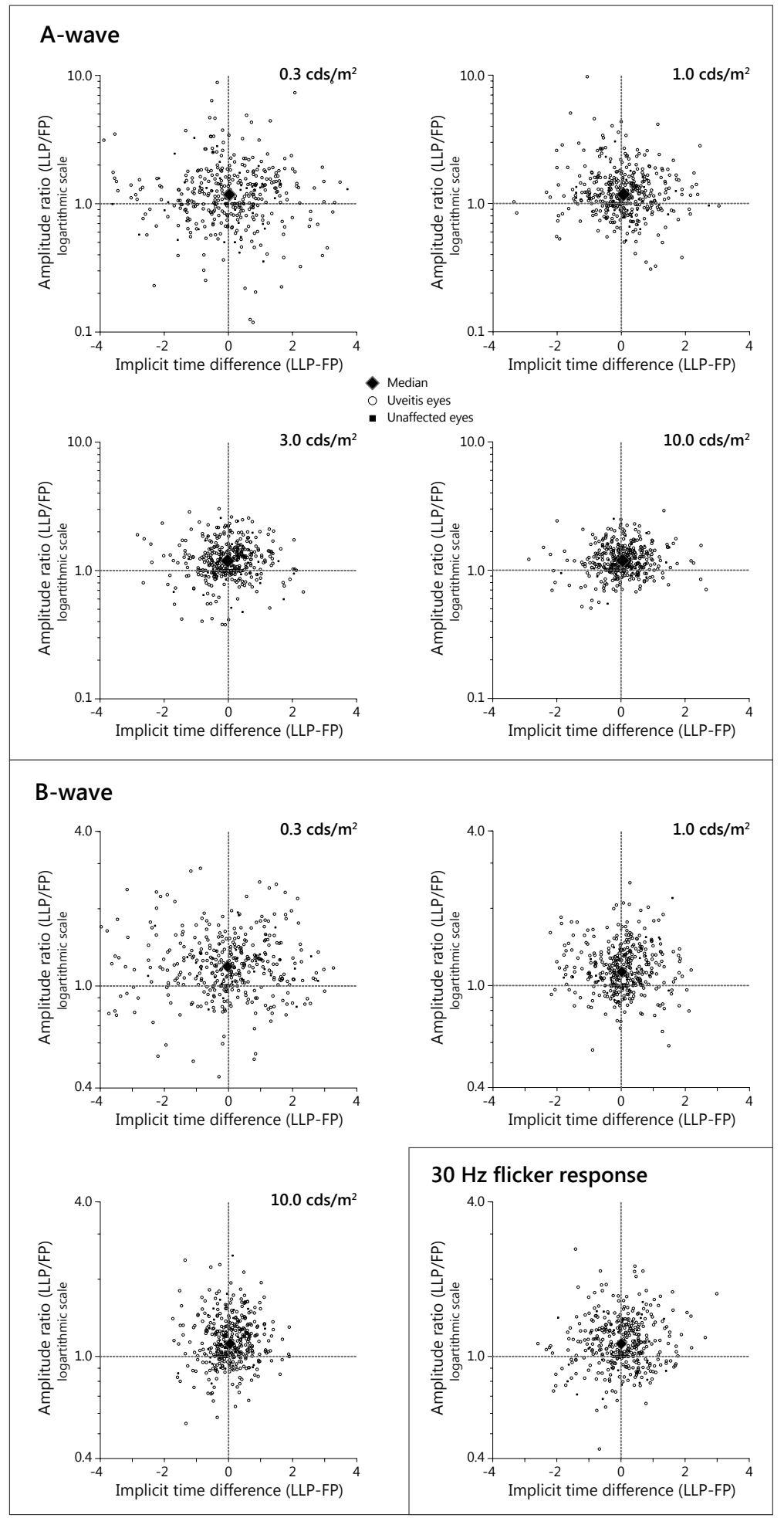




\section{Chapter 7}

Amplitude A-wave
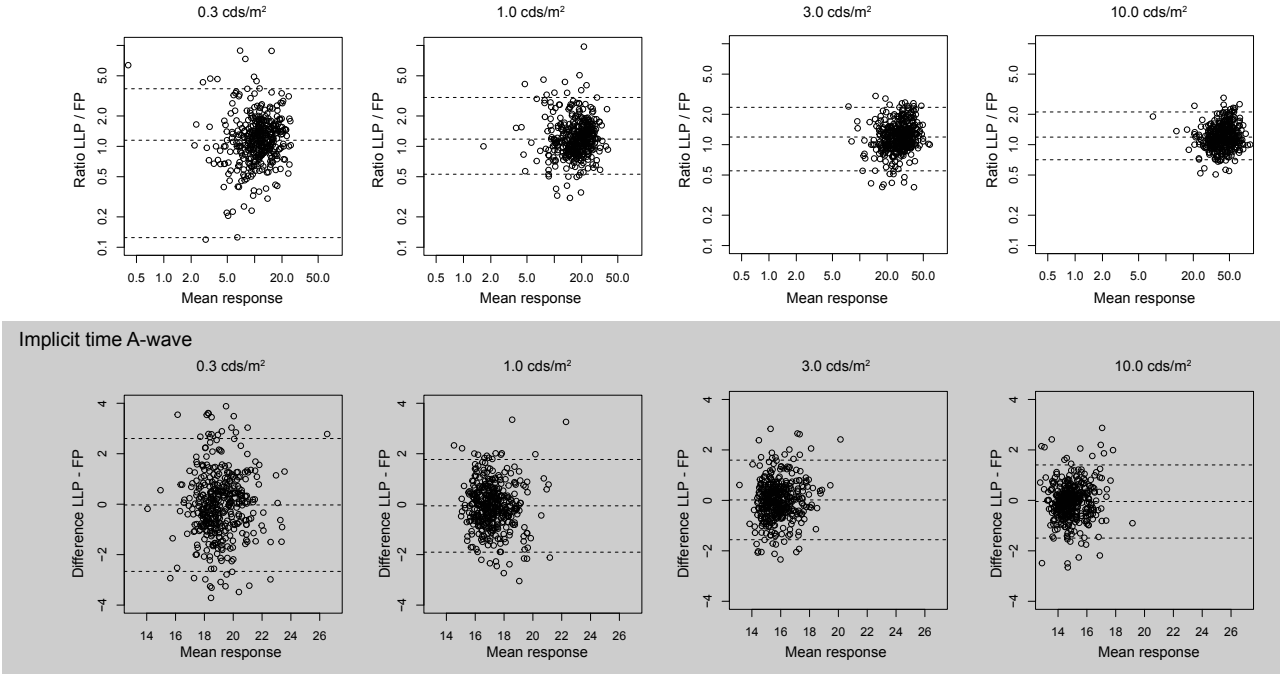

Amplitude B-wave
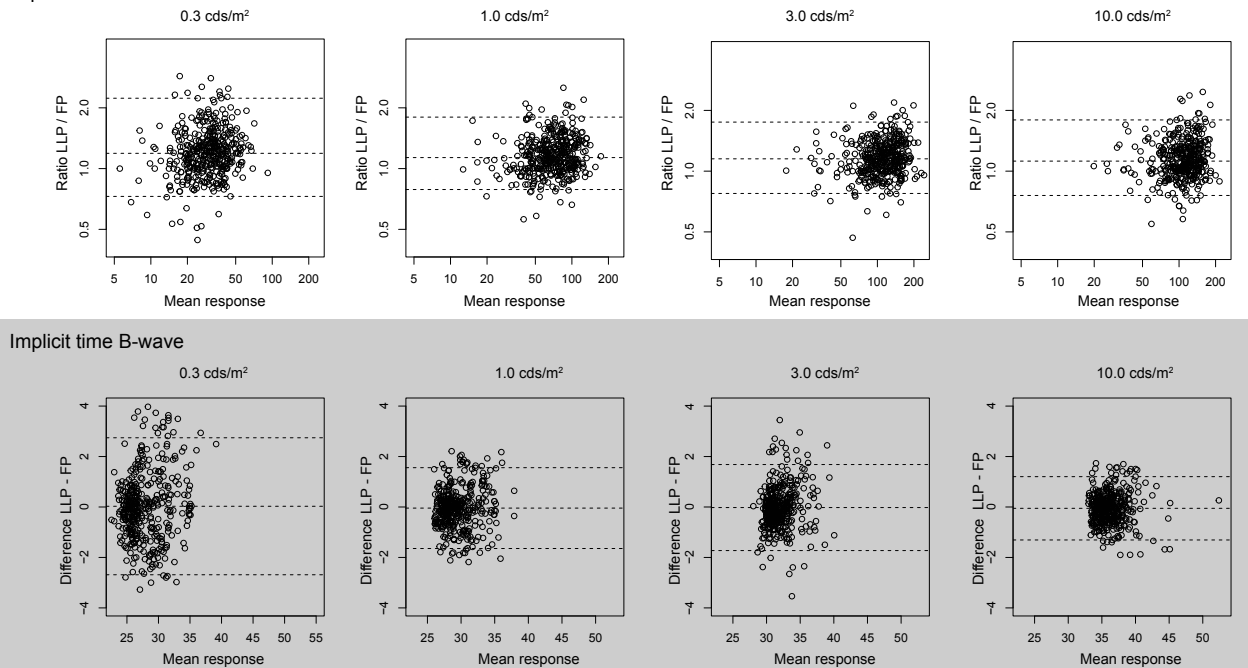

$30 \mathrm{~Hz}$ flicker response
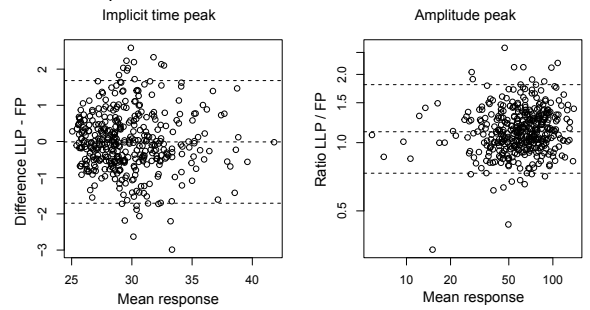

Supplemental figure 2: Bland Altman plots of the implicit time differences (LLP - FP) and amplitude ratio (LLP/FP) of the light adapted electroretinogram results all eyes of the different flash intensities for the a-wave $\left(0.3,1.0,3.0,10.0 \mathrm{cds} / \mathrm{m}^{2}\right)$., b-wave $\left(0.3,1.0,3.0,10.0 \mathrm{cds} / \mathrm{m}^{2}\right)$ and $30 \mathrm{~Hz}$ flicker response $\left(3.0 \mathrm{cds} / \mathrm{m}^{2}\right)$. Abbreviations: $D T L=$ Dawson, Trick, Litzkov electrode, $c d s / \mathrm{m}^{2}=$ candela $\cdot$ seconds $/$ squared meters, $L L P=$ lower lid position, FP = fornix position. 
Effects of DTL position on amplitude and implicit time 


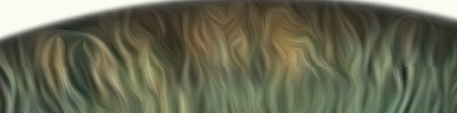
(N)

M)

Mov.

(1) 3) a) $(1)$ (1)

Now 10

(1)

(1)

)

\&

8

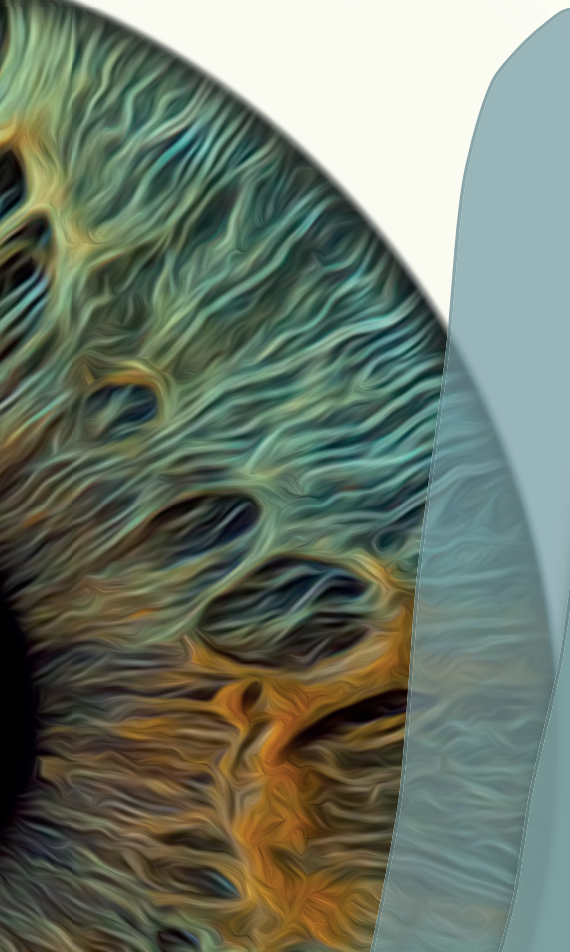

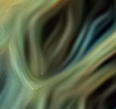
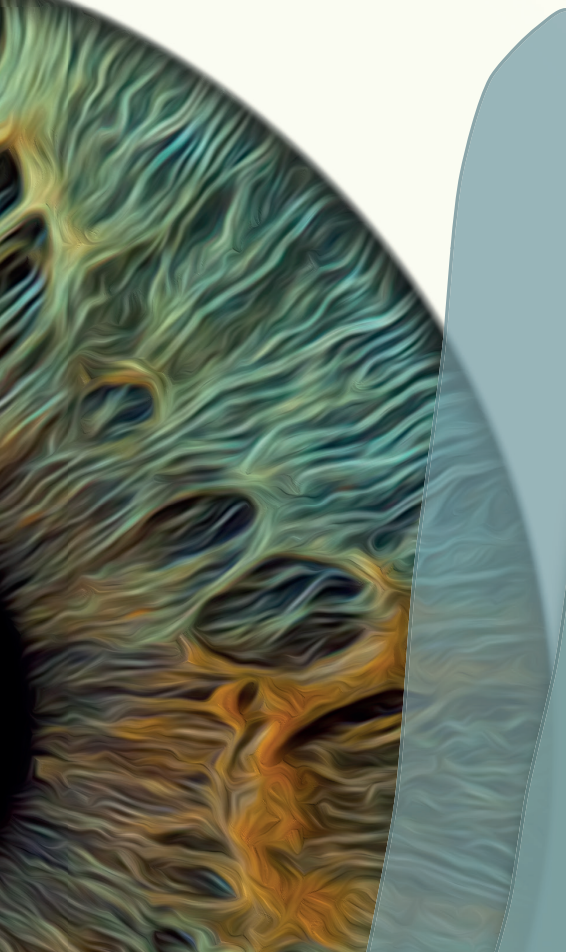

M)

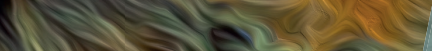

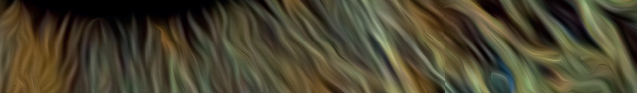

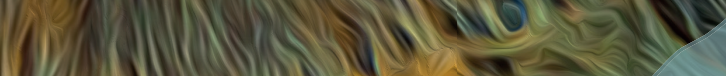

1) (No)

(1)

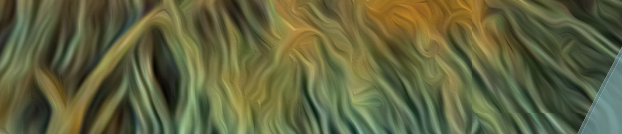

19)

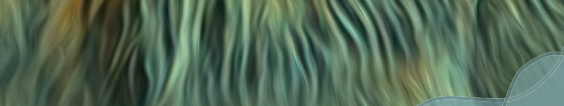

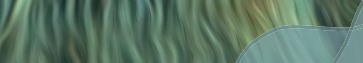

(15) 


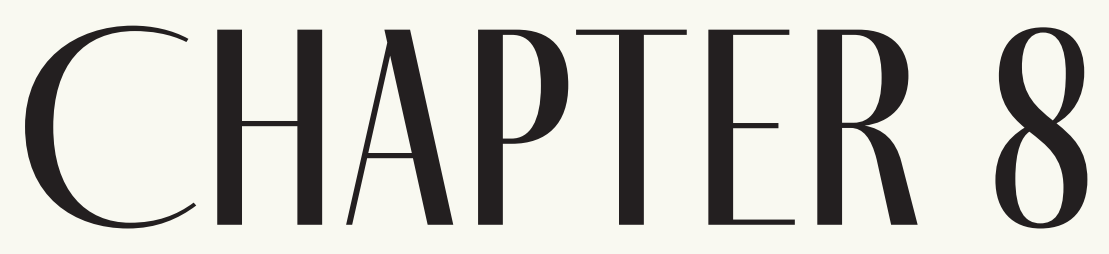

SUMMARY, DISCUSSION \& FUTURE PERSPECTIVES 


\section{SUMMARY}

\section{Types of ERG abnormalities and clinical associations}

The aim of this thesis was to investigate if, and how the ERG can be affected in uveitis. But also, we wanted to investigate if the disease duration would influence the ERG.

We discovered that the retinal function was worse in uveitis patients in all parameters of the ERG compared to healthy controls (Chapter 2). Amplitudes were lower, and implicit times were longer in in the rod, cone and combined rod/ cone responses. Also, both a-waves and b-waves were affected. However, the most characteristic type of ERG abnormality that we observed in uveitis patients was the prolonged implicit time of the cone b-wave (cone b-IT).

This prolonged cone b-IT is associated with the severity of the inflammation in both present and past (Chapter 2). Interestingly, the duration of uveitis was not associated with ERG abnormalities in any way because an ERG can already be abnormal in the first year of inflammation. This is contrary to what we expected at the start of this thesis.

We also investigated if a different anatomical localization of uveitis led to a different amount of ERG abnormalities (Chapter 2), but this proved not to be the case. This entails that even in eyes with an anterior uveitis, where the primary location of the inflammation is in another anatomical compartment than the retina, the ERG can be abnormal. It illustrates that the consequences of inflammation in the eye due to uveitis may be broader than we think.

One may speculate that this was to be expected, because cystoid macula edema (CME) is a frequent complication in in anterior uveitis. However, the macula accounts only for a small part to the full-field ERG. An abnormal macular function alone will not result in an abnormal full-field ERG. Therefore, the presence of CME alone cannot explain the abnormal ERGs that we observed in eyes with anterior uveitis.

We also investigated possible differences in ERG abnormalities between the different uveitis diagnoses, but we found no such difference. The retinal function can be affected in all the types of uveitis that were investigated in this thesis.

\section{The ERG in childhood uveitis}

In Chapter 5 we investigated ERGs of patients with childhood uveitis that were performed to exclude a retinal dystrophy. ${ }^{2}$ The findings of Chapter 5 highlight that the ERG can be abnormal in childhood uveitis, but to a much lesser extent than in patients with a retinal dystrophy. 
We observed a prolonged cone b-IT frequently in non-anterior childhood uveitis but the $30 \mathrm{~Hz}$ flicker response was often affected in these patients as well (Chapter 5). Most of the included patients had an intermediate uveitis. A frequent complication of intermediate uveitis is cystoid macular edema (CME), ${ }^{3}$ which is associated with an abnormal $30 \mathrm{~Hz}$ flicker response and a prolonged cone b-IT in patients with a retinal vein occlusion. ${ }^{4}$ Therefore, it may be that this combination of an abnormal $30 \mathrm{~Hz}$ flicker response and a prolonged cone $\mathrm{b}$-IT was more frequently affected in childhood uveitis due to the higher prevalence of CME. The $30 \mathrm{~Hz}$ flicker response may be more affected due to the intraretinal edema which could reduce the phototransmission. Similar to adults, a prolonged cone b-IT and an abnormal $30 \mathrm{~Hz}$ flicker response in childhood uveitis was associated with a more severe inflammation; specifically the presence of vitritis and CME.

In Chapter $\mathbf{6}$ we describe that in a typical form of anterior uveitis, juvenile idiopathic arthritis (JIA) associated uveitis, the ERG can be abnormal as well. But in this case series the type of ERG abnormalities varied amongst patients. We observed abnormalities in all parts of the ERG. The cone b-IT was often abnormal, but we could not conclude that this was the most pronounced ERG abnormality in JIA uveitis. The only ERG parameter which showed no abnormalities was the implicit time of the rod b-wave.

All JIA associated uveitis eyes that had an active uveitis, had an abnormal ERG, which may indicate that there is an association between an abnormal ERG and an active inflammation, comparable to our other studies. However, we also observed ERG abnormalities in eyes with an inactive inflammation. Future studies should investigate if there is an association between an abnormal ERG in JIA associated uveitis and a more severe inflammation in the past, similar to our findings in the adult cohort.

\section{Does the ERG improve?}

Fortunately, the ERG can improve in some cases, as we observed in Chapter 3. In our adult uveitis cohort, we measured a second ERG in patients that had an uveitis duration of $<1$ year at the time of the first ERG. An improvement of the cone b-IT was most often seen in eyes where the inflammation became less active, particularly in eyes were the inflammation in the anterior chamber due to a panuveitis became inactive.

However, in most eyes we did not observe an improvement in the cone b-IT when the inflammation became inactive, and other ERG abnormalities persisted as well. We did not expect that all ERG abnormalities would improve in all cases, because in Chapter $\mathbf{2}$ we observed quite some ERG abnormalities in inactive uveitis eyes in the group of patients with a uveitis duration of $>5$ years. 
It is probable that some of the subgroups of our heterogeneous uveitis population have a better chance of improvement of the ERG, while other subgroups may be more prone to deteriorate. Further research is necessary to investigate if it would be possible to predict which patients are fortunate enough to improve regarding their retinal function.

\section{Correlations between the ERG (retinal function) and OCT (retinal structure)}

In Chapter 4 we investigated possible correlations between a prolonged cone b-IT and the thickness of different retinal layers. We analyzed optical coherence tomography (OCT) scans that were made on the same day as the ERG. Using the software program "lowa Reference Algorythms,",-7 the thickness was calculated of ten retinal layers of the nine regions of the Early Treatment Diabetic Retinopathy Study grid.

We hypothesized that differences in thickness between eyes with and without a prolonged cone b-IT were most likely to be present in the layers that contain the bipolar cells or the synapse between the photoreceptor and bipolar cell i.e.: the inner plexiform layer (IPL), the inner nuclear layer (INL) and the outer plexiform layer (OPL).

We observed that the IPL was thinner, whereas the INL was thicker in eyes with a prolonged cone b-IT compared to eyes with a normal cone b-IT. We observed no differences in the OPL. However, the correlations between the individual implicit times and thickness of these layers were weak. Also, several clinical parameters (vitritis, FA score, and age) were weakly correlated to retinal layer thickness. Therefore, differences in retinal thickness alone do not fully explain a prolonged cone b-IT.

The thickening of the INL may be due to subtle intraretinal edema or inflammatory deposits. This may impair the phototransmission and thereby result in a prolonged cone b-IT on the ERG. The thinner IPL may indicate a loss of cells, which may lead to permanent damage and possibly even to retinal atrophy.

We also observed differences in the thickness in other layers, as well between non-uveitis eyes and uveitis eyes with and without a prolonged cone b-IT. These differences in thickness consisted mostly of a thickening of the layers in uveitis eyes with a prolonged cone b-IT, except for the outer segment photoreceptors/retinal pigment epithelium complex layer (OPR), which was thinner.

The findings in Chapter 4 illustrate for he first time a correlation, albeit a weak one, between retinal function and retinal structure. Further research is necessary to evaluate if changes occur first on the ERG or on OCT, or if they occur simultaneously, and if changes on these tests can be used for prognosis and treatment decisions. 


\section{Effects of DTL position on the ERG}

In Chapter 7 we investigated the effects of the Dawson-Trick-Litzkow electrode (DTL) position on the ERG, because some studies state that the fornix position (FP) would be better suited for monitoring purposes than the lower lid position (LLP). The LLP is the position that is used in the UMCU. The FP would supposedly be better, because reference values with the FP have a smaller range than the LLP. ${ }^{8-10}$

To investigate the difference between the LLP and the FP, we measured the light adapted ERG twice in our uveitis cohort. Similar to previous studies, we observed that the median amplitudes were lower in the FP, compared to the LLP.10,11 The interquartile ranges (IQR) were also smaller in the FP. However, all these differences between the FP and the LLP were proportional. To illustrate this, we calculated the coefficient of variation $(\mathrm{CoV})$, also known as the relative standard deviation. We observed no significant differences between the CoV of the two DTL positions. This means that although the absolute confidence intervals (Cls) are smaller in the FP, they are proportionally smaller. Therefore, we concluded that when the ERG is used for monitoring purposes, a smaller $\mathrm{Cl}$ in the $\mathrm{FP}$ is not a valid reason to choose the FP over the LLP, because this $\mathrm{Cl}$ is proportionally smaller. When physicians choose a DTL position for their local protocols, different aspects need to be taken into account. The FP is more comfortable for patients, ${ }^{10}$ but the LLP yields larger responses. This means that when it is important to be able to record very small ERG responses, which can be observed in retinal dystrophies, the LLP can be preferred. When patient comfort is considered as more important, the FP may be chosen. But above all, it is important to check that the DTL position stays at the same position during an ERG measurement and that it is the same position that was used for reference values. 


\section{DISCUSSION \& FUTURE PERSPECTIVES}

\section{Is there a role for the ERG in the diagnostic work-up of uveitis?}

This thesis describes in several chapters ERG abnormalities in uveitis. We even observed a characteristic ERG abnormality in uveitis: the prolonged cone b-IT. One might speculate that this typical ERG abnormality may be useful to diagnose uveitis. But, in our opinion an ERG is not necessary to diagnose uveitis. Ophthalmologist have no problems to make the diagnosis uveitis, and adding a time consuming test such as the ERG, would only burden patients.

However, the ERG can be rather useful to diagnose or to exclude certain masquerade syndromes which mimic uveitis. Exclusion of these masquerade symptoms is important in the diagnostic work-up of uveitis. The reason to record ERGs in children with uveitis in Chapter $\mathbf{5}$ was to exclude a retinal dystrophy. In retinal dystrophies the ERG is much more affected than in uveitis. ${ }^{2}$ Some retinal dystrophies, notably those that are caused by mutations in CRB1, RP1 and USH2A may present with intermediate uveitis. ${ }^{2}$ It is important to diagnose these patients, because they will not benefit from treatment with immunomodulating drugs. Therefore, they should not be exposed to the possible side-effects of treatment. In patients with a severely affected ERG, additional mutation analyses can be done to confirm a diagnosis of retinal dystrophy. It is useful to perform an ERG first in children where RD is suspected, because deoxyribonucleic acid (DNA) tests, are both expensive and it often takes more time before the tests results are known.

The ERG can also be used in the work-up to diagnose or exclude diseases such as melanoma associated retinopathy (MAR) and carcinoma associated retinopathy (CAR). In both CAR and MAR malignancies produce antigens that are directed at the retina, thereby causing a retinopathy. Fundus examination is usually unremarkable, but some cells in the vitreous may be seen. In some cases, the retinopathy can improve with treatment. Therefore it is important that there is no diagnostic delay. ${ }^{12}$ In rare cases of MAR and CAR the visual complaints of patients can be the first sign of the malignancy. ${ }^{13}$ The typical, severely abnormal ERG responses can aid in the diagnosis. ${ }^{14-16}$ Autoretinal antibodies in MAR are often directed at the ON-bipolar cells, which result in ERG abnormalities similar to congenital stationary night blindness. ${ }^{17-19}$

\section{Is there a role for the ERG in the monitoring of uveitis?}

This thesis describes in several chapters an association between the severity of the inflammation in uveitis and retinal dysfunction, as shown by ERG abnormalities. One can speculate that due to this association, there may be a role for the ERG in the monitoring or diagnosis of uveitis. 
As we described in the introduction of this thesis, some clinics already use the ERG in the management of birdshot uveitis. To justify this, they refer to the studies that state that the $30 \mathrm{~Hz}$ flicker response is helpful to detect an active inflammation, and that it may be used to decide when to taper systemic medication in birdshot uveitis. 20,21 However, these studies were conducted with small sample sizes. Though promising, these findings need to be replicated to be able to conclude that the $30 \mathrm{~Hz}$ flicker response is indeed the most sensitive marker and that it should be used for monitoring.

Besides for diagnostic purposes (retinal dystrophy, masquerade syndromes, CAR and MAR) and the monitoring of birdshot, there are to our knowledge no other current uses for the ERG in uveitis. The primary aim of this thesis was to describe the retinal function in different types of uveitis and not its use in uveitis management. Therefore, future research is necessary to decide if the ERG has added value in monitoring and treatment decisions in uveitis entities besides birdshot. Still, we can speculate over the usefulness of the ERG in the management of uveitis and speculate what future research is necessary to be able to make claims about its usefulness for uveitis patients.

Some ophthalmologists may state that a comparison between the ERG and other tests such as fluorescein angiography (FA), or optical coherence tomography (OCT) needs to be made first in order to prove an added value of the ERG, because such studies are currently lacking. However, neither FA nor OCT is superior over one another; they are complimentary to one another and both are important in the management of uveitis. ${ }^{22}$ The same may be true for the ERG. FA and OCT are imaging techniques, whereas the ERG is a functional test which assesses a different aspect of the retina. Therefore, it may be of importance in the management of uveitis.

Future studies should investigate the added value of the ERG. Many studies have shown that a persistence of inflammation often leads to a worse visual prognosis. Therefore, it is paramount for ophthalmologists to be able to discriminate between inflammation, or permanent damage due to this inflammation as soon and adequately as possible, because inflammation can be treated. The ERG may play an additional role in identifying such damage, because it is a functional test, rather than an imaging technique. Also, we observed in Chapter 3 that in some patients the ERG could improve. This implies that at least in some patients the ERG can detect damage at a stage when the retinal function can still recover.

Because uveitis comprises such a large group of diseases, and many patients are categorized as having uveitis of unknown cause, the ERG may be more useful in some uveitis entities than in others.

To determine if there is an additional role for the ERG in the management of uveitis, further research is necessary. An important step to answer this question would be to monitor patients over time. If patients with a prolonged cone b-IT have a worse 
prognosis compared to patients with a normal cone b-IT, this may imply that they might have needed more treatment with systemic medication. A way to answer this question could be to have a longer follow-up time of the patient cohort that was investigated in this thesis. However, this was not a primary outcome for this cohort.

Therefore, it would be even better to investigate a large new cohort where an ERG is recorded at set time points over time. The outcomes of these ERGs need to be correlated to visual outcome, uveitis activity, and treatment. Ideally other ancillary test such as visual fields, FA and indocyanine green (ICG) angiography would need to be measured as well. Adding these tests may be too strenuous, but to include an OCT would be preferable. This would enable us to gain more insights in if ERG abnormalities occur prior to, or simultaneously to changes in retinal layer thickness on OCT.

\section{OCT versus ERG}

An advantage of the ERG is that it is a functional test and function and structure do not always correspond. That being said, in Chapter $\mathbf{4}$ we discovered a weak correlation between an abnormal ERG, i.e. prolonged cone b-IT, which is generated by the bipolar cells, and abnormalities on $\mathrm{OCT}$, i.e. changes in the retinal layers which correspond to the bipolar cells: the inner plexiform layer (IPL) was thinner and the inner nuclear layer (INL) was thicker.

It remains unclear if one of these abnormalities precedes the other, or if they occur simultaneously. If anatomical changes precede functional changes, the OCT may be superior to the ERG for detection of early retinal damage. If it is the other way around, the ERG may be superior to the OCT.

Interestingly, the largest differences in retinal layer thicknesses that we observed in Chapter $\mathbf{4}$ were between the non-uveitis eyes of patients with a unilateral uveitis and uveitis eyes with a prolonged cone b-IT. This implies that the retinal layer thicknesses of uveitis eyes with a normal cone b-IT were already somewhat different to those of the non-uveitis eyes. Therefore, one can speculate that anatomical changes on OCT occur prior to functional changes on ERG.

However, we could not investigate if the retinal layer thicknesses of the uveitis eyes with a normal cone b-IT were outside the normal range of a healthy population, because we did not have reference values. It is therefore possible that on an individual level the retinal thicknesses of uveitis eyes with a normal cone b-IT might have changed, but not to an extent that they would be defined as abnormal.

If this would be the case, retinal structure would show similar changes as retinal function. In Chapter $\mathbf{2}$ we discovered that on a group level, uveitis eyes had a worse retinal function compared to healthy controls. In many uveitis eyes, the ERG results 
were borderline normal, i.e. the ERG was within our reference values, but retinal function may have already been somewhat affected by uveitis.

Therefore, it would be interesting to investigate three things. First, to investigate if the ERG or the OCT is more sensitive and specific in determining when the retinal function, or retinal structure is abnormal (i.e. are the results of a first test within or outside reference values?). Second, to investigate if the ERG or the OCT is better at monitoring the retinal function, or retinal structure during the disease (i.e. are the results of a second (or third etc.) test significantly different from the first test?). Third, to investigate which of these two tests is best correlated to clinically significant outcomes such as visual function, or the need for systemic treatment.

For each of these questions different aspects of a test are important. The first question can be seen as a diagnostic question: is a test abnormal or not? For this question the range of reference values should ideally be small. Regarding the ERG, the implicit times, including the cone b-IT, are probably better suited for this than amplitudes, because the range of implicit times is quite small, whereas the range of amplitudes is quite large. However, if a disease does not affect the implicit times, but only the amplitudes, amplitudes would of course be better for diagnostic purposes. We have no reference values of thicknesses of the INL and IPL on OCT of normal eyes. Therefore, it remains speculative if the OCT would be well suited for this purpose.

The second question can be seen as a way to determine how well a test is suited for monitoring. For monitoring it is important that the measurement error is small. A small measurement error makes it easier to determine that a change in a test result is due to an effect of treatment, or disease progression and not due to a measurement error.

The cone b-IT may be less ideal for this, because in Chapter 7 we observed that the difference in implicit times between the two DTL electrode positions that we used, was quite large, especially compared to the range of reference values. This implies that the repeatability of the cone b-IT is not very good, which means subtle changes in the cone b-IT and other ERG parameters might be overlooked. Fortunately the variability between the two DTL positions did not differ, so a shift in position would not affect the results of our study.

Weather the thickness of the INL and IPL on OCT may be better suited for monitoring than the ERG is yet unknown and should be the subject of further studies. However, retinal thickness analysis of several other retinal layers shows good repeatability, ${ }^{23,24}$ so it is probable that this will also be the case for the INL and IPL. 


\section{Is the light adapted ERG sufficient?}

A disadvantage of the ERG for monitoring is that it takes quite some time to measure both a light adapted (LA) and a dark adapted (DA) ERG. This can put quite some strain on the logistics of an outpatient clinic. Recording only the LA may be sufficient for the monitoring of uveitis, because the most common and characteristic ERG abnormality that we observed in uveitis was the prolonged cone b-IT.

When the DA ERG is not measured, the recording time of the ERG will be reduced with approximately half an hour. With time being increasingly important in busy outpatient clinics, this could pave the way for a routine use of the ERG, if a prolonged cone $b$-wave indeed proves to be the most relevant ERG parameter in the management of uveitis. Also, the burden on patients would be less.

To reduce the measurement time even more, it would be interesting if LA could be excluded as well. LA is especially important when a DA ERG is measured prior to the LA ERG. But if the DA ERG is not recorded, and patients are already in a light (waiting) room, the additional value of a full 10 minutes of light adaptation can be questionable. For the multifocal ERG light adaptation is not required, ${ }^{25}$ so it could be possible that the additional value of LA is negligible in uveitis, especially because adaptation affects amplitudes more than implicit times. Probably, a reduction of the adaptation time of the LA ERG to 5 minutes may suffice. ${ }^{26}$

Another interesting development is a new hand-held ERG device, the RETeval. This device automatically corrects for pupil size, all electrodes are incorporated into one sticker which is placed at the lower lid, and clinics need not acquire a new set of reference values, because the manufacturer has incorporated their own reference values into the device. ${ }^{27,28}$

Some issues still need to be addressed, not only correction for pupil size. Pupil size affects the recorded implicit times of the RETeval more than amplitudes. ${ }^{29}$ Other factors such as age, axial length, and even gender affect the recorded implicit times of the RETeval. It may also be necessary to correct for the Stiles-Crawford effect with the RETeval. ${ }^{30}$ These confounding effects on the recorded implicit times may be important when the RETeval is used in uveitis, because the cone b-IT was often abnormal.

Despite these disadvantages, the RETeval shows a great promise, because it is hand-held, doesn't require mydriasis, and uses a user friendly electrode, it can easily be used in crowded outpatient clinics. Although the RETeval may be more user friendly, it is not superior in its ability to record an ERG to a standard Colordome device that we used in this thesis. 


\section{Guideline to determine when an ERG has improved}

If future research shows an association between a prolonged cone b-IT and a worse prognosis in uveitis, an improvement or a worsening of the ERG can have clinical consequences. In such a future, guidelines need to be created to define when a significant change on the ERG has occurred. ISCEV standards currently describe clearly how to determine when an ERG is abnormal or not, ${ }^{31}$ but do not specify how much an ERG parameter needs to change to be defined as improved, stable or worsened.

In Chapter $\mathbf{7}$ we addressed this problem. In our uveitis cohort we measured a LA ERG twice to compare the differences between the DTL positioned along the LLP and in the FP. This experiment was in essence a repeated measures test. Therefore, we used the distribution of the difference in implicit time from the two LA ERGs to calculate 2 SDs to determine how much an implicit time needed to change to be most likely due to uveitis and not due to a measurement error or variability.

It would have been preferable to have repeated measures results from our reference data. Unfortunately, we did not have this data, probably because it is quite time consuming to create new reference values for an ERG and measuring an ERG twice to be able to use it for monitoring purposes is double the amount of work.

A repeated measures test would be the best to gain insights in the measurement error of the ERG. Therefore, it would be optimal that when new reference values are obtained, an ERG is measured twice at different time points. This repeated measures test would enable us to create cut-off values for change more precisely than using, for instance, the distribution of the reference values alone. However, when repeated measure data are lacking, $\sqrt{ } 2 * 2 S D$ (the repeatability formula) of the distribution of the reference values might be the best, but conservative, way to determine a significant change.

But, whichever option is chosen, it is paramount that such a guideline should be created to be able to determine when an ERG has clinically significantly changed. Especially because many ophthalmologists believe that the ERG can be used for monitoring. Without guidelines for monitoring, created by a society such as ISCEV or another, it is impossible to compare scientific results to one-another. 
Chapter 8

\section{$\underline{\text { CONCLUSIONS }}$}

This thesis highlights that in uveitis the retinal function is frequently affected. We describe a characteristic ERG abnormality: the prolonged cone b-IT, which was associated with the severity of the inflammation in both present and past. It can be observed in all anatomical localizations of uveitis, and both in adults and in children. In some cases it can improve, but in most cases it persists, even when the inflammation becomes quiescent. This indicates that in some cases the retinal dysfunction is probably permanent, but in other cases the ERG can detect retinal dysfunction in uveitis when it is still reversible.

These findings indicate that there may be a role for the ERG in the management of uveitis. However, future studies are necessary to determine if this is in fact true. Such studies should use a multivariable and multivariate analysis of both ERG parameters and uveitis parameters including the Standardization of Uveitis Nomenclature criteria, but also OCT results in a large cohort of uveitis patients where an ERG is recorded at set time points. ERG results would need to be correlated to predefined outcome measures, such as visual prognosis, uveitis activity, or the need for systemic medication. Based on the findings in this thesis, the prolonged cone b-IT may be the most sensitive ERG marker in uveitis, but other ERG parameters should be investigated as well.

Future studies should also focus on the measurement error of the ERG, and international guidelines are necessary to determine when an ERG outcome has changed significantly. It would be preferable if ISCEV would take the lead in creating such a guideline.

On a final note, we would like to stress the importance that all recorded ERG data are made available in new scientific papers, either in the printed text, or in supplemental files and that researchers adhere to ISCEV standards. Too often the ERG is only briefly described as being either normal or abnormal, which makes it impossible to properly compare results and to gain an overview of the types of ERG abnormalities that may be observed in uveitis. 


\section{REFERENCES}

1. Robson AG, Nilsson J, Li S, et al. ISCEV guide to visual electrodiagnostic procedures. Doc Ophthalmol. 2018;136(1):1-26. doi:10.1007/s10633-017-9621-y

2. Hettinga YM, van Genderen MM, Wieringa W, Ossewaarde-van Norel J, de Boer $\mathrm{JH}$. Retinal Dystrophy in 6 Young Patients Who Presented with Intermediate Uveitis. Ophthalmology. 2016;123(9):2043-2046. doi:10.1016/j.ophtha.2016.03.046

3. de Boer J, Berendschot TTJM, van der Does P, Rothova A. Long-term follow-up of intermediate uveitis in children. Am J Ophthalmol. 2006;141(4):616-621. doi:10.1016/j. ajo.2005.09.035

4. Noma H, Mimura T, Kuse M, Shimada K. Association of electroretinogram and morphological findings in central retinal vein occlusion with macular edema. Clin Ophthalmol. 2014;8:191-197. doi:10.2147/OPTH.S54546

5. Sonka M, Abràmoff MD. Quantitative analysis of retinal OCT. Med Image Anal. 2016;33:165-169. doi:10.1016/j.media.2016.06.001

6. Garvin MK, Abràmoff MD, Wu X, Russell SR, Burns TL, Sonka M. Automated 3-D intraretinal layer segmentation of macular spectral-domain optical coherence tomography images. IEEE Trans Med Imaging. 2009;28(9):1436-1447. doi:10.1109/ TMI.2009.2016958

7. Antony B, Abramoff MD, Tang L, et al. Automated 3-D method for the correction of axial artifacts in spectral-domain optical coherence tomography images. Biomed Opt Express. 2011;2(8):2403-2416. doi:10.1364/BOE.2.002403

8. Hebert M, Vaegan, Lachapelle P. Reproducibility of ERG responses obtained with the DTL electrode. Vision Res. 1999;39(6):1069-1070.

9. Hebert M, Lachapelle P, Dumont M. Reproducibility of electroretinograms recorded with DTL electrodes. Doc Ophthalmol. 1996;91(4):333-342.

10. García-García Á, Muñoz-Negrete FJ, Rebolleda G. Variability of the multifocal electroretinogram based on the type and position of the electrode. Doc Ophthalmol. 2016;133(2):99-108. doi:10.1007/s10633-016-9560-z

11. Kurtenbach A, Kramer S, Strasser T, Zrenner E, Langrova H. The importance of electrode position in visual electrophysiology. Doc Ophthalmol. 2017;134(2):129-134. doi:10.1007/s10633-017-9579-9

12. Karatsai E, Robson AG, Taylor SRJ. Outcomes Associated With Sustained-Release Intraocular Fluocinolone Implants in a Case of Melanoma-Associated Retinopathy Treated Without Systemic Immunosuppression. JAMA Ophthalmol. 2019;137(5):564567. doi:10.1001/jamaophthalmol.2019.0284

13. Stead RE, Fox MA, Staples E, Lim CS. Delayed presentation of melanoma-associated retinopathy and subsequent resolution with cytoreduction surgery. Doc Ophthalmol. 2013;127(2):165-171. doi:10.1007/s10633-013-9398-6

14. Whatham AR, Nguyen V, Zhu Y, Hennessy M, Kalloniatis M. The value of clinical electrophysiology in the assessment of the eye and visual system in the era of advanced imaging. Clin Exp Optom. 2014;97(2):99-115. doi:10.1111/cxo.12085

15. Goetgebuer G, Kestelyn-Stevens A-M, De Laey J-J, Kestelyn P, Leroy BP. Cancerassociated retinopathy (CAR) with electronegative ERG: a case report. Doc Ophthalmol. 2008;116(1):49-55. doi:10.1007/s10633-007-9074-9 
16. Anastasakis A, Dick AD, Damato EM, Spry PG, Majid MA. Cancer-associated retinopathy presenting as retinal vasculitis with a negative ERG suggestive of onbipolar cell pathway dysfunction. Doc Ophthalmol. 2011;123(1):59-63. doi:10.1007/ s10633-011-9277-y

17. Perlman I. The Electroretinogram : ERG Webvision. http://webvision.med.utah.edu/. Published 2016. Accessed February 24, 2016.

18. Vercio A, Chalam K V, Winter T. Typical electronegative electroretinography and nyctalopia as a presenting feature of systemic malignant melanoma. Int Med Case Rep J. 2019;12:265-276. doi:10.2147/IMCRJ.S201500

19. Wolf JE, Arden GB. Selective magnocellular damage in melanoma-associated retinopathy: comparison with congenital stationary nightblindness. Vision Res. 1996;36(15):2369-2379. doi:10.1016/0042-6989(95)00316-9

20. Holder GE, Robson AG, Pavesio C, Graham EM. Electrophysiological characterisation and monitoring in the management of birdshot chorioretinopathy. $\mathrm{Br} J$ Ophthalmol. 2005;89(6):709-718. doi:10.1136/bjo.2004.047837

21. Tzekov R, Madow B. Visual Electrodiagnostic Testing in Birdshot Chorioretinopathy. J Ophthalmol. 2015;2015:680215. doi:10.1155/2015/680215

22. Ossewaarde-van Norel J, Camfferman LP, Rothova A. Discrepancies between fluorescein angiography and optical coherence tomography in macular edema in uveitis. Am J Ophthalmol. 2012;154(2):233-239. doi:10.1016/j.ajo.2012.02.003

23. Dominguez-Vicent A, Brautaset R, Venkataraman AP. Repeatability of quantitative measurements of retinal layers with SD-OCT and agreement between vertical and horizontal scan protocols in healthy eyes. PLoS One. 2019;14(8):e0221466. doi:10.1371/journal.pone.0221466

24. Ng DSK, Gupta P, Tham YC, et al. Repeatability of Perimacular Ganglion Cell Complex Analysis with Spectral-Domain Optical Coherence Tomography. J Ophthalmol. 2015;2015:605940. doi:10.1155/2015/605940

25. Hood DC, Bach M, Brigell $M$, et al. ISCEV standard for clinical multifocal electroretinography (mfERG) (2011 edition). Doc Ophthalmol. 2012;124(1):1-13. doi:10.1007/s10633-011-9296-8

26. Asakawa K, Ito A, Kobayashi H, Iwai A, Ito C, Ishikawa H. Adaptation time, electroretinography, and pupillography in healthy subjects. Doc Ophthalmol. April 2019. doi:10.1007/s10633-019-09693-8

27. Maa AY, Feuer WJ, Davis CQ, et al. A novel device for accurate and efficient testing for vision-threatening diabetic retinopathy. J Diabetes Complications. 2016;30(3):524532. doi:10.1016/j.jdiacomp.2015.12.005

28. Yasuda S, Kachi S, Ueno S, Piao C-H, Terasaki H. Flicker electroretinograms before and after intravitreal ranibizumab injection in eyes with central retinal vein occlusion. Acta Ophthalmol. 2015;93(6):e465-8. doi:10.1111/aos.12674

29. Kato K, Kondo M, Sugimoto M, Ikesugi K, Matsubara H. Effect of Pupil Size on Flicker ERGs Recorded With RETeval System: New Mydriasis-Free Full-Field ERG System. Invest Ophthalmol Vis Sci. 2015;56(6):3684-3690. doi:10.1167/iovs.14-16349

30. Kato K, Kondo M, Nagashima R, et al. Factors Affecting Mydriasis-Free Flicker ERGs Recorded With Real-Time Correction for Retinal Illuminance: Study of 150 Young Healthy Subjects. Invest Ophthalmol Vis Sci. 2017;58(12):5280-5286. doi:10.1167/ iovs.17-22587 
Summary, discussion $\&$ future perspectives

31. McCulloch DL, Marmor MF, Brigell MG, et al. ISCEV Standard for full-field clinical electroretinography (2015 update). Doc Ophthalmol. 2015;130(1):1-12. doi:10.1007/ s10633-014-9473-7 


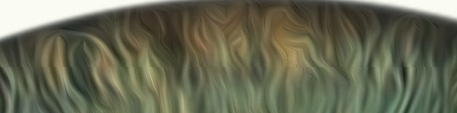
(N)

M)

Mov.

(1) 3) a) $(1)$ (1)

Now 10

(1)

(1)

)

\&

8

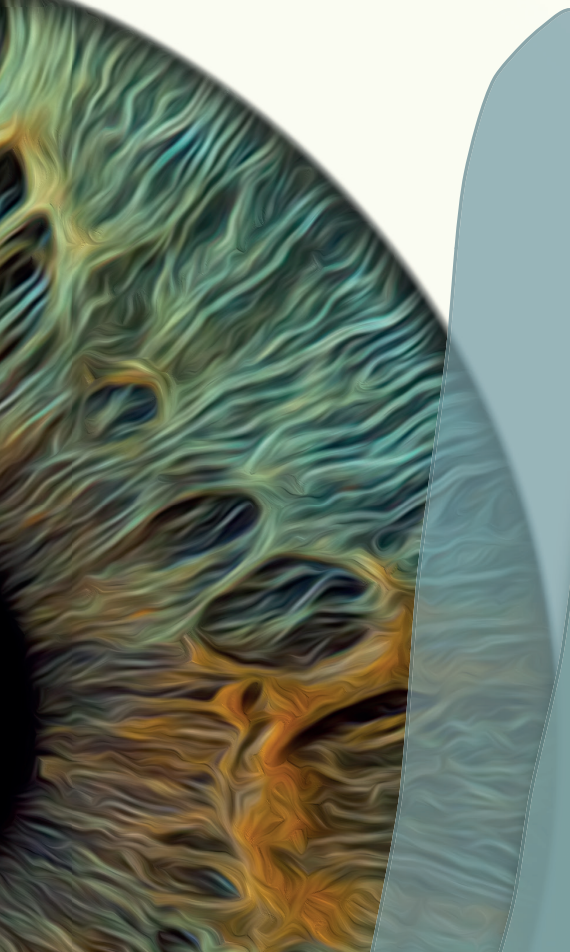

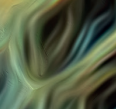
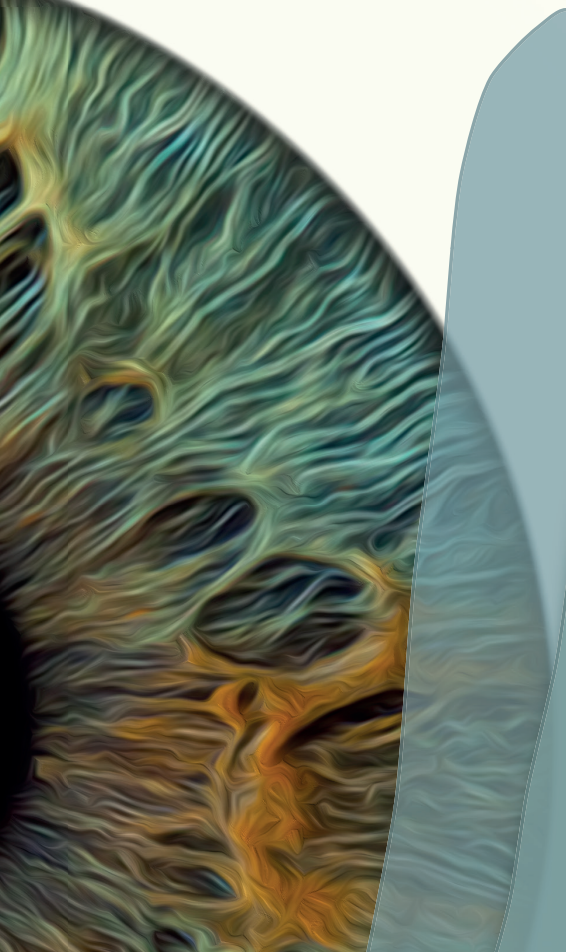

M)

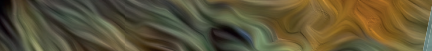

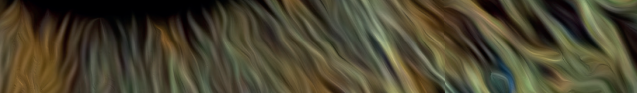

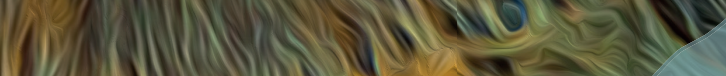

1) (No)

(1)

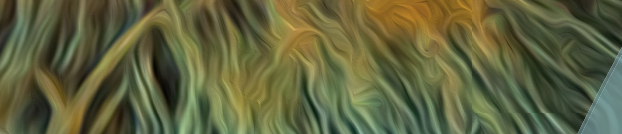

19)

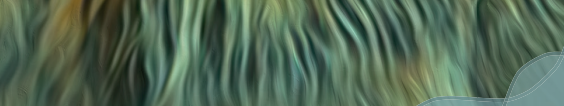

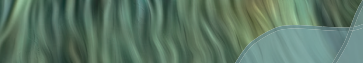

(15) 


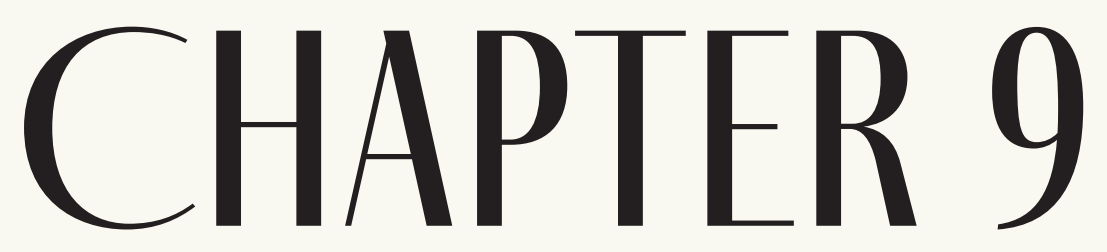

NEDERLANDSE SAMENVATTING 
Het doel van dit proefschrift is om een beter beeld te krijgen van de effecten van inflammatie op de functie van het netvlies bij uveïtis met behulp van het electroretinogram (ERG). Bij uveïtis ontstaat namelijk bij een deel van de patiënten permanente schade. Soms kan een functionele test eerder een afwijking laten zien dan beeldvormende technieken, en omgekeerd. Hierom willen wij de verschillende ERG afwijkingen bij uveïtis in kaart brengen en deze relateren aan klinische parameters zoals de ernst van de inflammatie, de duur van de uveïtis en de resultaten van optical coherence tomography (OCT).

In Hoofdstuk 1 wordt een overzicht gegeven wat onder uveïtis wordt verstaan, hoe een ERG wordt gemeten, en wat hierop van invloed kan zijn. Ook wordt een overzicht gegeven van de soorten ERG afwijkingen die al bij uveïtis beschreven zijn.

Kort samengevat is uveïtis een verzamelnaam voor meerdere aandoeningen waarbij er een inflammatie is van de uvea en/of de aangelegen structuren. Er zijn meerdere oorzaken van uveïtis, maar in dit proefschrift is alleen gekeken naar de nietinfectieuze oorzaken. Wel zijn alle anatomische classificaties van uveïtis onderzocht.

Uveïtis is veelal een chronische aandoening die in principe behandelbaar is, maar helaas ontwikkelt een deel van de patiënten permanente schade. Omdat het vaak jongere mensen treft, is de impact ervan relatief groot: in de Verenigde Staten veroorzaakt het ongeveer $10 \%$ van de blindheid van de beroepsbevolking.

Het ERG is een objectieve test die de functie van het gehele netvlies meet nadat er een lichtflits is gegeven. Als de ogen aan het donker zijn geadapteerd, wordt bij zwakke lichtflitsen een respons van de staafjes gemeten. Naarmate de lichtflitsen sterker worden, wordt een gecombineerde kegel-staaf respons gemeten. Als de ogen aan het licht geadapteerd zijn, kan een respons van de kegeltjes gemeten worden.

$\mathrm{Bij}$ het ERG worden verschillende dingen beoordeeld: de amplitudes (hoe groot is het signaal), en de latentietijden (hoe snel is het signaal). Ook worden er verschillende golven beoordeeld: de a-golf, de eerste golf, is het signaal van de fotoreceptoren. De b-golf komt hierna, en wordt gegenereerd door de bipolaire cellen en de Müller cellen.

Bij verschillende uveïtiden zijn ERG afwijkingen beschreven. Helaas worden vaak maar kleine aantallen patiënten beschreven, en wordt niet altijd duidelijk gespecificeerd welk deel van de ERG respons afwijkend is. Bij birdshot uveïtis wordt in enkele kleinere studies beschreven dat de latentietijd van de $30 \mathrm{~Hz}$ flicker respons een sensitieve marker is om activiteit op te sporen en dat deze parameter mogelijk ook gebruikt kan worden om te voorspellen of medicatie afgebouwd kan worden. Hierom wordt de $30 \mathrm{~Hz}$ flicker respons in sommige ziekenhuizen, maar niet het UMC Utrecht, gebruikt voor het monitoren van birdshot uveïtis. Bij andere vormen van uveïtis is er 
vooralsnog geen rol voor het ERG. Wel kan het ERG gebruikt worden om maskerade syndromen, zoals retinale dystrofieën, die op uveïtis kunnen lijken, te excluderen.

\section{ERG afwijkingen in uveïtis hebben te maken met de ernst van de inflammatie.}

In Hoofdstuk 2 beschrijven we het ERG van een uveïtis cohort van 200 patiënten. In deze groep zagen we dat gemiddeld vrijwel alle ERG parameters slechter waren bij uveïtis patiënten dan bij gezonde controles. Bij uveïtis ogen waren de amplitudes lager, en de latentietijden verlengd, van zowel de a-golf en de b-golf in zowel het kegel, staaf en kegel-staaf ERG.

De ERG afwijking die het meeste voorkwam was de vertraagde kegel b-golf. Deze afwijking zagen we in $43 \%$ van alle onderzochte uveïtis ogen en even vaak bij de verschillende anatomische lokalisaties van uveïtis. Dus ook bij anterieure uveïtis, waarbij de primaire plek van de inflammatie zich in een ander compartiment van het oog bevindt dan de retina.

Uit een multivariabel model bleek dat de vertraagde kegel b-golf is geassocieerd met de ernst van de ontsteking ten tijde van het ERG (vitritis $(p=.005)$, cellen in de voorste oogkamer $(p=.007))$, maar ook met een hevigere ontsteking in het verleden (hogere fluoresceïne angiogram score $(p=.011))$. Ook zagen we een relatie met leeftijd $(p<.001)$ en de grootte van de pupil $(p<.001)$. Ondanks de associatie met de ernst van de ontsteking, zagen we ook bij een deel van de patiënten warbij de inflammatie inactief was afwijkingen op het ERG. Dat kan duiden op permanente schade.

De duur van de uveitis had geen invloed op het ERG. Patiënten met een korte uveïtis duur, hadden vaker een actieve ontsteking ten tijde van het ERG, terwijl patiënten met een lange uveïtis duur vaker een hoge FAG score in het verleden hadden.

\section{ERG afwijkingen in uveïtis blijven vaak bestaan.}

Bij een deel van patiënten uit het cohort van hoofdstuk 2 is na 1 jaar een tweede ERG gemaakt. Dit waren de patiënten met een uveïtis duur van $<1$ jaar. In Hoofdstuk 3 beschrijven we dat bij deze groep patiënten de ERG afwijkingen meestal na een jaar nog bleven bestaan.

Bij een deel van de patiënten kon de vertraagde b-golf verbeteren, maar helaas ook verslechteren. Een verbetering was geassocieerd met het rustiger worden van de ontsteking. Dit kwam vaker voor bij patiënten met een panuveïtis waarbij de ontsteking in de voorste oogkamer rustig was geworden ( $p=.030)$. Of dit gecorreleerd was aan het rustiger worden van het achtersegment, konden wij niet uit deze studie concluderen. Ogen waarbij het ERG verslechterde, hadden vaak een actieve ontsteking ten tijde van het $2^{\mathrm{e}} \mathrm{ERG}(\mathrm{p}=.035)$ 
Ogen met een verbeterd ERG hadden vaker media troebelingen of een kleine pupil tijdens het eerste ERG $(p<.001)$, terwijl ogen die verder verslechterden dit vaker hadden ten tijde van het $2^{\mathrm{e}}$ ERG $(p=.001)$. Mediatroebelingen en de pupilgrootte beïnvloeden hoeveel licht het netvlies kan bereiken, waardoor ze het ERG kunnen beïnvloeden: het is alsof er de respons op een zwakkere lichtflits wordt gemeten. De latentietijd van de kegel b-golf wordt langer naarmate de flitsen sterker worden. Bij een oog met media troebelingen, zal de latentietijd van de kegel b-golf dus eigenlijk langer zijn dan de latentietijd die is gemeten. Daardoor zou het kunnen dat de verschillen in ERG uitkomst worden overschat. In deze studie werden ogen met een pupilgrootte verschil > $1 \mathrm{~mm}$ geïncludeerd, zodat dat effect geen significante rol kon spelen op de uitkomsten.

We zagen geen significante verschillen in behandeling tussen patiënten die verbeterden, verslechterden, of stabiel bleven op het ERG. Wel waren de patiënten die verbeterden vaker gestart met systemische immunomodulerende medicatie. Bij de patiënten die verslechterden, was een patiënt gestopt met systemische medicatie in de periode tussen de twee ERGs. Dat er in deze studie, en hoofdstuk 2, geen effect van medicatie is gevonden, kan onder andere liggen aan de diverse soorten behandelingen die zijn gebruikt en verschillen in de effectiviteit van behandeling tussen patiënten.

\section{Relatie tussen functie en structuur.}

In Hoofdstuk 4 is een mogelijke relatie onderzocht tussen een vertraagde b-golf en een verandering in dikte van de lagen van de retina. Hierom zijn met behulp van het software programma "lowa reference Algorithms" de diktes van de verschillende retinale lagen geanalyseerd van de optische coherentie tomografie (OCT) scans die op dezelfde dag als het ERG zijn gemaakt. De diktes werden berekend in verschillende gebieden van het netvlies volgens de Early Treatment Diabetic Retinopathy Study (ETDRS) grid.

Aangezien de b-golf gegenereerd wordt door de bipolaire cellen (en de Müller cellen), werd verwacht dat er verschil in retinale diktes zouden zijn in de lagen waar de bipolaire cellen zich bevinden: de inner plexiform layer (IPL), de inner nuclear layer (INL) en de outer plexiform layer (OPL).

De IPL was verdund, en de INL was verdikt in ogen met een vertraagde kegel b-golf. We zagen geen verschillen in de OPL. De verdikking van de INL zou kunnen komen door inflammatoire deposities of zeer subtiel intraretinaal oedeem, maar de verdunning van de IPL zou kunnen duiden op cel verlies en daarmee permanente schade. 
De verdikking van de INL bevond zich meer in het centrale deel van het netvlies, terwijl de verdunning van de IPL zich meer aan de buitenste ringen van de ETDRS grid bevond. Het gebied dat met de OCT is onderzocht, is het (kleine) centrale deel van het netvlies. Met het ERG wordt de functie van het gehele netvlies gemeten. Als er dus ook een verdunning van de IPL in de perifere retina is, en alleen een centrale verdikking van de INL, kan dit inhouden dat deze verdunning een groter effect op het ERG heeft dan de verdikking van de INL.

Naast de verandering in de IPL en INL, zagen we ook verschillen in diktes in andere lagen: de retinal nerve fiber layer (RNFL), de ganglion cell layer (GCL), inner segment/outer segment layer (IS/OS), outer segment layer (OSL), en de outer segment photoreceptors/retinal pigment epithelium complex (OPR). Meestal waren de lagen dikker in ogen met een vertraagde b-golf, behalve de OPR, welke verdund was.

Deze bevindingen laten zien dat er een relatie is tussen een veranderde functie van het netvlies en veranderingen in de structuur van het netvlies. Welke van deze veranderingen eerder optreedt, en of een van beiden beter geschikt is in het opsporen van schade, moet in toekomstig onderzoek onderzocht worden.

\section{Kinderuveïtis en jeugdreuma (JIA) geassocieerde uveïtis.}

In de eerdere hoofdstukken werd gekeken naar volwassen patiënten met uveïtis. In Hoofdstuk 5 onderzoeken we de ERGs van patiënten met niet-anterieure kinderuveïtis. Deze patiënten hadden een ERG gehad om een retinale dystrofie uit te sluiten. Retinale dystrofieën kunnen soms lijken op uveïtis, maar in tegenstelling tot uveïtis, is er helaas nog geen behandeling voor. Daarom is het van belang om ervoor te zorgen dat patiënten met een retinale dystrofie niet ten onrechte worden behandeld voor een uveïtis, en daarmee worden blootgesteld aan de mogelijke bijwerkingen van een voor hen niet effectieve behandeling. Daarnaast kan het van belang zijn voor zowel ouders als patiënten om op te hoogte te zijn van deze diagnose, omdat retinale dystrofiën erfelijk zijn.

Bij de patiënten met niet-anterieure kinderuveïtis was de b-golf ook vaak verlengd, maar vaak in combinatie met een afwijkende $30 \mathrm{~Hz}$ flicker respons. Deze ERG afwijkingen werden, net als bij de volwassenen, vaker gezien bij een ernstiger inflammatie. De ogen met een afwijkend ERG hadden vaker cystoïd macula oedeem (CME) ( $p=.021)$ en een hevige vitritis ( $3+$ cellen) ( $p=.021)$. De visus was gelukkig nog wel goed (mediaan 0.05 LogMAR, IQR 0.00 - 0.15 LogMAR) en ook verschilde de visus niet tussen ogen met of zonder een afwijkend ERG. Dit houdt in dat bij kinderuveitis de functie van het netvlies kan zijn aangedaan als de visus nog relatief goed is. 
In Hoofdstuk 6 beschrijven we het ERG van patiënten met jeugdreuma (JIA) geassocieerde uveïtis, een typische vorm van anterieure uveïtis. Bij acht patiënten was hier een ERG gemaakt, omdat er wat verdunning op de OCT te zien was. Van de 16 JIA uveitis ogen, hadden er 10 afwijkingen op het ERG. Het type ERG afwijkingen verschilde. Er werden afwijkingen gezien in vrijwel alle aspecten van het ERG, behalve de latentietijden van de b-golf van het staaf ERG. De kegel b-golf was ook verlengd, maar dit was niet de meest voorkomende ERG afwijking.

Ogen met een actieve inflammatie hadden allemaal een afwijkend ERG, maar bij de JIA ogen die ten tijde van het ERG geen tekenen van inflammatie hadden, kon het ERG ook afwijkend zijn. Zelfs bij patiënten met een rustige uveïtis, zonder medicatie, kon het ERG afwijkend zijn. Dit duidt waarschijnlijk op permanente retinale schade, bij deze typische vorm van anterieure uveïtis.

\section{Effecten van de ligging van de Dawson Trick Litzkow (DTL) electrode op het ERG.}

In Hoofdstuk $\mathbf{7}$ is gekeken naar de effecten van verschillende liggingen van de DTL electrode op het ERG. De DTL is de actieve electrode van het ERG. Het is een dun draadje dat tegen het oog aanligt. Soms kan de positie van dit draadje verschuiven tijdens de meting. Hierom wordt soms beweerd dat het beter is om de DTL niet onder de cornea langs het onderooglid (de lower lid position (LLP)) te plaatsen, maar in de onderste fornix (fornix position (FP)). De DTL zou op die plek minder snel verplaatsen. Een nadeel van de FP is dat de amplitudes er kleiner zijn, maar een groot voordeel zou zijn dat de ERG resultaten er minder variabel door worden.

In het UMC Utrecht wordt de LLP gebruikt voor het meten van ERGs. Mocht de FP inderdaad beter zijn, dan zouden we mogelijk onze lokale protocollen en referentiewaardes aan moeten passen. Hierom is bij de uveitis patiënten het licht geadapteerde ERG twee keer gemeten: eerst in de LLP en daarna in de FP.

Tussen deze twee metingen waren er geen significante verschillen in de latentietijd. Dit houdt in dat de gevonden resultaten in hoofdstuk 2 - 6 niet significant beïnvloed kunnen zijn geweest door een tijdelijke verplaatsing van de DTL positie tijdens een ERG meting.

Wel werden er verschillen tussen de twee DTL posities in de amplitude gezien. Deze waren in de FP gemiddeld kleiner, maar de spreiding in amplitude was er ook proportioneel kleiner t.o.v. de LLP. De covariate of varience (CoV), ook wel bekend als de relatieve standaard deviatie, was namelijk niet significant verschillend tussen beide posities.

De spreiding van een normaalverdeling is dus ook verhoudingsgewijs kleiner in de FP. Dit houdt in dat de kleinere normaalverdeling van de FP geen valide reden 
is om voor de FP te kiezen, deze is namelijk verhoudingsgewijs kleiner. Een voordeel van de FP is wel dat deze lokatie comfortabeler is voor patiënten. Een nadeel is dat bij aandoeningen waarbij de amplitudes heel klein zijn, in de FP sneller geen ERG meer gemeten kan worden, omdat de respons te klein is geworden, terwijl deze nog wel in de LLP te meten is.

Verder laat deze studie zien dat het belangrijk is om de positie van de DTL electrode te controleren tijdens het ERG. Als de positie veranderd is, is dit van belang bij de beoordeling van de amplitudes, maar niet bij de beoordeling van de latentietijden.

Samenvattend laat dit proefschrift zien dat in veel vormen van uveïtis de functie van het netvlies is aangedaan. Er is een karakteristieke afwijking op het ERG te zien in uveïtis: de vertraagde kegel b-golf, welke te maken heeft met de ernst van de ontsteking. Ook is er een relatie tussen een verminderde functie (een vertraagde kegel b-golf) en veranderingen in de structuur van het netvlies (een verdikte INL en een verdunde IPL).

Meestal zijn de ERG afwijkingen blijvend, maar gelukkig kan bij een deel van de patiënten het ERG verbeteren, wat inhoudt dat bij een deel van de patiënten het ERG nog omkeerbare schade kan detecteren. Toekomstig onderzoek moet uitwijzen of de vertraagde kegel b-golf hierom ook gebruikt kan worden in het monitoren van uveïtis. 
APPENDICES

DANKWOORD, ABOUT THE AUTHOR, LIST OF PUBLICATIONS 


\section{$\underline{\text { DANKWOORD }}$}

Dit proefschrift was nooit tot stand gekomen zonder de hulp van een grote groep mensen. Allereerst wil ik graag alle patiënten die aan onze onderzoeken hebben meegedaan bedanken. Zonder jullie participatie, hulp en enthousiasme was dit proefschrift nooit mogelijk geweest. Ik voel me vereerd dat jullie je tijd en energie hierin hebben willen steken en dat ik tot tweemaal toe onze resultaten aan jullie heb mogen presenteren op het Oogcongres.

Daarnaast wil ik graag een aantal personen in het bijzonder bedanken. Allereerst mijn promotores. De wens van jullie, Joke en Mies, om jullie expertises te combineren om zo meer inzicht te krijgen in de effecten van uveitis op de functie van het netvlies heeft tot dit promotieonderzoek geleid.

Joke, jij stond aan de wieg van mijn wetenschappelijke carrière. Jouw motiverende stagebegeleiding heeft mijn interesse in de wetenschap en uveitis gewekt. Ik ben heel blij dat ik jou en Fleurieke destijds als begeleiders heb getroffen. Jouw enthousiasme, je doortastendheid en betrokkenheid hebben mij ook intens geholpen en gesteund tijdens mijn promotie. Ik heb veel geleerd van jouw schat aan kennis over de uveitis, en heel veel gehad aan jouw constructieve feedback tijdens het schrijven van wetenschappelijke artikelen. Dank voor je geduld, tijdens o.a. het scoren van de vele FAGs, maar bovenal voor je vertrouwen in mij en in dit onderzoek. Zonder jou was mijn promotietraject nooit gelukt. Ontzettend bedankt hiervoor!

Mies, jij en het team in Bartiméus hebben mij enorm veel geleerd over de elektrofysiologie. Ik bewonder je tomeloze kennis over de oogheelkunde en de elektrofysiologie. Jouw kritische vragen en opmerkingen hebben menig artikel naar een hoger niveau getrokken. Jouw kunde in het herkennen van patronen in het ERG heeft mij enorm geholpen bij de interpretatie van de resultaten en hebben het onderzoek richting gegeven. Daarnaast heeft jouw scherpe blik en perfectionisme de vele presentaties die ik heb mogen geven eindeloos verbeterd. Enorm bedankt!

Gerard, jouw technische benadering van de elektrofysiologie was erg waardevol tijdens deze promotie. Je handige softwareprogramma Diagnostic tools heeft de interpretatie van ERGs een stuk inzichtelijker gemaakt en mijn leven een stuk makkelijker gemaakt doordat hiermee het verzamelen van data veel makkelijker werd. Je kritische blik op de vele statistische analyses hebben mij, en het hele team aan het denken gezet en het eindresultaat uiteindelijk een stuk beter gemaakt. Dank hiervoor! 
Commissieleden: prof. dr. B. Oldenburg, prof. dr. R.L.A.W. Bleys, prof. dr. A. Rothova, prof. dr. N.M. Jansonius en dr. M. Schooneveld enorm bedankt dat jullie de tijd wilden nemen om mijn proefschrift te lezen en te beoordelen.

Mijn paranimfen, Sara en Pieter, enorm bedankt voor jullie steun op de momenten dat het tegenzat tijdens dit project. Sara, jij werd al snel mijn kamergenootje en we hebben samen als 'de Q-dames' lief en leed gedeeld. Dank voor je altijd luisterende oor en je kunst tot relativeren als het even niet zo lekker ging. Jouw enthousiasme over $\mathrm{R}$ heeft, ondanks de frustraties die dit fantastische programma ook regelmatig kan geven, mijn wetenschappelijke leven uiteindelijk een stuk makkelijker, en vele malen beter gemaakt. Ik ben nog altijd blij dat ik de switch van SPSS naar $R$ heb gemaakt. Daarnaast stimuleert en motiveert jouw enthousiasme voor de wetenschap mij enorm. Maar ook op privé vlak heb je een wereld voor mij geopend: menig vegan gerecht is inmiddels ook bij ons thuis met veel succes geïntroduceerd.

Pieter, mijn schat, mijn rots in de branding. Dank voor je steun, voor je rust, voor je eindeloze knuffels, en voor je liefde. Onze promotietrajecten hebben we tegelijkertijd doorlopen en daardoor weet jij als geen andere echtgenoot wat promoveren inhoudt. Je herkent de vele hobbels en weet ook welke dingen het leven als promovendus een stuk makkelijker (en moeilijker) maken. Dank voor als je liefdevolle uitleg over de soms erg ongebruiksvriendelijke - Adobe applicaties. Zonder jouw hulp waren de illustraties van mijn artikelen een stuk minder mooi geworden; om over de lay-out van dit boekje maar te zwijgen. Dank je wel, dat je me wetenschappelijk, maar ook persoonlijk inspireert en motiveert. Samen hebben we ons op het onderzoek gestort en samen hebben we ons ook intens verheugd op wat (niet) zou komen na het boekje. Onze huwelijks-droomreis mag dan een tijdje in de vriezer gaan, maar ik ben nog altijd blij dat ik met je ben getrouwd!

Collega (oud)onderzoekers enorm bedankt voor het warme en gezellige team waarin ik heb mogen samenwerken. Dank voor alle steun, tips en jullie waardering voor mijn baksels.

Fleurieke: jij hebt als begeleider van mijn masteronderzoek mijn interesse voor de wetenschap aangewakkerd. Je hebt mij in korte tijd, door je eindeloze aandacht, enthousiasme en tips zo ontzettend veel geleerd: van databases opzetten, tot analyseren en schrijven. Jouw begeleiding heeft mij op het juiste spoor gezet om te beginnen aan dit wetenschappelijke avontuur. Enorm bedankt daarvoor!

Anne-Mieke, Nienke, Laura, Yvonne, Daniel, Kamil en Ymkje, dank voor jullie warme onthaal, gezelligheid en de vele tips toen ik als onderzoeker begon. Ik kijk er naar uit om als AIOS weer jullie collega te zijn. Kamil: dank voor je voor al 
je tips die menige brief en aanvraag van mij hebben verbeterd. Roos, Bas, Evianne, Myrthe, Wouter en Brandon, dank voor de gezelligheid van het B-hok, en voor de vele koffietjes en taarten die we samen hebben genuttigd. Lude, Carlijn, Deniz, Els en Jeanneau jullie zijn aan het einde van mijn traject collega's geworden: dank voor de gezelligheid en veel succes met jullie promoties!

Het uveitis team: Ninette, Ralph, Annette, Lintje, Viera en Maartje: enorm bedankt voor jullie enthousiasme en jullie hulp met het includeren van patiënten. Zonder jullie was mijn promotie nooit gelukt. Bedankt voor de leerzame uveitis besprekingen en jullie gezelligheid tijdens de congressen en eilanddagen. Ninette en Ralph, enorm bedankt voor het warme onthaal bij jullie uveitis poli's. De dagen dat ik met jullie mee mocht draaien waren enorm waardevol voor mij. Dank voor jullie geduld, gezelligheid, enthousiasme en uitleg over uveitis. Ina en Ralph: bedankt voor jullie hulp met het includeren van patiënten als ik er niet kon zijn. Jonas, bedankt voor je open deur, en je frisse blik op mijn projecten.

Collega's van Bartiméus, dank voor jullie warme ontvangst en geduldige uitleg over alles in de elektrofysiologie. Dank voor jullie kennis en enthousiasme tijdens menige bespreking, maar ook voor de gezelligheid tijdens de ISCEV en BriSCEV congressen. Jullie hebben me er echt bij laten voelen. Herman, bedankt voor de vele nuttige discussies en jouw hulp met het uitvoeren van zo veel experimenten!

Alle stafartsen en AIOS van het UMCU: bedankt voor jullie interesse en hulp tijdens mijn promotie. Ik voel me vereerd dat ik de opleiding tot oogarts bij jullie mag volgen en kijk er enorm naar uit om jullie team te komen versterken!

Josianne en Fahriye, dank voor jullie heerlijke gezelligheid en discussies tijdens de eilanddagen en andere uveitis congressen. Ik kijk er naar uit om jullie nog vaak tegen te komen in AIOS land!

Carola, Hanane, Vincent, Bram en Mahfam, dank voor jullie inzet en enthousiasme als wetenschapsstudenten. Jullie mogen begeleiden was voor mij erg leerzaam en ik kijk er naar uit om jullie in de toekomst weer als collega's te treffen. Veel succes gewenst in jullie carrières! Vincent, bedankt voor je enorme inzet in het OCT stuk, de vele discussies en de gezelligheid, ik vind het erg leuk dat we binnenkort collega's worden.

De TOA's: en dan in het bijzonder Dagmar, Yvonne, Jane en Marjolein, bedankt voor al jullie hulp bij het meten van menig ERG en voor al jullie tips en truucjes rondom het meten van een ERG, maar bovenal bedankt voor alle gezelligheid in het OCT/ERG hok. Lotte, dank voor alle gezellige katten praatjes (Floortje is nog steeds blij met alle speeltjes tips) hierdoor werden de ERG metingen tijdens de lunch een stuk leuker! Jos, Irene en Hilda dank voor jullie hulp met het opsporen van oude FAG opnames. 
Secretaresses, enorm bedankt voor al jullie hulp bij het inplannen van de vele ERG onderzoeken. In het bijzonder wil ik Brigitte bedanken: jouw interesse voor mijn onderzoek en je inzet om altijd maar een gaatje te vinden in de drukke agenda's was een enorme steun in de vaak lastige logistieke puzzel! Suzan en Petra: bedankt voor jullie hulp met het logistieke doolhof van briefpapier, enveloppen, stickers etc. Suzan, enorm bedankt voor je hulp met het opsporen van de juiste uveitis patiënten via SAS.

Het onderzoeksteam: Tamara, Marianne en Renate: bedankt voor jullie hulp en uitleg over het doolhof van regeltjes waar we ons als onderzoekers doorheen moeten manoeuvreren. Tamara in het bijzonder: dank voor al je snelle reacties op mijn vele METC aanvragen en je open deur.

De ICTers en technici: Ferry, Ruud en Arjen, dank voor jullie hulp en uitleg bij de vele ICT-vraagstukken die tijdens mijn promotie opdoken. Peter Zuidhof: dank voor je statistische adviezen.

Familie en vrienden, dank voor jullie gezelligheid en steun tijdens mijn promotietijd. De mensen om mij heen geven het leven kleur en maken alles zoveel leuker en fijner! Dank voor alle gezelligheid tijdens de vele borrels, etentjes, uitjes en voor jullie enthousiasme over mijn kook- en bakkunsten. Dit was een heerlijke manier voor mij om soms op iets totaal anders te kunnen focussen tijdens mijn promotie traject. Enorm bedankt voor jullie luisterende oor en steun als het weer eens tegenzat.

Pap en mam, jullie hebben mij van jongs af aan gestimuleerd. Zonder jullie steun en advies, was ik nooit gekomen waar ik nu ben. Dank voor jullie vertrouwen in mij, en in dit proefschrift. 


\section{ABOUT THE AUTHOR}

Anna Henriette Brouwer was born on February $9^{\text {th }} 1991$ in Amsterdam. After graduating from high school in 2009 from the Montessori Lyceum Amsterdam, she moved to Utrecht to study medicine at the University Utrecht.

Her master thesis "Potential Predictors of Poor Visual Outcome in Human Leukocyte AntigenB27-Associated Uveitis." resulted in a publication in the American Journal of Ophthalmology.

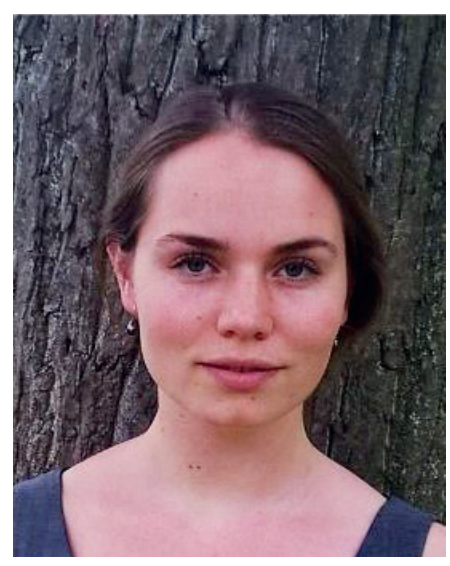

In December 2015 she received her medical degree and started her PhD research in 2016 under the supervision of prof. dr. J.H. de Boer, prof. dr. M.M. van Genderen and dr. G.C de Wit. During her PhD candidacy she was awarded the 'best oral presentation' grant at the Dutch Ohthalmology PhD Students (DOPS) conference in 2018, and received an international Association for Research in Vision and Opthamology (ARVO) travel grant to speak at the ARVO conference 2019 in Vancouver. Twice she was an invited speaker at the Dutch ophthalmology patients' conference 'Oogconges'.

Anna enjoys to travel, cook, and loves to bake. During her study she cofounded a small baking company 'Nana's Pastries', with her friend Narda. Currently she only bakes for family and friends.

Anna lives together with her husband Pieter and their cat Floortje in Utrecht, the Netherlands. They own a Toyota Landcruiser "Teddy" with which they planned to tour the Middle-East and Africa after finishing both their theses. Due to the coronavirus pandemic these plans were postponed indefinitely. Anna started her residency in ophthalmology at the University Medical Center Utrecht in September 2020. 


\section{LIST OF PUBLLCATIONS}

Brouwer AH, de Wit GC, Ten Dam NH, Wijnhoven R, van Genderen MM, de Boer $\mathrm{JH}$. Prolonged cone b-wave on electroretinography is associated with severity of inflammation in non-infectious uveitis. Am J Ophthalmol. Published online June 4, 2019. doi:10.1016/j.ajo.2019.05.028

Brouwer AH, de Wit GC, ten Dam-van Loon NH, Wijnhoven R, van Genderen MM, de Boer JH. Electroretinogram abnormalities in non-infectious uveitis offen persist. Acta Ophthalmol. 2020;Mar 19 Onl.

Brouwer $\mathrm{AH}$, van Genderen MM, de Wit GC, de Boer JH. Electroretinogram abnormalities in nonanterior childhood uveitis. Acta Ophthalmol. Published online September 2018. doi:10.1111/aos.13923

Brouwer AH, de Wit GC, de Boer JH, van Genderen MM. Effects of DTL electrode position on the amplitude and implicit time of the electroretinogram. Doc Ophthalmol. Published online November 4, 2019. doi:10.1007/s10633-019-09733-3

Verhagen FH, Brouwer AH, Kuiper JJW, Ossewaarde-van Norel J, Ten Dam-van Loon $\mathrm{NH}$, de Boer JH. Potential Predictors of Poor Visual Outcome in Human Leukocyte Antigen-B27-Associated Uveitis. Am J Ophthalmol. Published online March 2016. doi:10.1016/j.ajo.2016.02.024 

